ANDRE LESSA MANSUR

\title{
ANÁLISE DINÂMICA NÃO-LINEAR DE VIGA ESBELTA SEMI- INFINITA SOB FLEXÃO COMPOSTA COM CONTATO UNILATERAL EM APOIO ELÁSTICO: UMA APLICAÇÃO AO ESTUDO DE VIBRAÇÕES DE RISERS EM CATENÁRIA
}

Dissertação apresentada à Escola Politécnica da Universidade de São Paulo para obtenção do Título de Mestre em Engenharia Civil

São Paulo 
ANDRE LESSA MANSUR

\section{ANÁLISE DINÂMICA NÃO-LINEAR DE VIGA ESBELTA SEMI- INFINITA SOB FLEXÃO COMPOSTA COM CONTATO UNILATERAL EM APOIO ELÁSTICO: UMA APLICAÇÃO AO ESTUDO DE VIBRAÇÕES DE RISERS EM CATENÁRIA}

Dissertação apresentada à Escola Politécnica da Universidade de São Paulo para obtenção do Título de Mestre em Engenharia Civil

Área de Concentração:

Engenharia de Estruturas

Orientador: Prof. Livre-Docente

Carlos Eduardo Nigro Mazzilli

São Paulo 
Este exemplar foi revisado e alterado em relação à versão original, sob responsabilidade única do autor e com a anuência de seu orientador.

São Paulo, 10 de agosto de 2011.

Assinatura do autor

Assinatura do orientador

FICHA CATALOGRÁFICA

Mansur, André Lessa

Análise dinâmica não-linear de viga esbelta semi-infinita sob flexão composta com contato unilateral em apoio elástico: uma aplicação ao estudo de vibrações de risers em catenária / A.L. Mansur. -- ed.rev. -- São Paulo, 2011.

$142 \mathrm{p}$.

Dissertação (Mestrado) - Escola Politécnica da Universidade de São Paulo. Departamento de Engenharia de Estruturas e Geotécnica.

1. Dinâmica das estruturas 2. Vigas 3. Vibrações I. Universidade de São Paulo. Escola Politécnica. Departamento de Engenharia de Estruturas e Geotécnica II. t. 
"Não existem sons estranhos à harmonia, pois harmonia significa simultaneidade sonora. Sons estranhos à harmonia são meramente aqueles que os teóricos não foram capazes de acomodar no seu sistema de harmonia".

Harmonia, Arnold Schoenberg 


\section{Agradecimentos}

Gostaria de agradecer, primeiramente, meu orientador, Carlos Eduardo Nigro Mazzilli. A entrega e o exemplo dado na abordagem de todas as dificuldades fizeram deste trabalho mais do que uma produção científica: tornou todo o processo uma lição de vida.

Em especial, agradeço à minha noiva Fernanda Spada Villar, que ao meu lado trabalhou, dia após dia, e motivou a superação de todas as dificuldades. Sem ela, este trabalho não teria sido concluído.

Agradeço aos meus familiares, Estela, Letícia e Alfredo, pelo apoio dado nas horas de dificuldade, e pelo incentivo em todos os momentos.

Agradeço ao Prof. Celso Pesce pelo importante apoio dado no desenvolvimento deste trabalho.

Às minhas avós, Antônia Beltrame Mansur e Carolina Letícia Lessa de Castro, exemplos para a vida e espelho de força, nobreza e amor, e aos meus avôs Gonçalo Mansur e Mario Betinga de Moraes Lessa.

Agradeço aos meus queridos amigos e incentivadores Eliana e Marcelo Canal, que mostraram a busca pelo conhecimento não conhecer barreiras, limites restrições, ou pontos de chegada.

Agradeço aos Professores Antônio Ponce e Luzius Zaeslin, por terem sempre incentivado a acreditar que a ciência também é arte, e deve ser tratada e admirada como tal.

Agradeço ao Prof. Kalil Skaf, pelo que representou como incentivo no desenvolvimento deste trabalho com sua postura e conduta ímpares, e por sempre acreditar, pregar e trabalhar na melhoria constante da prática e do conhecimento da boa engenharia. 
Agradeço aos colegas Nicolau, Guilherme e Fernando Yudi, por se dedicarem e tentarem achar soluções mesmo em casos de grande dificuldade.

Agradeço à Fernanda Takafuji pelo suporte nos processamentos e obtenção de resultados.

Agradeço ao amigo Marcus Braga pelo incentivo constante e conselhos valorosos na manutenção do foco do trabalho e cumprimento das metas.

Agradeço aos colegas de trabalho e amigos, Daniel, Flávio e Jacques, pela compreensão e pelo constante incentivo na conclusão desta jornada.

Por fim, agradeço a todos aqueles, que de uma forma ou de outra, tornaram este trabalho possível. 


\section{Sumário}

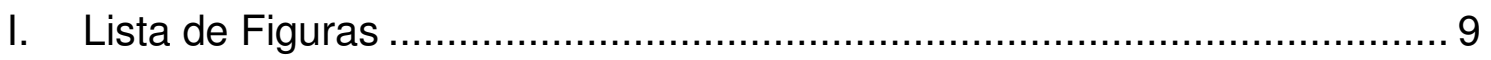

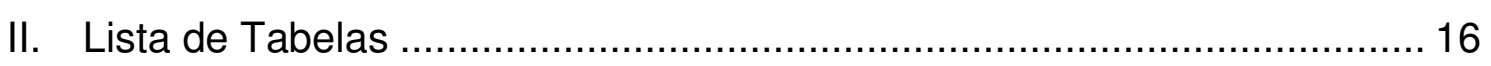

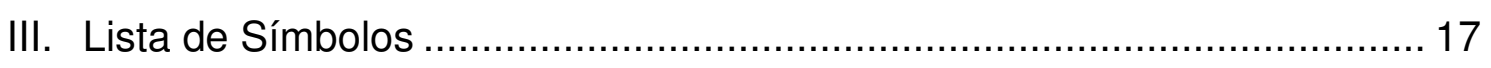

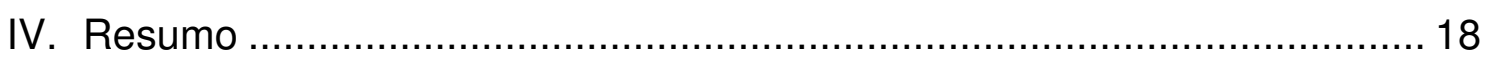

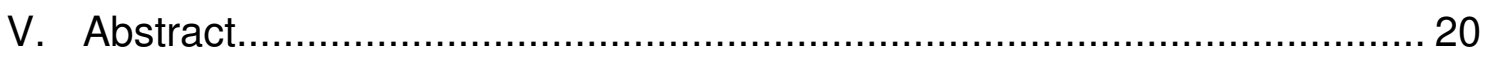

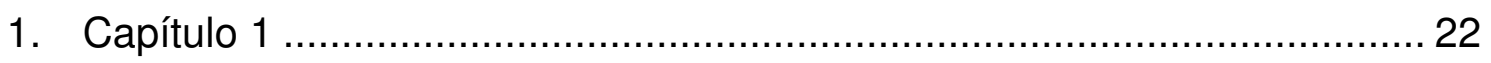

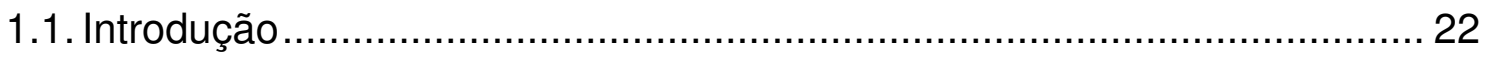

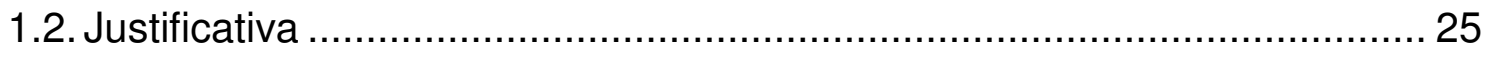

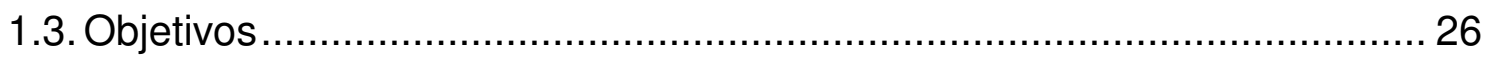

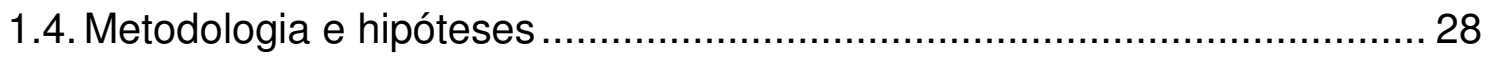

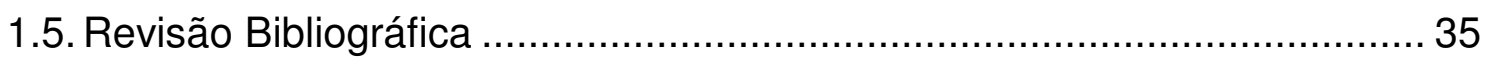

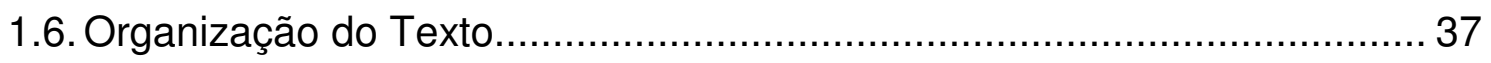

2. Capítulo 2: Modos não-lineares de vibração na flexão simples para viga esbelta semi-infinita com contato unilateral em apoio elástico ........................... 39

2.1. Formulação do problema e condições de contorno móveis........................ 39

2.2. Obtenção dos modos não lineares de vibração ......................................... 43

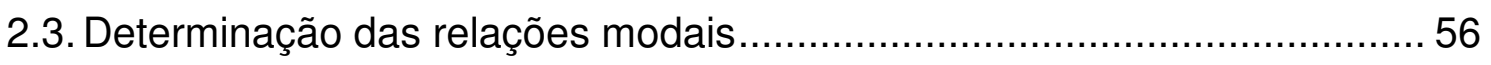

3. Capítulo 3: Modos não-lineares de vibração e modelo de ordem reduzida de viga esbelta semi-infinita com contato unilateral em apoio elástico sob flexão

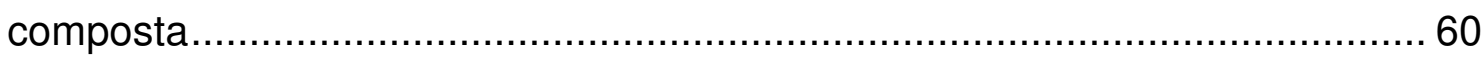

3.1. Formulação do problema com consideração do efeito de tração ............... 60

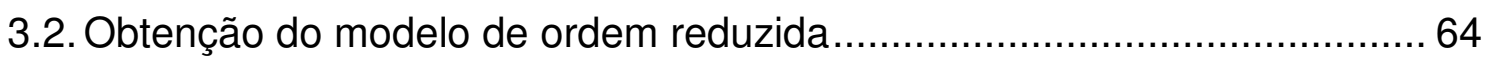

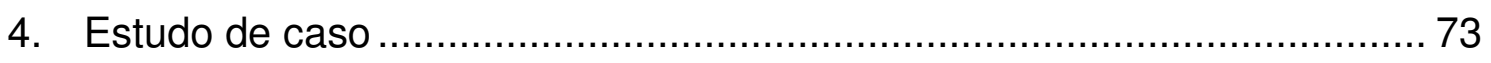

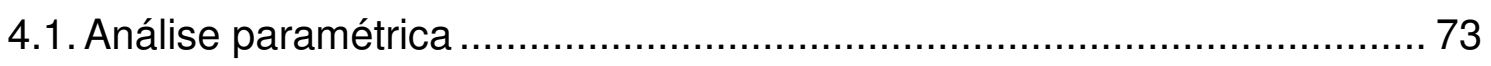

4.2. Aplicação à análise dinâmica local na região do TDP em risers flexíveis em catenária para condições de movimento imposto no topo (heave / surge) ...... 91 
4.4. Comparação dos resultados obtidos com o programa Poliflex 3D: casos sem ressonância paramétrica

4.5. Comparação dos resultados obtidos com o programa Orcaflex: casos com ressonância paramétrica

5. Conclusão e considerações finais 126

6. Referências Bibliográficas 131

7. Anexos. 135

7.1. Transformação de coordenadas para obtenção do problema com condições de contorno fixas 135

7.2. Comparação da configuração estática. 137

7.3. Parâmetro $\theta$ na consideração do efeito da tração 138

7.4. Tabela dos resultados obtidos de processamentos feitos no programa Poliflex 3D, v. 2.0.1 141

7.5. Tabela com levantamento de período de trem de ondas e altura correspondente na Bacia de Campos 


\section{Lista de Figuras}

Figura 1: Os 6 graus de liberdade de uma plataforma offshore (Fonte: Association of Marine Underwriters of San Francisco, www.amusf.com)

Figura 2: Representação esquemática dos aspectos globais do problema

Figura 3: Viga esbelta semi-infinita com contato unilateral em apoio elástico sob flexão simples.

Figura 4: Viga esbelta semi-infinita com contato unilateral em apoio elástico sob flexão composta com baixa tração

Figura 5: Viga esbelta sob flexão simples com contato unilateral em apoio elástico Figura 6: Deslocamento estático imposto na extremidade do trecho suspenso ûn

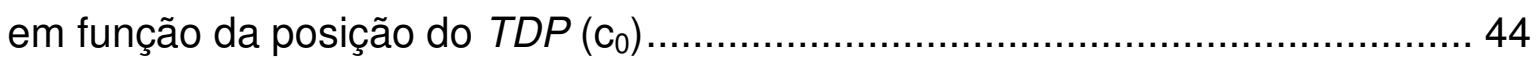

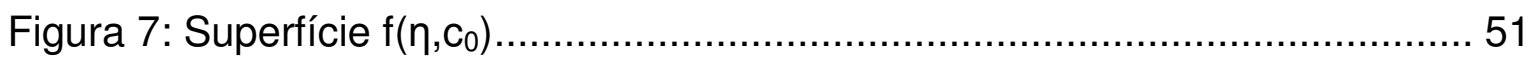

Figura 8: Uma solução (modos de vibração) correspondentes a $f(\eta)=0$ para $c_{0}=0.5$

Figura 9: Uma solução (modos de vibração) correspondentes a $f(\eta)=0$ para $c_{0}=252$ Figura 10: Duas soluções (modos de vibração) correspondentes a $f(\eta)=0$ para $\mathrm{C}_{0}=4$ 53

Figura 11: Três soluções (modos de vibração) correspondentes a $f(\eta)=0$ para $c_{0}=6$

Figura 12: Quatro soluções (modos de vibração) correspondentes a $f(\eta)=0$ para $\mathrm{C}_{0}=8$

Figura 13: Múltilplas soluções (modos de vibração) correspondentes a $f(\eta)=0$ para $\mathrm{C}_{0}=27$ 54

Figura 14: Viga esbelta semi-infinita com contato unilateral em apoio elástico sob flexo-tração estática e dinâmica 60

Figura 15: Funções modais $\zeta(\mathrm{z})$ e $\mathrm{f}(\eta)$ para $\phi=10^{2} \mathrm{~N} / \mathrm{m}^{3}, T_{0, T D P}=10 \mathrm{kN}$ e $c_{0}=3.32891$

Figura 16: Deslocamento U e velocidade V para $\phi=10^{2} \mathrm{~N} / \mathrm{m}^{3}, T_{0, T D P}=10 \mathrm{kN}$,

$\mathrm{T}_{1, T D P=15 \%} \mathrm{~T}_{0, T D P}, \xi=0 \%, \mathrm{c}_{0}=3.32891, \mathrm{~T}=17.6 \mathrm{~s}, \mathrm{U}_{0}=0.5$ e $\mathrm{V}_{0}=0.24$

Figura 17: Mapa de fase e mapa de Poincaré para $\phi=10^{2} \mathrm{~N} / \mathrm{m}^{3}, \mathrm{~T}_{0, T D P}=10 \mathrm{kN}$,

$\mathrm{T}_{1, T D P=15 \%} \mathrm{~T}_{0, T D P}, \xi=0 \%, \mathrm{c}_{0}=3.32891, \mathrm{~T}=17.6 \mathrm{~s}, \mathrm{U}_{0}=0.5$ e $\mathrm{V}_{0}=0.24$ 
Figura 18: Funções modais $\zeta(\mathrm{z})$ e $\mathrm{f}(\eta)$ para $\phi=10^{2} \mathrm{~N} / \mathrm{m}^{3}, T_{0, T D P}=10 \mathrm{kN}$ e $c_{0}=3.32891$

Figura 19: Deslocamento U e velocidade $V$ para $\phi=10^{2} \mathrm{~N} / \mathrm{m}^{3}, T_{0, T D P}=10 \mathrm{kN}$,

$\mathrm{T}_{1, T D P}=5 \% \mathrm{~T}_{0, T D P}, \xi=0 \%, \mathrm{C}_{0}=3.32891, \mathrm{~T}=17.6 \mathrm{~s}, \mathrm{U}_{0}=2$ e $\mathrm{V}_{0}=0.94$

Figura 20: Mapa de fase e mapa de Poincaré para $\phi=10^{2} \mathrm{~N} / \mathrm{m}^{3}, T_{0, T D P}=10 \mathrm{kN}$,

$\mathrm{T}_{1, T D P=5 \%} \mathrm{~T}_{0, T D P}, \xi=0 \%, \mathrm{C}_{0}=3.32891, \mathrm{~T}=17.6 \mathrm{~s}, \mathrm{U}_{0}=2$ e $\mathrm{V}_{0}=0.94$

Figura 21: Funções modais $\zeta(z)$ e $f(\eta)$ para $\phi=10^{3} \mathrm{~N} / \mathrm{m}^{3}, T_{0, T D P}=25 \mathrm{kN}$ e c $c_{0}=3.74397$

Figura 22: Deslocamento U e velocidade $V$ para $\phi=10^{3} \mathrm{~N} / \mathrm{m}^{3}, T_{0, T D P}=25 \mathrm{kN}$,

$\mathrm{T}_{1, T D P=15 \%} \mathrm{~T}_{0, T D P}, \xi=1 \%, \mathrm{C}_{0}=3.74397, \mathrm{~T}=7.1 \mathrm{~s}, \mathrm{U}_{0}=1$ e $\mathrm{V}_{0}=0.37$

Figura 23: Mapa de fase e mapa de Poincaré para $\phi=10^{3} \mathrm{~N} / \mathrm{m}^{3}, \mathrm{~T}_{0, T D P}=25 \mathrm{kN}$,

$\mathrm{T}_{1, T D P=15 \%} \mathrm{~T}_{0, T D P}, \xi=1 \%, \mathrm{C}_{0}=3.74397, \mathrm{~T}=7.1 \mathrm{~s}, \mathrm{U}_{0}=1$ e $\mathrm{V}_{0}=0.37$

Figura 24: Funções modais $\zeta(z)$ e $f(\eta)$ para $\phi=10^{3} \mathrm{~N} / \mathrm{m}^{3}, T_{0, T D P}=30 \mathrm{kN}$ e $c_{0}=3.41776$

Figura 25: Deslocamento $U$ e velocidade $V$ para $\phi=10^{3} \mathrm{~N} / \mathrm{m}^{3}, T_{0, T D P}=30 \mathrm{kN}$,

$\mathrm{T}_{1, T D P}=5 \% \mathrm{~T}_{0, T D P}, \xi=0.5 \%, \mathrm{C}_{0}=3.41776, \mathrm{~T}=5.5 \mathrm{~s}, \mathrm{U}_{0}=1$ e $\mathrm{V}_{0}=0.48$

Figura 26: Mapa de fase e mapa de Poincaré para $\phi=10^{3} \mathrm{~N} / \mathrm{m}^{3}, T_{0, T D P}=30 \mathrm{kN}$,

$\mathrm{T}_{1, T D P=5 \%} \mathrm{~T}_{0, T D P}, \xi=0.5 \%, \mathrm{C}_{0}=3.41776, \mathrm{~T}=5.5 \mathrm{~s}, \mathrm{U}_{0}=1$ e $\mathrm{V}_{0}=0.48$

Figura 27: Funções modais $\zeta(z)$ e $f(\eta)$ para $\phi=10^{4} \mathrm{~N} / \mathrm{m}^{3}, T_{0, T D P}=30 \mathrm{kN}$ e $c_{0}=5.26347$

Figura 28: Deslocamento U e velocidade V para $\phi=10^{4} / \mathrm{m}^{3}, T_{0, T D P}=40 \mathrm{kN}$,

$\mathrm{T}_{1, T D P=40 \%} \mathrm{~T}_{0, T D P}, \xi=6 \%, \mathrm{C}_{0}=5.26347, \mathrm{~T}=3.8 \mathrm{~s}, \mathrm{U}_{0}=1$ e $\mathrm{V}_{0}=0.22$

Figura 29: Mapa de fase e mapa de Poincaré para $\phi=10^{4} / \mathrm{m}^{3}, T_{0, T D P}=30 \mathrm{kN}$,

$\mathrm{T}_{1, T D P=40 \%} \mathrm{~T}_{0, T D P}, \xi=6 \%, \mathrm{C}_{0}=5.26347, \mathrm{~T}=3.8 \mathrm{~s}, \mathrm{U}_{0}=1$ e $\mathrm{V}_{0}=0.22$

Figura 30: Funções modais $\zeta(z)$ e $f(\eta)$ para $\phi=10^{4} \mathrm{~N} / \mathrm{m}^{3}, T_{0, T D P}=45 \mathrm{kN}$ e $c_{0}=4.96244$

Figura 31: Deslocamento U e velocidade V para $\phi=10^{4} \mathrm{~N} / \mathrm{m}^{3}, T_{0, T D P}=45 \mathrm{kN}$,

$\mathrm{T}_{1, T D P=15 \%} \mathrm{~T}_{0, T D P}, \xi=0.18 \%, \mathrm{C}_{0}=4.96244, \mathrm{~T}=3.3 \mathrm{~s}, \mathrm{U}_{0}=7$ e $\mathrm{V}_{0}=1.77$

Figura 32: Mapa de fase e mapa de Poincaré para $\phi=104 \mathrm{~N} / \mathrm{m}^{3}, \mathrm{~T} 0, T D P=45 \mathrm{kN}$,

$\mathrm{T} 1, \mathrm{TDP}=15 \% \mathrm{T0}, \mathrm{TDP}, \xi=0.18 \%, \mathrm{c} 0=4.96244, \mathrm{~T}=3.3 \mathrm{~s}, \mathrm{U} 0=7$ e V0=1.77. 
Figura 33: Funções modais $\zeta(z)$ e $f(\eta)$ para $\phi=10^{4} \mathrm{~N} / \mathrm{m}^{3}, T_{0, T D P}=45 \mathrm{kN}$ e $c_{0}=4.96244$

Figura 34: Deslocamento U e velocidade $V$ para $\phi=10^{4} \mathrm{~N} / \mathrm{m}^{3}, T_{0, T D P}=45 \mathrm{kN}$,

$\mathrm{T}_{1, T D P}=15 \% \mathrm{~T}_{0, T D P}, \xi=0.18 \%, \mathrm{C}_{0}=4.96244, \mathrm{~T}=3.3 \mathrm{~s}, \mathrm{U}_{0}=4$ e $\mathrm{V}_{0}=1.01$

Figura 35: Mapa de fase e mapa de Poincaré para $\phi=10^{4} \mathrm{~N} / \mathrm{m}^{3}, T_{0, T D P}=45 \mathrm{kN}$,

$\mathrm{T}_{1, T D P=15 \%} \mathrm{~T}_{0, T D P}, \xi=0.18 \%, \mathrm{c}_{0}=4.96244, \mathrm{~T}=3.3 \mathrm{~s}, \mathrm{U}_{0}=4$ e $\mathrm{V}_{0}=1.01$

Figura 36: Funções modais $\zeta(\mathrm{z})$ e f(n) para $\phi=10^{4} \mathrm{~N} / \mathrm{m}^{3}, T_{0, T D P}=30 \mathrm{kN}$ e $c_{0}=6.07773$

Figura 37: Deslocamento U e velocidade $V$ para $\phi=10^{4} \mathrm{~N} / \mathrm{m}^{3}, T_{0, T D P}=30 \mathrm{kN}$,

$\mathrm{T}_{1, T D P=6.9 \%} \mathrm{~T}_{0, T D P}, \xi=0.15 \%, \mathrm{C}_{0}=6.07773, \mathrm{~T}=4.8 \mathrm{~s}, \mathrm{U}_{0}=9.5$ e $\mathrm{V}_{0}=1.63$

Figura 38: Mapa de fase e mapa de Poincaré para $\phi=10^{4} \mathrm{~N} / \mathrm{m}^{3}, T_{0, T D P}=30 \mathrm{kN}$,

$\mathrm{T}_{1, T D P=6.9 \%} \mathrm{~T}_{0, T D P}, \xi=0.15 \%, \mathrm{C}_{0}=6.07773, \mathrm{~T}=4.8 \mathrm{~s}, \mathrm{U}_{0}=9.5$ e $\mathrm{V}_{0}=1.63$

Figura 39: Funções modais $\zeta(z)$ e $f(\eta)$ para $\phi=10^{4} \mathrm{~N} / \mathrm{m}^{3}, T_{0, T D P}=30 \mathrm{kN}$ e $c_{0}=6.07773$

Figura 40: Deslocamento U e velocidade $V$ para $\phi=10^{4} \mathrm{~N} / \mathrm{m}^{3}, T_{0, T D P}=30 \mathrm{kN}$,

$\mathrm{T}_{1, T D P}=6.9 \% \mathrm{~T}_{0, T D P}, \xi=0.15 \%, \mathrm{C}_{0}=6.07773, \mathrm{~T}=4.8 \mathrm{~s}, \mathrm{U}_{0}=9.8$ e $\mathrm{V}_{0}=1.68$

Figura 41: Mapa de fase e mapa de Poincaré para $\phi=10^{4} \mathrm{~N} / \mathrm{m}^{3}, T_{0, T D P}=30 \mathrm{kN}$,

$\mathrm{T}_{1, T D P=6.9 \%} \mathrm{~T}_{0, T D P}, \xi=0.15 \%, \mathrm{c}_{0}=6.07773, \mathrm{~T}=4.8 \mathrm{~s}, \mathrm{U}_{0}=9.8$ e $\mathrm{V}_{0}=1.68$

Figura 42: Funções modais $\zeta(z)$ e $f(\eta)$ para $\phi=10^{4} \mathrm{~N} / \mathrm{m}^{3}, T_{0, T D P}=30 \mathrm{kN}$ e c $c_{0}=6.07773$

Figura 43: Deslocamento U e velocidade $V$ para $\phi=10^{4} \mathrm{~N} / \mathrm{m}^{3}, T_{0, T D P}=30 \mathrm{kN}$,

$\mathrm{T}_{1, T D P}=6.9 \% \mathrm{~T}_{0, T D P}, \xi=0.15 \%, \mathrm{C}_{0}=6.07773, \mathrm{~T}=4.8 \mathrm{~s}, \mathrm{U}_{0}=10$ e $\mathrm{V}_{0}=1.71$ 86

Figura 44: Mapa de fase e mapa de Poincaré para $\phi=10^{4} \mathrm{~N} / \mathrm{m}^{3}, T_{0, T D P}=30 \mathrm{kN}$,

$\mathrm{T}_{1, T D P=6.9 \%} \mathrm{~T}_{0, T D P}, \xi=0.15 \%, \mathrm{C}_{0}=6.07773, \mathrm{~T}=4.8 \mathrm{~s}, \mathrm{U}_{0}=10$ e $\mathrm{V}_{0}=1.71$

Figura 45: Funções modais $\zeta(z)$ e $f(\eta)$ para $\phi=10^{4} \mathrm{~N} / \mathrm{m}^{3}, T_{0, T D P}=30 \mathrm{kN}$ e $c_{0}=6.07773$

Figura 46: Deslocamento U e velocidade $V$ para $\phi=10^{4} \mathrm{~N} / \mathrm{m}^{3}, T_{0, T D P}=30 \mathrm{kN}$,

$\mathrm{T}_{1, T D P=6.9 \%} \mathrm{~T}_{0, T D P}, \quad \xi=0.15 \%, \mathrm{C}_{0}=6.07773, \mathrm{~T}=4.8 \mathrm{~s}, \mathrm{U}_{0}=10.4$ e $\mathrm{V}_{0}=1.78$

Figura 47: Mapa de fase e mapa de Poincaré para $\phi=10^{4} \mathrm{~N} / \mathrm{m}^{3}, T_{0, T D P}=30 \mathrm{kN}$,

$\mathrm{T}_{1, T D P=6.9 \%} \mathrm{~T}_{0, T D P}, \quad \xi=0.15 \%, \mathrm{C}_{0}=6.07773, \mathrm{~T}=4.8 \mathrm{~s}, \mathrm{U}_{0}=10.4$ e $\mathrm{V}_{0}=1.78$ 
Figura 48: Mapas de fase para $\phi=10^{4} \mathrm{~N} / \mathrm{m}^{3}, \mathrm{~T}_{0, T D P}=30 \mathrm{kN}, \mathrm{T}_{1, T D P}=6.9 \% \mathrm{~T}_{0, T D P}$, $\xi=0.15 \%, c_{0}=6.07773, T=4.8 \mathrm{~s}$, para as seguintes condições iniciais (em sentido horário, a partir do quadrante superior esquerdo): $\cup_{0}=9.5$ e $V_{0}=1.63, \cup_{0}=9.8$ e $\mathrm{V}_{0}=1.68, \mathrm{U}_{0}=10$ e $\mathrm{V}_{0}=1.71$, e $\mathrm{U}_{0}=10.4$ e $\mathrm{V}_{0}=1.78$ 89

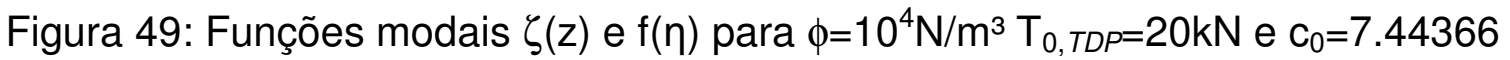

Figura 50: Deslocamento horizontal do $T D P \mathrm{x}_{\mathrm{c}}$ e velocidade $\mathrm{v}_{\mathrm{c}}$ para $\phi=10^{4} \mathrm{~N} / \mathrm{m}^{3}$, $\mathrm{T}_{0, T D P}=20 \mathrm{kN}, \xi=10 \%, \mathrm{C}_{0}=7.44366, \mathrm{~T}=10.2 \mathrm{~s}$ para $\mathrm{x}_{\mathrm{c}, 0}=0.1$ e $\mathrm{v}_{\mathrm{c}, 0}=0.03$. 95

Figura 51: Mapa de fase e mapa de Poincaré para $\phi=10^{4} \mathrm{~N} / \mathrm{m}^{3}, T_{0, T D P}=20 \mathrm{kN}$, $\xi=10 \%, c_{0}=7.44366, T=10.2 s$ para $x_{c, 0}=0.1$ e $v_{c, 0}=0.03$ 95

Figura 52: Funções modais $\zeta(z)$ e $f(\eta)$ para $\phi=10^{4} \mathrm{~N} / \mathrm{m}^{3} \mathrm{~T}_{0, T D P}=30 \mathrm{kN}$ e $\mathrm{c}_{0}=6.07773$

Figura 53: Deslocamento horizontal do $T D P \mathrm{x}_{\mathrm{c}}$ e velocidade $\mathrm{v}_{\mathrm{c}}$ para $\phi=10^{4} \mathrm{~N} / \mathrm{m}^{3}$, $\mathrm{T}_{0, T D P}=30 \mathrm{kN}, \xi=10 \%, \mathrm{c}_{0}=6.07773, \mathrm{~T}=8.1 \mathrm{~s}$ para $\mathrm{x}_{\mathrm{c}, 0}=0.1$ e $\mathrm{v}_{\mathrm{c}, 0}=0.04$. 96

Figura 54: Mapa de fase e mapa de Poincaré para $\phi=10^{4} \mathrm{~N} / \mathrm{m}^{3}, T_{0, T D P}=30 \mathrm{kN}$, $\xi=10 \%, c_{0}=6.07773, T=8.1 \mathrm{~s}$ para $\mathrm{x}_{\mathrm{c}, 0}=0.1 \mathrm{e} \mathrm{v}_{\mathrm{c}, 0}=0.04$ 96

Figura 55: Funções modais $\zeta(z)$ e f(n) para $\phi=10^{4} \mathrm{~N} / \mathrm{m}^{3} \mathrm{~T}_{0, T D P}=40 \mathrm{kN}$ e $\mathrm{c}_{0}=5.26347$

Figura 56: Deslocamento horizontal do $T D P x_{c}$ e velocidade $v_{c}$ para $\phi=10^{4} \mathrm{~N} / \mathrm{m}^{3}$, $\mathrm{T}_{0, T D P}=40 \mathrm{kN}, \xi=10 \%, \mathrm{c}_{0}=5.26347, \mathrm{~T}=4.1 \mathrm{~s}$ para $\mathrm{x}_{\mathrm{c}, 0}=0.1$ e $\mathrm{v}_{\mathrm{c}, 0}=0.08$.

Figura 57: Mapa de fase e mapa de Poincaré para $\phi=10^{4} \mathrm{~N} / \mathrm{m}^{3}, T_{0, T D P}=40 \mathrm{kN}$, $\xi=10 \%, c_{0}=5.26347, \mathrm{~T}=4.1 \mathrm{~s}$ para $\mathrm{x}_{\mathrm{c}, 0}=0.1 \mathrm{e} \mathrm{v}_{\mathrm{c}, 0}=0.08$.

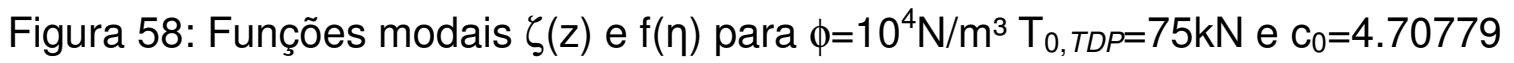

Figura 59: Deslocamento horizontal do $T D P x_{c}$ e velocidade $v_{c}$ para $\phi=10^{4} \mathrm{~N} / \mathrm{m}^{3}$, $\mathrm{T}_{0, T D P}=50 \mathrm{kN}, \xi=10 \%, \mathrm{c}_{0}=4.70779, \mathrm{~T}=3.0 \mathrm{~s}$ para $\mathrm{x}_{\mathrm{c}, 0}=0.1$ e $\mathrm{v}_{\mathrm{c}, 0}=0.1$ 98

Figura 60: Mapa de fase e mapa de Poincaré para $\phi=10^{4} \mathrm{~N} / \mathrm{m}^{3}, \mathrm{~T}_{0, T D P}=50 \mathrm{kN}$, $\xi=10 \%, c_{0}=4.70779, \mathrm{~T}=3.0 \mathrm{~s}$ para $\mathrm{x}_{\mathrm{c}, 0}=0.1$ e $\mathrm{v}_{\mathrm{c}, 0}=0.1$ 98

Figura 61: Funções modais $\zeta(z)$ e $f(\eta)$ para $\phi=10^{5} \mathrm{~N} / \mathrm{m}^{3} \mathrm{~T}_{0, \text { TDP }}=40 \mathrm{kN}$ e $\mathrm{c}_{0}=9.35991$ 
Figura 62: Deslocamento horizontal do $T D P \mathrm{x}_{\mathrm{c}}$ e velocidade $\mathrm{v}_{\mathrm{c}}$ para $\phi=10^{5} \mathrm{~N} / \mathrm{m}^{3}$, $T_{0, T D P}=40 \mathrm{kN}, \xi=10 \%, \mathrm{c}_{0}=9.35991, \mathrm{~T}=4.5 \mathrm{~s}$ para $\mathrm{x}_{\mathrm{c}, 0}=0.1$ e $\mathrm{v}_{\mathrm{c}, 0}=0.07$. 99

Figura 63: Mapa de fase e mapa de Poincaré para $\phi=10^{5} \mathrm{~N} / \mathrm{m}^{3}, T_{0, T D P}=40 \mathrm{kN}$, $\xi=10 \%, c_{0}=9.35991, T=4.5$ s para $x_{c, 0}=0.1$ e $v_{c, 0}=0.07$ 99

Figura 64: Funções modais $\zeta(z)$ e f(n) para $\phi=10^{5} \mathrm{~N} / \mathrm{m}^{3} \mathrm{~T}_{0, T D P}=50 \mathrm{kN}$ e $\mathrm{c}_{0}=8.37176$ 100

Figura 65: Deslocamento horizontal do $T D P x_{c}$ e velocidade $v_{c}$ para $\phi=10^{5} \mathrm{~N} / \mathrm{m}^{3}$, $\mathrm{T}_{0, T D P=50 \mathrm{kN},} \xi=10 \%, \mathrm{c}_{0}=8.37176, \mathrm{~T}=3.1 \mathrm{~s}$ para $\mathrm{x}_{\mathrm{c}, 0}=0.1$ e $\mathrm{v}_{\mathrm{c}, 0}=0.1$ 100

Figura 66: Mapa de fase e mapa de Poincaré para $\phi=10^{5} \mathrm{~N} / \mathrm{m}^{3}, T_{0, T D P}=50 \mathrm{kN}$, $\xi=10 \%, c_{0}=8.37176, T=3.1$ s para $x_{c, 0}=0.1$ e $v_{c, 0}=0.1$ 100

Figura 67: Funções modais $\zeta(z)$ e f(n) para $\phi=10^{4} \mathrm{~N} / \mathrm{m}^{3} \mathrm{~T}_{0, T D P}=20 \mathrm{kN}$ e $\mathrm{c}_{0}=7.44366$

Figura 68: Deslocamento horizontal do $T D P x_{c}$ e velocidade $v_{c}$ para $\phi=10^{4} \mathrm{~N} / \mathrm{m}^{3}$, $T_{0, T D P}=20 \mathrm{kN}, \xi=20 \%, \mathrm{c}_{0}=7.44366, \mathrm{~T}=10.2 \mathrm{~s}$ para $\mathrm{x}_{\mathrm{c}, 0}=0.1$ e $\mathrm{v}_{\mathrm{c}, 0}=0.03$. 101

Figura 69: Mapa de fase e mapa de Poincaré para $\phi=10^{4} \mathrm{~N} / \mathrm{m}^{3}, T_{0, T D P}=20 \mathrm{kN}$, $\xi=20 \%, c_{0}=7.44366, T=10.2$ s para $x_{c, 0}=0.1$ e $v_{c, 0}=0.03$

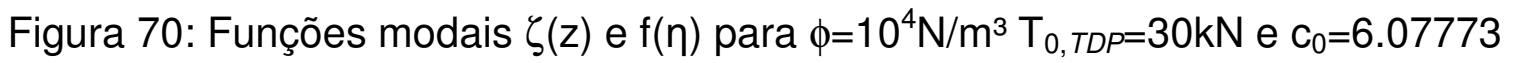

Figura 71: Deslocamento horizontal do $T D P x_{c}$ e velocidade $v_{c}$ para $\phi=10^{4} \mathrm{~N} / \mathrm{m}^{3}$, $\mathrm{T}_{0, T D P}=30 \mathrm{kN}, \xi=20 \%, \mathrm{c}_{0}=6.07773, \mathrm{~T}=8.1 \mathrm{~s}$ para $\mathrm{x}_{\mathrm{c}, 0}=0.1$ e $\mathrm{v}_{\mathrm{c}, 0}=0.04$. 102

Figura 72: Mapa de fase e mapa de Poincaré para $\phi=10^{4} \mathrm{~N} / \mathrm{m}^{3}, T_{0, T D P}=30 \mathrm{kN}$, $\xi=20 \%, c_{0}=6.07773, T=8.1 \mathrm{~s}$ para $x_{c, 0}=0.1$ e $v_{c, 0}=0.04$ 102

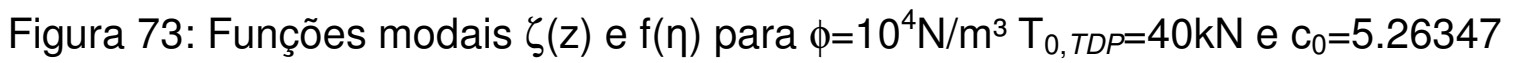

Figura 74: Deslocamento horizontal do $T D P x_{c}$ e velocidade $v_{c}$ para $\phi=10^{4} \mathrm{~N} / \mathrm{m}^{3}$, $\mathrm{T}_{0, T D P}=40 \mathrm{kN}, \xi=20 \%, \mathrm{c}_{0}=5.26347, \mathrm{~T}=4.1 \mathrm{~s}$ para $\mathrm{x}_{\mathrm{c}, 0}=0.1$ e $\mathrm{v}_{\mathrm{c}, 0}=0.08$. 103

Figura 75: Mapa de fase e mapa de Poincaré para $\phi=10^{4} \mathrm{~N} / \mathrm{m}^{3}, T_{0, T D P}=40 \mathrm{kN}$, $\xi=20 \%, c_{0}=5.26347, T=4.1 \mathrm{~s}$ para $\mathrm{x}_{\mathrm{c}, 0}=0.1$ e $\mathrm{v}_{\mathrm{c}, 0}=0.08$ 103

Figura 76: Funções modais $\zeta(\mathrm{z})$ e f(n) para $\phi=10^{4} \mathrm{~N} / \mathrm{m}^{3} \mathrm{~T}_{0, T D P}=75 \mathrm{kN}$ e $\mathrm{c}_{0}=4.70779$ 
Figura 77: Deslocamento horizontal do $T D P \mathrm{x}_{\mathrm{c}}$ e velocidade $\mathrm{v}_{\mathrm{c}}$ para $\phi=10^{4} \mathrm{~N} / \mathrm{m}^{3}$, $\mathrm{T}_{0, T D P}=50 \mathrm{kN}, \xi=20 \%, \mathrm{c}_{0}=4.70779, \mathrm{~T}=3.0 \mathrm{~s}$ para $\mathrm{x}_{\mathrm{c}, 0}=0.1$ e $\mathrm{v}_{\mathrm{c}, 0}=0.1$ 104

Figura 78: Mapa de fase e mapa de Poincaré para $\phi=10^{4} \mathrm{~N} / \mathrm{m}^{3}, T_{0, T D P}=50 \mathrm{kN}$, $\xi=20 \%, c_{0}=4.70779, \mathrm{~T}=3.0 \mathrm{~s}$ para $\mathrm{x}_{\mathrm{c}, 0}=0.1$ e $\mathrm{v}_{\mathrm{c}, 0}=0.1$ 104

Figura 79: Funções modais $\zeta(\mathrm{z})$ e $\mathrm{f}(\eta)$ para $\phi=10^{5} \mathrm{~N} / \mathrm{m}^{3} \mathrm{~T}_{0, T D P}=40 \mathrm{kN}$ e $\mathrm{c}_{0}=9.35991$ 105

Figura 80: Deslocamento horizontal do $T D P x_{c}$ e velocidade $v_{c}$ para $\phi=10^{5} \mathrm{~N} / \mathrm{m}^{3}$, $\mathrm{T}_{0, T D P}=40 \mathrm{kN}, \xi=20 \%, \mathrm{c}_{0}=9.35991, \mathrm{~T}=4.5 \mathrm{~s}$ para $\mathrm{x}_{\mathrm{c}, 0}=0.1$ e $\mathrm{v}_{\mathrm{c}, 0}=0.07$. 105

Figura 81: Mapa de fase e mapa de Poincaré para $\phi=10^{5} \mathrm{~N} / \mathrm{m}^{3}, T_{0, T D P}=40 \mathrm{kN}$, $\xi=20 \%, c_{0}=9.35991, T=4.5$ s para $x_{c, 0}=0.1$ e $v_{c, 0}=0.07$ 105

Figura 82: Funções modais $\zeta(z)$ e f(n) para $\phi=10^{5} \mathrm{~N} / \mathrm{m}^{3} \mathrm{~T}_{0, T D P}=50 \mathrm{kN}$ e $\mathrm{c}_{0}=8.37176$ 106

Figura 83: Deslocamento horizontal do $T D P x_{c}$ e velocidade $v_{c}$ para $\phi=10^{5} \mathrm{~N} / \mathrm{m}^{3}$, $\mathrm{T}_{0, T D P=50 \mathrm{kN},} \xi=20 \%, \mathrm{c}_{0}=8.37176, \mathrm{~T}=3.1 \mathrm{~s}$ para $\mathrm{x}_{\mathrm{c}, 0}=0.1$ e $\mathrm{v}_{\mathrm{c}, 0}=0.1$ 106

Figura 84: Mapa de fase e mapa de Poincaré para $\phi=10^{5} \mathrm{~N} / \mathrm{m}^{3}, T_{0, T D P}=50 \mathrm{kN}$,

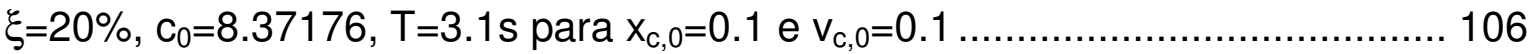

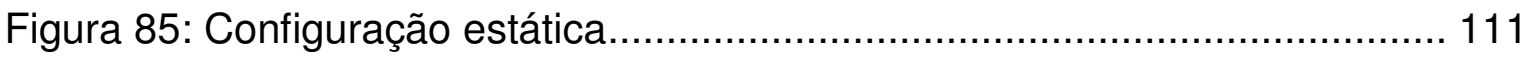

Figura 86: Solução completa em diferentes instantes ...................................... 111

Figura 87: Força Normal na configuração estática $(\mathrm{N})$................................... 112

Figura 88: Força Normal em diferentes instantes $(\mathrm{N})$.................................... 112

Figura 89: Momento Fletor na configuração estática $(\mathrm{Nm})$............................... 113

Figura 90: Momento Fletor em diferentes instantes $(\mathrm{Nm})$............................... 113

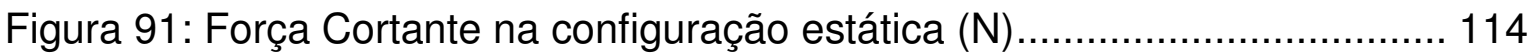

Figura 92: Força Cortante em diferentes instantes $(\mathrm{N})$................................... 114

Figura 93: Tensões Normais na configuração estática $\left(\mathrm{N} / \mathrm{m}^{2}\right)$......................... 115

Figura 94: Tensões Normais em diferentes instantes $\left(\mathrm{N} / \mathrm{m}^{2}\right)$....................... 115

Figura 95: Tensão Cisalhante na configuração estática $\left(\mathrm{N} / \mathrm{m}^{2}\right) \ldots \ldots \ldots \ldots \ldots \ldots \ldots \ldots \ldots . . . . . . . .116$

Figura 96: Tensão Cisalhante em diferentes instantes $\left(\mathrm{N} / \mathrm{m}^{2}\right)$...................... 116

Figura 97: Mapa de fase para $T_{0, T D P}=495.1 \mathrm{kN}$ e $T_{\text {MOD. ORDEM REDUZIDA }}=0.44 \mathrm{~s} \ldots . .119$

Figura 98: Mapa de fase para $T_{0, T D P}=324.7 \mathrm{kN}$ e $T_{\text {MOD. ORDEM REDUZIDA }}=1.05 \mathrm{~s} \ldots . .119$

Figura 99: Mapa de fase para $T_{0, T D P}=125.0 \mathrm{kN}$ e $T_{\text {MOD. ORDEM REDUZIDA }}=0.40 \mathrm{~s} \ldots . .119$

Figura 100: Mapa de fase para $T_{0, T D P}=35 \mathrm{kN}$ e $T_{\text {MOD. ORDEM REDUZIDA }}=12.4 \mathrm{~s} \ldots \ldots . .122$ 
Figura 101: Mapa de fase para $T_{0, T D P}=50 \mathrm{kN}$ e $T_{\text {MOD. ORDEM REDUZIDA }}=6.0 \mathrm{~s}$

Figura 102: Mapa de fase para $T_{0, T D P}=60 \mathrm{kN}$ e $T_{\text {MOD. ORDEM REDUZIDA }}=6.6 \mathrm{~s}$

Figura 103: Mapa de fase obtido com o programa Orcaflex para $T_{0, T D P}=35 \mathrm{kN}$ e

TTREM DE ONDAS $=6.2 \mathrm{~s}$

Figura 104: Mapa de fase obtido com o programa Orcaflex para $T_{0, T D P}=50 \mathrm{kN} \mathrm{e}$

TTREM DE ONDAS $=3.0 \mathrm{~s}$

Figura 105: Mapa de fase obtido com o programa Orcaflex para $T_{0, T D P}=60 \mathrm{kN}$ e

TTREM DE ONDAS $=3.3 \mathrm{~s}$

Figura 106: Comparação da configuração estática.

Figura 107: Modelo de decaimento da tração ao longo da linha

Figura 108: Comparação entre os modelos de decaimento da tração 139 


\section{Lista de Tabelas}

Tabela 1: Freqüências modais adimensionais $(\omega)$ em função da posição do TDP $\left(\mathrm{c}_{0}\right)$ 55

Tabela 2: Propriedades físicas da viga considerada no estudo de caso de análise paramétrica, ver Pesce et al [28].

Tabela 3: Modos de vibração em função da rigidez do solo e tração estática no TDP.

Tabela 4: Resumo dos processamentos na abordagem teórica do estudo de caso 88

Tabela 5: Tabela de comparação entre freqüência adimensional do modelo original e freqüência adicional do modelo de ordem reduzida 90

Tabela 6: Propriedades físicas do riser considerado no estudo de caso de análise paramétrica, ver Pesce et al [28]. 92

Tabela 7: Modos de vibração obtidos para viga sob flexão simples. 93

Tabela 8: Resumo dos processamentos P1 a P6, com $\xi=10 \%$, na aplicação do modelo a risers em catenária

Tabela 9: Resumo dos processamentos P7 a P12, com $\xi=30 \%$, na aplicação do modelo a risers em catenária 107

Tabela 10: Dados de entrada para comparação com programa Poliflex 3D ...... 118

Tabela 11: Resultados do processamento sem excitação paramétrica 120

Tabela 12: Resultados do processamento com Poliflex 3D sem excitação paramétrica

Tabela 13: Dados de entrada para comparação com programa Orcaflex.

Tabela 14: Resultados do processamento com excitação paramétrica 124

Tabela 15: Resultados do processamento com Ocaflex com excitação paramétrica

Tabela 16: Resultados de processamentos com programa Poliflex 3D 141

Tabela 17: Tabela resumo com período e altura do trem de ondas na Bacia de Campos, de acordo com levantamento em Silva e Nogueira [34]. 


\section{Lista de Símbolos}

$A_{i} \quad$ coeficientes para definição de $R_{i}$ e $S_{i}$

$a_{i} \quad$ coeficientes do modelo de ordem reduzida

$\hat{a}_{i} \quad$ coeficientes do modelo de ordem reduzida, divididos por $a_{0}$ (massa modal equivalente)

$B_{i} \quad$ coeficientes para definição de $R_{i}$ e $S_{i}$

$b_{i} \quad$ coeficientes do modelo de ordem reduzida

$\hat{b}_{i} \quad$ coeficientes do modelo de ordem reduzida, divididos por $a_{0}$ (massa modal equivalente)

$c(\tau) \quad$ abscissa axial adimensional que define a posição do ponto de contato ou touch down point (TDP)

$c_{0}$ posição do ponto de contato ou touch down point (TDP) da solução de equilíbrio estático

$c_{1} \quad$ posição do ponto de contato ou touch down point (TDP) da solução de equilíbrio dinâmico

$C_{i} \quad$ coeficientes para definição de $R_{i}$ e $S_{i}$

$D_{k}{ }^{p} \quad$ operador para p-ésima derivada k-ésima temporal

E módulo de Young do material

$H \quad$ função de Heavyside

I momento de inércia da seção transversal

$K_{i j} \quad$ coeficientes para correlação entre $R_{3}$ e $p_{1}$ (analogamente para $S_{3}$ e $\left.q_{1}\right)$

$L \quad$ comprimento em que a tração no cabo, no trecho apoiado, decai a valores suficientemente baixos

$p \quad$ peso da viga ou peso submerso do riser

$p_{1} \quad$ condição inicial do problema

$q_{1} \quad$ condição inicial do problema

$r_{1} \quad$ condição inicial do problema

$R_{i} \quad$ coeficientes utilizados na solução $r_{1}$

$s_{1} \quad$ condição inicial do problema

$S_{i} \quad$ coeficientes utilizados na solução $s_{1}$ 


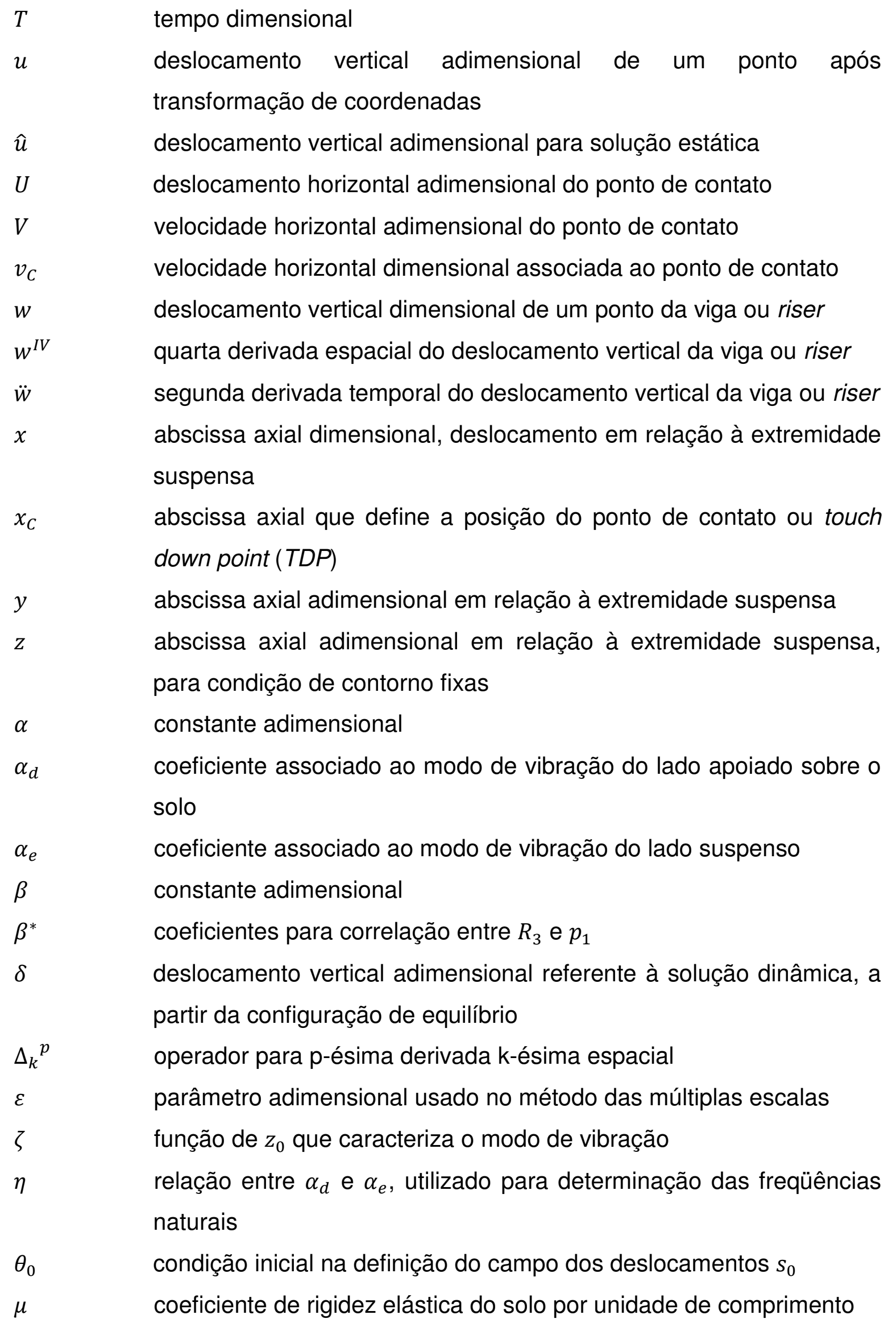


coeficiente de rigidez elástica do solo por unidade de área deslocamento vertical adimensional de um ponto do elemento quarta derivada espacial do deslocamento vertical adimensional de um ponto

segunda derivada temporal do deslocamento vertical adimensional de um ponto

coeficiente de amortecimento

massa linear do elemento

tempo adimensional

freqüência de vibração do modo, na formulação adimensional com flexão simples

freqüência de vibração do modo, na formulação adimensional com efeito da tração

freqüência de oscilação vertical da plataforma, na formulação adimensional 


\section{Resumo}

Este trabalho visa a avaliar os efeitos dinâmicos em vigas esbeltas semiinfinitas com contato unilateral em apoio elástico sob flexão composta, e sua possível aplicação a dutos flexíveis de extração de petróleo offshore em configuração de catenária (Steel Catenary Risers) provocados por esforços de movimento da plataforma.

O estudo se desenvolve com formulação de vigas semi-infinitas em duas dimensões (2D) com suporte unilateral elástico, considerando-se inicialmente apenas efeito de flexão, à qual se aplica o método das múltiplas escalas (MME) para obtenção dos modos de vibração, e posteriormente o método das variedades invariantes (MVI) para recuperação das relações modais entre as coordenadas generalizadas e as variáveis modais.

Trata-se, a seguir, da formulação do problema com consideração da tração, tanto estática como dinâmica, e a projeção da equação de movimento completa segundo os modos obtidos no problema da flexão simples, para obtenção do modelo de ordem reduzida com efeito de tração, que permitiu estudar este último qualitativa e quantitativamente.

A formulação inicial teve como ponto de partida a utilização de mudanças de variáveis que permitiu a transformação do problema de condições móveis em condições fixas de contorno, propiciando caracterizar o movimento vertical da viga na solução analítica do problema de vibrações livres, até o limite possível, para depois utilizar técnicas de integração numérica na solução do problema de vibrações forçadas.

A motivação para estudo de tais efeitos está ligada à importância indiscutível que tem para a indústria petrolífera e para a economia mundial, além do crescente papel deste segmento em termos nacionais. A descoberta do Campo de Tupi, na Bacia de Santos, que representa a maior reserva de óleo e gás do País, alavancou pesquisas para extração de petróleo em águas ultra- 
profundas (profundidades da ordem de 2.500m) (fonte: www.petrobras.com.br), além da necessidade já estabelecida de maiores pesquisas em águas profundas (profundidades que podem chegar a $2.000 \mathrm{~m}$ ).

Considere-se também a motivação da própria Petrobrás, cujo intuito é ter capacidade de extrair 4,5 milhões de barris por dia até 2020. Tal capacidade de produção deve exigir 45 sistemas de produção, sendo que cada sistema de produção necessita de 4 a 5 barcos de apoio, totalizando assim 200 barcos operando em 2020 (Fonte: O Estado de São Paulo, Ano 131, № 42786, Coluna Celso Ming).

Apesar da crescente pesquisa em fontes alternativas de energia, a demanda não só por óleo e gás, mas também pela utilização de polímeros e componentes plásticos, ainda sustentará a extração de petróleo por no mínimo algumas décadas. Além disso, técnicas melhores na fixação de carbono (técnicas naturais e artificiais para seqüestro de carbono), permitem sonhar que a utilização de petróleo, desde a sua queima até a produção de material sintético possa se tornar cada vez menos impactante para o meio ambiente. 


\section{Abstract}

This work aims at analyzing the dynamical effects in slender reticulated beams on elastic support with unilateral contact under combined axial force and bending, and the possibility of its association with steel catenary risers connected to offshore structures, caused by movement of the platform.

The study is developed based on 2D semi-infinite beams on unilateral elastic support, initially considering only bending effects, to which the multiple scales method (MSM) is applied to obtain vibration modes, and thereafter the invariant manifold method (IMM) to obtain the modal relationships between the generalized coordinates and the modal variables. It follows the formulation of the problem considering tension effects, both from static and dynamical sources, and the projection of the full equation of motion with respect to the vibration modes from the first formulation considering only bending effects, which allows us to obtain the reduced order model with tension effects, and its application to steel catenary risers $(\mathrm{SCR})$.

The starting point for the mathematical formulation is a variable transformation, which allows us to change a free boundary into a fixed boundary one. It is then possible to obtain the free-vibration analytical solution, as far as possible, and then use numerical integration schemes to obtain the forced vibration time responses.

As motivation, we can firstly mention the unquestionable role of the oil and gas industry for the world economy, besides its increasing market share within the national economy. The discovery of the Tupi Field, in the Santos Basin, which represents the biggest oil and gas reserve of the Country, gave impulse to investments and research in all fields associated to the oil and gas area, in ultra deep water (depth around $2.500 \mathrm{~m}$ ), besides the well known need of research in deep water (depth around $2.000 \mathrm{~m}$ ). 
In spite of the growing research on alternative sources of energy, the world still demands not only oil and gas, but also polymers and plastic components, and this will still be the scenario for the next decades. Besides that, the increasing of knowledge in carbon sequestration may keep up the use of oil and gas for some more decades, hopefully with less environment impact. 


\section{Capítulo 1}

\subsection{Introdução}

Do ponto de vista de modelagem matemática, o presente trabalho, na pesquisa sobre o comportamento dinâmico de uma viga esbelta em vibrações livres, está intrinsecamente dissociado do conceito de modo de vibração clássico, já que o contato unilateral, parte integrante do problema, introduz condições móveis de contorno e acarreta nós e ventres em posição variável no tempo.

Um dos resultados surpreendentes que se pode verificar, que evidencia tratar-se de um problema não clássico, é o número finito de modos de vibração no modelo contínuo, cuja dimensão é infinita, ao invés de um número infinito de modos. Tal resultado difere da maioria dos encontrados na bibliografia, nos quais, ao se modelar um sistema contínuo, obtém-se um numero infinito de modos de vibração.

Tradicionalmente, o problema do contato unilateral é abordado por métodos computacionais, como, por exemplo, aqueles em que se utiliza o Método dos Elementos Finitos (MEF), ou o Método da Camada Limite, no qual se modela a linha como cabo e posteriormente faz-se a correção para o trecho em que há efeito significativo de flexão. O presente trabalho busca uma abordagem analítica, na qual se considera o esforço de flexão desde o início do problema, além de levar em consideração a variação da posição do ponto de contato no tempo.

Do ponto de vista tecnológico e econômico, a relevância do petróleo na economia mundial dispensa apresentação. No entanto, foi com a descoberta do Campo de Tupi na Bacia de Santos que o Brasil ganhou atenção ímpar no cenário mundial. Apesar das reservas se encontrarem em grandes profundidades (da ordem de $6.000 \mathrm{~m}$, dos quais pelo menos $2.000 \mathrm{~m}$ representam lâmina d'água), a quantidade de petróleo existente justifica desenvolver técnicas que possam levar o País à condição de um dos maiores produtores mundiais. 
Neste cenário, os equipamentos e as técnicas de análise tradicionalmente utilizados deverão sofrer desenvolvimento tecnológico para responder às novas exigências e necessidades de uma exploração complexa, ainda inédita em escala industrial no cenário mundial.

O uso de risers em catenária, ou steel catenary risers (SCR), ganhou importância na medida em que a profundidade de onde o petróleo deverá ser extraído aumentou consideravelmente. Com a perspectiva que a prospecção em águas profundas e ultra profundas apenas aumente, a demanda por tecnologia e compreensão dos fenômenos de interação entre os risers e o mar serão tendência no cenário científico nacional e internacional.

O uso de $S C R$ 's para extração de petróleo em lâminas d'água menores vem sendo feito há algum tempo, mas muitos fenômenos ainda são preocupantes, como fadiga. O fato de o SCR estar sujeito a carregamentos ambientais faz com que esforços repetitivos sejam continuados na vida útil do elemento estrutural. As possibilidades de movimentação incluem o ponto de contato com o solo, Touch Down Point, ou apenas TDP, que está sempre associado a problemas de fadiga.

As regiões de conexão do riser com a plataforma flutuante (por exemplo, as do tipo Floating Production Storage and Offloading, ou FPSO) também requerem especial atenção. A conexão do tubo com a unidade flutuante é uma estrutura de sustentação (hangoff) e uma junta flexível, cujo papel é absorver as variações de momento geradas pelas movimentações impostas por ondas, conexão esta que também desperta preocupação por problemas de fadiga.

Os seis graus de liberdade que uma estrutura flutuante possui, na terminologia de língua inglesa, são: surge, que é o movimento de translação longitudinal, sway (ou drift) o lateral, e heave, o vertical; roll é a rotação em torno de eixo longitudinal, picht é a rotação em torno de eixo transversal, e yaw a rotação em torno de eixo vertical. 


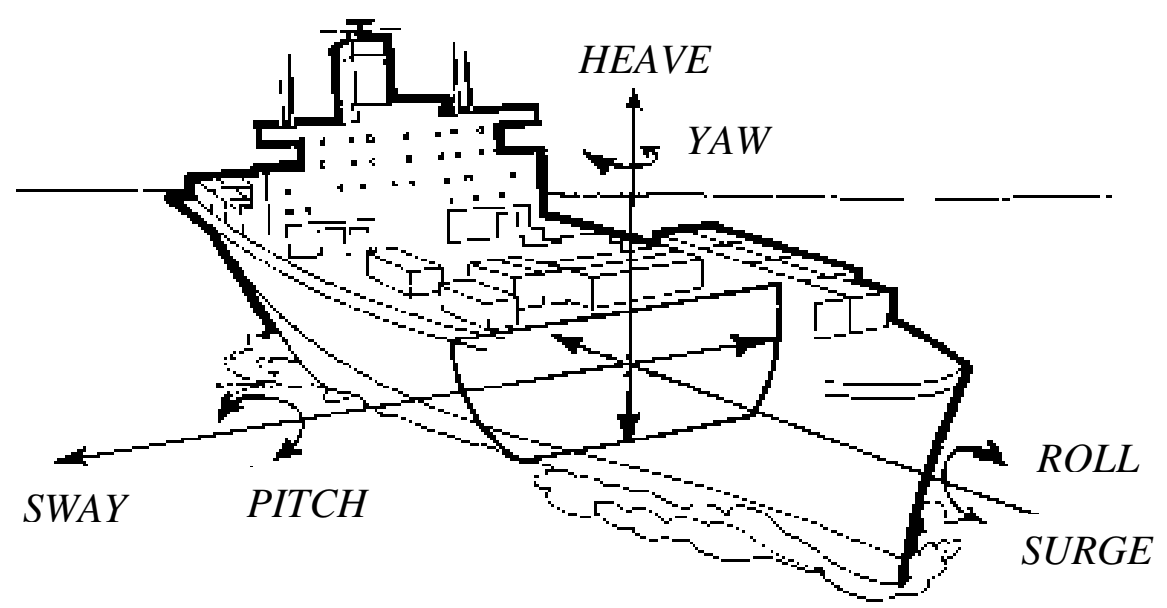

Figura 1: Os 6 graus de liberdade de uma plataforma offshore (Fonte: Association of Marine Underwriters of San Francisco, www.amusf.com)

No caso dos risers, os carregamentos mais relevantes são de natureza fluidodinâmica, sendo que especial atenção é dada aos esforços gerados por ondas de superfície, correnteza oceânica e vento. A ação de ondas gera, entre outros, o efeito de heave, e atua como movimento imposto para o riser. A correnteza exerce influência na posição da unidade flutuante no plano da linha d'água, o que gera alterações na configuração estática dos risers, além de poder provocar vibrações induzidas por vórtices (VIV). A ação do vento acontece de maneira indireta, mas também gera movimentação da plataforma no plano da linha d'água.

Além de todos estes efeitos, não se pode esquecer o carregamento gerado por fluxo interno de óleo, gás, ou ambos, que pode ser de extrema relevância em algumas condições. 


\subsection{Justificativa}

A importância do petróleo para a economia mundial por si só justifica o investimento no desenvolvimento científico e o largo espectro de pesquisas sobre todos os fenômenos associados à atividade e que podem representar risco à viabilidade da operação, à vida humana e ao ecossistema.

O acidente envolvendo a companhia British Petroleum no Golfo do México em 2010, um dos maiores desastres ecológicos da história, com vazamento diário estimado em 5.000 barris de petróleo por dia (800.000 litros), mostra o grau de complexidade para tratar problemas tecnológicos em águas profundas. Neste caso específico, pode-se citar o custo associado à perda material gerada pela explosão no poço de Macondo, que terminou por afundar a sonda Deepwater Horizon, que custou US\$560 milhões em sua construção. O desastre também matou 11 pessoas, e foi atribuído a falhas mecânicas e humanas, incluindo certo descaso nos processos de manutenção.

Somente no ano de 2009, houve 28 importantes vazamentos de petróleo e de gás na parte americana do Golfo do México. No Mar do Norte britânico, houve 85 incidentes sérios, e na Noruega, 37 casos similares (Fonte: O Estado de São Paulo, Ano 132, № 42838, Artigo Norman Gall).

A segurança associada ao processo de extração justifica melhoria constante em todas as ferramentas utilizadas, desde as ferramentas de análise dos componentes estruturais a instrumentos de medição. 


\subsection{Objetivos}

O primeiro objetivo deste trabalho é desenvolver uma metodologia na forma de um modelo matemático de ordem reduzida para uma viga esbelta semiinfinita com contato unilateral em apoio elástico sob flexão composta, que permita estudar os efeitos dinâmicos gerados por movimento imposto ou variação da tração na extremidade.

A partir deste modelo, pretende-se avaliar as possibilidades de aplicação a risers em catenária, essencialmente na descrição do comportamento dinâmico na zona de contato, ou Touch Down Zone (TDZ), frente à movimentação ou variação de esforços aplicados na extremidade.

Apesar dos 6 graus de liberdade existentes em uma plataforma offshore (no caso de plataformas flutuantes ou mistas), três de translação (surge, sway e heave) e três de rotação (roll, pitch e yaw), como mostrado na Fig. 1, o estudo da aplicação a risers em catenária preocupou-se no presente texto, inicialmente, apenas com as respostas causadas por movimentos no plano do mar, impostos no topo, decorrentes de heave e surge.

De posse dos modos de vibração obtidos e da descrição da topologia modal, utilizou-se o método de Galerkin para projeção da representação do problema dinâmico no espaço de fase unidimensional do modelo de ordem reduzida. Apesar de simplificador, este caminho permitiu que se interpretassem algumas características essenciais do problema, como a possibilidade de variação da tração ao longo do elemento no trecho apoiado.

Por fim, a integração da equação não linear de movimento do modelo de ordem reduzida com consideração do efeito da tração é feita utilizando-se o método Runge Kutta de $4^{\underline{a}}$ ordem, obtendo-se os históricos para deslocamento horizontal do TDP e sua velocidade, e conseqüentemente o mapa de fase. 
Para a aplicação da metodologia ao estudo dos risers flexíveis em catenária, o estudo da dinâmica global foi feito com base no programa Poliflex 3D, v. 2.0.1., Martins et al [20], 2008. O presente trabalho aborda a questão da dinâmica local, especificamente da região do TDZ.

Imagina-se que esta metodologia possa também ser utilizada no estudo de vórtices induzidos por vibração (VIV), que também implicaria condição de tração dinâmica, considerada nesta formulação, embora com outras amplitudes e freqüências, dependentes do "lock in" dos desprendimentos de vórtice, e da vibração modal do trecho suspenso do riser. $O$ foco do presente trabalho, no entanto, é o estudo dos efeitos decorrentes de heave e surge.

No desenvolvimento do trabalho, preferiu-se arcar com as limitações dos modos de vibração obtidos no modelo de flexão simples, garantindo-se, porém, que o modelo de ordem reduzida utilizado seja capaz de considerar a tração e sua variação ao longo da linha, contato unilateral elástico, e a principal fonte de não linearidades do problema, advinda da transformação do problema de condições de contorno móveis em fixas. 


\subsection{Metodologia e hipóteses}

A Fig. 2 abaixo ilustra aspectos globais do problema:

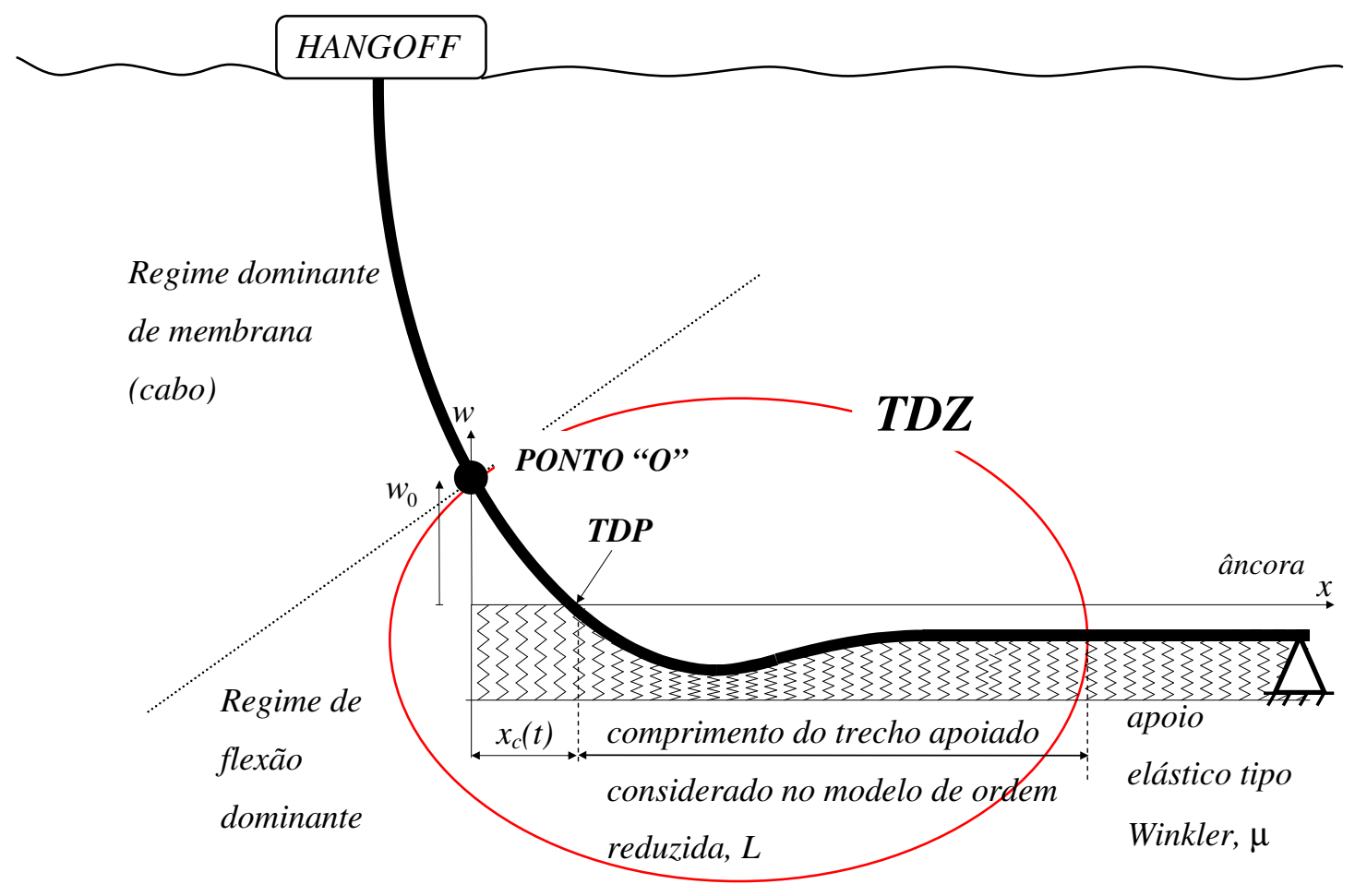

Figura 2: Representação esquemática dos aspectos globais do problema

Por conveniência metodológica, procedeu-se ao estudo analítico e numérico de uma viga semi-infinita parcialmente apoiada sobre apoio elástico. $\mathrm{O}$ modelo utilizado, com características fortemente não-lineares (devido ao contato unilateral), apresentou o desafio de lidar com condições de contorno móveis, devido à variação da posição do ponto de contato ao longo do tempo.

A solução adotada para superar o problema e transformá-lo em um de condições de contorno fixas foi aplicar uma transformação de variáveis de maneira a impor que a abscissa do ponto de contato da viga com o solo correspondesse sempre ao valor nulo, garantindo-se, ainda, que o ponto "O" da Fig. 2 continuasse fixo nas novas coordenadas. 
Para a obtenção da solução foi utilizada a técnica de modelagem de ordem reduzida de um grau de liberdade (MOR), de maneira que ainda fosse possível obter respostas satisfatórias qualitativa e quantitativamente. A utilização de um modelo de baixa hierarquia deve-se à maior simplicidade para um primeiro estudo a respeito destes fenômenos, mas, também, permite abordagens analíticas e estudos paramétricos capazes de caracterizar o comportamento e a estabilidade da resposta dinâmica. A metodologia desenvolvida utilizou também o método de Galerkin para projetar a resposta dinâmica do problema com consideração do efeito da flexo-tração segundo os modos de vibração do modelo de flexão simples.

As hipóteses gerais, utilizadas no desenvolvimento de todo o trabalho, são:

- Análise feita em duas dimensões (2D), restrita à região do Touch Down Zone (TDZ);

- Contato unilateral elástico, com consideração de superfície plana;

- Apenas a dinâmica vertical foi considerada no trecho dominado pelo regime de flexão;

- A dinâmica do trecho sob regime dominante de membrana, ou seja, a análise global do problema, foi estudada à parte, fornecendo condições de carregamento $(T(t))$ na interface $O$ da Fig. 2;

- Consideração de linearidade física, partindo do princípio que a viga é muito esbelta e a relação $w_{0} /\left(x_{c}+L\right)$, ou seja, a razão entre o deslocamento vertical e o comprimento horizontal considerado é pequena;

De fato, o primeiro modelo, que nos permitiu a obtenção dos modos, trata da formulação com flexão simples, desconsiderando os efeitos da tração. Essa escolha deveu-se ao fato que o desenvolvimento analítico do problema, com consideração direta dos efeitos de tração, mostrou-se de grande complexidade. Nesta primeira etapa, procurou-se determinar o equilíbrio para o modelo em questão. A Fig. 3 abaixo ilustra o modelo físico utilizado: 


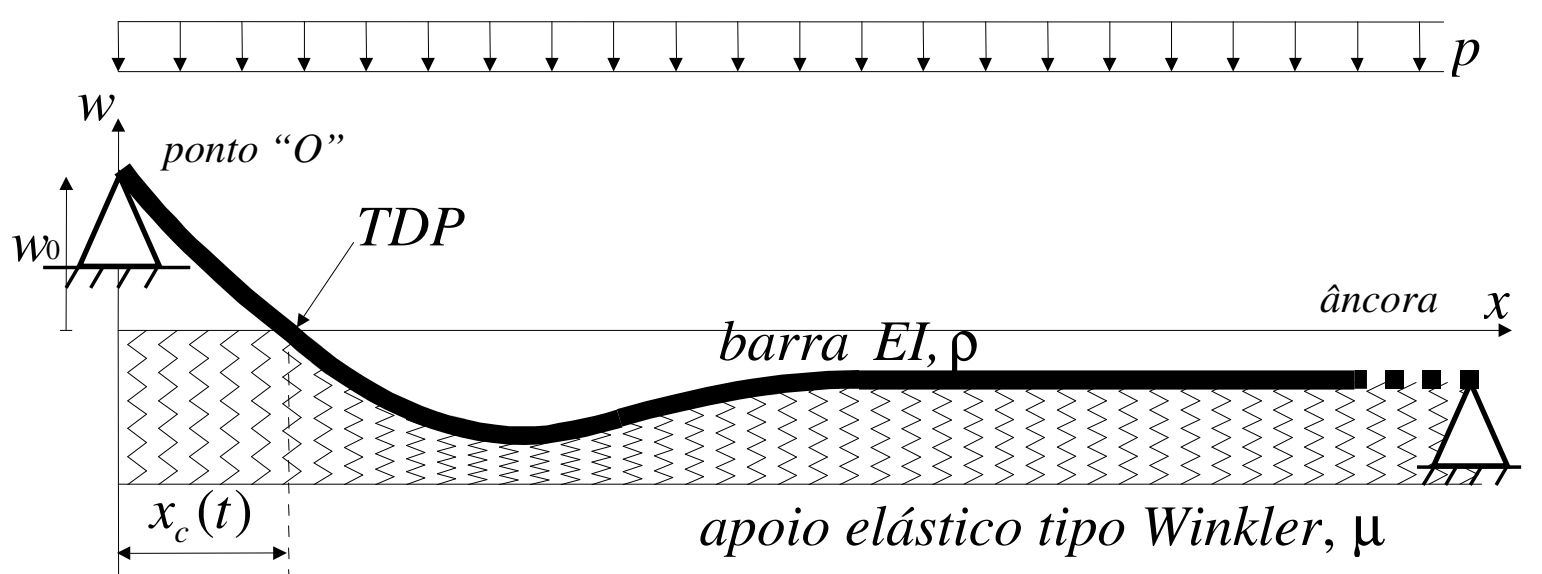

Figura 3: Viga esbelta semi-infinita com contato unilateral em apoio elástico sob flexão simples

As hipóteses específicas para esta etapa são:

- O ponto "O" é indeslocável, e a viga é modelada como sendo semi-infinita;

- A tração ao longo da linha é muito baixa, $T(x) \cong 0$;

- A curvatura está linearizada, de acordo com a teoria elementar de barra, sendo que a não linearidade fica restrita ao contato unilateral.

Os resultados provenientes desta etapa, na forma de output do problema, são a configuração estática do trecho suspenso, $\hat{u}(z)$, e a posição $c_{0}$ do TDP.

A este equacionamento, seguiu-se com a aplicação do Método das Múltiplas Escalas (MME), numa generalização do método proposto por Nayfeh e Mook [27], método assintótico de perturbação onde a idéia principal é a transformação de equações diferenciais não lineares do problema em uma série de equações lineares com solução obtida por expansão assintótica. Neste método, os termos de ordem superior representam uma pequena correção frente aos termos de ordem inferior. O MME é tradicionalmente utilizado em escalas temporais, mas no caso deste estudo foi aplicado também à escala espacial, por imposição da formulação do problema.

Apesar das características não-lineares do problema, a aplicação do MME levou em consideração apenas os termos de ordem $\varepsilon$ para obtenção dos modos, 
ou seja, os modos não-lineares estão caracterizados pelos termos de primeira ordem.

A aplicação do MME ao modelo com flexão simples permitiu a obtenção dos modos, e interessantes constatações quanto aos finitos modos de vibração da estrutura (e não infinitos, como usualmente obtido na análise de modelos contínuos).

As hipóteses específicas nesta etapa são as mesmas da etapa anterior, isto é:

- O ponto "O" é indeslocável, e a viga é modelada como sendo semi-infinita;

- A tração ao longo da linha, tanto estática como dinâmica, é baixa, $T(x, t) \cong$ 0 ;

- A curvatura está linearizada, de acordo com a teoria elementar de barra, sendo que a não linearidade fica restrita ao contato unilateral.

O resultado proveniente desta etapa, na forma de output do problema, são as funções modais $\zeta(z)$, tanto para o trecho suspenso como para o trecho apoiado sobre o solo.

Decorre daí a descrição da topologia modal (variedade invariante) a partir das relações modais.

$\mathrm{Na}$ terceira etapa do problema, onde se pretende obter a resposta dinâmica, relacionou-se a movimentação imposta ao ponto "O" com a introdução de uma força normal a ser aplicada na extremidade suspensa, como mostra o esquema da Fig. 4 abaixo: 

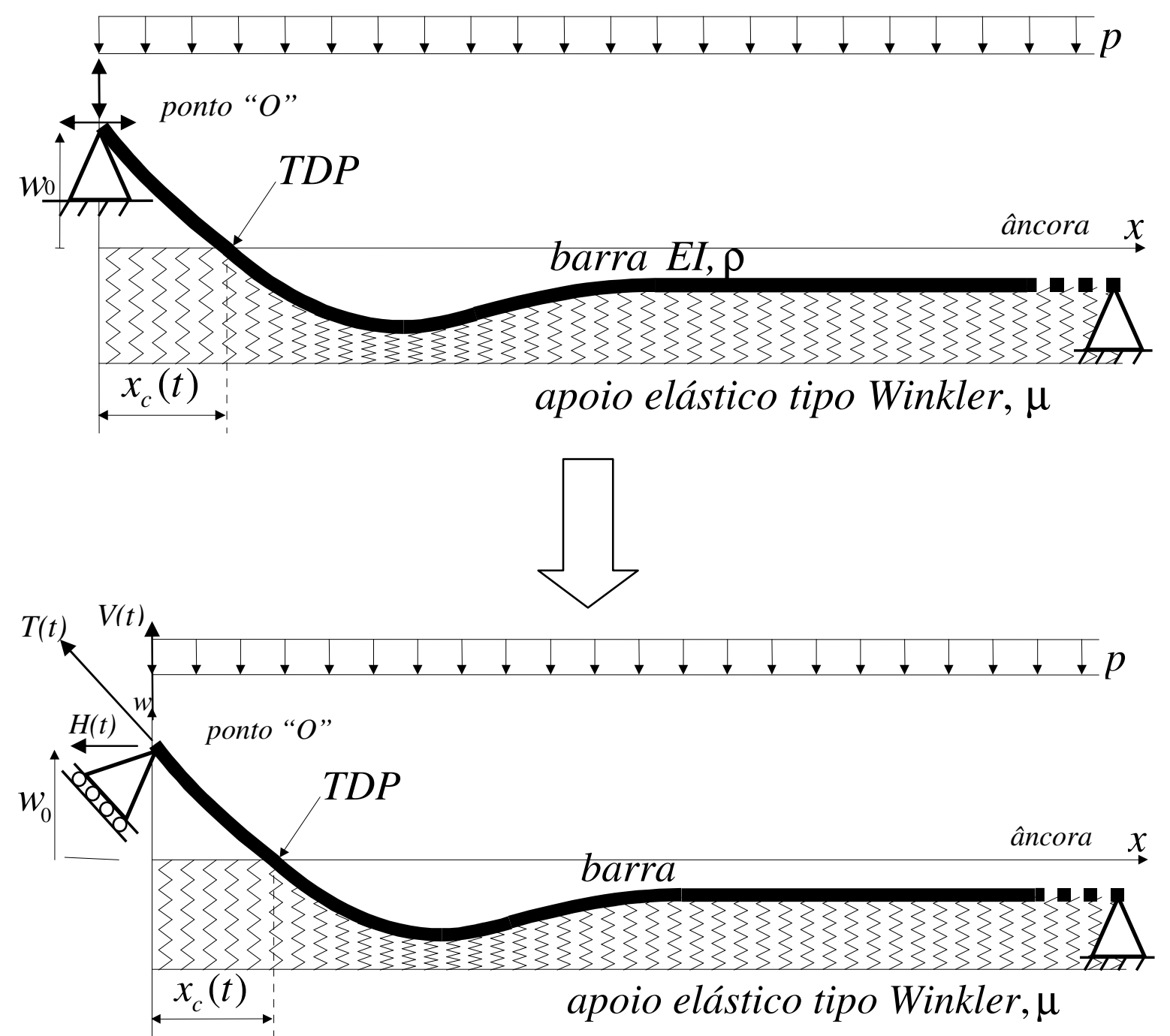

Figura 4: Viga esbelta semi-infinita com contato unilateral em apoio elástico sob flexão composta com baixa tração

As hipóteses específicas para esta etapa são:

- Configuração estática $\hat{u}(z)$ e funções modais $\zeta(z)$ do problema em que há tração baixa são semelhantes aos do problema sem tração (flexão simples);

- Possibilidade de aplicação da projeção segundo o método de Galerkin, das funções $\hat{u}(z)$ e $\zeta(z)$;

- A tração dinâmica $T(x, t)=\left[T_{0}+T_{1} \cos (\Omega t)\right] e^{-\theta x}$ é introduzida como hipótese "ad hoc", uma vez que a estática/dinâmica horizontal não está sendo discutida na formulação do problema; 
- Decaimento do parâmetro $\theta$ da expressão do carregamento dinâmico calibrado para $T(x, t)$ tendendo a zero, em trecho semelhante ao da hipótese de atrito Coulomb, a ser discutido oportunamente.

O desenvolvimento desta etapa segue através dos seguintes passos:

- Integração numérica no domínio do tempo;

- Análise paramétrica variando: tração estática $T_{0}$ no ponto "O", amplitude de tração dinâmica $T_{1}$ no ponto "O", freqüência adimensional $\Omega$ do carregamento forçado, amortecimento equivalente linear $\xi$, rigidez do solo $\varphi$ e as condições iniciais;

- Leva-se em consideração que a resposta de todo o sistema é a soma da parcela do deslocamento estático com o dinâmico $u(z, \tau)=\hat{u}(z)+\delta(z, \tau)$, sendo $\delta(z, \tau)=\zeta(z) \cdot U(\tau)$ e $c(\tau)=c_{0}+U(\tau)$, onde $c_{0}$ é a posição adimensional do ponto de contato e $U(\tau)$ é a variável modal.

Nesta etapa, obtém-se:

- O modelo de ordem reduzida (na forma de um oscilador não linear forçado);

$\mathrm{Na}$ etapa que segue, desenvolve-se a modelagem de viga esbelta com contato unilateral em apoio elástico sob regime de flexão composta com baixa tração para modelagem de riser de aço em catenária (SCR).

As hipóteses específicas nesta etapa são:

- Dados de entrada decorrentes de análise global efetuada no programa Poliflex 3D: tração estática $T_{0}$ no ponto "O", tração dinâmica $T_{1}$ no ponto "O" e deslocamento inicial $x_{0}$ do TDP. Note-se que os valores da tração estática $T_{0}$ são relativamente baixos, já que o ângulo junto ao hangoff é de aproximadamente $70^{\circ}$; 
- Os parâmetros geométricos e mecânicos são típicos de risers (SCR): profundidade, rigidez flexional $E I$, rigidez à tração $E A$, peso próprio submerso $p$, massa por unidade de comprimento $\rho$ (incluindo a massa adicional), rigidez do solo por unidade de área $\varphi$, amortecimento equivalente linear $\xi$ e estado de mar (heave e surge);

- As condições iniciais são próximas às do movimento do TDP, segundo o programa Poliflex 3D;

- O deslocamento na configuração estática obtido no modelo analítico, $\hat{u}(z)$, é similar ao deslocamento na configuração estática obtido através do programa Poliflex 3D (vide Anexo 7.2).

Nesta etapa, após integração numérica no domínio do tempo, obtém-se:

- Modelo de ordem reduzida, que permite avaliar casos sob e sem ressonância paramétrica;

- E as seguintes saídas dos processamentos:

- Deslocamento dinâmico: $\delta(z, \tau)=\zeta(z) \cdot U(\tau)$;

- Deslocamento dinâmico do TDP: $c(\tau)=c_{0}+U(\tau)$;

- Deslocamento total, somando-se a posição na configuração estática ao deslocamento dinâmico de cada ponto da Touch Down Zone $(T D Z): u(z, \tau)=\hat{u}(z)+\delta(z, \tau)$;

- Associação do deslocamento total com a coordenada dimensional através da relação $u(z, \tau) \Rightarrow w(x, t)$, o que permite recuperar todos os esforços atuantes na estrutura no domínio considerado, culminando com os esforços de máxima tensão;

- Comparação com o programa Poliflex 3D (e Orcaflex) e discussão das respostas, para os casos com e sem ressonância paramétrica. 


\subsection{Revisão Bibliográfica}

O presente estudo teve como base o trabalho de Mazzilli e Lenci [22], que desenvolve a modelagem analítica do problema da flexão simples de uma viga semi-infinita com contato unilateral elástico, introduzindo mudança de coordenadas, que transforma o problema de condições de contorno móveis em fixas. A abordagem permitiu avaliar as condições adotadas para aplicação do MME, numa generalização da técnica proposta por Nayfeh e Mook [27], utilizando um modelo de baixa hierarquia, para obtenção dos modos de vibração da estrutura em função da posição do TDP, usada como variável modal.

Em Demeio e Lenci [16], foi possível avaliar algumas sugestões para carregamento imposto na extremidade livre do cabo suspenso, bem como a utilização de séries de potência para obtenção da perturbação dinâmica frente à solução estática do problema.

Em Baracho Neto [5], foi baseado o estudo da recuperação das variedades invariantes a partir da resposta dinâmica das coordenadas generalizadas em vibração livre.

O trabalho desenvolvido em Sanches [31], permitiu averiguar formulações para os efeitos de heave e VIV, bem como o tratamento dado a coeficientes utilizados como características físicas dos materiais.

Em Meneghini [26], e Pesce [29], pôde-se conhecer diferentes abordagens para o riser em catenária quanto a esforços causados por ações ambientais, bem como a comparação de modelagem matemática com resultados obtidos em laboratório. Este estudo foi importante para familiarização com o tema e aprofundamento nos conhecimentos sobre a indústria offshore e algumas de suas principais características.

As análises contidas em Aranha et al [1], [2] e [3], Chatjigeorgiou [7], [8], [9], Chatjigeorgiu [10], Fujarra [17], Josefsson [19], Pesce et al [28], Ramos e 
Pesce [30], Sanches et al [32], Silveira [35], Simos e Fujarra [36], e Takafuji [39], permitiram conhecer a ordem de grandeza de alguns dados de entrada do problema, como características físicas do riser e diferentes abordagens já propostas.

O trabalho desenvolvido em Silva e Nogueira [34] foi utilizado como referência na obtenção de dados de comportamento de mar para os casos de aplicação prática, no tocante ao período do trem de ondas e à altura associada a cada um destes períodos. 


\subsection{Organização do Texto}

O capítulo 2 aborda o estudo dos modos não lineares de vibração e a obtenção das relações modais da viga esbelta semi-infinita com contato unilateral em apoio elástico, sob flexão simples. Tem início a formulação com modelo em 2D de barra, na caracterização do problema de condições de contorno móveis. Introduz-se uma mudança de variáveis para obtenção de formulação analítica com condições de contorno fixas, e chega-se à equação diferencial representativa para o problema. A partir daí procede-se à determinação e caracterização das propriedades dos modos de vibração, bem como o resgate das relações modais, que permitem a descrição topológica dos modelos de ordem reduzida a serem posteriormente estudados no capítulo 3.

O capítulo 3 aborda o estudo da resposta dinâmica de viga esbelta semiinfinita com contato unilateral elástico, sob flexão e tração variável espacial e temporalmente. De posse dos modos de vibração obtidos através do modelo com flexão simples (capítulo 2), faz-se, mediante o método de Galerkin, a projeção da equação de movimento segundo estes modos na obtenção do modelo de ordem reduzida.

O capítulo 4 trata do estudo de caso para o modelo de ordem reduzida obtido no capítulo 3 , na forma de análise paramétrica e também na forma de aplicação ao estudo da dinâmica do riser na $T D Z$, utilizando dados de entrada segundo bibliografia e programas de análise específico, típicos da área offshore. A aplicação a risers trata das solicitações oriundas de movimentação no topo da plataforma, heave e surge. Neste contexto, apenas um amortecimento viscoso linear equivalente foi considerado para simular tanto o estrutural quanto o fluido, embora se saiba que o amortecimento fluido dinâmico é mais corretamente modelado de forma não-linear. $\mathrm{Na}$ integração numérica do modelo de ordem reduzida foi utilizado o método Runge-Kutta de $4^{\underline{a}}$ ordem. Desenvolve-se 0 capítulo com a obtenção dos esforços na viga/riser, essencialmente os de momento fletor e força cortante, e conseqüentemente tensão de flexão e tensão cisalhante, tanto para a configuração estática como em diferentes instantes do 
tempo na análise dinâmica. Por fim, comparam-se os resultados de deslocamento obtidos com outras ferramentas disponíveis, com 0 intuito de valorizar a contribuição da nova abordagem e discutir limitações de cada uma das formulações propostas.

O capítulo 5 discute os resultados obtidos ao longo do texto, desde o capítulo 2 até o capítulo 4, as limitações dos modelos e métodos utilizados e as possibilidades de melhoria em trabalhos futuros, tanto do ponto de vista de descrição do modelo físico como desenvolvimento do modelo matemático. 
2. Capítulo 2: Modos não-lineares de vibração na flexão simples para viga esbelta semi-infinita com contato unilateral em apoio elástico

\subsection{Formulação do problema e condições de contorno móveis}

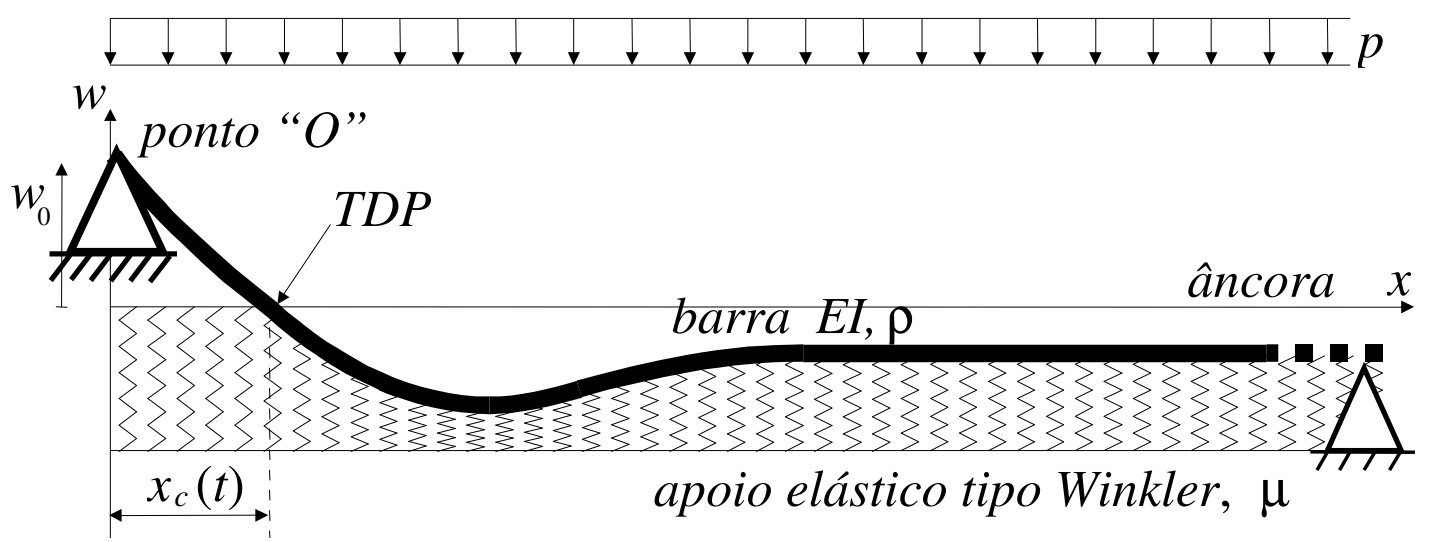

Figura 5: Viga esbelta sob flexão simples com contato unilateral em apoio elástico

A equação para o modelo estudado de barra semi-infinita, inicialmente jazendo sobre um meio elástico horizontal, com coeficiente de rigidez $\mu$, tendo massa por unidade de comprimento $\rho$ (incluindo a massa adicional), sob carregamento uniformemente distribuído $p$, submetida a flexão simples oriunda da imposição de um deslocamento vertical, conforme se apresenta na Fig. 5, pode ser expressa da seguinte forma:

$E I \frac{\partial^{4} w}{\partial x^{4}}+\rho \frac{\partial^{2} w}{\partial t^{2}}+p=0$

para $0<x<x_{c}$, $\mathrm{e}$

$E I \frac{\partial^{4} w}{\partial x^{4}}+\mu w+\rho \frac{\partial^{2} w}{\partial t^{2}}+p=0$

para $x>x_{c}$, 
sendo $x_{c}$ a posição do ponto de contato, ou simplesmente touch down point $(T D P)$.

Note-se que as não-linearidades geométricas da teoria de barras foram ignoradas, conforme se depreende da linearização da curvatura, $\left(\partial^{4} w / \partial x^{4}\right)$ implícita em (1) e (2), uma vez que as não-linearidades oriundas do contato são muito mais fortes.

Portanto, (1) e (2) traduzem o equilíbrio dinâmico na direção vertical do modelo da teoria elementar de barras com apoio unilateral elástico, ao qual se introduz a força aparente de inércia, segundo o princípio de D’Alambert.

Com o intuito de escrever as equações acima na forma adimensional, definem-se as seguintes relações:

$y=\alpha x, \quad v=\frac{\mu}{p} w, \quad \tau=\beta t, \quad \alpha=\sqrt[4]{\frac{\mu}{4 E I}}, \quad$ e $\quad \beta=\sqrt{\frac{\mu}{\rho}}$.

Aplicando-se as relações acima, chegamos às equações adimensionais que descrevem o problema:

$\frac{1}{4} \frac{\partial^{4} v}{\partial y^{4}}+\frac{\partial^{2} v}{\partial \tau^{2}}+1=0$

para $0<y<c(\tau), c(\tau)=\alpha x_{c}(t), \mathrm{e}$

$\frac{1}{4} \frac{\partial^{4} v}{\partial y^{4}}+\frac{\partial^{2} v}{\partial \tau^{2}}+v+1=0$

para $y>c(\tau)$.

Neste contexto, a posição do TDP fica definida como sendo aquela em que $v[c(\tau), \tau]=0$. 
Com o intuito de transformar o problema acima, de condições de contorno móveis, em um de condições de contorno fixas, faz-se uso da seguinte transformação:

$z=\frac{y}{c(\tau)}-1 \Rightarrow y=(z+1) c(\tau)$

e uma nova função é definida:

$u(z, \tau)=v(y, \tau)$

Doravante será adotada no prosseguimento deste trabalho a notação ('), "linha", para representar as derivadas espaciais em relação à $z$, e a notação (·), "ponto", para representar as derivadas temporais em relação à $\tau$.

Assim, reescrevem-se as equações (4) e (5), com a consideração da equação (6), e com ajuda da função de Heavyside $(H(z))$ (deduções constam no anexo 7.1) como:

$$
\begin{aligned}
& \frac{1}{4} u^{I V}-2 \dot{u}^{\prime} \dot{c}(\mathrm{z}+1) c^{3}+u^{\prime \prime} \dot{c}^{2}(1+z)^{2} c^{2}+u^{\prime}(z+1)\left(2 \dot{c}^{2}-\ddot{c} c\right) c^{2}+c^{4} \ddot{u}+H c^{4} u+c^{4} \\
& =0
\end{aligned}
$$

sendo:

$$
\begin{aligned}
& H(z)=0, \quad-1<z<0 \\
& H(z)=1, \quad z>0 .
\end{aligned}
$$

As condições de contorno para o problema ficam definidas através das equações:

$$
\begin{aligned}
& u(-1, \tau)=\frac{\mu}{p} w_{0} \\
& u^{\prime \prime}(-1, \tau)=0
\end{aligned}
$$


$u_{e}(0, \tau)=0$

$u_{d}(0, \tau)=0$

$u_{e}^{\prime}(0, \tau)-u_{d}^{\prime}(0, \tau)=0$

$u^{\prime \prime}{ }_{e}(0, \tau)-u_{d}^{\prime \prime}(0, \tau)=0$

$u^{\prime \prime \prime}{ }_{e}(0, \tau)-u^{\prime \prime \prime}{ }_{d}(0, \tau)=0$

sendo decorrentes de: deslocamento vertical dimensional imposto $w_{0}$ na extremidade suspensa; momento fletor igual zero na extremidade suspensa; deslocamento vertical nulo à esquerda e à direita do TDP; rotação à esquerda igual à rotação à direita do TDP; momento fletor à esquerda igual ao momento fletor à direita do TDP; e força cortante à esquerda igual à força cortante à direita do TDP.

As notações $u_{e}$ e $u_{d}$ e suas derivadas referem-se à respectiva função calculada à esquerda e à direita do TDP, conforme se apresenta na Fig. 5 , isto é, o trecho à esquerda é o suspenso, e à direita é o trecho em contato com o meio elástico.

Recorde-se que, para a viga semi-infinita, não há reflexão de onda de $z=+\infty$ para $z=0$.

Note-se que as equações segmentadamente lineares por domínio (4) e (5) com condições de contorno móveis, foram substituídas pela equação não-linear (8) com condições de contorno fixas. 


\subsection{Obtenção dos modos não lineares de vibração}

A solução do problema pode ser escrita na forma:

$u(z, \tau)=\hat{u}(z)+\delta(z, \tau)$,

sendo $\hat{u}(z)$ a solução correspondente à configuração estática e $\delta(z, \tau)$ os deslocamentos adicionais dinâmicos, tal que a solução $\hat{u}(z)$ deva satisfazer a:

$\frac{1}{4} \hat{u}^{I V}+c_{0}{ }^{4}+H c_{0}{ }^{4} \hat{u}=0$,

onde $c_{0}$ define a posição do TDP da configuração de equilíbrio estático. A partir daí temos que:

$\hat{u}_{e}(z)=-\frac{c_{0}{ }^{4}}{6} z^{4}+\frac{c_{0}{ }^{3}}{3}\left(1-c_{0}\right) z^{3}+c_{0}{ }^{3} z^{2}-c_{0}\left(1+c_{0}\right) z$, para $-1<z<0$,

e

$\hat{u}_{d}(z)=\left[\cos \left(c_{0} z\right)-c_{0} \sin \left(c_{0} z\right)\right] e^{-c_{0} z}-1$, para $z>0$.

A posição do $T D P, c_{0}$, para a viga na configuração estática, fica definida pela solução da equação:

$\frac{c_{0}^{4}}{6}+\frac{2 c_{0}^{3}}{3}+c_{0}^{2}+c_{0}-\hat{u}_{0}=0$ 


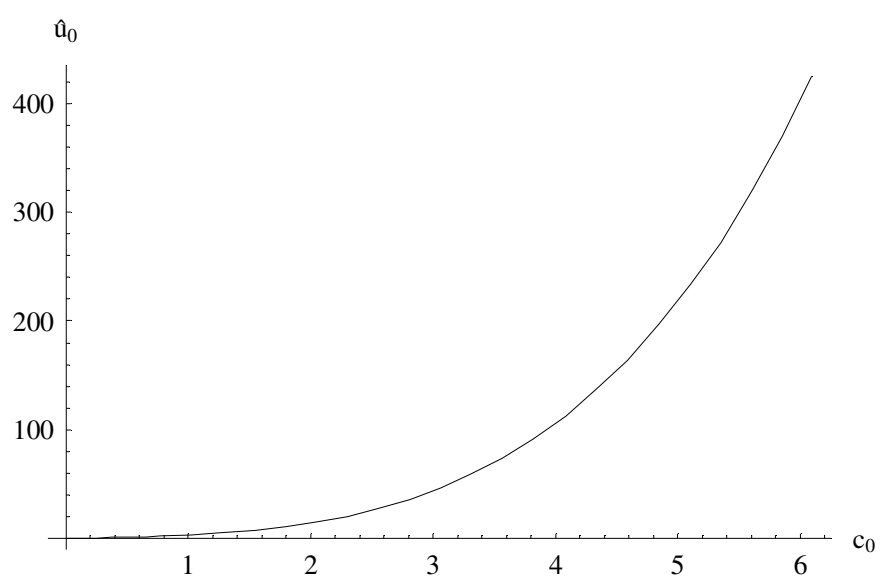

Figura 6: Deslocamento estático imposto na extremidade do trecho suspenso û. em função da posição do $\operatorname{TDP}\left(\mathrm{c}_{0}\right)$

Substituindo (11) em (8) e considerando a equação (12), chega-se à equação diferencial dos deslocamentos dinâmicos verticais adimensionais com condições de contorno fixas:

$$
\begin{array}{r}
\frac{1}{4} \delta^{I V}-2 \dot{\delta}^{\prime} \dot{c}(\mathrm{z}+1) c^{3}+\hat{u}^{\prime \prime} \dot{c}^{2}(1+z)^{2} c^{2}+\delta^{\prime \prime} \dot{c}^{2}(1+z)^{2} c^{2}+\hat{u}^{\prime}(z+1)\left(2 \dot{c}^{2}-\ddot{c} c\right) c^{2} \\
+\delta^{\prime}(z+1)\left(2 \dot{c}^{2}-\ddot{c} c\right) c^{2}+c^{4} \ddot{\delta}+H c^{4} \delta+\left(c^{4}-c_{0}{ }^{4}\right)(1+H \hat{u})=0 .
\end{array}
$$

A partir das condições de contorno (10) chega-se às condições de contorno para os deslocamentos dinâmicos:

$$
\begin{aligned}
& \delta(-1, \tau)=0 \\
& \delta^{\prime \prime}(-1, \tau)=0 \\
& \delta_{e}(0, \tau)=0 \\
& \delta_{d}(0, \tau)=0 \\
& \delta_{e}^{\prime}(0, \tau)-\delta_{d}^{\prime}(0, \tau)=0 \\
& \delta^{\prime \prime}{ }_{e}(0, \tau)-\delta^{\prime \prime}{ }_{d}(0, \tau)=0 \\
& \delta^{\prime \prime \prime}{ }_{e}(0, \tau)-\delta^{\prime \prime \prime}{ }_{d}(0, \tau)=0
\end{aligned}
$$

sendo respectivamente: deslocamento dinâmico vertical na extremidade suspensa igual a zero; momento fletor dinâmico igual a zero na extremidade suspensa; deslocamento dinâmico vertical nulo à esquerda e à direita do TDP; rotação 
dinâmica à esquerda igual à rotação dinâmica à direita do TDP; momento fletor dinâmico à esquerda igual ao momento fletor dinâmico à direita do TDP; e força cortante dinâmica à esquerda igual à força cortante dinâmica à direita do TDP.

Note-se que as condições de contorno propostas em (10) e (17) desconsideram a movimentação da extremidade do trecho suspenso (ponto "O"). Considera-se que uma melhoria do modelo possa ser obtida considerando-se estes efeitos na formulação do problema, eventualmente na forma de uma excitação de suporte.

A solução para a equação (16) é feita através da aplicação do método das múltiplas escalas $\mathrm{MME}$, sendo necessária uma generalização da técnica apresentada em Nayfeh e Mook [27], para contemplar, além das escalas temporais, também as espaciais, com a seguinte expansão assintótica, usado o parâmetro de perturbação $0<\varepsilon \ll 1$ :

$\delta=\varepsilon \delta_{1}\left(z_{0}, z_{1}, \ldots, \tau_{0}, \tau_{1}, \ldots\right)+\varepsilon^{2} \delta_{2}\left(z_{0}, z_{1}, \ldots, \tau_{0}, \tau_{1}, \ldots\right)+\varepsilon^{3} \delta_{3}\left(z_{0}, z_{1}, \ldots, \tau_{0}, \tau_{1}, \ldots\right)+\cdots$
$c=c_{0}+\varepsilon c_{1}\left(\tau_{0}, \tau_{1}, \ldots\right)+\varepsilon^{2} c_{2}\left(\tau_{0}, \tau_{1}, \ldots\right)+\varepsilon^{3} c_{3}\left(\tau_{0}, \tau_{1}, \ldots\right)+\cdots$

sendo $k=1,2,3, \ldots, \quad z_{k}=\varepsilon^{k} z$ as escalas espaciais e $\tau_{k}=\varepsilon^{k} \tau$ as escalas temporais. Os operadores diferenciais em relação às variáveis independentes $z_{k}$ $\tau_{k}$ são introduzidos a seguir:

$$
\begin{aligned}
& \frac{\partial}{\partial z}=\Delta_{0}+\varepsilon \Delta_{1}+\varepsilon^{2} \Delta_{2}+\cdots \\
& \frac{\partial^{2}}{\partial z^{2}}=\Delta_{0}{ }^{2}+2 \varepsilon \Delta_{0} \Delta_{1}+\varepsilon^{2}\left(\Delta_{1}{ }^{2}+2 \Delta_{0} \Delta_{2}\right)+\cdots \\
& \frac{\partial^{3}}{\partial z^{3}}=\Delta_{0}{ }^{3}+3 \varepsilon \Delta_{0}{ }^{2} \Delta_{1}+\varepsilon^{2}\left(3 \Delta_{0} \Delta_{1}{ }^{2}+3 \Delta_{0}{ }^{2} \Delta_{2}\right)+\cdots \\
& \frac{\partial^{4}}{\partial z^{4}}=\Delta_{0}^{4}+4 \varepsilon \Delta_{0}^{3} \Delta_{1}+\varepsilon^{2}\left(6 \Delta_{0}{ }^{2} \Delta_{1}{ }^{2}+4 \Delta_{0}{ }^{3} \Delta_{2}\right)+\cdots \\
& \frac{\partial}{\partial \tau}=D_{0}+\varepsilon D_{1}+\varepsilon^{2} D_{2}+\cdots \\
& \frac{\partial^{2}}{\partial \tau^{2}}=D_{0}{ }^{2}+2 \varepsilon D_{0} D_{1}+\varepsilon^{2}\left(D_{1}{ }^{2}+2 D_{0} D_{2}\right)+\cdots
\end{aligned}
$$


sendo $\Delta_{k}^{p}=\frac{\partial^{p}}{\partial z_{k}{ }^{p}}$ e $D_{k}^{p}=\frac{\partial^{p}}{\partial \tau_{k}{ }^{p}}$.

Substituindo as equações (18) e (19) em (16), vem:

$$
\begin{aligned}
& \frac{1}{4}\left[\Delta_{0}{ }^{4}+4 \varepsilon \Delta_{0}{ }^{3} \Delta_{1}+\varepsilon^{2}\left(6 \Delta_{0}{ }^{2} \Delta_{1}{ }^{2}+4 \Delta_{0}{ }^{3} \Delta_{2}\right)+\cdots\right]\left(\varepsilon \delta_{1}+\varepsilon^{2} \delta_{2}+\cdots\right) \\
& +\left(1+z_{0}\right)^{2}\left(c_{0}+\varepsilon c_{1}+\varepsilon^{2} c_{2}+\varepsilon^{3} c_{3}\right. \\
& +\cdots)^{2}\left[\left(D_{0}+\varepsilon D_{1}+\varepsilon^{2} D_{2}+\cdots\right)\left(c_{0}+\varepsilon c_{1}+\varepsilon^{2} c_{2}+\varepsilon^{3} c_{3}+\cdots\right)\right]^{2}\left(\Delta_{0}{ }^{2}\right. \\
& \left.+2 \varepsilon \Delta_{0} \Delta_{1}+\varepsilon^{2}\left(\Delta_{1}{ }^{2}+2 \Delta_{0} \Delta_{2}\right)+\cdots\right) \hat{u}\left(z_{0}\right) \\
& +\left(1+z_{0}\right)^{2}\left(c_{0}+\varepsilon c_{1}+\varepsilon^{2} c_{2}+\varepsilon^{3} c_{3}\right. \\
& +\cdots)^{2}\left[\left(D_{0}+\varepsilon D_{1}+\varepsilon^{2} D_{2}+\cdots\right)\left(c_{0}+\varepsilon c_{1}+\varepsilon^{2} c_{2}+\varepsilon^{3} c_{3}+\cdots\right)\right]^{2}\left(\Delta_{0}{ }^{2}\right. \\
& \left.+2 \varepsilon \Delta_{0} \Delta_{1}+\varepsilon^{2}\left(\Delta_{1}{ }^{2}+2 \Delta_{0} \Delta_{2}\right)+\cdots\right)\left(\varepsilon \delta_{1}+\varepsilon^{2} \delta_{2}+\varepsilon^{3} \delta_{3}+\cdots\right) \\
& -2\left(1+z_{0}\right)\left(c_{0}+\varepsilon c_{1}+\varepsilon^{2} c_{2}+\varepsilon^{3} c_{3}+\cdots\right)^{3}\left[( D _ { 0 } + \varepsilon D _ { 1 } + \varepsilon ^ { 2 } D _ { 2 } + \cdots ) \left(c_{0}+\varepsilon c_{1}+\varepsilon^{2} c_{2}\right.\right. \\
& \left.\left.+\varepsilon^{3} c_{3}+\cdots\right)\right]\left[( D _ { 0 } + \varepsilon D _ { 1 } + \varepsilon ^ { 2 } D _ { 2 } + \cdots ) ( \Delta _ { 0 } + \varepsilon \Delta _ { 1 } + \varepsilon ^ { 2 } \Delta _ { 2 } + \cdots ) \left(\varepsilon \delta_{1}\right.\right. \\
& \left.\left.+\varepsilon^{2} \delta_{2}+\varepsilon^{3} \delta_{3}+\cdots\right)\right] \\
& +\left(1+z_{0}\right)\left\{2 \left(c_{0}+\varepsilon c_{1}+\varepsilon^{2} c_{2}+\varepsilon^{3} c_{3}\right.\right. \\
& +\cdots)^{2}\left[\left(D_{0}+\varepsilon D_{1}+\varepsilon^{2} D_{2}+\cdots\right)\left(c_{0}+\varepsilon c_{1}+\varepsilon^{2} c_{2}+\varepsilon^{3} c_{3}+\cdots\right)\right]^{2} \\
& -\left(c_{0}+\varepsilon c_{1}+\varepsilon^{2} c_{2}+\varepsilon^{3} c_{3}+\cdots\right)^{3}\left(D_{0}^{2}+2 \varepsilon D_{0} D_{1}+\varepsilon^{2}\left(D_{1}^{2}+2 D_{0} D_{2}\right)\right. \\
& \left.+\cdots)\left(c_{0}+\varepsilon c_{1}+\varepsilon^{2} c_{2}+\varepsilon^{3} c_{3}+\cdots\right)\right\} \Delta_{0} \hat{u}\left(z_{0}\right) \\
& +\left(1+z_{0}\right)\left\{2 \left(c_{0}+\varepsilon c_{1}+\varepsilon^{2} c_{2}+\varepsilon^{3} c_{3}\right.\right. \\
& +\cdots)^{2}\left[\left(D_{0}+\varepsilon D_{1}+\varepsilon^{2} D_{2}+\cdots\right)\left(c_{0}+\varepsilon c_{1}+\varepsilon^{2} c_{2}+\varepsilon^{3} c_{3}+\cdots\right)\right]^{2} \\
& -\left(c_{0}+\varepsilon c_{1}+\varepsilon^{2} c_{2}+\varepsilon^{3} c_{3}+\cdots\right)^{3}\left(D_{0}{ }^{2}+2 \varepsilon D_{0} D_{1}+\varepsilon^{2}\left(D_{1}{ }^{2}+2 D_{0} D_{2}\right)\right. \\
& \left.+\cdots)\left(c_{0}+\varepsilon c_{1}+\varepsilon^{2} c_{2}+\varepsilon^{3} c_{3}+\cdots\right)\right\}\left(\Delta_{0}+\varepsilon \Delta_{1}+\varepsilon^{2} \Delta_{2}+\cdots\right)\left(\varepsilon \delta_{1}+\varepsilon^{2} \delta_{2}\right. \\
& \left.+\varepsilon^{3} \delta_{3}+\cdots\right) \\
& +\left(c_{0}+\varepsilon c_{1}+\varepsilon^{2} c_{2}+\varepsilon^{3} c_{3}+\cdots\right)^{4}\left(D_{0}{ }^{2}+2 \varepsilon D_{0} D_{1}+\varepsilon^{2}\left(D_{1}{ }^{2}+2 D_{0} D_{2}\right)+\cdots\right)\left(\varepsilon \delta_{1}+\varepsilon^{2} \delta_{2}\right. \\
& \left.+\varepsilon^{3} \delta_{3}+\cdots\right) \\
& +H\left(c_{0}+\varepsilon c_{1}+\varepsilon^{2} c_{2}+\varepsilon^{3} c_{3}+\cdots\right)^{4}\left(\varepsilon \delta_{1}+\varepsilon^{2} \delta_{2}+\varepsilon^{3} \delta_{3}+\cdots\right) \\
& +\left[\left(c_{0}+\varepsilon c_{1}+\varepsilon^{2} c_{2}+\varepsilon^{3} c_{3}+\cdots\right)^{4}-c_{0}^{4}\right](1+H \hat{u})=0 .
\end{aligned}
$$

No presente estudo, apenas os termos de ordem $\varepsilon$ serão analisados, devendo-se, portanto, fazer: 
$\frac{1}{4} \Delta_{0}{ }^{4} \delta_{1}+c_{0}{ }^{4} D_{0}{ }^{2} \delta_{1}+H c_{0}{ }^{4} \delta_{1}-c_{0}{ }^{3}\left(1+z_{0}\right)\left(D_{0}{ }^{2} c_{1}\right)\left(\Delta_{0} \hat{u}\right)+4 c_{0}{ }^{3} c_{1}(1+H \hat{u})=0$,

tal que a solução possa ser escrita na forma:

$\delta_{1}=r_{1}\left(z_{0}, z_{1}, \ldots\right) \operatorname{sen}\left(\omega \tau_{0}\right)+s_{1}\left(z_{0}, z_{1}, \ldots\right) \cos \left(\omega \tau_{0}\right)$

$c_{1}=p_{1} \operatorname{sen}\left(\omega \tau_{0}\right)+q_{1} \cos \left(\omega \tau_{0}\right)$,

sendo $\omega$ uma freqüência natural adimensionalizada. Decorre que:

$$
\begin{aligned}
& \frac{1}{4} \Delta_{0}{ }^{4} r_{1}+c_{0}{ }^{4}\left(H-\omega^{2}\right) r_{1}=-c_{0}{ }^{3} p_{1}\left[\omega^{2}\left(1+z_{0}\right)\left(\Delta_{0} \hat{u}\right)+4(1+H \hat{u})\right] \\
& \frac{1}{4} \Delta_{0}{ }^{4} s_{1}+c_{0}{ }^{4}\left(H-\omega^{2}\right) s_{1}=-c_{0}{ }^{3} q_{1}\left[\omega^{2}\left(1+z_{0}\right)\left(\Delta_{0} \hat{u}\right)+4(1+H \hat{u})\right]
\end{aligned}
$$

Pode-se mostrar em Mazzilli e Lenci [21], que não há soluções para $\omega>1$, de sorte que doravante se considerará apenas o caso de $\omega<1$.

A solução de (24) e (25), considerando (13), para $-1<z_{0}<0$, é:

$$
\begin{aligned}
r_{1 e}\left(z_{0}\right)=R_{e 1} & \operatorname{sen}\left(\alpha_{e} \sqrt{2} z_{0}\right)+R_{e 2} \cos \left(\alpha_{e} \sqrt{2} z_{0}\right)+R_{e 3} e^{\alpha_{e} \sqrt{2} z_{0}}+R_{e 4} e^{-\alpha_{e} \sqrt{2} z_{0}} \\
& +p_{1}\left[-\left(1+c_{0}\right)+\left(2 c_{0}{ }^{2}-c_{0}-1\right) z_{0}+{c_{0}}^{2}\left(3-c_{0}\right) z_{0}{ }^{2}\right. \\
& \left.-c_{0}{ }^{2}\left(\frac{5 c_{0}}{3}-1\right) z_{0}{ }^{3}-\frac{2}{3} c_{0}{ }^{3} z_{0}{ }^{4}\right] \\
s_{1 e}\left(z_{0}\right)=S_{e 1} & \operatorname{sen}\left(\alpha_{e} \sqrt{2} z_{0}\right)+S_{e 2} \cos \left(\alpha_{e} \sqrt{2} z_{0}\right)+S_{e 3} e^{\alpha_{e} \sqrt{2} z_{0}}+S_{e 4} e^{-\alpha_{e} \sqrt{2} z_{0}} \\
& +q_{1}\left[-\left(1+c_{0}\right)+\left(2 c_{0}{ }^{2}-c_{0}-1\right) z_{0}+c_{0}{ }^{2}\left(3-c_{0}\right) z_{0}{ }^{2}\right. \\
& \left.-c_{0}{ }^{2}\left(\frac{5 c_{0}}{3}-1\right) z_{0}{ }^{3}-\frac{2}{3} c_{0}{ }^{3} z_{0}{ }^{4}\right]
\end{aligned}
$$

com $\alpha_{e}=c_{0} \sqrt{\omega}$ e 8 coeficientes $\left(R_{e i} e S_{e i}, i=1 a 4\right)$ a determinar. Lembre-se que, neste trecho, há reflexão e refração de onda no TDP, por conta das condições de contato unilateral. 
Já a solução de (24) e (25) para $z_{0}>0$ é:

$$
\begin{aligned}
r_{1 d}\left(z_{0}\right)=e^{\alpha_{d} z_{0}} & {\left[R_{d 1} \operatorname{sen}\left(\alpha_{d} z_{0}\right)+R_{d 2} \cos \left(\alpha_{d} z_{0}\right)\right] } \\
& +e^{-\alpha_{d} z_{0}}\left[R_{d 3} \operatorname{sen}\left(\alpha_{d} z_{0}\right)+R_{d 4} \cos \left(\alpha_{d} z_{0}\right)\right] \\
& +p_{1} e^{-c_{0} z_{0}}\left[-\left(1+c_{0}\right) \cos \left(c_{0} z_{0}\right)-\left(1-c_{0}\right) \operatorname{sen}\left(c_{0} z_{0}\right)\right. \\
& \left.-\left(1+c_{0}\right) z_{0} \cos \left(c_{0} z_{0}\right)-\left(1-c_{0}\right) z_{0} \operatorname{sen}\left(c_{0} z_{0}\right)\right], \\
s_{1 d}\left(z_{0}\right)=e^{\alpha_{d} z_{0}} & {\left[S_{d 1} \operatorname{sen}\left(\alpha_{d} z_{0}\right)+S_{d 2} \cos \left(\alpha_{d} z_{0}\right)\right] } \\
& +e^{-\alpha_{d} z_{0}}\left[S_{d 3} \operatorname{sen}\left(\alpha_{d} z_{0}\right)+S_{d 4} \cos \left(\alpha_{d} z_{0}\right)\right] \\
& +q_{1} e^{-c_{0} z_{0}}\left[-\left(1+c_{0}\right) \cos \left(c_{0} z_{0}\right)-\left(1-c_{0}\right) \operatorname{sen}\left(c_{0} z_{0}\right)\right. \\
& \left.-\left(1+c_{0}\right) z_{0} \cos \left(c_{0} z_{0}\right)-\left(1-c_{0}\right) z_{0} \operatorname{sen}\left(c_{0} z_{0}\right)\right],
\end{aligned}
$$

com $\alpha_{d}=c_{0} \sqrt[4]{1-\omega^{2}}$ e 4 coeficientes $\left(R_{d i}\right.$ e $S_{d i}, i=3$ e 4$)$ a determinar, uma vez que, neste trecho, despreza-se a onda refletida em $+\infty$, que implica $R_{d 1}=R_{d 2}=$ $S_{d 1}=S_{d 2}=0$.

Aplicam-se, agora, às equações (28) e (29) acima, as condições de contorno da solução dinâmica (17). Cada uma das condições descritas em (17), por sua vez, gera duas equações, uma para $r_{1}$ e outra para $s_{1}$, fazendo com que os 12 coeficientes a serem determinados, e mais $p_{1}$ e $q_{1}$, estejam num sistema com 14 equações:

$$
\begin{aligned}
& -R_{e 1} \operatorname{sen}\left(\alpha_{e} \sqrt{2}\right)+R_{e 2} \cos \left(\alpha_{e} \sqrt{2}\right)+R_{e 3} e^{-\alpha_{e} \sqrt{2}}+R_{e 4} e^{\alpha_{e} \sqrt{2}}=0 \\
& -S_{e 1} \operatorname{sen}\left(\alpha_{e} \sqrt{2}\right)+S_{e 2} \cos \left(\alpha_{e} \sqrt{2}\right)+S_{e 3} e^{-\alpha_{e} \sqrt{2}}+S_{e 4} e^{\alpha_{e} \sqrt{2}}=0 \\
& R_{e 1} \operatorname{sen}\left(\alpha_{e} \sqrt{2}\right)-R_{e 2} \cos \left(\alpha_{e} \sqrt{2}\right)+R_{e 3} e^{-\alpha_{e} \sqrt{2}}+R_{e 4} e^{\alpha_{e} \sqrt{2}}=0 \\
& S_{e 1} \operatorname{sen}\left(\alpha_{e} \sqrt{2}\right)-S_{e 2} \cos \left(\alpha_{e} \sqrt{2}\right)+S_{e 3} e^{-\alpha_{e} \sqrt{2}}+S_{e 4} e^{\alpha_{e} \sqrt{2}}=0 \\
& R_{e 2}+R_{e 3}+R_{e 4}=\left(1+c_{0}\right) p_{1} \\
& S_{e 2}+S_{e 3}+S_{e 4}=\left(1+c_{0}\right) q_{1} \\
& R_{d 4}=\left(1+c_{0}\right) p_{1} \\
& S_{d 4}=\left(1+c_{0}\right) q_{1} \\
& R_{e 1}+R_{e 3}-R_{e 4}=\frac{\sqrt{2}}{2} \eta\left[R_{d 3}-\left(1+c_{0}\right) p_{1}\right]
\end{aligned}
$$




$$
\begin{aligned}
& S_{e 1}+S_{e 3}-S_{e 4}=\frac{\sqrt{2}}{2} \eta\left[S_{d 3}-\left(1+c_{0}\right) q_{1}\right] \\
& -R_{e 2}+R_{e 3}+R_{e 4}=-\eta^{2} R_{d 3} \\
& -S_{e 2}+S_{e 3}+S_{e 4}=-\eta^{2} S_{d 3} \\
& -R_{e 1}+R_{e 3}-R_{e 4}=\frac{\sqrt{2}}{2} \eta^{3}\left[R_{d 3}+\left(1+c_{0}\right) p_{1}\right] \\
& -S_{e 1}+S_{e 3}-S_{e 4}=\frac{\sqrt{2}}{2} \eta^{3}\left[S_{d 3}+\left(1+c_{0}\right) q_{1}\right]
\end{aligned}
$$

onde:

$$
\eta=\frac{\alpha_{d}}{\alpha_{e}}=\sqrt[4]{\frac{1-\omega^{2}}{\omega^{2}}}
$$

Todos os coeficientes $R_{e i}, i=1 a 4$ podem ser expressos em termos de $R_{d 3}$ e $p_{1}$ (analogamente $S_{d 3}$ e $q_{1}$, para $S_{e i}, i=1 a 4$ ). Em especial, resulta que:

$R_{e i}=A_{i} R_{d 3}+B_{i} p_{1}$, tal que $i=1 a 4$

$S_{e i}=A_{i} S_{d 3}+B_{i} q_{1}$, tal que $i=1 a 4$,

com os coeficientes $A_{i}$ e $B_{i}$ sendo:

$$
\begin{aligned}
& A_{1}=\frac{\sqrt{2}}{4} \eta\left(1-\eta^{2}\right) \\
& B_{1}=-\frac{\sqrt{2}}{4} \eta\left(1+\eta^{2}\right)\left(1+c_{0}\right) \\
& A_{2}=\frac{\eta^{2}}{2} \\
& B_{2}=\frac{\left(1+c_{0}\right)}{2} \\
& A_{3}=\frac{\eta}{4}\left(\frac{\sqrt{2}}{2}+\frac{\sqrt{2}}{2} \eta^{2}-\eta\right) \\
& B_{3}=\frac{1}{4}\left(1-\frac{\sqrt{2}}{2} \eta+\frac{\sqrt{2}}{2} \eta^{3}\right)\left(1+c_{0}\right)
\end{aligned}
$$




$$
\begin{aligned}
& A_{4}=-\frac{\eta}{4}\left(\frac{\sqrt{2}}{2}+\frac{\sqrt{2}}{2} \eta^{2}+\eta\right) \\
& B_{4}=\frac{1}{4}\left(1+\frac{\sqrt{2}}{2} \eta-\frac{\sqrt{2}}{2} \eta^{3}\right)\left(1+c_{0}\right) .
\end{aligned}
$$

Considerando (45) em (30) e (32), ou (46) em (31) e (33) chega-se a um sistema homogêneo:

$\left[\begin{array}{ll}K_{11} & K_{12} \\ K_{21} & K_{22}\end{array}\right]\left\{\begin{array}{c}R_{3 d} \\ p_{1}\end{array}\right\}=\left\{\begin{array}{l}0 \\ 0\end{array}\right\}$ ou $\left[\begin{array}{ll}K_{11} & K_{12} \\ K_{21} & K_{22}\end{array}\right]\left\{\begin{array}{c}S_{3 d} \\ q_{1}\end{array}\right\}=\left\{\begin{array}{l}0 \\ 0\end{array}\right\}$

onde:

$$
\begin{aligned}
& K_{11}=-A_{1} \operatorname{sen}\left(\alpha_{e} \sqrt{2}\right)+A_{2} \cos \left(\alpha_{e} \sqrt{2}\right)+A_{3} e^{-\alpha_{e} \sqrt{2}}+A_{4} e^{\alpha_{e} \sqrt{2}} \\
& K_{12}=-B_{1} \operatorname{sen}\left(\alpha_{e} \sqrt{2}\right)+B_{2} \cos \left(\alpha_{e} \sqrt{2}\right)+B_{3} e^{-\alpha_{e} \sqrt{2}}+B_{4} e^{\alpha_{e} \sqrt{2}} \\
& K_{21}=A_{1} \operatorname{sen}\left(\alpha_{e} \sqrt{2}\right)-A_{2} \cos \left(\alpha_{e} \sqrt{2}\right)+A_{3} e^{-\alpha_{e} \sqrt{2}}+A_{4} e^{\alpha_{e} \sqrt{2}} \\
& K_{22}=B_{1} \operatorname{sen}\left(\alpha_{e} \sqrt{2}\right)-B_{2} \cos \left(\alpha_{e} \sqrt{2}\right)+B_{3} e^{-\alpha_{e} \sqrt{2}}+B_{4} e^{\alpha_{e} \sqrt{2}} .
\end{aligned}
$$

A solução não trivial só é possível quando $|K|=0$, que é a equação característica da análise modal do problema com contato unilateral. Tal equação pode ser escrita em termos da variável $\eta$, (vide 44), isto é, $f\left(\eta, c_{0}\right)=0$, sendo que, para cada valor de $\eta$ encontrado associa-se a freqüência natural normalizada correspondente:

$$
\omega=\frac{1}{\sqrt{1+\eta^{4}}} .
$$

A Fig. 4 permite visualizar que para maiores valores de $c_{0}$ a superfície $f\left(\eta, c_{0}\right)$ cruza o plano $f\left(\eta, c_{0}\right)=0$ um maior número de vezes: 


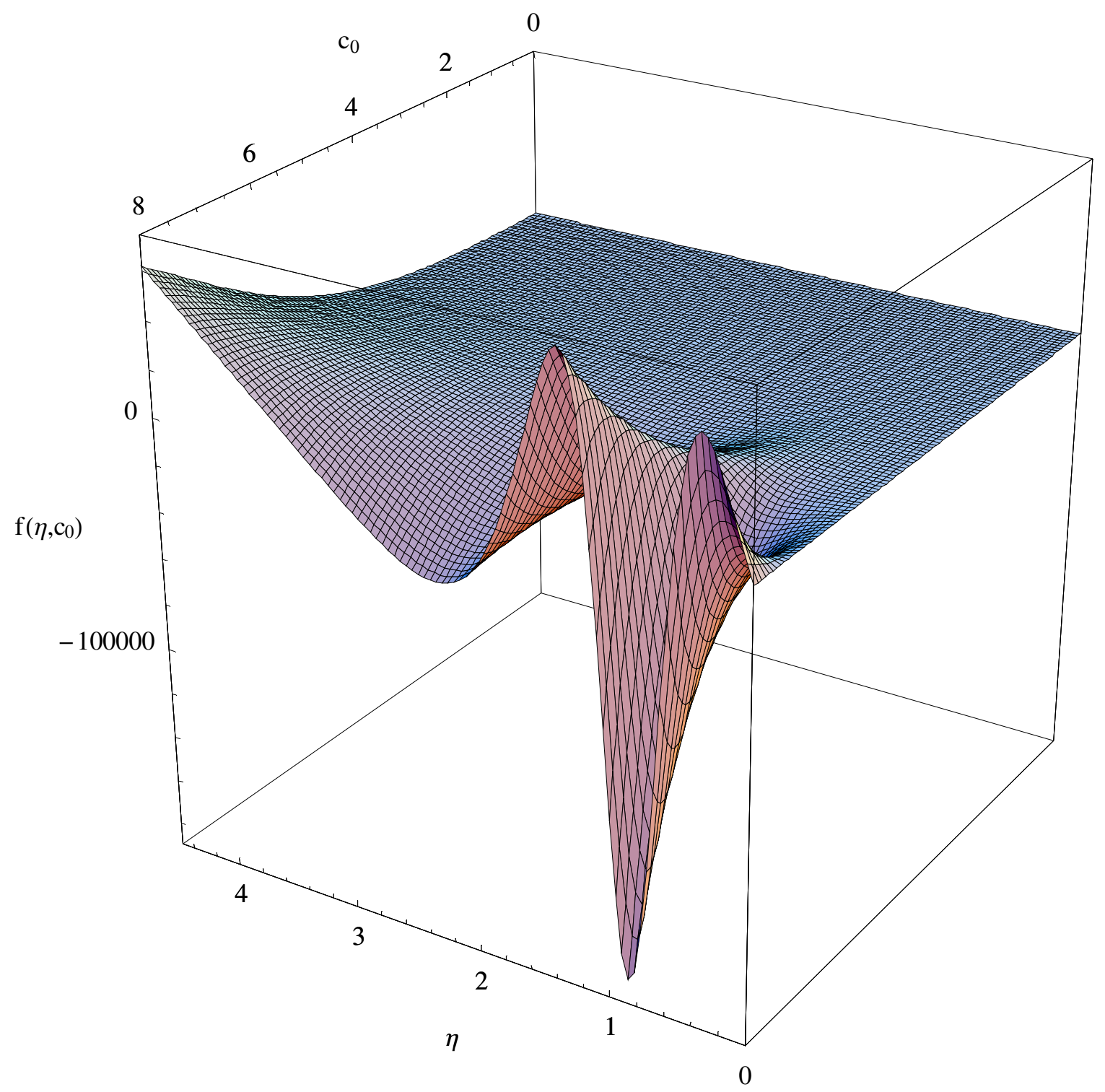

Figura 7: Superfície $f\left(\eta, c_{0}\right)$

Alguns resultados gráficos são mostrados a seguir para a função $f(\eta)$ variando-se os valores de $c_{0}$. Assim, a partir da superfície ilustrada na Fig. 7 , são feitas projeções no plano $f(\eta) \times \eta$ com diferentes valores de $c_{0}$, de interesse para o estudo, a partir das quais se pode obter os modos de vibração para o modelo em questão: 


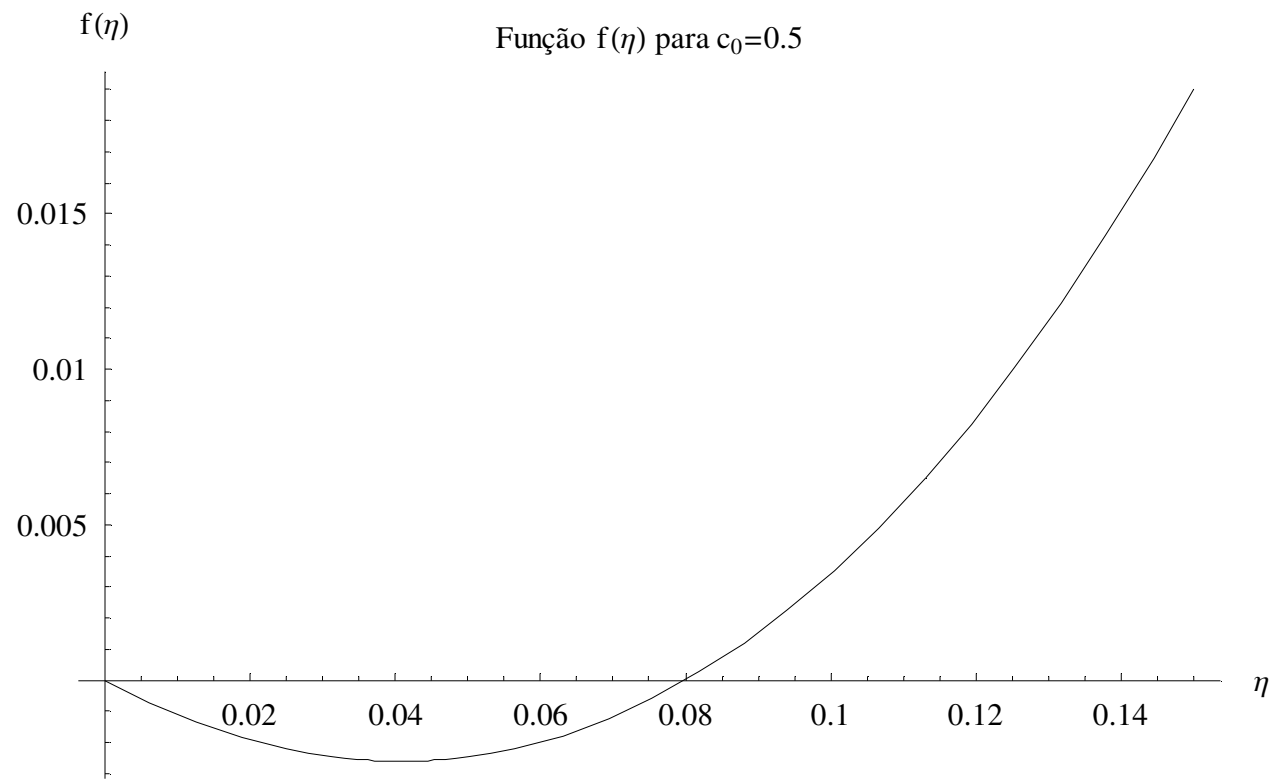

Figura 8: Uma solução (modos de vibração) correspondentes a $f(\eta)=0$ para $c_{0}=0.5$

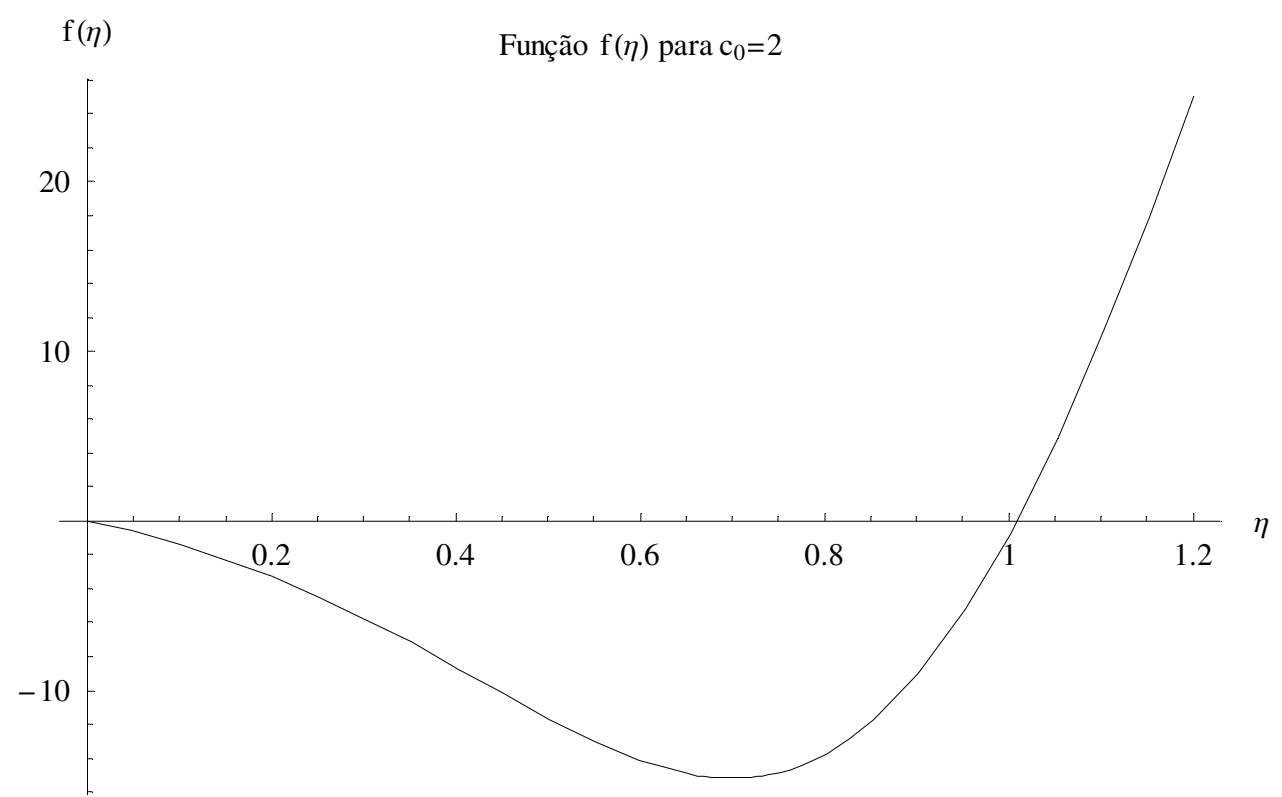

Figura 9: Uma solução (modos de vibração) correspondentes a $f(\eta)=0$ para $c_{0}=2$ 


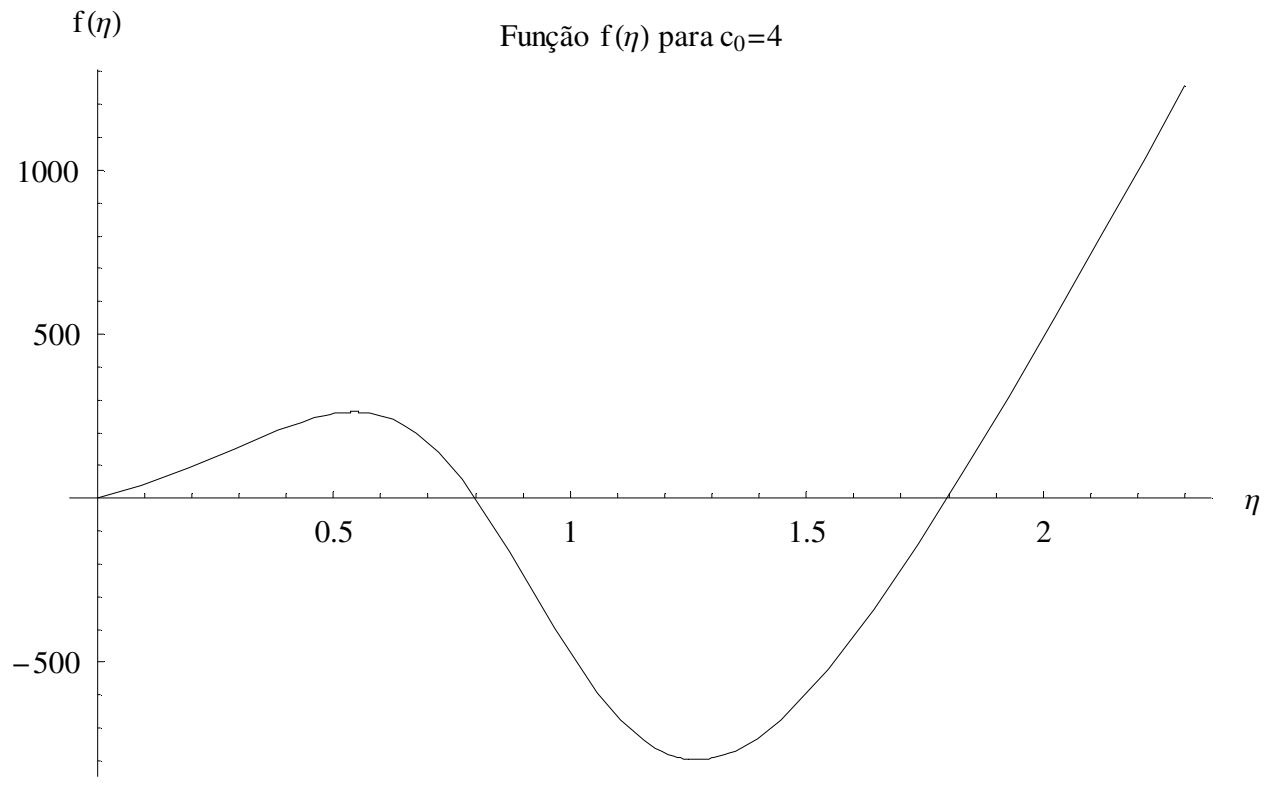

Figura 10: Duas soluções (modos de vibração) correspondentes a $f(\eta)=0$ para $c_{0}=4$

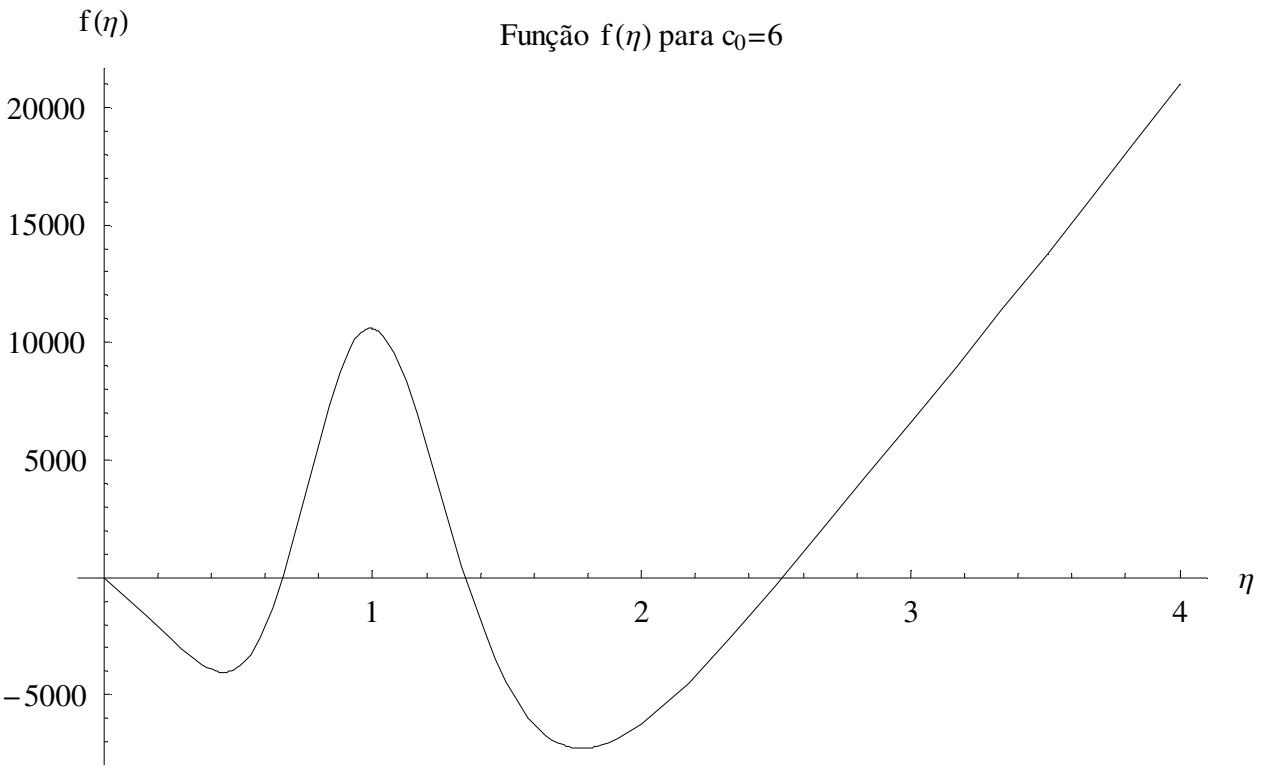

Figura 11: Três soluções (modos de vibração) correspondentes a $f(\eta)=0$ para $c_{0}=6$ 


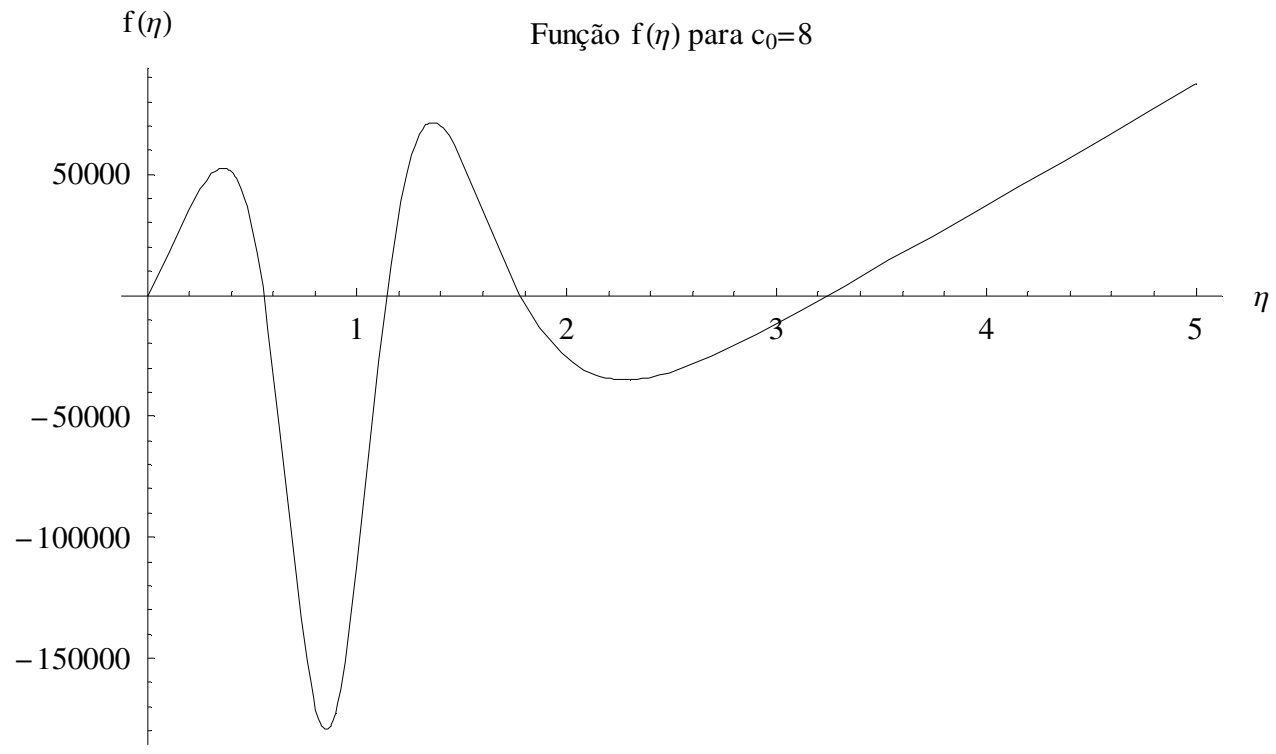

Figura 12: Quatro soluções (modos de vibração) correspondentes a $f(\eta)=0$ para $c_{0}=8$

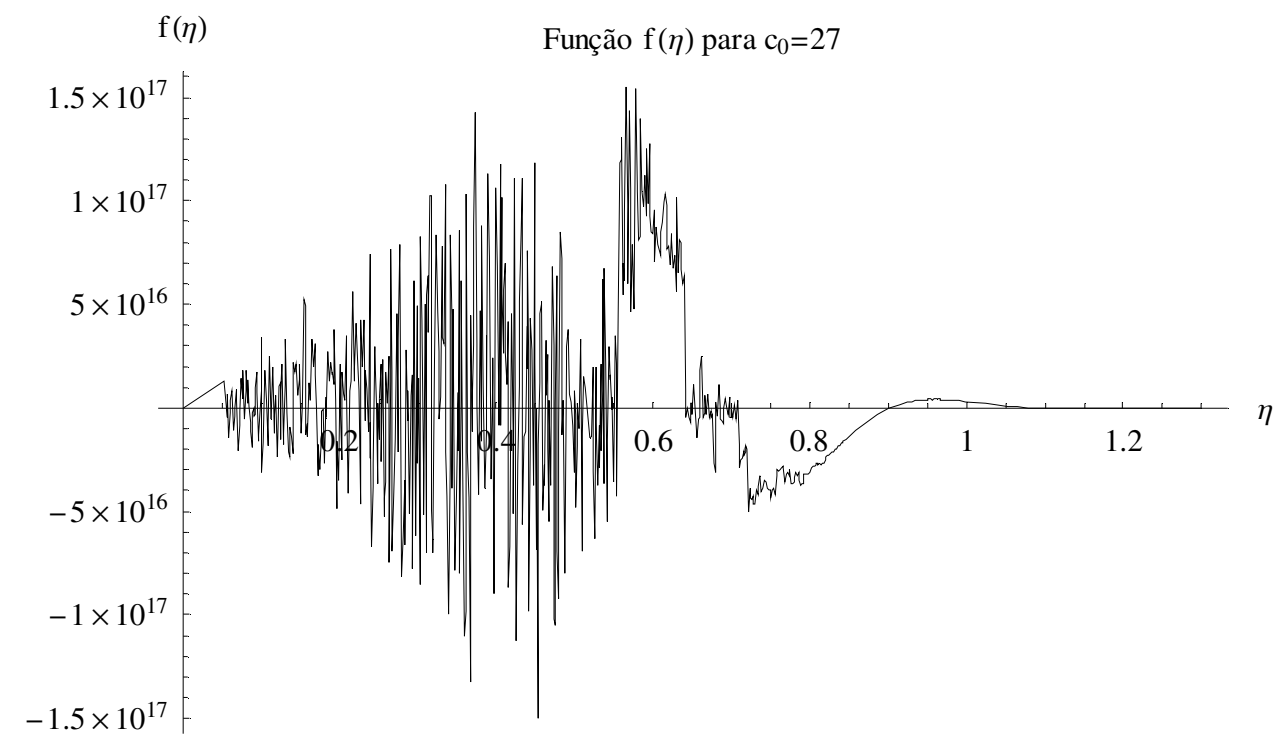

Figura 13: Múltilplas soluções (modos de vibração) correspondentes a $f(\eta)=0$ para $c_{0}=27$ 
Evidentemente, para limitar os deslocamentos transversais $w_{0}\left(\hat{u}_{0}\right)$, impõese também limitar $x_{c, 0}\left(c_{0}\right)$, de forma a não violar as hipóteses da teoria elementar de barras.

A tabela a seguir ilustra as relações entre $c_{0}$ (posição do $T D P$ ), $\eta$, e $\omega$ (freqüência natural normalizada) encontradas:

\begin{tabular}{|c|c|c|}
\hline \multirow{2}{*}{$\boldsymbol{c}_{\mathbf{0}}$} & $\boldsymbol{\eta}=\sqrt[4]{\frac{\mathbf{1 - \boldsymbol { \omega } ^ { 2 }}}{\boldsymbol{\omega}^{2}}}$ & $\boldsymbol{\omega}$ \\
\hline \multirow{2}{*}{8} & 3.24573 & 0.094499 \\
\cline { 2 - 3 } & 1.77947 & 0.301145 \\
\cline { 2 - 3 } & 1.14196 & 0.608512 \\
\cline { 2 - 3 } & 0.555529 & 0.955532 \\
\hline \multirow{2}{*}{6} & 2.52496 & 0.154958 \\
\cline { 2 - 3 } & 1.34546 & 0.483535 \\
\cline { 2 - 3 } & 0.667872 & 0.913266 \\
\hline \multirow{2}{*}{4} & 1.79746 & 0.295675 \\
\hline \multirow{2}{*}{4} & 0.800533 & 0.700651 \\
\hline \multirow{2}{*}{2} & 1.00913 & 0.99998 \\
\hline
\end{tabular}

Tabela 1: Freqüências modais adimensionais $(\omega)$ em função da posição do $\operatorname{TDP}\left(c_{0}\right)$

Note-se que a particularidade de número finito de modos de vibração, para um determinado valor de $c_{0}$, está associada à condição de $c_{0}$ ser menor do que 25, aproximadamente. 


\subsection{Determinação das relações modais}

Tem-se, aqui, como objetivo, a determinação das relações modais, isto é, as relações entre os deslocamentos dinâmicos $\delta(z, \tau)$ e a variável modal a ser escolhida $U$, recuperando, parcialmente, as informações topológicas do método das variedades invariantes (MVI). As relações modais desempenham papel central na obtenção dos modelos de ordem reduzida, como se verá oportunamente.

Recorde-se, do sistema descrito em (55), que:

$K_{11} R_{3 d}+K_{12} p_{1}=0$,

de onde chegamos a:

$R_{d 3}=\beta^{*} p_{1}$,

onde:

$\beta^{*}=-\frac{K_{12}}{K_{11}}$.

Portanto, de (45) e (46) decorrem:

$R_{i e}=A_{i} R_{3 d}+B_{i} p_{1}=\left(A_{i} \beta^{*}+B_{i}\right) p_{1}=C_{i} p_{1}$
$S_{i e}=A_{i} R_{3 d}+B_{i} q_{1}=\left(A_{i} \beta^{*}+B_{i}\right) q_{1}=C_{i} q_{1}$,

de onde, a partir de (18): 


$$
\begin{aligned}
\delta_{e}=\varepsilon\left[C_{1} \operatorname{sen}\right. & \left(\alpha_{e} \sqrt{2} z_{0}\right)+C_{2} \cos \left(\alpha_{e} \sqrt{2} z_{0}\right)+C_{3} e^{\alpha_{e} \sqrt{2} z_{0}}+C_{4} e^{-\alpha_{e} \sqrt{2} z_{0}}-\left(1+c_{0}\right) \\
& +\left(2 c_{0}{ }^{2}-c_{0}-1\right) z_{0}+c_{0}{ }^{2}\left(3-c_{0}\right) z_{0}{ }^{2}-c_{0}{ }^{2}\left(\frac{5}{3} c_{0}-1\right) z_{0}{ }^{3} \\
& \left.-\frac{2}{3} c_{0}{ }^{3} z_{0}{ }^{4}\right]\left[p_{1} \operatorname{sen}\left(\omega \tau_{0}\right)+q_{1} \cos \left(\omega \tau_{0}\right)\right]
\end{aligned}
$$

para $-1<z_{0}<0$, e

$$
\begin{aligned}
\delta_{d}=\varepsilon\left\{e^{-\alpha_{d} z_{0}}\right. & {\left[\beta^{*} \operatorname{sen}\left(\alpha_{d} z_{0}\right)+\left(1+c_{0}\right) \cos \left(\alpha_{d} z_{0}\right)\right]+e^{-c_{0} z_{0}}\left[-\left(1+c_{0}\right) \cos \left(c_{0} z_{0}\right)\right.} \\
& -\left(1-c_{0}\right) \operatorname{sen}\left(c_{0} z_{0}\right)-\left(1+c_{0}\right) z_{0} \cos \left(c_{0} z_{0}\right) \\
& \left.\left.-\left(1-c_{0}\right) z_{0} \operatorname{sen}\left(c_{0} z_{0}\right)\right]\right\}\left[p_{1} \operatorname{sen}\left(\omega \tau_{0}\right)+q_{1} \cos \left(\omega \tau_{0}\right)\right],
\end{aligned}
$$

para $z_{0}>0$.

A escolha que aqui se faz para a variável modal $U$, é o deslocamento horizontal adimensional do TDP:

$U=\varepsilon\left[p_{1} \operatorname{sen}\left(\omega \tau_{0}\right)+q_{1} \cos \left(\omega \tau_{0}\right)\right]$

decorrendo $p_{1}$ e $q_{1}$ das condições iniciais do problema. Por derivação em relação a $\tau_{0}$, obtém-se a expressão da velocidade horizontal adimensional do TDP:

$V=\dot{U}=\varepsilon \omega\left[p_{1} \cos \left(\omega \tau_{0}\right)-q_{1} \operatorname{sen}\left(\omega \tau_{0}\right)\right]$

Assim, reescrevem-se as equações (68) e (69) de maneira a obter o seguinte sistema:

$\left[\begin{array}{cc}p_{1} & q_{1} \\ -q_{1} & p_{1}\end{array}\right]\left\{\begin{array}{c}\operatorname{sen}\left(\omega \tau_{0}\right) \\ \cos \left(\omega \tau_{0}\right)\end{array}\right\}=\left\{\begin{array}{c}\frac{U}{\varepsilon} \\ \frac{V}{\varepsilon \omega}\end{array}\right\}$

A partir daí obtemos as expressões: 
$\operatorname{sen}\left(\omega \tau_{0}\right)=\frac{\left|\begin{array}{cc}\frac{U}{\varepsilon} & q_{1} \\ \frac{V}{\varepsilon \omega} & p_{1}\end{array}\right|}{p_{1}{ }^{2}+q_{1}{ }^{2}}=\frac{\frac{U}{\varepsilon} p_{1}-\frac{V}{\varepsilon \omega} q_{1}}{p_{1}{ }^{2}+q_{1}{ }^{2}}=\frac{U p_{1}-\frac{V}{\omega} q_{1}}{\varepsilon\left(p_{1}{ }^{2}+q_{1}{ }^{2}\right)}$

e

$$
\cos \left(\omega \tau_{0}\right)=\frac{\left|\begin{array}{cc}
p_{1} & \frac{U}{\varepsilon} \\
-q_{1} & \frac{V}{\varepsilon \omega}
\end{array}\right|}{p_{1}^{2}+q_{1}^{2}}=\frac{\frac{U}{\varepsilon} q_{1}+\frac{V}{\varepsilon \omega} p_{1}}{p_{1}^{2}+q_{1}^{2}}=\frac{U q_{1}+\frac{V}{\omega} p_{1}}{\varepsilon\left(p_{1}^{2}+q_{1}^{2}\right)}
$$

Substituindo (71) e (72) em (66) e (67), obtemos as expressões para as soluções dinâmicas em função da variável modal $U$, à esquerda e à direita do TDP:

$$
\begin{aligned}
\delta_{e}=\left[C_{1} \operatorname{sen}(\right. & \left.\alpha_{e} \sqrt{2} z_{0}\right)+C_{2} \cos \left(\alpha_{e} \sqrt{2} z_{0}\right)+C_{3} e^{\alpha_{e} \sqrt{2} z_{0}}+C_{4} e^{-\alpha_{e} \sqrt{2} z_{0}}-\left(1+c_{0}\right) \\
& +\left(2 c_{0}{ }^{2}-c_{0}-1\right) z_{0}+c_{0}{ }^{2}\left(3-c_{0}\right) z_{0}{ }^{2}-c_{0}{ }^{2}\left(\frac{5}{3} c_{0}-1\right) z_{0}{ }^{3} \\
& \left.-\frac{2}{3} c_{0}{ }^{3} z_{0}{ }^{4}\right] U
\end{aligned}
$$

e

$$
\begin{aligned}
\delta_{d}=\left\{e^{-\alpha_{d} z_{0}}[\right. & \left.\beta^{*} \operatorname{sen}\left(\alpha_{d} z_{0}\right)+\left(1+c_{0}\right) \cos \left(\alpha_{d} z_{0}\right)\right]+e^{-c_{0} z_{0}}\left[-\left(1+c_{0}\right) \cos \left(c_{0} z_{0}\right)\right. \\
& -\left(1-c_{0}\right) \operatorname{sen}\left(c_{0} z_{0}\right)-\left(1+c_{0}\right) z_{0} \cos \left(c_{0} z_{0}\right) \\
& \left.\left.-\left(1-c_{0}\right) z_{0} \operatorname{sen}\left(c_{0} z_{0}\right)\right]\right\} U
\end{aligned}
$$

Definem-se então as funções: 


$$
\begin{gathered}
\zeta_{e}\left(z_{0}\right)=\left[C_{1} \operatorname{sen}\left(\alpha_{e} \sqrt{2} z_{0}\right)+C_{2} \cos \left(\alpha_{e} \sqrt{2} z_{0}\right)+C_{3} e^{\alpha_{e} \sqrt{2} z_{0}}+C_{4} e^{-\alpha_{e} \sqrt{2} z_{0}}-\left(1+c_{0}\right)\right. \\
+\left(2 c_{0}{ }^{2}-c_{0}-1\right) z_{0}+c_{0}{ }^{2}\left(3-c_{0}\right) z_{0}{ }^{2}-c_{0}{ }^{2}\left(\frac{5}{3} c_{0}-1\right) z_{0}{ }^{3} \\
\left.-\frac{2}{3} c_{0}{ }^{3} z_{0}{ }^{4}\right]
\end{gathered}
$$

e

$$
\begin{aligned}
\zeta_{d}\left(z_{0}\right)=e^{-\alpha_{d} z_{0}} & {\left[\beta^{*} \operatorname{sen}\left(\alpha_{d} z_{0}\right)+\left(1+c_{0}\right) \cos \left(\alpha_{d} z_{0}\right)\right] } \\
+ & e^{-c_{0} z_{0}}\left[-\left(1+c_{0}\right) \cos \left(c_{0} z_{0}\right)-\left(1-c_{0}\right) \operatorname{sen}\left(c_{0} z_{0}\right)\right. \\
& \left.-\left(1+c_{0}\right) z_{0} \cos \left(c_{0} z_{0}\right)-\left(1-c_{0}\right) z_{0} \operatorname{sen}\left(c_{0} z_{0}\right)\right]
\end{aligned}
$$

e reescrevemos as relações (77) e (78) da seguinte forma:

$$
\delta_{e}=\zeta_{e}\left(z_{0}\right) U
$$

e

$\delta_{d}=\zeta_{d}\left(z_{0}\right) U$

A partir de (64) e (65), determinamos os coeficientes para $R_{e i}, i=1 a 4$, e analogamente para $S_{e i}, i=1 a 4$, concluindo a recuperação das relações modais e caracterizando topologicamente a variedade em que o movimento do oscilador modal transcorre. 
3. Capítulo 3: Modos não-lineares de vibração e modelo de ordem reduzida de viga esbelta semi-infinita com contato unilateral em apoio elástico sob flexão composta

\subsection{Formulação do problema com consideração do efeito de tração}

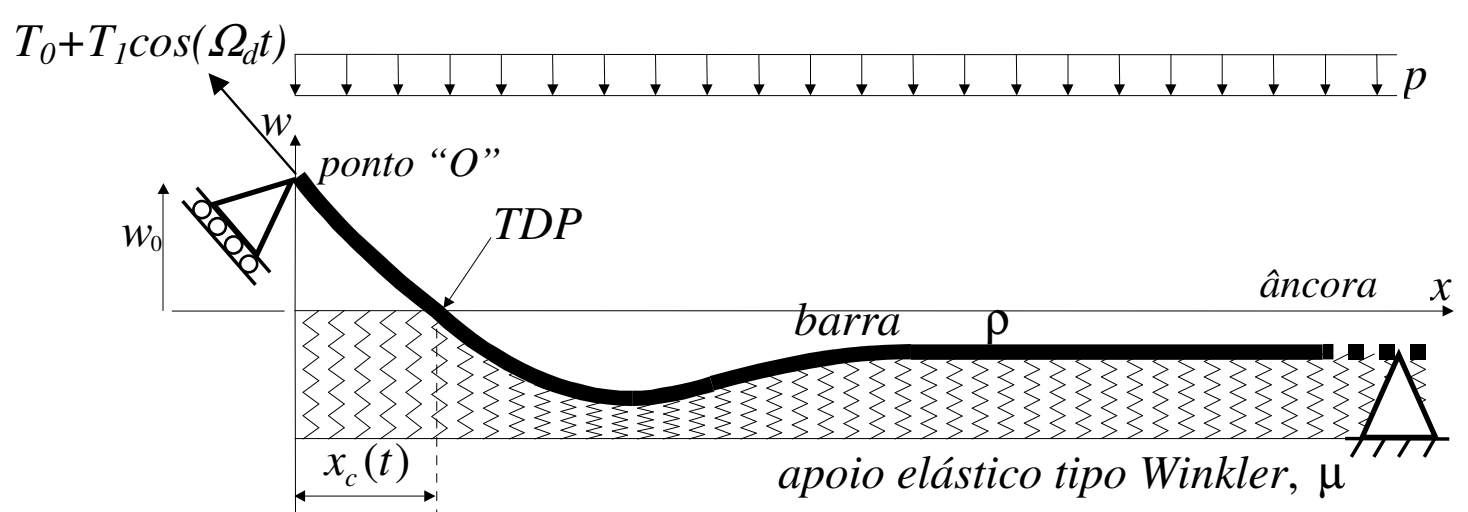

Figura 14: Viga esbelta semi-infinita com contato unilateral em apoio elástico sob flexo-tração estática e dinâmica

A equação para o modelo estudado de barra semi-infinita inicialmente jazendo sobre um meio elástico horizontal com coeficiente de rigidez $\mu$, tendo massa por unidade de comprimento $\rho$ (incluindo a massa adicional), sob carregamento uniformemente distribuído $p$, e sujeita à flexão composta decorrente da imposição de um deslocamento vertical $w_{0}$ e à tração imposta na extremidade suspensa $T_{0}+T_{1} \cos \left(\Omega_{d} t\right)$, onde $\Omega_{d}$ corresponde à freqüência dimensional da tração imposta, conforme se apresenta na Fig. 14, pode ser expressa da seguinte forma:

$E I \frac{\partial^{4} w}{\partial x^{4}}+\rho \frac{\partial^{2} w}{\partial t^{2}}-T \frac{\partial^{2} w}{\partial x^{2}}-\frac{\partial T}{\partial x} \frac{\partial w}{\partial x}+p=0$

para $0<x<x_{c}$, e

$E I \frac{\partial^{4} w}{\partial x^{4}}+\mu w+\rho \frac{\partial^{2} w}{\partial t^{2}}-T \frac{\partial^{2} w}{\partial x^{2}}-\frac{\partial T}{\partial x} \frac{\partial w}{\partial x}+p=0$ 
para $x>x_{c}$, sendo $x_{c}$ a posição estática do ponto de contato, ou simplesmente TDP.

Note-se que as não-linearidades geométricas da teoria de barras foram ignoradas, conforme se depreende da linearização da curvatura, $\left(\partial^{4} w / \partial x^{4}\right)$ implícita em (79) e (80), uma vez que as não-linearidades oriundas do contato são muito mais fortes, analogamente ao que foi considerado no capítulo 2.

Portanto, (79) e (80) traduzem o equilíbrio dinâmico na direção vertical do modelo da teoria elementar de barras com apoio unilateral elástico, ao qual se introduz a força aparente de inércia, segundo o princípio de D’Alambert, semelhante ao que foi adotado no capítulo 2.

A distinção que existe entre (79)-(80), e (1)-(2) é, evidentemente, a incorporação ao modelo descrito no capítulo 2 da variação da projeção vertical da tração:

$\frac{\partial}{\partial x}\left(T \frac{\partial w}{\partial x}\right)=-T \frac{\partial^{2} w}{\partial x^{2}}-\frac{\partial T}{\partial x} \frac{\partial w}{\partial x}$

Com o intuito de escrever as equações acima na forma adimensional, definem-se as seguintes relações:

$y=\alpha x, \quad v=\frac{\mu}{p} w, \quad \tau=\beta t, \quad \alpha=\sqrt[4]{\frac{\mu}{4 E I}}, \quad \beta=\sqrt{\frac{\mu}{\rho}} \quad e \quad \gamma=\frac{T}{2 \sqrt{\mu E I}}$

Aplicando-se as relações acima, chegamos às equações adimensionais que descrevem o problema:

$\frac{1}{4} \frac{\partial^{4} v}{\partial y^{4}}+\frac{\partial^{2} v}{\partial \tau^{2}}-\gamma \frac{\partial^{2} v}{\partial y^{2}}-\frac{\partial \gamma}{\partial y} \frac{\partial v}{\partial y}+1=0$

para $0<y<c(\tau), c(\tau)=\alpha x_{c}(t), \mathrm{e}$ 
$\frac{1}{4} \frac{\partial^{4} v}{\partial y^{4}}+\frac{\partial^{2} v}{\partial \tau^{2}}+v-\gamma \frac{\partial^{2} v}{\partial y^{2}}-\frac{\partial \gamma}{\partial y} \frac{\partial v}{\partial y}+1=0$

para $y>c(\tau)$.

Neste contexto, a posição do (TDP) fica definida como sendo aquela em que $v[c(\tau), \tau]=0$.

Com o intuito de transformar o problema acima de condições de contorno móveis em um de condições de contorno fixas, faz-se, mais uma vez, uso da mesma transformação utilizada no capítulo 2:

$z=\frac{y}{c(\tau)}-1 \Rightarrow y=(z+1) c(\tau)$

e uma nova função é definida:

$u(z, \tau)=v(y, \tau)$

Analogamente ao que foi desenvolvido na seção 2.1, será adotada no prosseguimento deste trabalho a notação ("), "linha”, para representar as derivadas espaciais em relação à $z$, e a notação (·), "ponto", para representar as derivadas temporais em relação à $\tau$.

Assim, reescrevem-se as equações (83) e (84), com a consideração da equação (85), e com ajuda da função de Heavyside $(H(z))$ (deduções constam no anexo 7.1) chega-se a:

$$
\begin{gathered}
\frac{1}{4} u^{I V}-2 \dot{u}^{\prime} \dot{c}(\mathrm{z}+1) c^{3}+u^{\prime \prime} c^{2}\left[\dot{c}^{2}(1+z)^{2}-\gamma\right]+u^{\prime}\left[(z+1)\left(2 \dot{c}^{2}-\ddot{c} c\right)-\gamma^{\prime} c\right] c^{2}+c^{4} \ddot{u} \\
+H c^{4} u+c^{4}=0,
\end{gathered}
$$

sendo: 


$$
\begin{aligned}
& H(z)=0, \quad-1<z<0 \\
& H(z)=1, \quad z>0 .
\end{aligned}
$$

Como a equação (87) restringe-se ao equilíbrio dinâmico na vertical, adotar-se-á a hipótese de variação da tração ao longo da linha de forma exponencial, como se mostra adiante no texto, de forma a modelar cenário mais realista no momento de aplicar a formulação a risers, onde se deve considerar atrito ao longo de seu comprimento apoiado. Evidentemente, esta hipótese "ad hoc" foi necessária, porque não se está discutindo o equilíbrio estático/dinâmico na direção longitudinal da viga.

Note-se, uma vez mais, que as equações segmentadamente lineares por domínio (83) e (84) com condições de contorno móveis, foram substituídas pela equação não linear (87) com condições de contorno fixas. 


\subsection{Obtenção do modelo de ordem reduzida}

A solução do problema é escrita na forma:

$u(z, \tau)=\hat{u}(z)+\delta(z, \tau)$,

sendo $\hat{u}(z)$ a solução correspondente à configuração estática satisfazendo a:

$\frac{1}{4} \hat{u}^{I V}-\gamma c_{0}{ }^{2} \hat{u}^{\prime \prime}-\hat{u}^{\prime} \gamma^{\prime} c_{0}{ }^{2}+c_{0}{ }^{4}(1+H \hat{u})=0$.

Conforme já referido, assume-se que a função que representa a tração fica definida como:

$\gamma(z, \tau)=\Gamma(\tau) e^{-\theta(1+z)}$

com

$\Gamma(\tau)=\Gamma_{0}+\Gamma_{1} \cos (\Omega \tau)$

sendo $\Gamma_{0}=\frac{T_{0}}{2 \sqrt{\mu E I}}, \Gamma_{1}=\frac{T_{1}}{2 \sqrt{\mu E I}}$ e $\Omega=\frac{\Omega_{d}}{\beta}$.

Tal hipótese para a tração considera um decaimento exponencial ao longo do comprimento. Alguns autores, como, por exemplo, Aranha e Pinto [1], adotam a hipótese de decaimento linear em linha com a suposição de atrito Coulomb ao longo do contato viga-apoio elástico, ao invés do decaimento exponencial aqui proposto. $\mathrm{O}$ anexo 7.3 discute a calibração dos dois modelos de decaimento.

Preferiu-se, portanto, adotar uma lei de decaimento da tração única, tanto para o trecho suspenso, onde ocorre diminuição da tração principalmente em função da geometria, como para o trecho apoiado, onde há atrito com o solo. 
A função que caracteriza a tração adimensional na configuração de equilíbrio é dada por:

$\gamma_{0}(z, \tau=0)=\Gamma_{0} e^{-\theta(1+z)}$.

Substituindo (93) em (90), decorre para a solução de equilíbrio:

$\frac{1}{4} \hat{u}^{I V}+c_{0}^{4}(1+H \hat{u})-\gamma_{0} c_{0}{ }^{2} \hat{u}^{\prime \prime}+\theta \gamma_{0} c_{0}{ }^{2} \hat{u}^{\prime}=0$,

ou seja:

$\frac{1}{4} \hat{u}^{I V}=\gamma_{0} c_{0}{ }^{2} \hat{u}^{\prime \prime}-\theta \gamma_{0} c_{0}{ }^{2} \hat{u}^{\prime}-c_{0}{ }^{4}(1+H \hat{u})$.

No que se segue, adota-se, entretanto, uma simplificação, qual seja $\hat{u}$ como em (13) e (14), ao considerar que a solução para a configuração estática no modelo de viga com flexão simples possa ser usada no modelo de viga sob flexão composta. Esta hipótese é tanto melhor, quanto menores forem as trações $T$ (ou $\gamma)$.

Incorporando a solução do equilíbrio (95) e levando em consideração (89) e (87) no desenvolvimento da equação, vem:

$$
\begin{aligned}
\frac{1}{4} \delta^{I V}-2 \dot{\delta}^{\prime} \dot{c}(\mathrm{z} & +1) c^{3}+\delta^{\prime \prime} c^{2}\left[\dot{c}^{2}(1+z)^{2}-\gamma\right]+\hat{u}^{\prime \prime}\left[c^{2} \dot{c}^{2}(z+1)^{2}-c^{2} \gamma+\gamma_{0} c_{0}{ }^{2}\right] \\
& +\delta^{\prime}\left[(z+1)\left(2 \dot{c}^{2}-\ddot{c} c\right)+\theta \gamma\right] c^{2} \\
& +\hat{u}^{\prime}\left[(z+1)\left(2 c^{2} \dot{c}^{2}-\ddot{c} c^{3}\right)+\theta \gamma c^{2}-\theta \gamma_{0} c_{0}{ }^{2}\right]+c^{4} \ddot{\delta}+H c^{4} \delta \\
& +c^{4}(1+H \hat{u})-c_{0}{ }^{4}(1+H \hat{u})=0 .
\end{aligned}
$$

A determinação dos modos de vibração para o problema da flexão composta e contato unilateral elástico, por procedimento análogo ao descrito no capítulo 2, demonstra-se de difícil abordagem. Assim, em nova aproximação, partindo-se da premissa que a solução utilizada para o modelo em que se 
considera a tração possa ser construída a partir da solução com flexão simples, projeta-se (96) segundo os modos de vibração apresentados na seção 2.2., de forma a obter a expressão do modelo de ordem reduzida não linear, de um grau de liberdade, cuja variável modal é o deslocamento horizontal do TDP, $U$. Faz-se uso, portanto, do método de Galerkin, em que a solução em um espaço linear normalizado é aproximada pela combinação linear de elementos deste mesmo espaço. Neste contexto, cita-se o trabalho desenvolvido em Scheidl et al [33]. No caso, com um único elemento (modo de vibração), escreve-se simplesmente:

$\delta=\zeta(z) U$

$c=c_{0}+U$,

onde $\zeta(z)$ é o modo de vibração obtido para o problema da viga com flexão simples com contato unilateral em apoio elástico, definido em (75) e (76).

Introduzindo (97) e (98) em (96), obtém-se:

$$
\begin{aligned}
& \frac{1}{4} \zeta^{I V} U-2 \zeta^{\prime} \dot{U}^{2}(\mathrm{z}+1)\left(c_{0}+U\right)^{3}+\zeta^{\prime \prime} U\left(c_{0}+U\right)^{2}\left[\dot{U}^{2}(1+z)^{2}\right. \\
&\quad-\gamma]+\hat{u}^{\prime \prime}\left[\left(c_{0}+U\right)^{2} \dot{U}^{2}(1+z)^{2}-\left(c_{0}+U\right)^{2} \gamma+\gamma_{0} c_{0}{ }^{2}\right] \\
& \quad+\zeta^{\prime} U\left\{(z+1)\left[2 \dot{U}^{2}-\ddot{U}\left(c_{0}+U\right)\right]+\theta \gamma\right\}\left(c_{0}+U\right)^{2} \\
& \quad+\hat{u}^{\prime}\left\{(z+1)\left[2\left(c_{0}+U\right)^{2} \dot{U}^{2}-\ddot{U}\left(c_{0}+U\right)^{2}\right]+\theta \gamma\left(c_{0}+U\right)^{2}-\theta \gamma_{0} c_{0}{ }^{2}\right\} \\
&+\left(c_{0}+U\right)^{4} \zeta \ddot{U}+H\left(c_{0}+U\right)^{4} \zeta U+\left(c_{0}+U\right)^{4}(1+H \hat{u})-c_{0}{ }^{4}(1+H \hat{u}) \\
&=0 .
\end{aligned}
$$

Com o intuito de completar a projeção iniciada em (99), considera-se cada termo desta equação como uma força $F(U, \dot{U}, \ddot{U}, \tau)$. Calculam-se, agora, os trabalhos virtuais de cada uma destas forças, no modelo de ordem reduzida de um grau de liberdade. Para tanto, realiza-se o produto $\delta W=F(\zeta \delta U)$, de sorte que tudo se passa como se todos os termos de (99) fossem multiplicados por $\zeta$. Sendo o deslocamento virtual $\delta U$ arbitrário, pode-se escrever: 


$$
\begin{aligned}
& \ddot{U}\left[\zeta c_{0}^{4}-(1+z) c_{0}^{3} \hat{u}^{\prime}\right] \zeta \\
& +U\left[\frac{1}{4} \zeta^{I V}+4 H \hat{u} c_{0}^{3}+H \zeta c_{0}^{4}+4 c_{0}^{3}-\gamma c_{0}^{2} \zeta^{\prime \prime}+\gamma \theta c_{0}^{2} \zeta^{\prime}-2 \gamma c_{0} \hat{u}^{\prime \prime}+2 \gamma \theta c_{0} \hat{u}^{\prime}\right] \zeta \\
& -\zeta\left(\gamma-\gamma_{0}\right) c_{0}^{2} \hat{u}^{\prime \prime}+\zeta \theta c_{0}^{2}\left(\gamma-\gamma_{0}\right) \hat{u}^{\prime} \\
& +U^{2}\left[6 c_{0}^{2}+6 H \hat{u} c_{0}^{2}+4 H \zeta c_{0}^{3}-2 \gamma c_{0} \zeta^{\prime \prime}+2 \gamma \theta c_{0} \zeta^{\prime}-\gamma \hat{u}^{\prime \prime}+\gamma \theta \hat{u}^{\prime}\right] \zeta \\
& +\dot{U}^{2}\left[(1+z)^{2} c_{0}^{2} \hat{u}^{\prime \prime}-2(1+z) c_{0}^{3} \zeta^{\prime}+2(1+z) c_{0}^{2} \hat{u}^{\prime}\right] \zeta \\
& +U \ddot{U}\left[4 \zeta c_{0}^{3}-(1+z) c_{0}^{3} \zeta^{\prime}-3(1+z) c_{0}^{2} \hat{u}^{\prime}\right] \zeta \\
& +U^{3}\left[4 c_{0}+4 H \hat{u} c_{0}+6 H \zeta c_{0}^{2}-\gamma \zeta^{\prime \prime}+\gamma \theta \zeta^{\prime}\right] \zeta \\
& +U \dot{U}^{2}\left[(1+z)^{2} c_{0}^{2} \zeta^{\prime \prime}-4(1+z) c_{0}^{2} \zeta^{\prime}+2(1+z)^{2} c_{0} \hat{u}^{\prime \prime}+4(1+z) c_{0} \hat{u}^{\prime}\right] \zeta \\
& \left.+U^{2} \ddot{U}\left[6 \zeta c_{0}^{2}-3(1+z) c_{0}^{2} \zeta^{\prime}-3(1+z) c_{0} \hat{u}^{\prime}\right)\right] \zeta \\
& +U^{4}\left[1+H \hat{u}+4 H \zeta c_{0}\right] \zeta \\
& +U^{2} \dot{U}^{2}\left[2(1+z)^{2} c_{0} \zeta^{\prime \prime}-2(1+z) c_{0} \zeta^{\prime}+(1+z)^{2} \hat{u}^{\prime \prime}+2(1+z) \hat{u}^{\prime}\right] \zeta \\
& +U^{3} \ddot{U}\left[4 \zeta c_{0}-3(1+z) c_{0} \zeta^{\prime}-(1+z) \hat{u}^{\prime}\right] \zeta \\
& +U^{5}(H \zeta) \zeta \\
& +U^{3} \dot{U}^{2}\left[(1+z)^{2} \zeta^{\prime \prime}\right] \zeta \\
& +U^{4} \ddot{U}\left[\zeta-(1+z) \zeta^{\prime}\right] \zeta=0 .
\end{aligned}
$$

Note-se que a equação (100), que pode ser entendida como a equação forçada do oscilador modal, apresenta coeficientes variáveis com $z$ e $\tau$. Com o intuito de eliminar a dependência destes coeficientes com $z$, definem-se constantes auxiliares $I_{i}, i=1$ a 13, definidos como os valores médios de integrais calculadas ao longo de um comprimento dimensional $L$ da viga, além do qual a função $\zeta(z)$ que caracteriza o modo, ou a função $\hat{u}(z)$, que caracteriza a configuração de equilíbrio ou ainda a função $\gamma(z, \tau)$, que caracteriza a tração, apresentam valores desprezíveis (a determinação do comprimento dimensional $L$ será abordada no capítulo 4). 


$$
\begin{aligned}
& I_{1}=\frac{1}{1+\frac{\alpha L}{c_{0}}} \int_{-1}^{\frac{\alpha L}{c_{0}}} \zeta \cdot \zeta d z \\
& I_{2}=\frac{1}{1+\frac{\alpha L}{c_{0}}} \int_{-1}^{\frac{\alpha L}{c_{0}}} \zeta \cdot \zeta^{I V} d z \\
& I_{3}=\frac{1}{1+\frac{\alpha L}{c_{0}}} \int_{-1}^{\frac{\alpha L}{c_{0}}} \zeta \cdot \zeta^{\prime}(1+z) d z \\
& I_{4}=\frac{1}{1+\frac{\alpha L}{c_{0}}} \int_{-1}^{\frac{\alpha L}{c_{0}}} \zeta d z \\
& I_{5}=\frac{1}{1+\frac{\alpha L}{c_{0}}} \int_{-1}^{\frac{\alpha L}{c_{0}}} \zeta \cdot \zeta^{\prime \prime}(1+z)^{2} d z \\
& I_{6}=\frac{1}{1+\frac{\alpha L}{c_{0}}} \int_{-1}^{\frac{\alpha L}{c_{0}}} \zeta \cdot \hat{u}^{\prime}(1+z) d z \\
& I_{7}=\frac{1}{1+\frac{\alpha L}{c_{0}}} \int_{-1}^{\frac{\alpha L}{c_{0}}} \zeta \cdot \hat{u}^{\prime \prime}(1+z)^{2} d z \\
& I_{8}=\frac{1}{1+\frac{\alpha L}{c_{0}}} \int_{-1}^{\frac{\alpha L}{c_{0}}} \zeta \cdot \zeta^{\prime \prime} e^{-\theta(1+z)} d z \\
& I_{9}=\frac{1}{1+\frac{\alpha L}{c_{0}}} \int_{-1}^{\frac{\alpha L}{c_{0}}} \zeta \cdot \hat{u}^{\prime \prime} e^{-\theta(1+z)} d z \\
& I_{10}=\frac{1}{1+\frac{\alpha L}{c_{0}}} \int_{-1}^{\frac{\alpha L}{c_{0}}} \zeta \cdot \zeta^{\prime} e^{-\theta(1+z)} d z \\
& I_{11}=\frac{1}{1+\frac{\alpha L}{c_{0}}} \int_{-1}^{\frac{\alpha L}{c_{0}}} \zeta \cdot \hat{u}^{\prime} e^{-\theta(1+z)} d z \\
& I_{12}=\frac{1}{1+\frac{\alpha L}{c_{0}}} \int_{-1}^{\frac{\alpha L}{c_{0}}} \zeta \cdot H \zeta d z \\
& I_{13}=\frac{1}{1+\frac{\alpha L}{c_{0}}} \int_{-1}^{\frac{\alpha L}{c_{0}}} \zeta \cdot H \hat{u} d z
\end{aligned}
$$


Assim, a equação (99) pode ser escrita na forma:

$$
\begin{aligned}
& \ddot{U}\left[I_{1} c_{0}^{4}-c_{0}^{3} I_{6}\right] \\
& +U\left[\frac{1}{4} I_{2}+4 c_{0}^{3} I_{13}+c_{0}^{4} I_{12}-\Gamma c_{0}^{2} I_{8}+\theta c_{0}^{2} \Gamma I_{10}-2 c_{0} \Gamma I_{9}+2 \theta c_{0} \Gamma I_{11}+4 c_{0}^{3} I_{4}\right] \\
& -\left(\Gamma-\Gamma_{0}\right) c_{0}^{2} I_{9}+\theta c_{0}^{2}\left(\Gamma-\Gamma_{0}\right) I_{11} \\
& +U^{2}\left[6 c_{0}^{2} I_{4}+6 c_{0}^{2} I_{13}+4 c_{0}^{3} I_{12}-2 c_{0} \Gamma I_{8}+2 \theta c_{0} \Gamma I_{10}-\Gamma I_{9}+\theta \Gamma I_{11}\right] \\
& +\dot{U}^{2}\left[c_{0}^{2} I_{7}-2 c_{0}^{3} I_{3}+2 c_{0}^{2} I_{6}\right] \\
& +U \ddot{U}\left[4 c_{0}^{3} I_{1}-c_{0}^{3} I_{3}-3 c_{0}^{2} I_{6}\right] \\
& +U^{3}\left[4 c_{0} I_{4}+4 c_{0} I_{13}+6 c_{0}^{2} I_{12}-\Gamma I_{8}+\theta \Gamma I_{10}\right] \\
& +U \dot{U}^{2}\left[c_{0}^{2} I_{5}-4 c_{0}^{2} I_{3}+2 c_{0} I_{7}+4 c_{0} I_{6}\right] \\
& +U^{2} \ddot{U}\left[6 c_{0}^{2} I_{1}-3 c_{0}^{2} I_{3}-3 c_{0} I_{6}\right] \\
& +U^{4}\left[I_{4}+I_{13}+4 c_{0} I_{12}\right] \\
& +U^{2} \dot{U}^{2}\left[2 c_{0} I_{5}-2 c_{0} I_{3}+I_{7}+2 I_{6}\right] \\
& +U^{3} \ddot{U}\left[4 c_{0} I_{1}-3 c_{0} I_{3}-I_{6}\right] \\
& +U^{5}\left[I_{12}\right] \\
& +U^{3} \dot{U}^{2}\left[I_{5}\right] \\
& +U^{4} \ddot{U}\left[I_{1}-I_{3}\right]=0 .
\end{aligned}
$$

Definem-se os coeficientes $a_{i, i}=0 a 2$, dos termos lineares, sendo:

$a_{0}=I_{1} c_{0}^{4}-c_{0}^{3} I_{6}$

a massa modal;

$a_{1}=2 \xi \omega a_{0}$

o coeficiente de amortecimento viscoso linear equivalente, introduzido de forma "ad hoc", uma vez que não decorre da formulação inicial do problema;

$$
\begin{gathered}
a_{2}=\left[\frac{1}{4} I_{2}+4 c_{0}^{3} I_{13}+4 c_{0}^{3}+c_{0}^{4} I_{12}-\Gamma\left(c_{0}^{2} I_{8}-\theta c_{0}^{2} I_{10}+2 c_{0} I_{9}-2 \theta c_{0} I_{11}\right)\right] \\
=a_{2,0}+a_{2,1} \cos (\Omega \tau)
\end{gathered}
$$


com

$$
\begin{aligned}
& a_{2,0}=\frac{1}{4} I_{2}+4 c_{0}^{3} I_{13}+4 c_{0}^{3}+c_{0}^{4} I_{12}-\Gamma_{0}\left[c_{0}^{2} I_{8}-\theta c_{0}^{2} I_{10}+2 c_{0} I_{9}-2 \theta c_{0} I_{11}\right] \\
& a_{2,1}=-\Gamma_{1}\left[c_{0}^{2} I_{8}-\theta c_{0}^{2} I_{10}+2 c_{0} I_{9}-2 \theta c_{0} I_{11}\right]
\end{aligned}
$$

a rigidez modal do problema com flexo-tração, representando $a_{2,0}$ a parcela referente à solução estática e $a_{2,1}$ a variação dinâmica.

Definem-se, ainda, os coeficientes $b_{i,} i=0 a 12$, em sua forma geral, como sendo característicos do carregamento forçado, separando-se a contribuição da configuração estática daquela oriunda da perturbação dinâmica em função do tempo:

$b_{i}=b_{i, 0}+b_{i, 1} \cos (\Omega \tau)$,

com

$$
\begin{aligned}
& b_{0,0}=0 \\
& b_{0,1}=\Gamma_{1}\left(c_{0}^{2} I_{9}-\theta c_{0}^{2} I_{11}\right) \\
& b_{1,0}=-6 c_{0}^{2} I_{4}-6 c_{0}^{2} I_{13}-4 c_{0}^{3} I_{12}+\Gamma_{0}\left[2 c_{0} I_{8}-2 \theta c_{0} I_{10}+I_{9}-\theta I_{11}\right] \\
& b_{1,1}=\Gamma_{1}\left[2 c_{0} I_{8}-2 \theta c_{0} I_{10}+I_{9}-\theta I_{11}\right] \\
& b_{2,0}=-c_{0}^{2} I_{7}+2 c_{0}^{3} I_{3}-2 c_{0}^{2} I_{6} \\
& b_{2,1}=0 \\
& b_{3,0}=-4 c_{0}^{3} I_{1}+c_{0}^{3} I_{3}+3 c_{0}^{2} I_{6} \\
& b_{3,1}=0 \\
& b_{4,0}=-4 c_{0} I_{4}-4 c_{0} I_{13}-6 c_{0}^{2} I_{12}+\Gamma_{0}\left[I_{8}-\theta I_{10}\right] \\
& b_{4,1}=\Gamma_{1}\left[I_{8}-\theta I_{10}\right] \\
& b_{5,0}=-c_{0}^{2} I_{5}+4 c_{0}^{2} I_{3}-2 c_{0} I_{7}-4 c_{0} I_{6} \\
& b_{5,1}=0 \\
& b_{6,0}=-6 c_{0}^{2} I_{1}+3 c_{0}^{2} I_{3}+3 c_{0} I_{6} \\
& b_{6,1}=0
\end{aligned}
$$




$$
\begin{aligned}
& b_{7,0}=-I_{4}-I_{13}-4 c_{0} I_{12} \\
& b_{7,1}=0 \\
& b_{8,0}=-2 c_{0} I_{5}+2 c_{0} I_{3}-I_{7}-2 I_{6} \\
& b_{8,1}=0 \\
& b_{9,0}=-4 c_{0} I_{1}+3 c_{0} I_{3}+I_{6} \\
& b_{9,1}=0 \\
& b_{10,0}=-I_{12} \\
& b_{10,1}=0 \\
& b_{11,0}=-I_{5} \\
& b_{11,1}=0 \\
& b_{12,0}=-I_{1}+I_{3} \\
& b_{12,1}=0 .
\end{aligned}
$$

Podemos reescrever a equação (102) na forma de um oscilador não-linear forçado:

$$
\begin{aligned}
a_{0} \ddot{U}+a_{1} \dot{U}+ & a_{2} U \\
& =b_{0}+b_{1} U^{2}+b_{2} \dot{U}^{2}+b_{3} U \ddot{U}+b_{4} U^{3}+b_{5} U \dot{U}^{2}+b_{6} U^{2} \ddot{U}+b_{7} U^{4} \\
& +b_{8} U^{2} \dot{U}^{2}+b_{9} U^{3} \ddot{U}+b_{10} U^{5}+b_{11} U^{3} \dot{U}^{2}+b_{12} U^{4} \ddot{U},
\end{aligned}
$$

ou ainda

$$
\begin{aligned}
\ddot{U}+\hat{a}_{1} \dot{U}+\left[\hat{a}_{2,0}\right. & \left.+\hat{a}_{2,1} \cos (\Omega \tau)\right] U \\
& =\hat{b}_{0,1} \cos (\Omega \tau)+\left[\hat{b}_{1,0}+\hat{b}_{1,1} \cos (\Omega \tau)\right] U^{2}+\hat{b}_{2,0} \dot{U}^{2}+\hat{b}_{3,0} U \ddot{U} \\
& +\left[\hat{b}_{4,0}+\hat{b}_{4,1} \cos (\Omega \tau)\right] U^{3}+\hat{b}_{5,0} U \dot{U}^{2}+\hat{b}_{6,0} U^{2} \ddot{U}+\hat{b}_{7,0} U^{4}+\hat{b}_{8,0} U^{2} \dot{U}^{2} \\
& +\hat{b}_{9,0} U^{3} \ddot{U}+\hat{b}_{10,0} U^{5}+\hat{b}_{11,0} U^{3} \dot{U}^{2} \\
& +\hat{b}_{12,0} U^{4} \ddot{U}
\end{aligned}
$$

sendo:

$\hat{a}_{1}=\frac{a_{1}}{a_{0}}, \quad \hat{a}_{2,0}=\frac{a_{2,0}}{a_{0}}, \quad \hat{a}_{2,1}=\frac{a_{2,1}}{a_{0}}$, 
$\hat{b}_{i, 0}=\frac{b_{i, 0}}{a_{0}}, i=1$ a 12 ,

e

$\hat{b}_{i, 1}=\frac{b_{i, 1}}{a_{0}}, i=1$ a 12 .

Observa-se que a equação (111) apresenta termos na forma $\left[\hat{b}_{i, 0}+\right.$ $\left.\hat{b}_{i, 1} \cos (\Omega \tau)\right] U^{n}, n=2 a 4$, que a fazem diferir da equação clássica de Mathieu não forçada $\ddot{U}+\omega^{2}[1+\eta \cos (\Omega \tau)] U=0, \eta \ll 1$. Verifica-se, portanto, que a equação (111) possui termos paramétricos variando em vários coeficientes além de forçamento direto. 


\section{Estudo de caso}

\subsection{Análise paramétrica}

De posse do modelo de ordem reduzida descrito em (111), pretende-se simular através de integração numérica, utilizando para tanto o método RungeKutta de $4^{\mathrm{a}}$ ordem, o comportamento do oscilador em casos onde ocorra excitação paramétrica.

Primeiramente, pretende-se avaliar de forma essencialmente qualitativa o comportamento do modelo de ordem reduzida perante variações nos valores dos parâmetros do sistema e nas condições iniciais. Assim, os primeiros gráficos têm como condições para deslocamento inicial e velocidade inicial valores em termos da variável modal generalisada $U_{0}$ e sua derivada $\dot{U}_{0}=V_{0}$, variando-se a tensão estática $T_{0}$ no ponto "O" e a rigidez do solo por unidade de comprimento $\mu=\varphi D$, onde $\varphi$ representa a rigidez do solo por unidade de área e $D$ representa $o$ diâmetro da viga. Reserva-se para a seção 4.2. discussão com base em parâmetros dimensionais para as condições iniciais.

Para que ocorra o efeito de ressonância paramétrica clássica, do tipo regida pela equação de Mathieu, a freqüência excitante $\Omega$ deve ser um múltiplo da freqüência natural $\omega_{0}=\sqrt{\hat{a}_{2,0}}$ do modelo de ordem reduzida. $O$ presente estudo limita-se apenas a casos em que a freqüência excitante $\Omega$ é o dobro da freqüência natural $\omega_{0}$.

Os processamentos foram feitos tendo como base algumas propriedades de viga. A tabela Tab. 2 abaixo apresenta parâmetros do sistema que serão mantidos fixos: 


\begin{tabular}{|l|c|}
\hline Módulo de Young & $E=2.1 \times 10^{11} \mathrm{~N} / \mathrm{m}^{2}$ \\
\hline Diâmetro nominal & $D=0.2032 \mathrm{~m}$ \\
\hline Área da Seção Transversal & $A=1.1021 \times 10^{-2} \mathrm{~m}^{2}$ \\
\hline Momento de Inércia & $I=4.72143 \times 10^{-5} \mathrm{~m}^{4}$ \\
\hline Massa por unidade de comprimento (incluindo a massa adicional) & $\rho=141.24 \mathrm{~kg} / \mathrm{m}$ \\
\hline Peso próprio submerso da viga por unidade de comprimento & $p=727 \mathrm{~N} / \mathrm{m}$ \\
\hline
\end{tabular}

Tabela 2: Propriedades físicas da viga considerada no estudo de caso de análise paramétrica, ver Pesce et al [28]

O parâmetro $\theta$ presente nas equações (91) e (93), que ilustram o efeito da tração na formulação do problema, foi determinado admitindo-se que o decaimento exponencial a partir da força normal no ponto "O", $N_{0}$, ao longo do comprimento em que ocorre atrito com o solo $L$, passa a ser $1 \% N_{0}$. Para maiores detalhes, vide anexo 7.3.

O coeficiente de rigidez do solo $\varphi$ tem papel fundamental no desenvolvimento do estudo de caso da excitação paramétrica. Os valores considerados neste estudo de caso foram $\varphi=10^{2} \mathrm{~N} / \mathrm{m}^{3}, \varphi=10^{3} \mathrm{~N} / \mathrm{m}^{3}$ e $\varphi=$ $10^{4} \mathrm{~N} / \mathrm{m}^{3}$.

Para o estudo de caso, foram utilizados valores de tração estática $T_{0, T D P}$ no TDP que variaram de $10 \mathrm{kN}$ a $45 \mathrm{kN}$.

Para obtenção dos modos de vibração utilizados neste estudo de caso, e nos estudos de caso na sessão 4.2., foi utilizada a seguinte rotina de cálculo, tendo como base Pesce et al [28], a partir de parâmetros da Tab. 2:

- Obtenção da escala de comprimento flexional, para o qual a rigidez flexional do riser apresenta relevância:

$$
\lambda=\sqrt{\frac{E I}{T_{0, T D P}}} ;
$$


- determinação da abscissa estática dimensional do TDP em relação à extremidade da viga semi-infinita (valor aproximado), representado na Fig. 14:

$x_{c}=4 \lambda ; \mathrm{e}$

- determinação da posição do TDP na sua forma adimensional, a partir do valor de $\alpha$, definido em (81):

$c_{0}=\alpha x_{c}$.

Portanto, a partir da tração estática $T_{0, T D P}$ no $T D P$, da rigidez flexional do riser $E I$, e do coeficiente de rigidez do solo $\varphi$, é possível determinar a posição do TDP em relação à extremidade da viga semi-infinita e, assim, calcular os modos de vibração associados a esta posição, tal como se apresenta na Tab. 3 abaixo, os modos de vibração obtidos para o modelo de viga sob flexão simples:

\begin{tabular}{|c|c|c|c|c|}
\hline$\varphi\left(N / m^{3}\right)$ & $T_{0, T D P}(k N)$ & $c_{0}$ & $\eta=\sqrt[4]{\frac{1-\omega^{2}}{\omega^{2}}}$ & $\omega$ \\
\hline \multirow{2}{*}{$10^{4}$} & \multirow{2}{*}{45} & \multirow{2}{*}{4.96244} & 2.14934 & 0.211567 \\
\hline & & & 1.09289 & 0.64195 * \\
\hline \multirow{3}{*}{$10^{4}$} & \multirow{3}{*}{40} & \multirow{3}{*}{5.26347} & 2.25856 & 0.192375 \\
\hline & & & 1.16997 & $0.589902^{*}$ \\
\hline & & & 0.254452 & 0.997911 \\
\hline \multirow{3}{*}{$10^{4}$} & \multirow{3}{*}{30} & \multirow{3}{*}{6.07773} & 2.55303 & 0.151648 \\
\hline & & & 1.36323 & $0.473853^{*}$ \\
\hline & & & 0.695264 & 0.900328 \\
\hline \multirow{2}{*}{$10^{3}$} & \multirow{2}{*}{30} & \multirow{2}{*}{3.41776} & 1.58076 & 0.371545 \\
\hline & & & 0.534035 & 0.961656 * \\
\hline \multirow{2}{*}{$10^{3}$} & \multirow{2}{*}{25} & \multirow{2}{*}{3.74397} & 1.7027 & 0.326071 \\
\hline & & & 0.698503 & 0.898733 * \\
\hline \multirow{2}{*}{$10^{2}$} & \multirow{2}{*}{10} & \multirow{2}{*}{3.32891} & 1.54724 & 0.385444 \\
\hline & & & 0.478846 & $0.974705^{*}$ \\
\hline
\end{tabular}

Tabela 3: Modos de vibração em função da rigidez do solo e tração estática no TDP 
Os modos marcados com $\left(^{*}\right)$ mostram quais os que foram utilizados nos processamentos, nos casos em que para um determinado valor de $c_{0}$ há mais de um modo de vibração.

A seguir, são apresentadas as imagens dos processamentos para cada caso, ordenados, pela ordem, segundo os parâmetros: coeficiente de rigidez do solo $\varphi$, a tração estática $T_{0, T D P}$ no TDP, a taxa de amortecimento $\xi$, o valor de $c_{0}$, o período $T$ em segundos associado à freqüência excitante.

Os valores em azul escuro no mapa de fase mostram a trajetória de fase ao final do período estudado, tentando assim evidenciar os casos em que existe um estado estacionário. 


\section{Equação do modelo de ordem reduzida para o caso T1:}

$\ddot{U}+[0.222379-0.004141 \cos (0.943143 \tau)] U$

$$
\begin{aligned}
& =0.026425 \cos (0.943143 \tau)+[0.088042 .+0.004665 \cos (0.943143 \tau)] U^{2} \\
& +0.056559 \dot{U}^{2}-1.119569 U \ddot{U}+[0.024389+0.001058 \cos (0.943143 \tau)] U^{3} \\
& +0.177035 U \dot{U}^{2}-0.467515 U^{2} \ddot{U}-0.002637 U^{4}+0.091051 U^{2} \dot{U}^{2}-0.086225 U^{3} \ddot{U} \\
& -0.000160 U^{5}+0.012909 U^{3} \dot{U}^{2}-0.005919 U^{4} \ddot{U}
\end{aligned}
$$
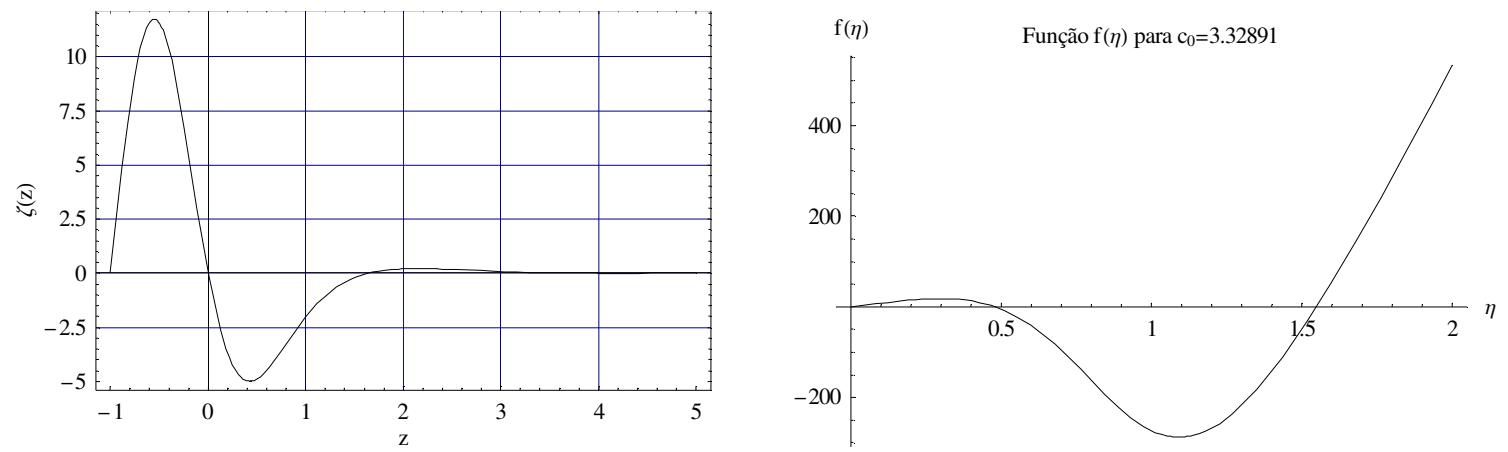

Figura 15: Funções modais $\zeta(z)$ e $f(\eta)$ para $\phi=10^{2} N / m^{3}, T_{0, T D P}=10 k N$ e $c_{0}=3.32891$
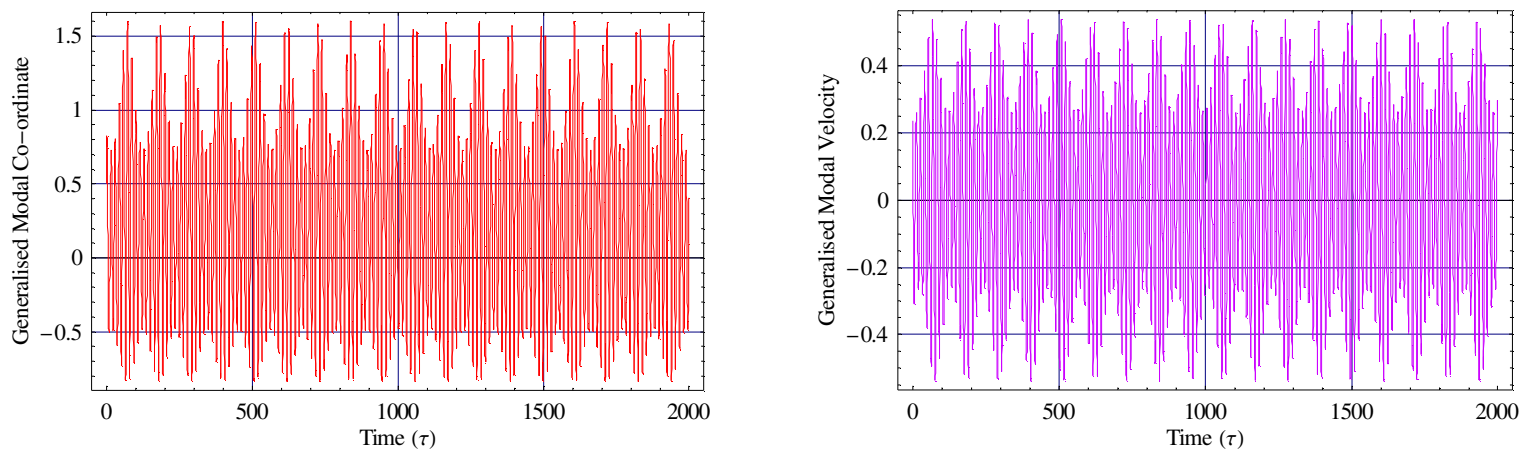

Figura 16: Deslocamento U e velocidade V para $\phi=10^{2} \mathrm{~N} / \mathrm{m}^{3}, \mathrm{~T}_{0, T D P=10 \mathrm{kN}}, \mathrm{T}_{1, T D P=15 \%} \mathrm{~T}_{0, T D P}, \xi=0 \%$, $\mathrm{C}_{0}=3.32891, \mathrm{~T}=17.6 \mathrm{~s}, \mathrm{U}_{0}=0.5$ e $\mathrm{V}_{0}=0.24$
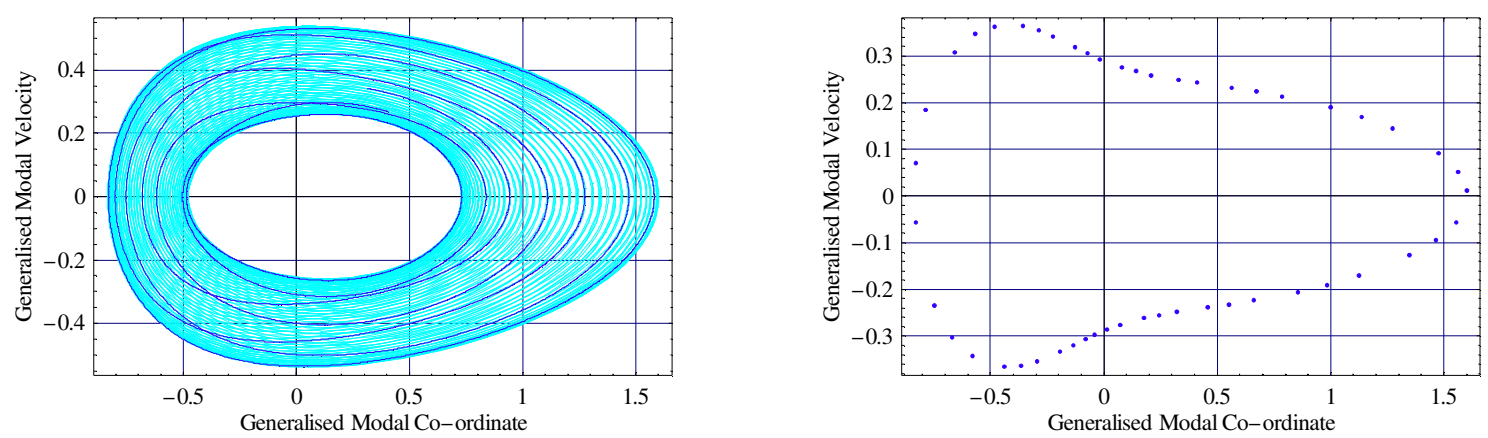

Figura 17: Mapa de fase e mapa de Poincaré para $\phi=10^{2} \mathrm{~N} / \mathrm{m}^{3}, \mathrm{~T}_{0, T D P}=10 \mathrm{kN}, \mathrm{T}_{1, T D P=15 \%} \mathrm{~T}_{0, T D P}, \xi=0 \%$, $\mathrm{C}_{0}=3.32891, \mathrm{~T}=17.6 \mathrm{~s}, \mathrm{U}_{0}=0.5$ e $\mathrm{V}_{0}=0.24$ 
Equação do modelo de ordem reduzida para o caso T2:

$$
\begin{aligned}
\ddot{U}+[0.222379- & 0.00138 \cos (0.943143 \tau)] U \\
& =0.008808 \cos (0.943143 \tau)+[0.088042+0.001555 \cos (0.943143 \tau)] U^{2} \\
& +0.056559 \dot{U}^{2}-1.119569 U \ddot{U}+[0.024389+0.000352 \cos (0.943143 \tau)] U^{3} \\
& +0.177035 U \dot{U}^{2}-0.467515 U^{2} \ddot{U}-0.002637 U^{4}+0.091051 U^{2} \dot{U}^{2}-0.086225 U^{3} \ddot{U} \\
& -0.00016 U^{5}+0.012909 U^{3} \dot{U}^{2}-0.005919 U^{4} \ddot{U}
\end{aligned}
$$
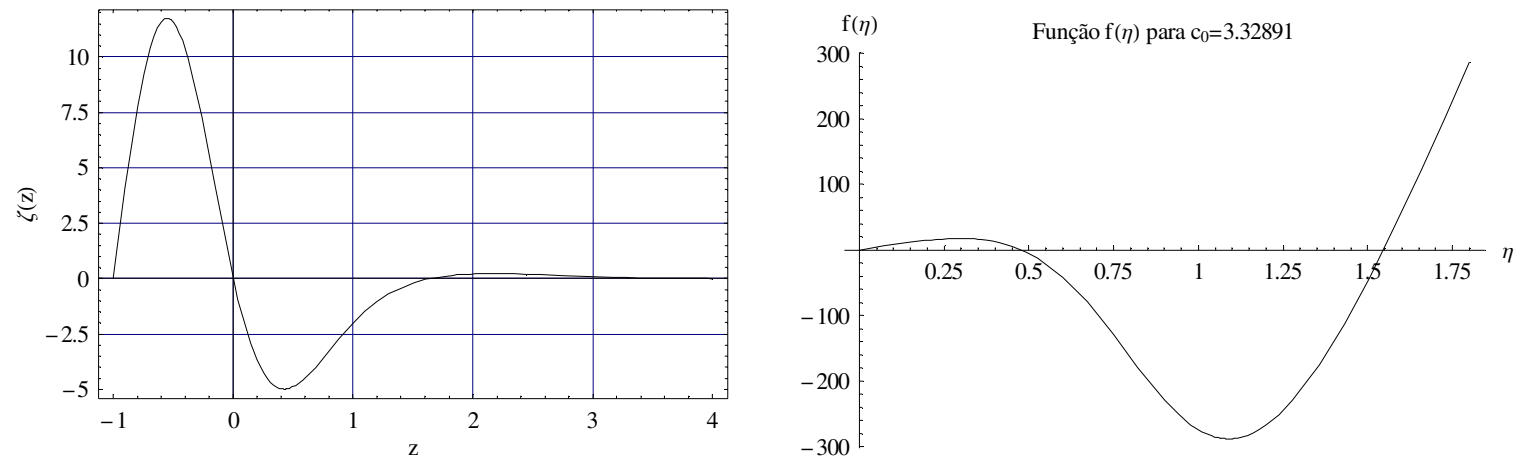

Figura 18: Funções modais $\zeta(z)$ e $f(\eta)$ para $\phi=10^{2} \mathrm{~N} / \mathrm{m}^{3}, \mathrm{~T}_{0, T D P}=10 \mathrm{kN}$ e $c_{0}=3.32891$
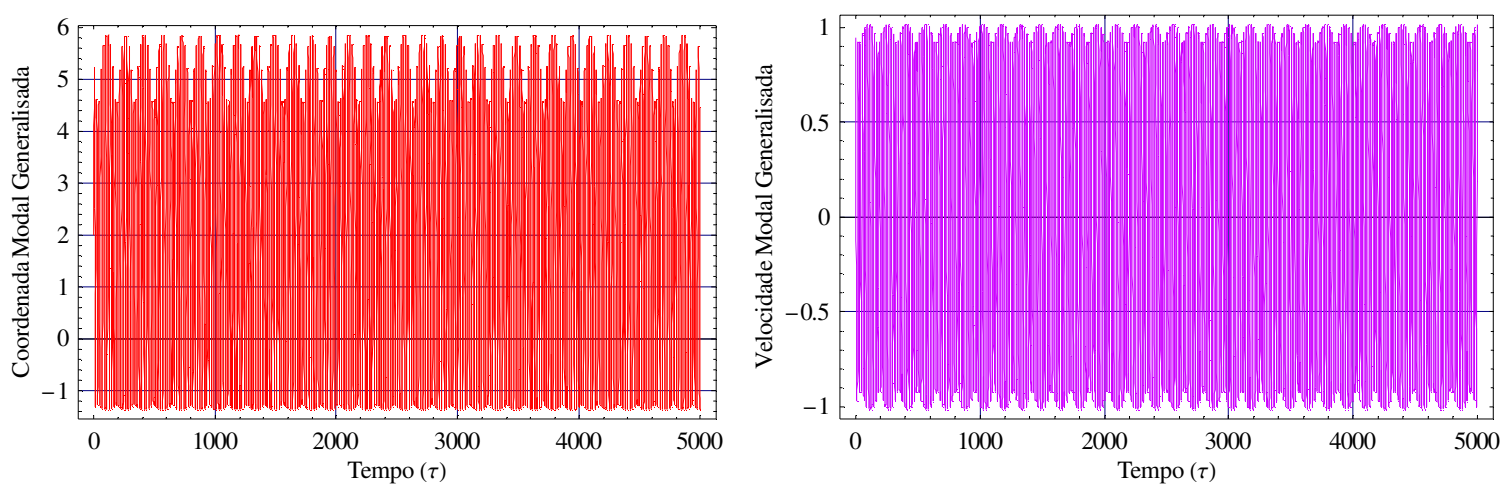

Figura 19: Deslocamento U e velocidade V para $\phi=10^{2} \mathrm{~N} / \mathrm{m}^{3}, \mathrm{~T}_{0, T D P}=10 \mathrm{kN}, \mathrm{T}_{1, T D P=5 \%} \mathrm{~T}_{0, T D P}, \xi=0 \%$, $\mathrm{C}_{0}=3.32891, \mathrm{~T}=17.6 \mathrm{~s}, \mathrm{U}_{0}=2$ e $\mathrm{V}_{0}=0.94$
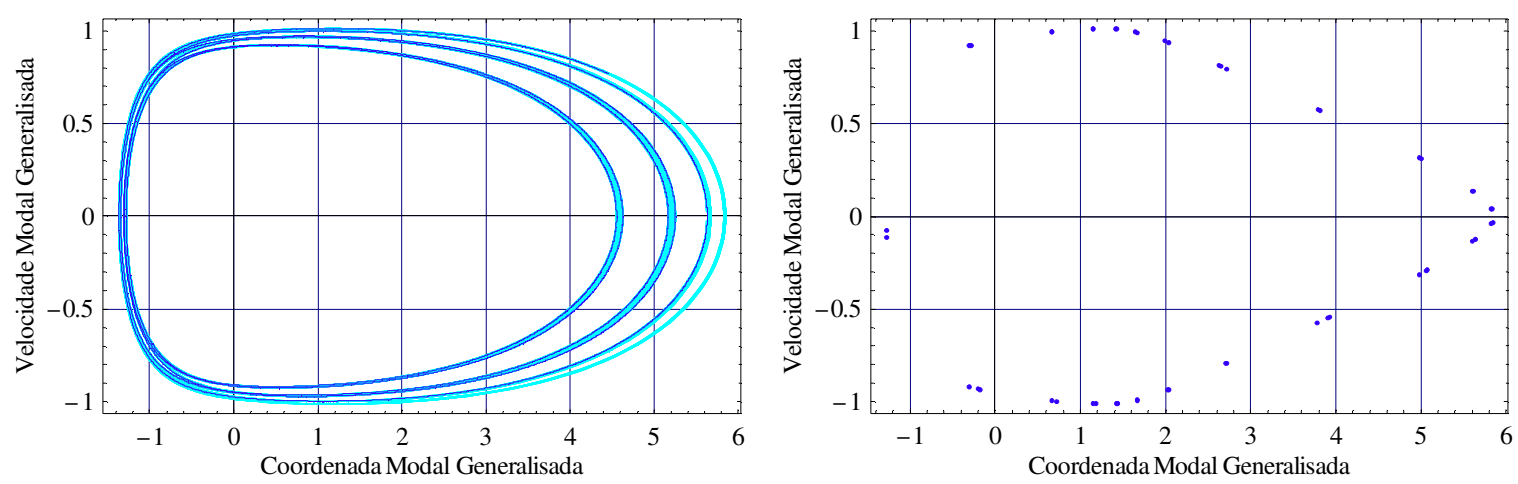

Figura 20: Mapa de fase e mapa de Poincaré para $\phi=10^{2} \mathrm{~N} / \mathrm{m}^{3}, \mathrm{~T}_{0, T D P=10 \mathrm{kN}}, \mathrm{T}_{1, T D P=5 \%} \mathrm{~T}_{0, T D P}, \xi=0 \%$, $\mathrm{C}_{0}=3.32891, \mathrm{~T}=17.6 \mathrm{~s}, \mathrm{U}_{0}=2$ e $\mathrm{V}_{0}=0.94$ 
Equação do modelo de ordem reduzida para o caso T3:

$$
\begin{aligned}
\ddot{U}+0.007413 \dot{U}+ & {[0.137389-0.007154 \cos (0.741322 \tau)] U } \\
& =0.050672 \cos (0.741322 \tau)+[0.096917+0.007023 \cos (0.741322 \tau)] U^{2} \\
& -0.007420 \dot{U}^{2}-1.019718 U \ddot{U}+[0.024551+0.001420 \cos (0.741322 \tau)] U^{3} \\
& +0.100264 U \dot{U}^{2}-0.389046 U^{2} \ddot{U}-0.002240 U^{4}+0.055148 U^{2} \dot{U}^{2}-0.065803 U^{3} \ddot{U} \\
& -0.000131 U^{5}-0.007435 U^{3} \dot{U}^{2}-0.004162 U^{4} \ddot{U}
\end{aligned}
$$
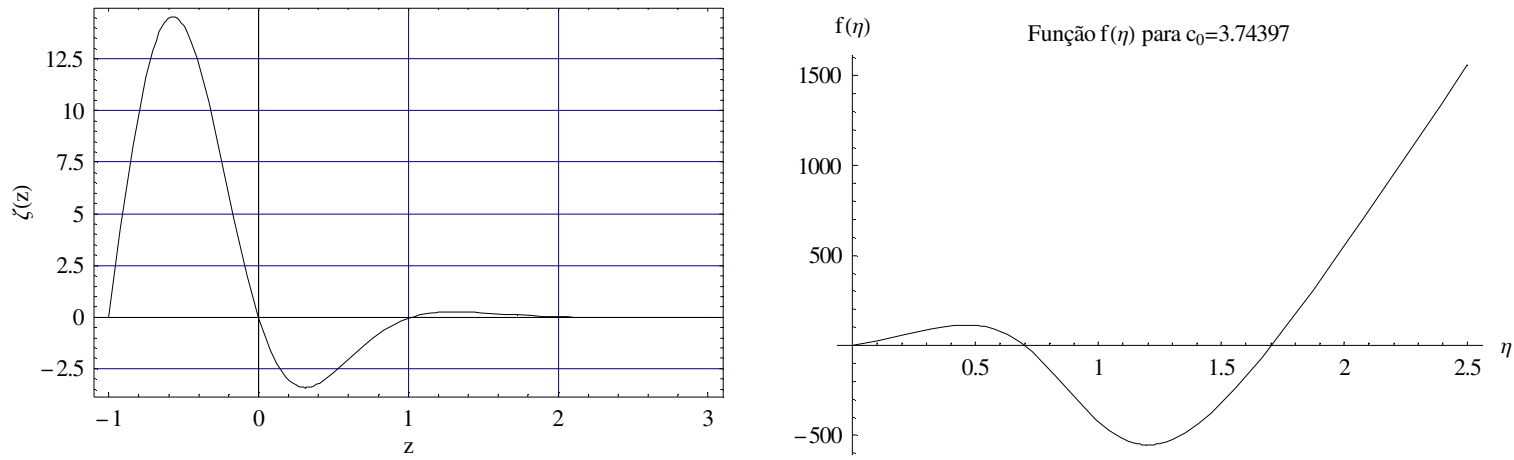

Figura 21: Funções modais $\zeta(z)$ e $f(\eta)$ para $\phi=10^{3} \mathrm{~N} / \mathrm{m}^{3}, \mathrm{~T}_{0, T D P}=25 \mathrm{kN}$ e $c_{0}=3.74397$
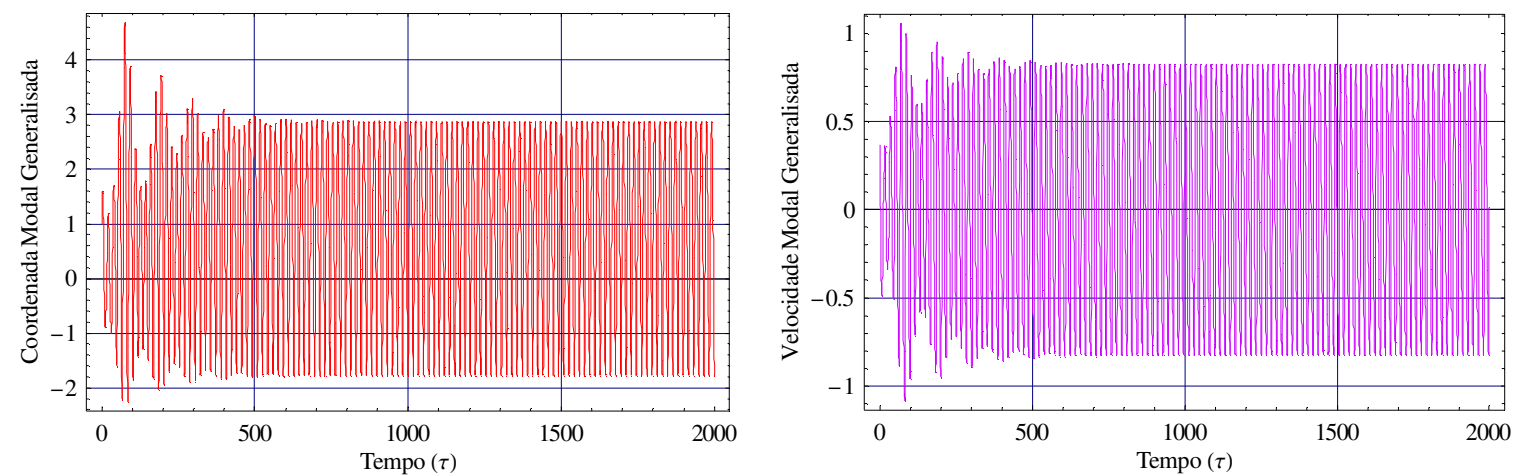

Figura 22: Deslocamento U e velocidade $V$ para $\phi=10^{3} \mathrm{~N} / \mathrm{m}^{3}, \mathrm{~T}_{0, T D P=25 \mathrm{kN}}, \mathrm{T}_{1, T D P=15 \%} \mathrm{~T}_{0, T D P}, \xi_{=1 \%}$, $C_{0}=3.74397, T=7.1 \mathrm{~s}, \mathrm{U}_{0}=1$ e $\mathrm{V}_{0}=0.37$
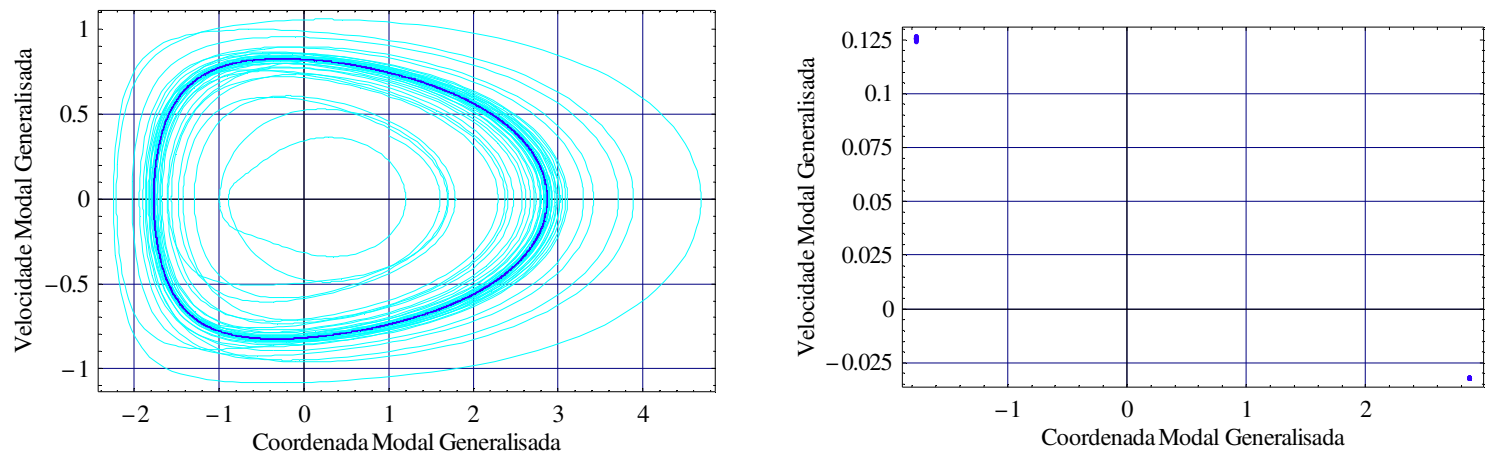

Figura 23: Mapa de fase e mapa de Poincaré para $\phi=10^{3} \mathrm{~N} / \mathrm{m}^{3}, \mathrm{~T}_{0, T D P}=25 \mathrm{kN}, \mathrm{T}_{1, T D P=15 \%} \mathrm{~T}_{0, T D P}, \xi=1 \%$, $\mathrm{C}_{0}=3.74397, \mathrm{~T}=7.1 \mathrm{~s}, \mathrm{U}_{0}=1$ e $\mathrm{V}_{0}=0.37$ 


\section{Equação do modelo de ordem reduzida para o caso T4:}

$$
\begin{aligned}
\ddot{U}+0.004756 \dot{U} & +[0.226211-0.00267 \cos (0.951234 \tau)] U \\
& =0.02138 \cos (0.951234 \tau)+[0.19913+0.003928 \cos (0.951234 \tau)] U^{2}-0.012999 \dot{U}^{2} \\
& -1.128674 U \ddot{U}+[0.061899+0.000842 \cos (0.951234 \tau)] U^{3}+0.1649 U \dot{U}^{2} \\
& -0.477063 U^{2} \ddot{U}-0.00802 U^{4}+0.099835 U^{2} \dot{U}^{2}-0.089487 U^{3} \ddot{U}-0.000553 U^{5} \\
& +0.014768 U^{3} \dot{U}^{2}-0.006284 U^{4} \ddot{U}
\end{aligned}
$$
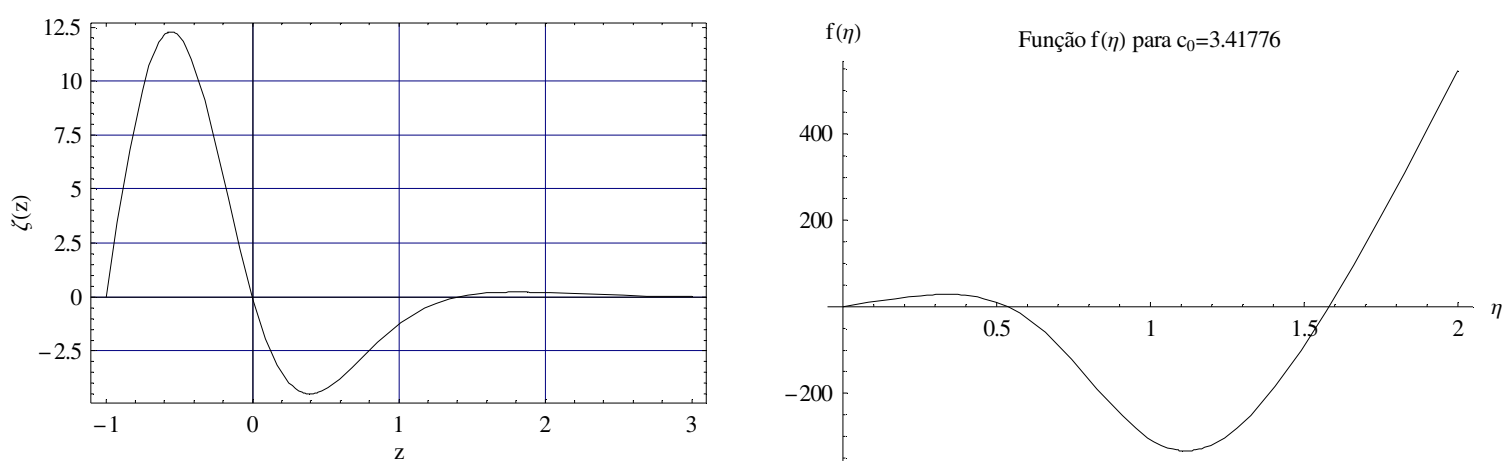

Figura 24: Funções modais $\zeta(z)$ e $f(\eta)$ para $\phi=10^{3} \mathrm{~N} / \mathrm{m}^{3}, \mathrm{~T}_{0, T D P}=30 \mathrm{kN}$ e $c_{0}=3.41776$
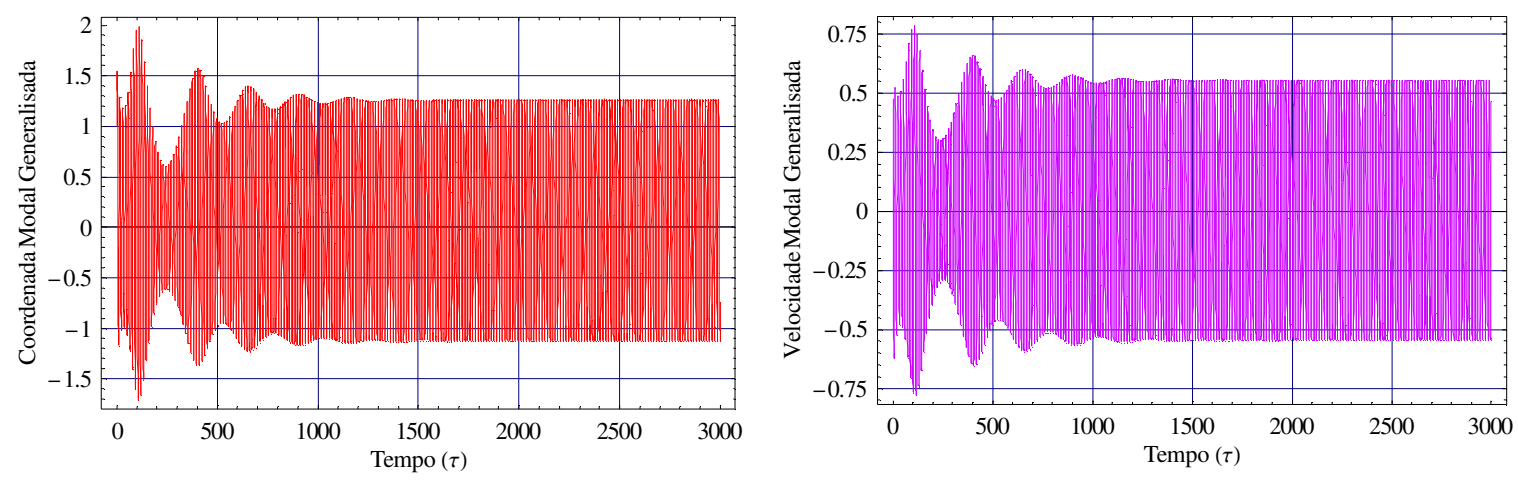

Figura 25: Deslocamento U e velocidade V para $\phi=10^{3} \mathrm{~N} / \mathrm{m}^{3}, \mathrm{~T}_{0, T D P=30 \mathrm{kN}}, \mathrm{T}_{1, T D P=5 \%} \mathrm{~T}_{0, T D P}, \xi=0.5 \%$, $\mathrm{C}_{0}=3.41776, \mathrm{~T}=5.5 \mathrm{~s}, \mathrm{U}_{0}=1$ e $\mathrm{V}_{0}=0.48$
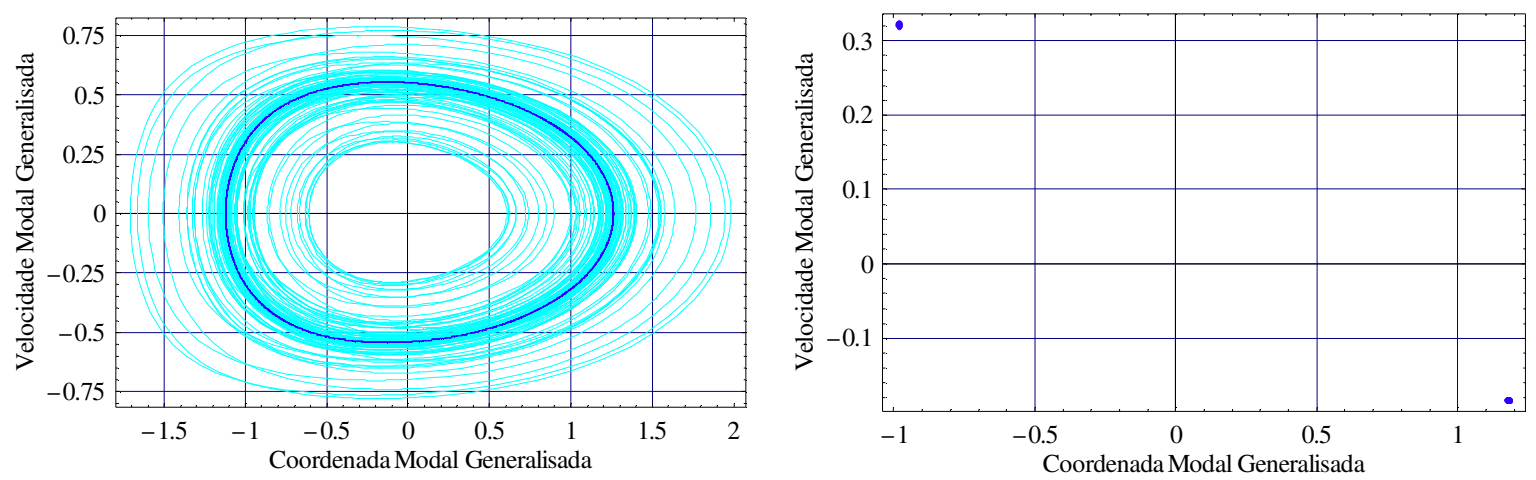

Figura 26: Mapa de fase e mapa de Poincaré para $\phi=10^{3} \mathrm{~N} / \mathrm{m}^{3}, \mathrm{~T}_{0, T D P}=30 \mathrm{kN}, \mathrm{T}_{1, T D P=5 \%} \mathrm{~T}_{0, T D P}, \xi=0.5 \%$, $\mathrm{C}_{0}=3.41776, \mathrm{~T}=5.5 \mathrm{~s}, \mathrm{U}_{0}=1$ e $\mathrm{V}_{0}=0.48$ 


\section{Equação do modelo de ordem reduzida para o caso T5:}

$$
\begin{aligned}
\ddot{U}+0.025939 \dot{U}+ & {[0.046726-0.003157 \cos (0.432325 \tau)] U } \\
& =0.068868 \cos (0.432325 \tau)+[0.022002+0.006257 \cos (0.432325 \tau)] U^{2} \\
& -0.011693 \dot{U}^{2}-0.719952 U \ddot{U}+[0.003068+0.00083 \cos (0.432325 \tau)] U^{3} \\
& +0.02385 U \dot{U}^{2}-0.193774 U^{2} \ddot{U}-0.000073 U^{4}+0.010328 U^{2} \dot{U}^{2}-0.023099 U^{3} \ddot{U} \\
& -0.000002 U^{5}+0.001021 U^{3} \dot{U}^{2}-0.001028 U^{4} \ddot{U}
\end{aligned}
$$
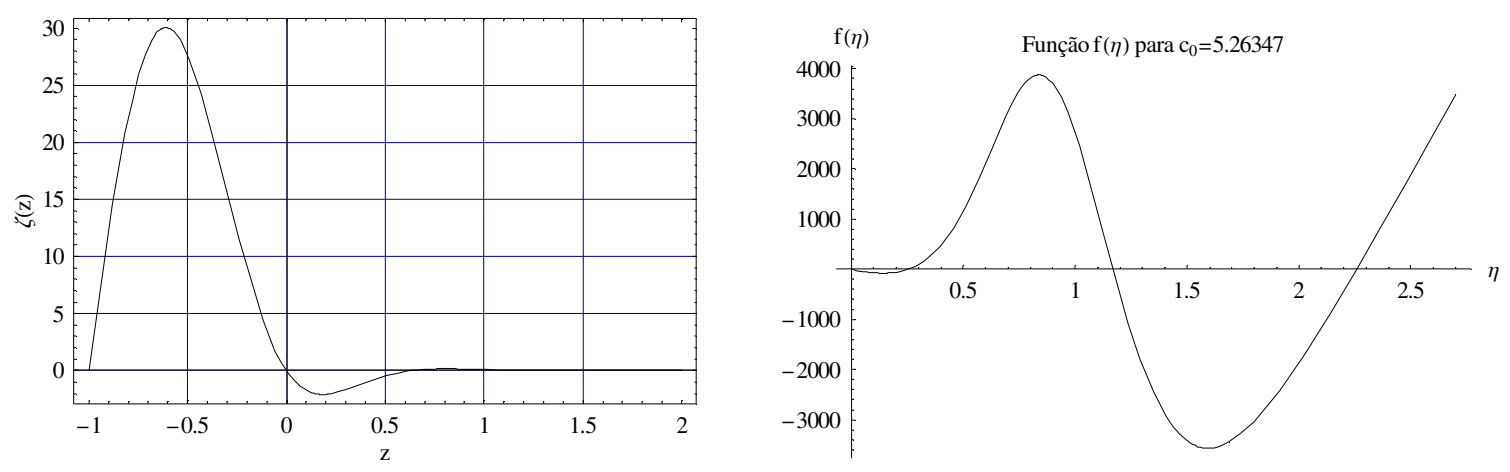

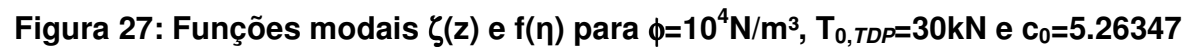
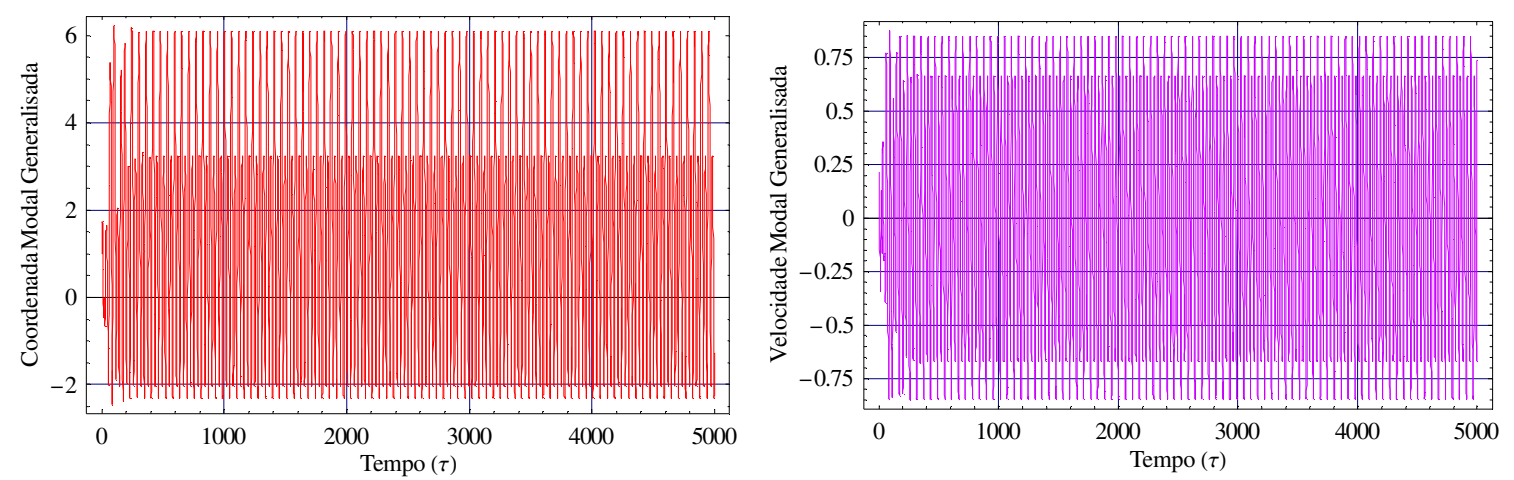

Figura 28: Deslocamento U e velocidade V para $\phi=10^{4} / \mathrm{m}^{3}, \mathrm{~T}_{0, T D P}=40 \mathrm{kN}, \mathrm{T}_{1, T D P=40 \%} \mathrm{~T}_{0, T D P}, \xi=6 \%$, $\mathrm{C}_{0}=5.26347, \mathrm{~T}=3.8 \mathrm{~s}, \mathrm{U}_{0}=1$ e $\mathrm{V}_{0}=0.22$
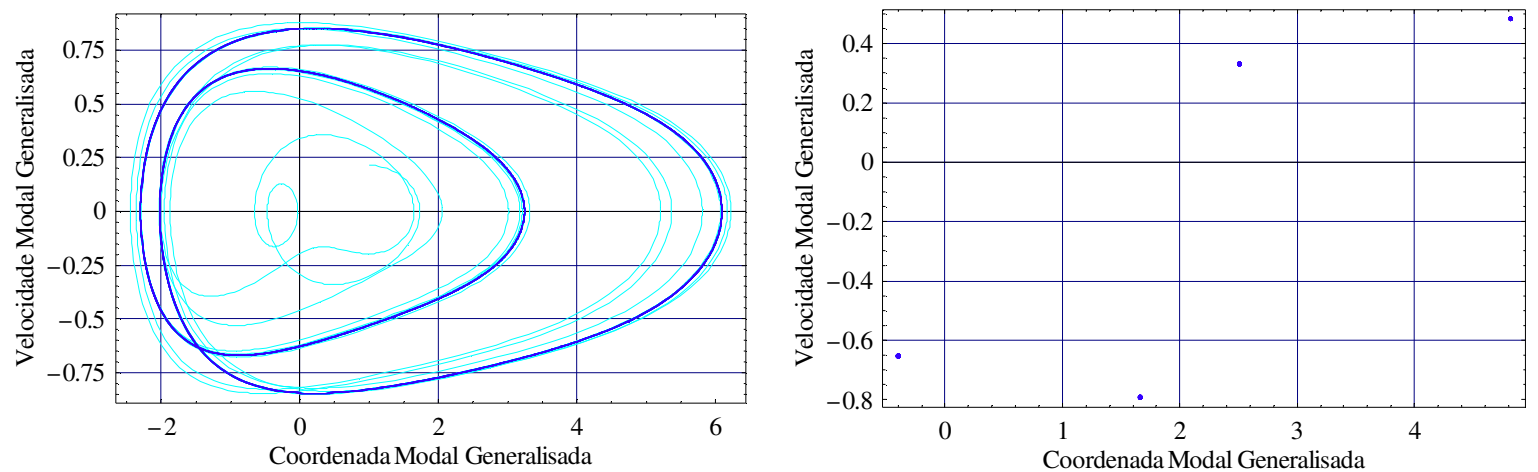

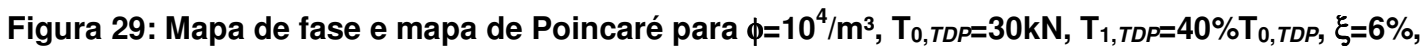
$\mathrm{C}_{0}=5.26347, \mathrm{~T}=3.8 \mathrm{~s}, \mathrm{U}_{0}=1$ e $\mathrm{V}_{0}=0.22$ 
Equação do modelo de ordem reduzida para o caso T6:

$$
\begin{aligned}
\ddot{U}+0.00091 \dot{U}+ & {[0.063944-0.000437 \cos (0.505746 \tau)] U } \\
& =0.015183 \cos (0.505746 \tau)+[0.019763+0.001673 \cos (0.505746 \tau)] U^{2} \\
& -0.011532 \dot{U}^{2}-0.76395 U \ddot{U}+[0.003015+0.00023 \cos (0.505746 \tau)] U^{3} \\
& +0.029273 U \dot{U}^{2}-0.218192 U^{2} \ddot{U}-0.000122 U^{4}+0.013202 U^{2} \dot{U}^{2}-0.027602 U^{3} \ddot{U} \\
& -0.000004 U^{5}+0.001377 U^{3} \dot{U}^{2}-0.001304 U^{4} \ddot{U}
\end{aligned}
$$
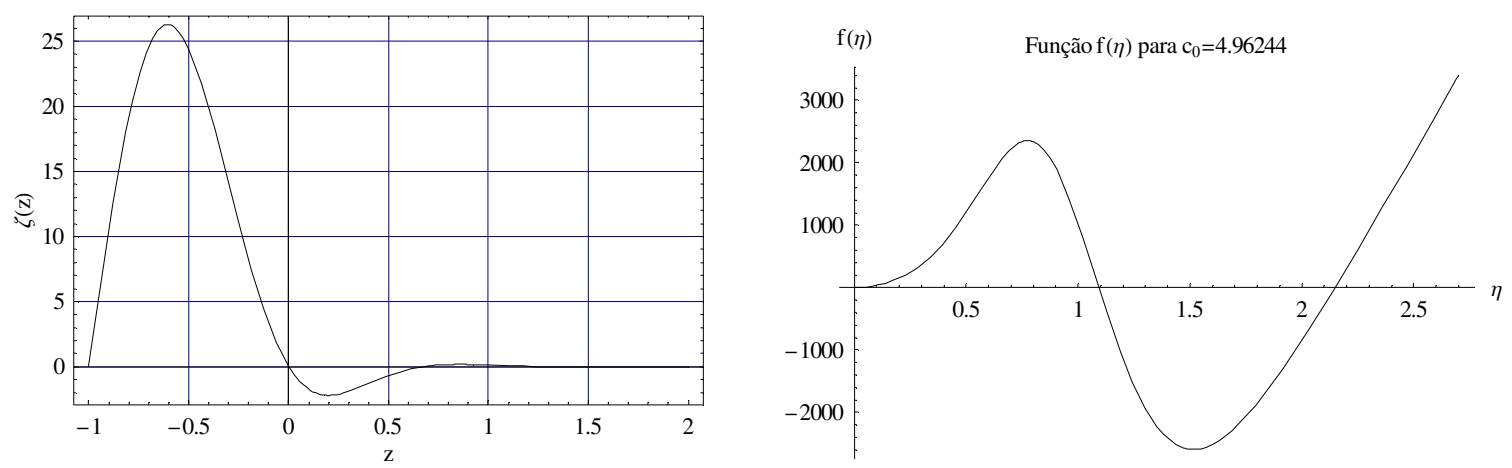

Figura 30: Funções modais $\zeta(z)$ e $f(\eta)$ para $\phi=10^{4} \mathrm{~N} / \mathrm{m}^{3}, T_{0, T D P}=45 \mathrm{kN}$ e $c_{0}=4.96244$
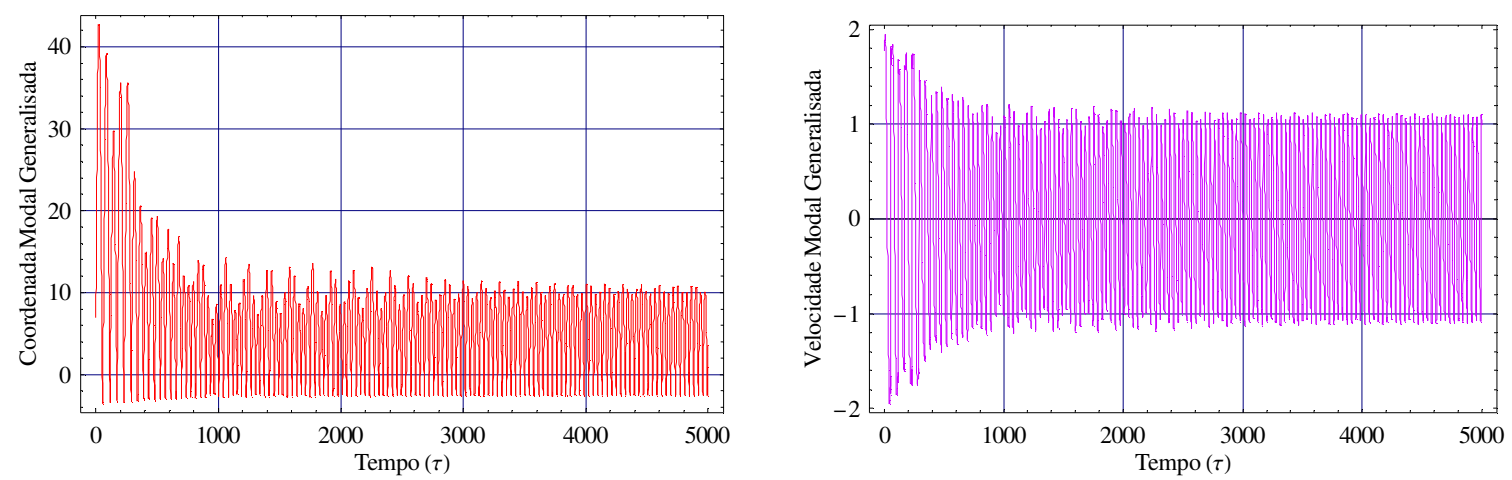

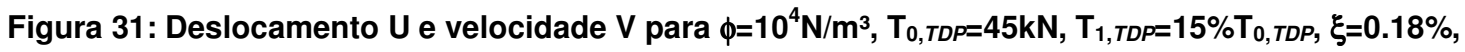
$\mathrm{C}_{0}=4.96244, \mathrm{~T}=3.3 \mathrm{~s}, \mathrm{U}_{0}=7$ e $\mathrm{V}_{0}=1.77$
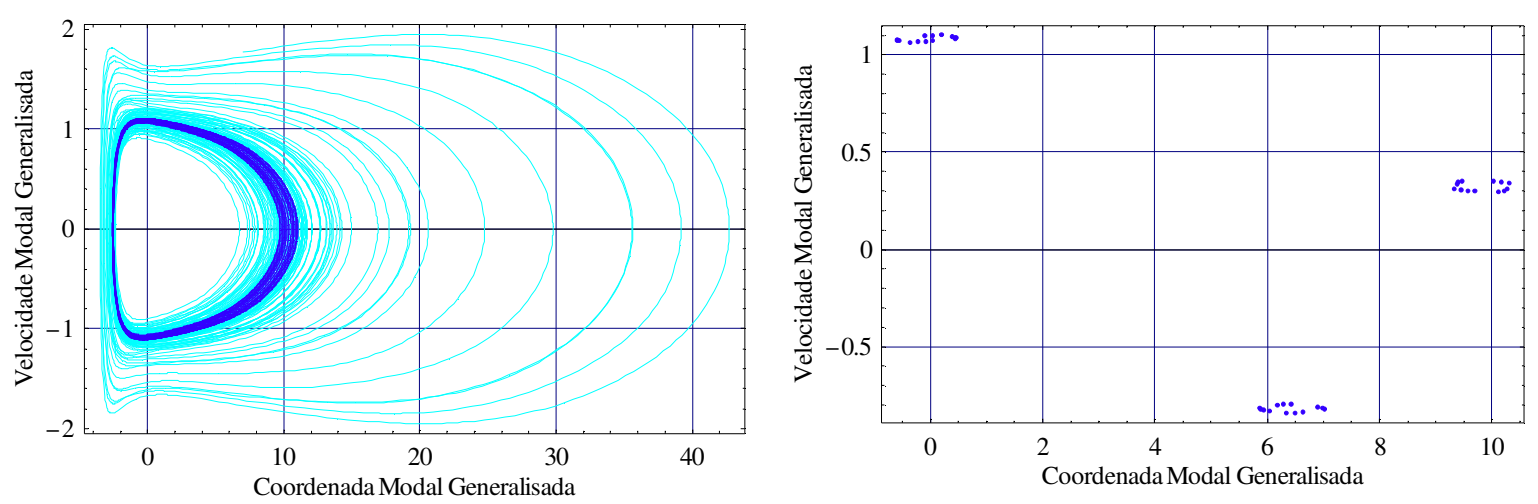

Figura 32: Mapa de fase e mapa de Poincaré para $\phi=104 \mathrm{~N} / \mathrm{m}^{3}, \mathrm{~T} 0, \mathrm{TDP}=45 \mathrm{kN}, \mathrm{T} 1, \mathrm{TDP}=15 \% \mathrm{T0}, \mathrm{TDP}$, $\xi=0.18 \%, c 0=4.96244, \mathrm{~T}=3.3 \mathrm{~s}, \mathrm{U} 0=7$ e V0 $=1.77$ 
Equação do modelo de ordem reduzida para o caso T7:

$\ddot{U}+0.00091 \dot{U}+[0.063944-0.000437 \cos (0.505746 \tau)] U$

$$
\begin{aligned}
& =0.015183 \cos (0.505746 \tau)+[0.019763+0.001673 \cos (0.505746 \tau)] U^{2} \\
& -0.011532 \dot{U}^{2}-0.76395 U \ddot{U}+[0.003015+0.00023 \cos (0.505746 \tau)] U^{3} \\
& +0.029273 U \dot{U}^{2}-0.218192 U^{2} \ddot{U}-0.000122 U^{4}+0.013202 U^{2} \dot{U}^{2}-0.027602 U^{3} \ddot{U} \\
& -0.000003 U^{5}+0.001377 U^{3} \dot{U}^{2}-0.001304 U^{4} \ddot{U}
\end{aligned}
$$
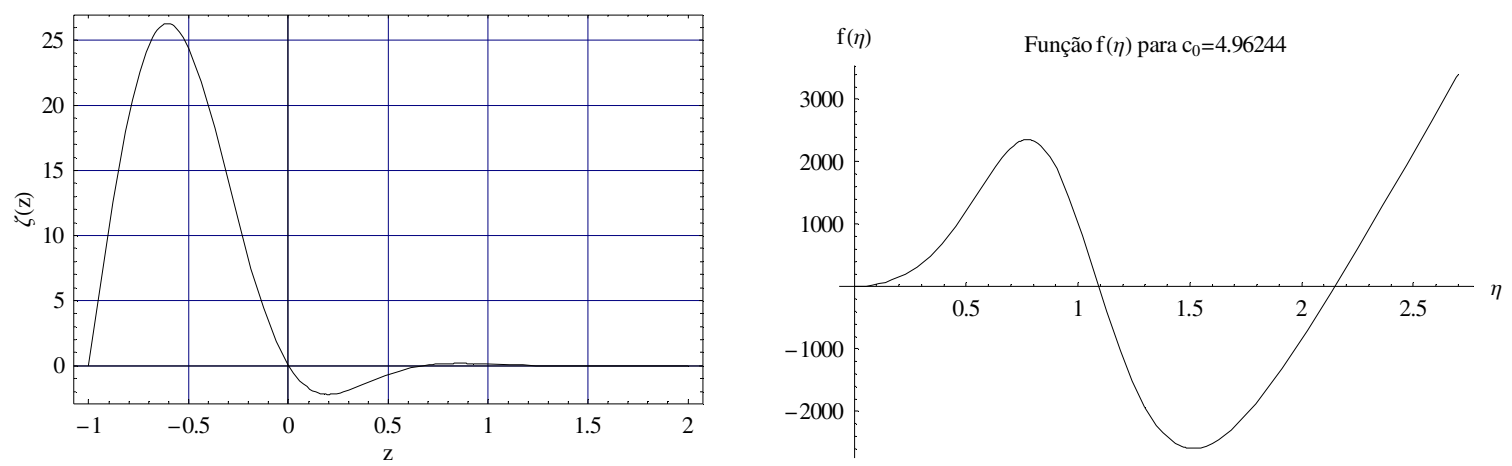

Figura 33: Funções modais $\zeta(z)$ e $f(\eta)$ para $\phi=10^{4} \mathrm{~N} / \mathrm{m}^{3}, T_{0, T D P}=45 \mathrm{kN}$ e $c_{0}=4.96244$
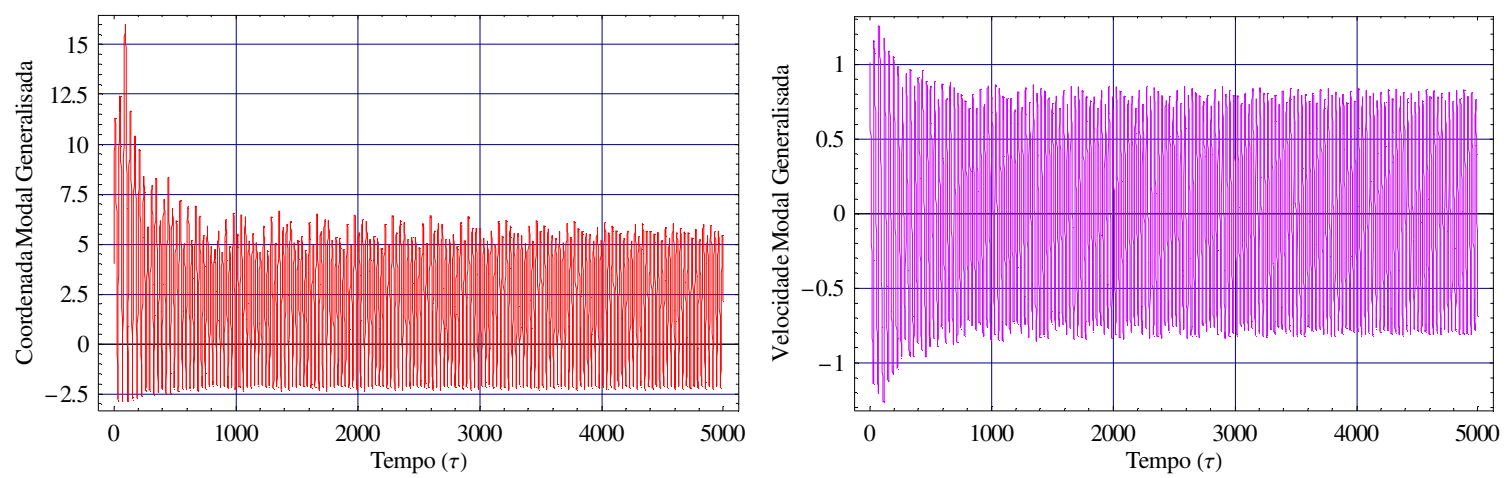

Figura 34: Deslocamento U e velocidade V para $\phi=10^{4} \mathrm{~N} / \mathrm{m}^{3}, \mathrm{~T}_{0, T D P}=45 \mathrm{kN}, \mathrm{T}_{1, T D P}=15 \% \mathrm{~T}_{0, T D P}, \xi=0.18 \%$, $\mathrm{C}_{0}=4.96244, \mathrm{~T}=3.3 \mathrm{~s}, \mathrm{U}_{0}=4$ e $\mathrm{V}_{0}=1.01$
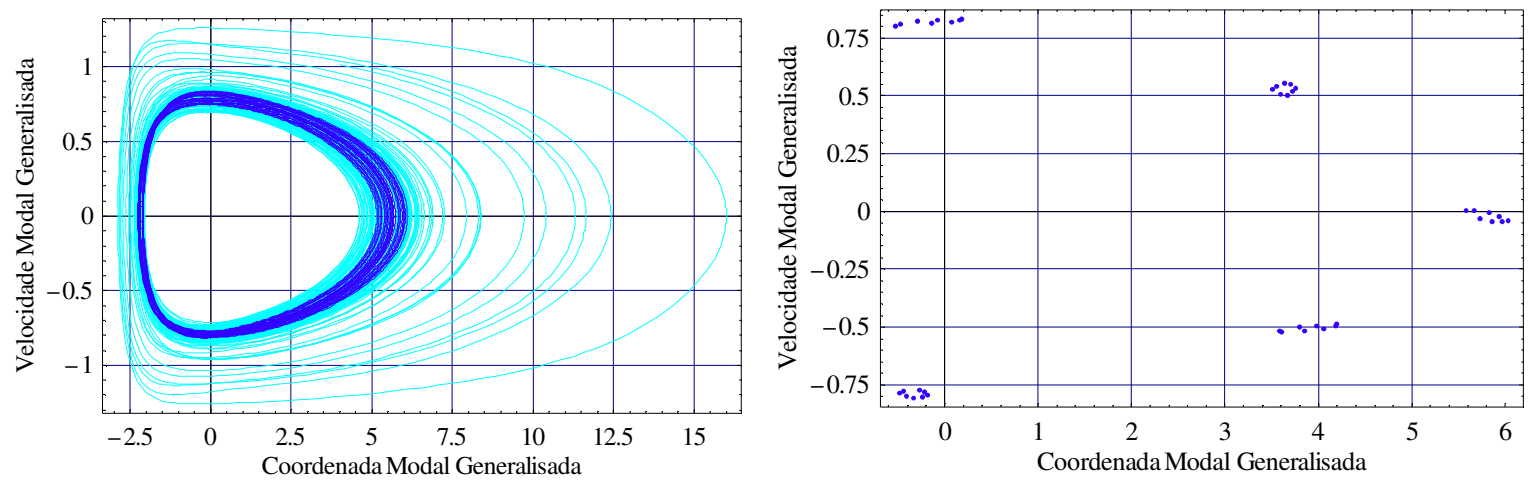

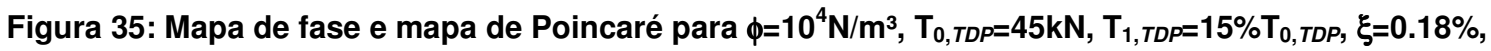
$\mathrm{C}_{0}=4.96244, \mathrm{~T}=3.3 \mathrm{~s}, \mathrm{U}_{0}=4$ e $\mathrm{V}_{0}=1.01$ 
Equação do modelo de ordem reduzida para o caso T8:

$$
\begin{aligned}
\ddot{U}+0.000514 \dot{U}+ & {[0.029392-0.000271 \cos (0.342883 \tau)] U } \\
& =0.003847 \cos (0.342883 \tau)+[0.006358+0.000223 \cos (0.342883 \tau)] U^{2} \\
& -0.011363 \dot{U}^{2}-0.623118 U \ddot{U}+[0.000785+0.000026 \cos (0.342883 \tau)] U^{3} \\
& +0.015211 U \dot{U}^{2}-0.145143 U^{2} \ddot{U}-0.000022 U^{4}+0.005928 U^{2} \dot{U}^{2}-0.014972 U^{3} \ddot{U} \\
& -0.000001 U^{5}+0.000513 U^{3} \dot{U}^{2}-0.000576 U^{4} \ddot{U}
\end{aligned}
$$
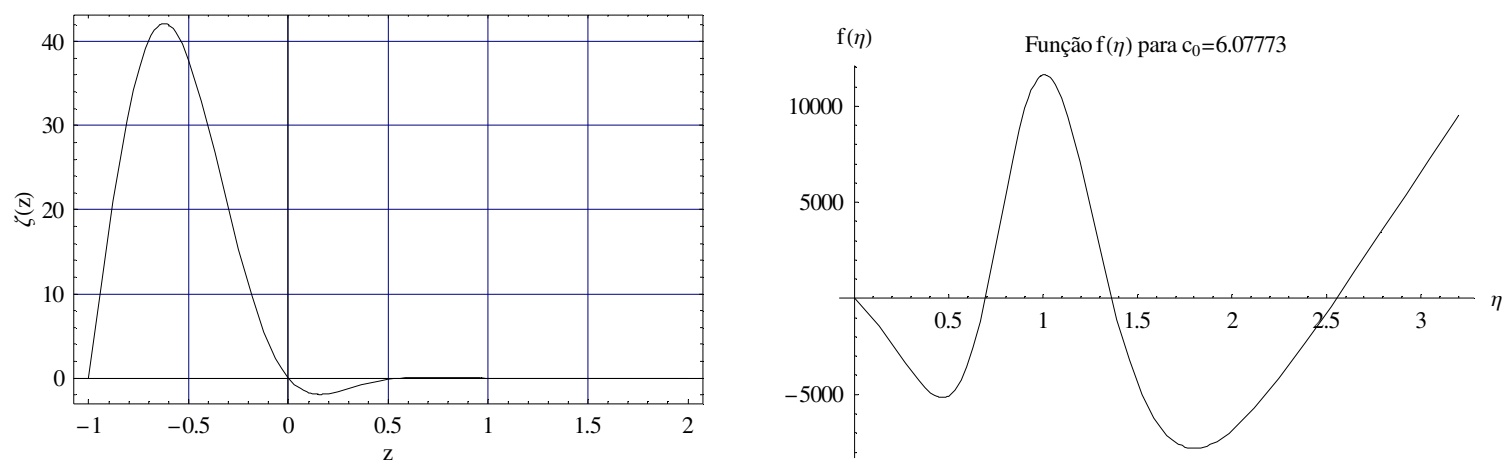

Figura 36: Funções modais $\zeta(z)$ e $f(\eta)$ para $\phi=10^{4} \mathrm{~N} / \mathrm{m}^{3}, \mathrm{~T}_{0, T D P}=30 \mathrm{kN}$ e $c_{0}=6.07773$
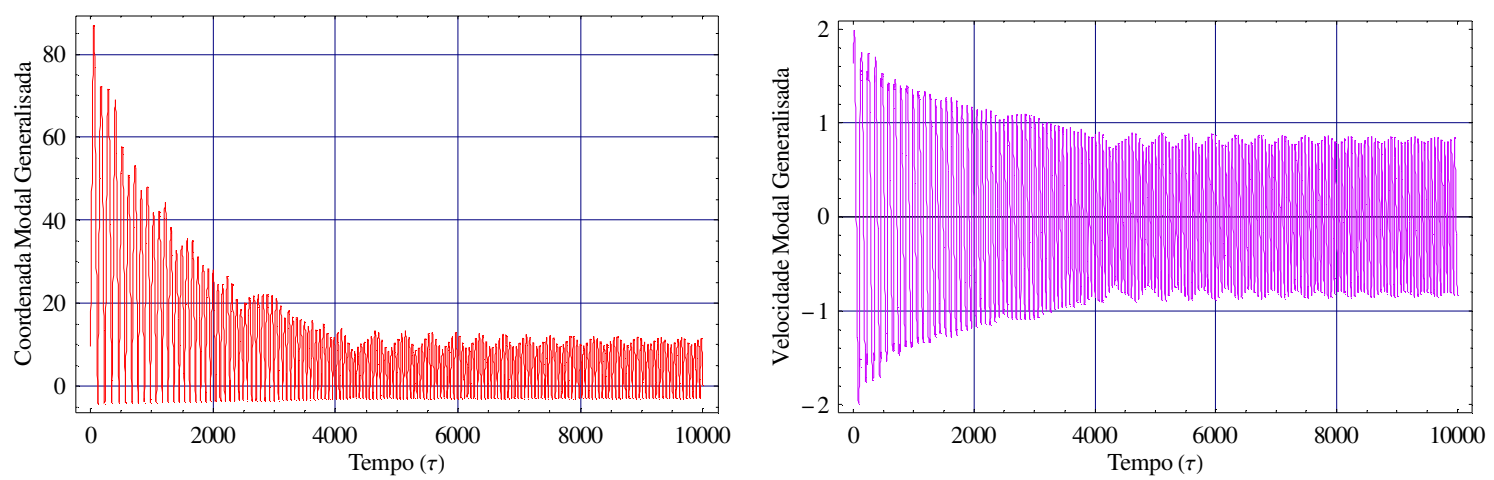

Figura 37: Deslocamento U e velocidade V para $\phi=10^{4} \mathrm{~N} / \mathrm{m}^{3}, \mathrm{~T}_{0, T D P}=30 \mathrm{kN}, \mathrm{T}_{1, T D P}=6.9 \% \mathrm{~T}_{0, T D P}, \xi=0.15 \%$, $\mathrm{C}_{0}=6.07773, \mathrm{~T}=4.8 \mathrm{~s}, \mathrm{U}_{0}=9.5$ e $\mathrm{V}_{0}=1.63$
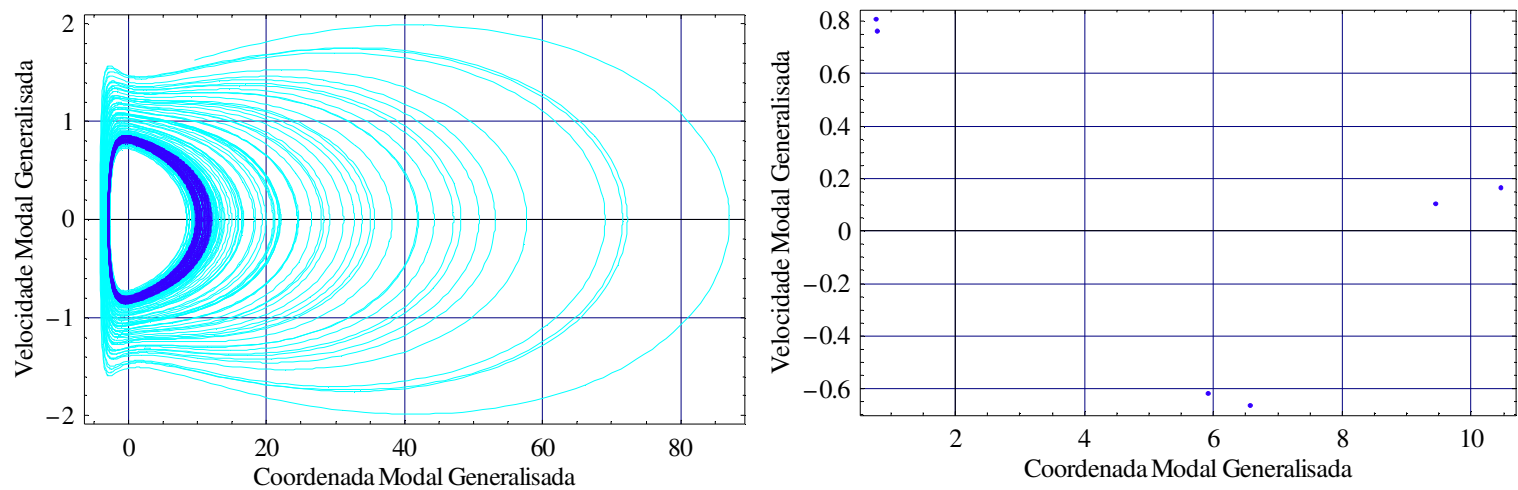

Figura 38: Mapa de fase e mapa de Poincaré para $\phi=10^{4} \mathrm{~N} / \mathrm{m}^{3}, \mathrm{~T}_{0, T D P}=30 \mathrm{kN}, \mathrm{T}_{1, T D P}=6.9 \% \mathrm{~T}_{0, T D P}, \xi=0.15 \%$, $C_{0}=6.07773, T=4.8 \mathrm{~s}, \mathrm{U}_{0}=9.5$ e $\mathrm{V}_{0}=1.63$ 
Equação do modelo de ordem reduzida para o caso T9:

$$
\begin{aligned}
\ddot{U}+0.000514 \dot{U}+ & {[0.029392-0.000271 \cos (0.342883 \tau)] U } \\
& =0.003847 \cos (0.342883 \tau)+[0.006358+0.000223 \cos (0.342883 \tau)] U^{2} \\
& -0.011363 \dot{U}^{2}-0.623118 U \ddot{U}+[0.000785+0.000026 \cos (0.342883 \tau)] U^{3} \\
& +0.015211 U \dot{U}^{2}-0.145143 U^{2} \ddot{U}-0.000022 U^{4}+0.005928 U^{2} \dot{U}^{2}-0.014972 U^{3} \ddot{U} \\
& -0.000001 U^{5}+0.000513 U^{3} \dot{U}^{2}-0.000576 U^{4} \ddot{U}
\end{aligned}
$$
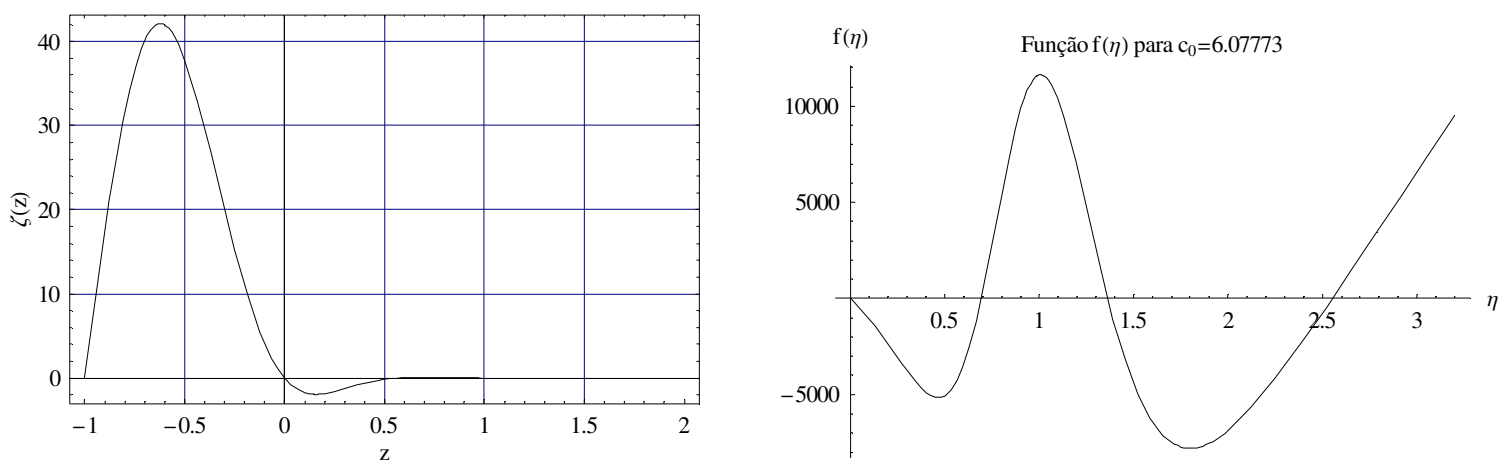

Figura 39: Funções modais $\zeta(z)$ e $f(\eta)$ para $\phi=10^{4} \mathrm{~N} / \mathrm{m}^{3}, \mathrm{~T}_{0, T D P}=30 \mathrm{kN}$ e $c_{0}=6.07773$
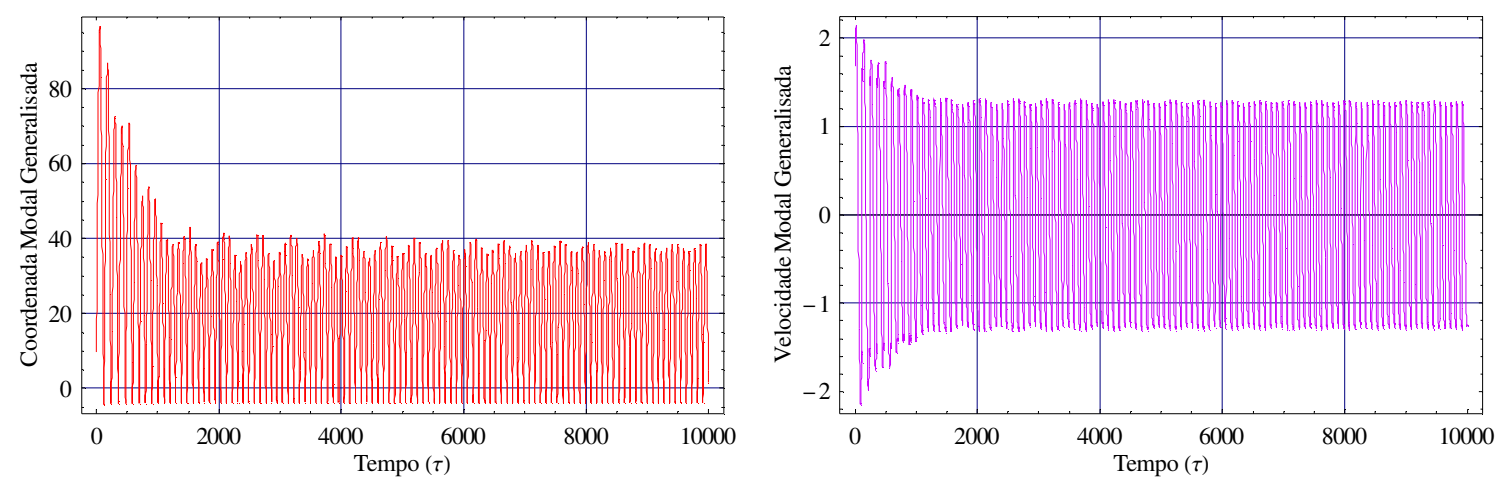

Figura 40: Deslocamento U e velocidade V para $\phi=10^{4} \mathrm{~N} / \mathrm{m}^{3}, \mathrm{~T}_{0, T D P}=30 \mathrm{kN}, \mathrm{T}_{1, T D P}=6.9 \% \mathrm{~T}_{0, T D P}, \xi=0.15 \%$, $\mathrm{C}_{0}=6.07773, \mathrm{~T}=4.8 \mathrm{~s}, \mathrm{U}_{0}=9.8$ e $\mathrm{V}_{0}=1.68$
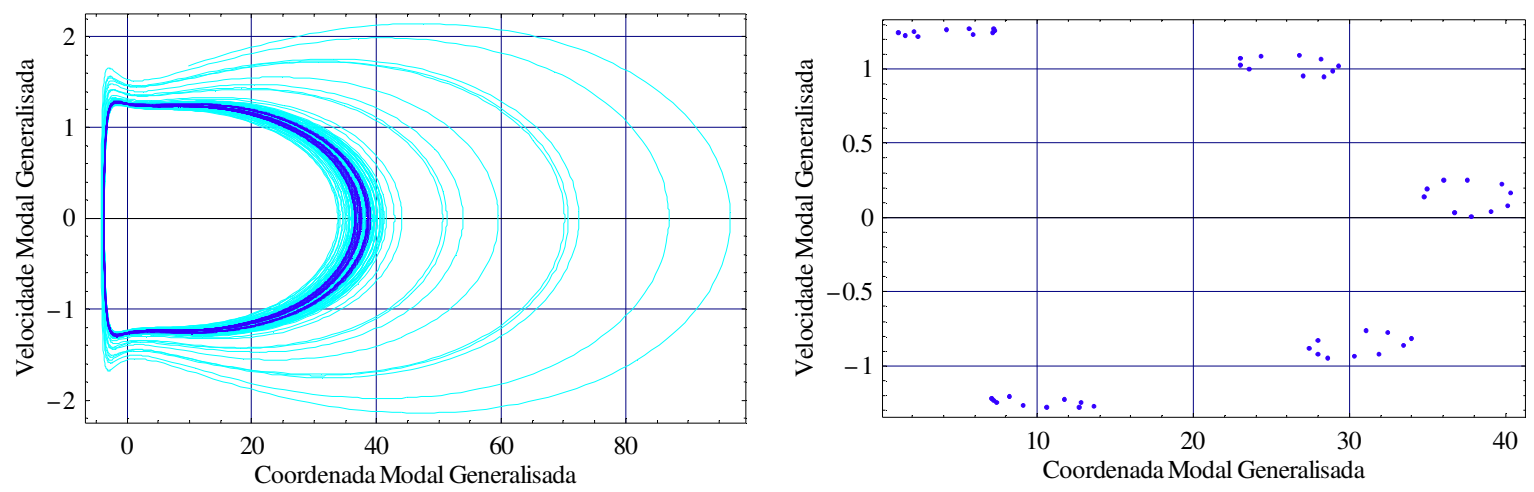

Figura 41: Mapa de fase e mapa de Poincaré para $\phi=10^{4} \mathrm{~N} / \mathrm{m}^{3}, \mathrm{~T}_{0, T D P}=30 \mathrm{kN}, \mathrm{T}_{1, T D P}=6.9 \% \mathrm{~T}_{0, T D P}, \xi=0.15 \%$, $\mathrm{C}_{0}=6.07773, \mathrm{~T}=4.8 \mathrm{~s}, \mathrm{U}_{0}=9.8$ e $\mathrm{V}_{0}=1.68$ 
Equação do modelo de ordem reduzida para o caso T10:

$$
\begin{aligned}
\ddot{U}+0.000514 \dot{U}+ & {[0.029392-0.000271 \cos (0.342883 \tau)] U } \\
& =0.003847 \cos (0.342883 \tau)+[0.006358+0.000223 \cos (0.342883 \tau)] U^{2} \\
& -0.011363 \dot{U}^{2}-0.623118 U \ddot{U}+[0.000785+0.000026 \cos (0.342883 \tau)] U^{3} \\
& +0.015211 U \dot{U}^{2}-0.145143 U^{2} \ddot{U}-0.000022 U^{4}+0.005928 U^{2} \dot{U}^{2}-0.014972 U^{3} \ddot{U} \\
& -0.000001 U^{5}+0.000513 U^{3} \dot{U}^{2}-0.000576 U^{4} \ddot{U}
\end{aligned}
$$
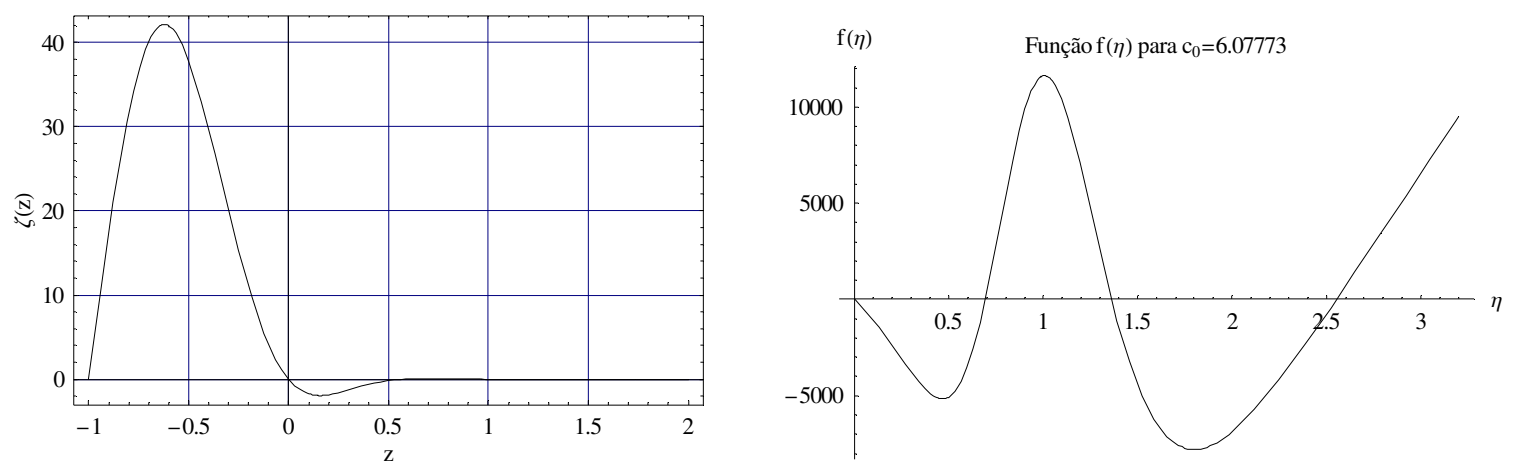

Figura 42: Funções modais $\zeta(z)$ e $f(\eta)$ para $\phi=10^{4} \mathrm{~N} / \mathrm{m}^{3}, T_{0, T D P}=30 \mathrm{kN}$ e $c_{0}=6.07773$
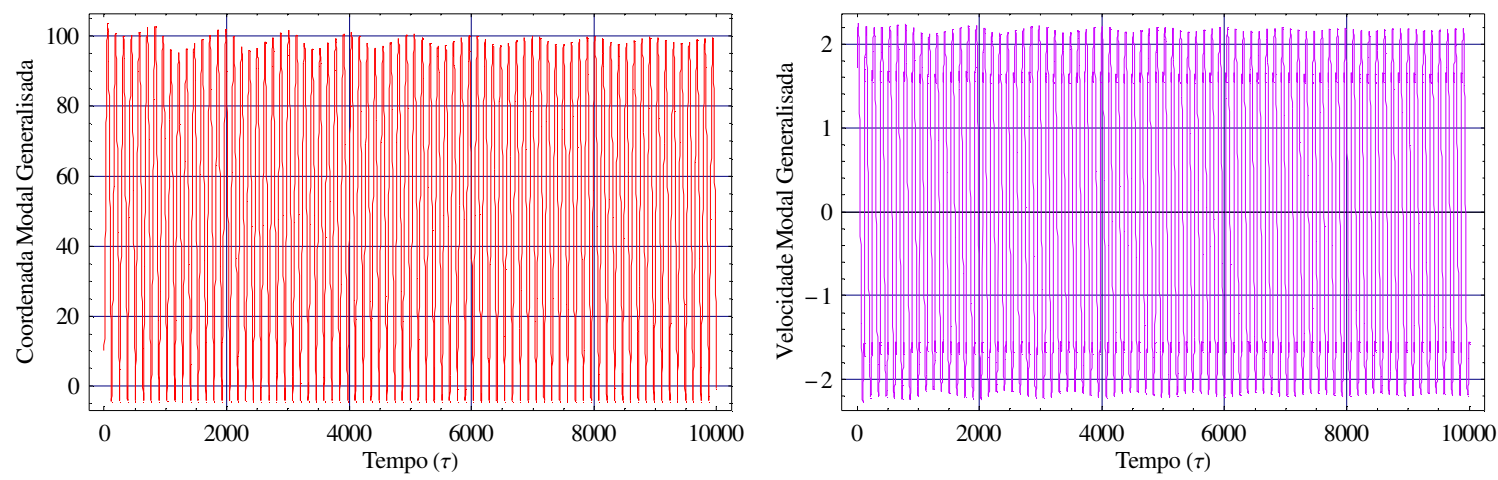

Figura 43: Deslocamento U e velocidade V para $\phi=10^{4} \mathrm{~N} / \mathrm{m}^{3}, \mathrm{~T}_{0, T D P}=30 \mathrm{kN}, \mathrm{T}_{1, T D P}=6.9 \% \mathrm{~T}_{0, T D P}, \xi=0.15 \%$, $c_{0}=6.07773, T=4.8 \mathrm{~s}, \mathrm{U}_{0}=10$ e $\mathrm{V}_{0}=1.71$
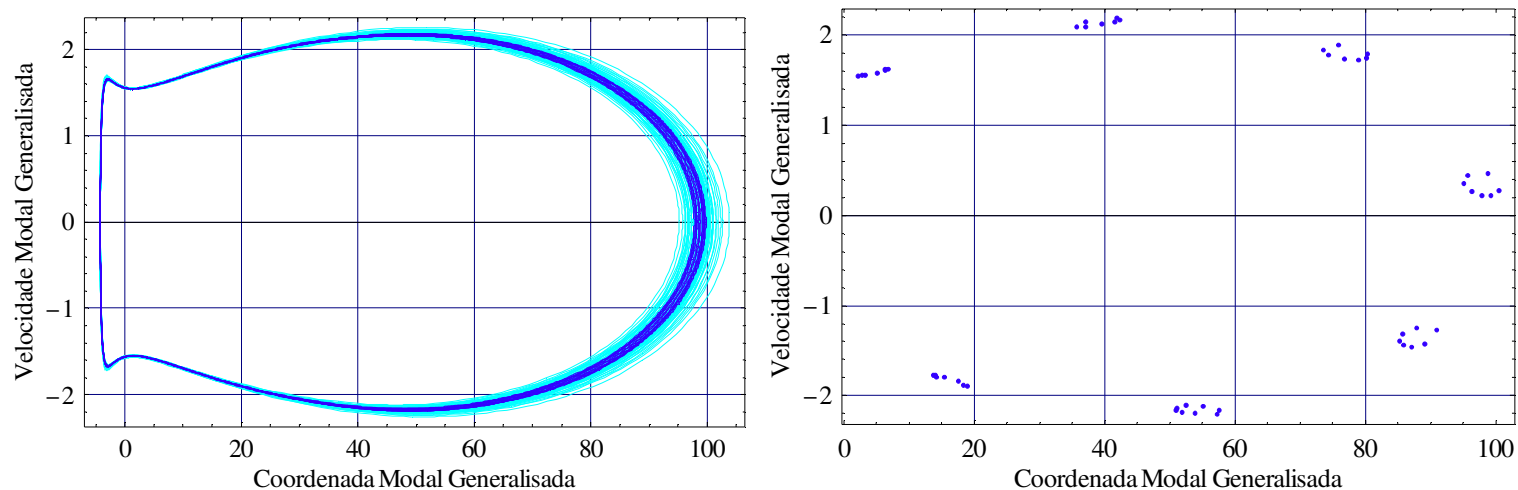

Figura 44: Mapa de fase e mapa de Poincaré para $\phi=10^{4} \mathrm{~N} / \mathrm{m}^{3}, \mathrm{~T}_{0, T D P}=30 \mathrm{kN}, \mathrm{T}_{1, T D P}=6.9 \% \mathrm{~T}_{0, T D P}, \xi=0.15 \%$, $c_{0}=6.07773, T=4.8 s, U_{0}=10$ e $V_{0}=1.71$ 
Equação do modelo de ordem reduzida para o caso T11:

$$
\begin{aligned}
\ddot{U}+0.000514 \dot{U}+ & {[0.029392-0.000271 \cos (0.342883 \tau)] U } \\
& =0.003847 \cos (0.342883 \tau)+[0.006358+0.000223 \cos (0.342883 \tau)] U^{2} \\
& -0.011363 \dot{U}^{2}-0.623118 U \ddot{U}+[0.000785+0.000026 \cos (0.342883 \tau)] U^{3} \\
& +0.015211 U \dot{U}^{2}-0.145143 U^{2} \ddot{U}-0.000022 U^{4}+0.005928 U^{2} \dot{U}^{2}-0.014972 U^{3} \ddot{U} \\
& -0.000001 U^{5}+0.000513 U^{3} \dot{U}^{2}-0.000576 U^{4} \ddot{U}
\end{aligned}
$$
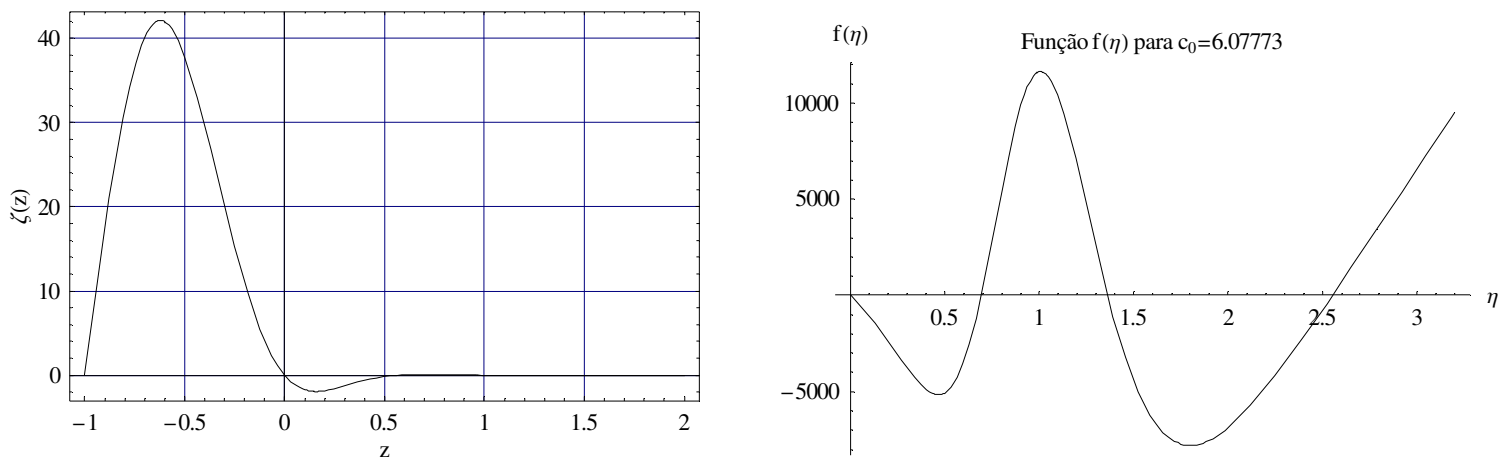

Figura 45: Funções modais $\zeta(z)$ e $f(\eta)$ para $\phi=10^{4} \mathrm{~N} / \mathrm{m}^{3}, \mathrm{~T}_{0, T D P}=30 \mathrm{kN}$ e $c_{0}=6.07773$
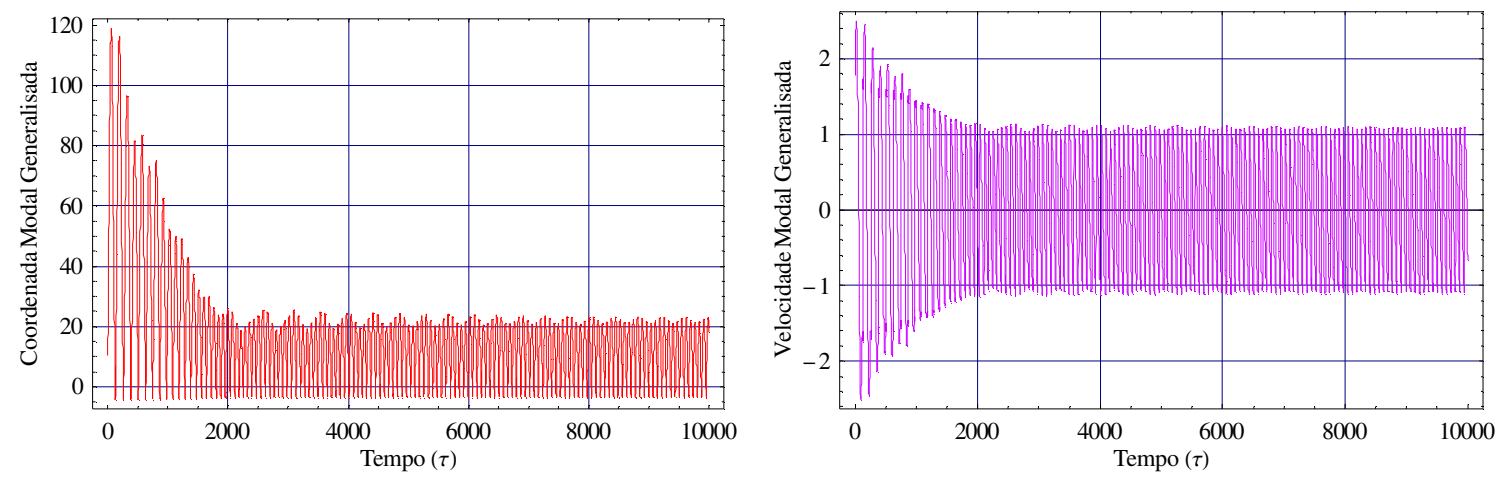

Figura 46: Deslocamento U e velocidade V para $\phi=10^{4} \mathrm{~N} / \mathrm{m}^{3}, \mathrm{~T}_{0, T D P}=30 \mathrm{kN}, \mathrm{T}_{1, T D P}=6.9 \% \mathrm{~T}_{0, T D P}, \xi=0.15 \%$, $\mathrm{C}_{0}=6.07773, \mathrm{~T}=4.8 \mathrm{~s}, \mathrm{U}_{0}=10.4$ e $\mathrm{V}_{0}=1.78$
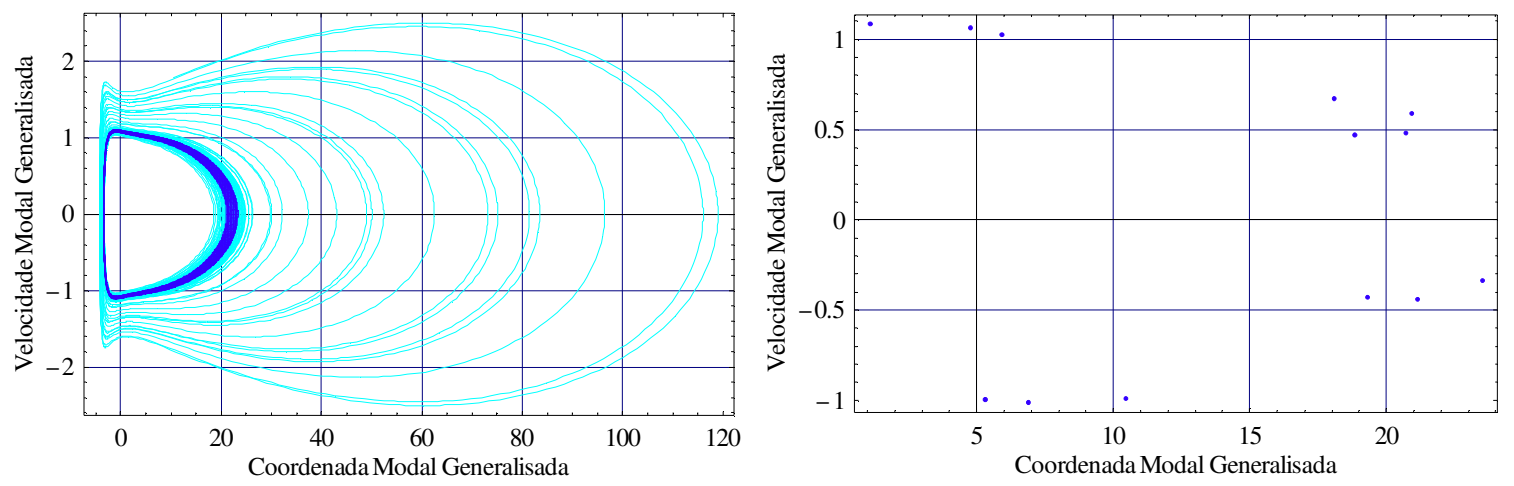

Figura 47: Mapa de fase e mapa de Poincaré para $\phi=10^{4} \mathrm{~N} / \mathrm{m}^{3}, \mathrm{~T}_{0, T D P}=30 \mathrm{kN}, \mathrm{T}_{1, T D P=6.9 \%} \mathrm{~T}_{0, T D P}, \quad \xi=0.15 \%$, $\mathrm{C}_{0}=6.07773, \mathrm{~T}=4.8 \mathrm{~s}, \mathrm{U}_{0}=10.4$ e $\mathrm{V}_{0}=1.78$ 
O estudo de caso com os diferentes processamentos descritos acima podem ser resumidos na Tab. 4 a seguir. Os valores para período referem-se ao período associado à freqüência excitante considerado no processamento:

\begin{tabular}{|c|c|c|c|c|c|c|c|c|c|c|c|}
\hline Caso & $T 1$ & $T 2$ & T3 & $T 4$ & T5 & T6 & $T 7$ & $T 8$ & T9 & T10 & $T 11$ \\
\hline$\varphi_{i}\left(N / m^{3}\right)$ & $10^{2}$ & $10^{2}$ & $10^{3}$ & $10^{3}$ & $10^{4}$ & $10^{4}$ & $10^{4}$ & $10^{4}$ & $10^{4}$ & $10^{4}$ & $10^{4}$ \\
\hline$T_{0, T D P}(k N)$ & 10 & 10 & 25 & 30 & 40 & 45 & 45 & 30 & 30 & 30 & 30 \\
\hline$c_{0, i}$ & 3.3 & 3.3 & 3.7 & 3.4 & 5.3 & 5.0 & 5.0 & 6.1 & 6.1 & 6.1 & 6.1 \\
\hline$\omega_{0, i}$ & 0.47 & 0.47 & 0.37 & 0.48 & 0.22 & 0.25 & 0.25 & 0.17 & 0.17 & 0.17 & 0.17 \\
\hline$\Omega_{0, i}$ & 0.94 & 0.94 & 0.74 & 0.95 & 0.43 & 0.51 & 0.51 & 0.34 & 0.34 & 0.34 & 0.34 \\
\hline$T_{0, i}$ & 6.66 & 6.66 & 8.48 & 6.61 & 14.53 & 12.42 & 12.42 & 18.32 & 18.32 & 18.32 & 18.32 \\
\hline Período (s) & 17.6 & 17.6 & 7.1 & 5.5 & 3.8 & 3.3 & 3.3 & 4.8 & 4.8 & 4.8 & 4.8 \\
\hline$\xi(\%)$ & 0 & 0 & 1 & 0.5 & 6 & 0.18 & 0.18 & 0.15 & 0.15 & 0.15 & 0.15 \\
\hline$U_{0}$ & 0.5 & 2.0 & 1.0 & 1.0 & 1.0 & 7.0 & 4.0 & 9.5 & 9.8 & 10.0 & 10.4 \\
\hline$v_{0}$ & 0.24 & 0.94 & 0.37 & 0.48 & 0.22 & 1.77 & 1.01 & 1.63 & 1.68 & 1.71 & 1.78 \\
\hline$U_{\text {estacionário }}$ & 1.6 & 6.0 & 3.0 & 1.3 & 6.0 & 10.0 & 6.0 & 10.0 & 35.0 & 95.0 & 20.0 \\
\hline$V_{\text {estacionário }}$ & 0.5 & 1.0 & 0.8 & 0.6 & 0.8 & 1.2 & 0.8 & 0.9 & 1.2 & 2.1 & 1.2 \\
\hline$U_{\max }$ & 1.6 & 6.0 & 5.0 & 2.0 & 6.0 & 42.0 & 16.0 & 85.0 & 95.0 & 100.0 & 120.0 \\
\hline$V_{\max }$ & 0.5 & 1.0 & 1.0 & 0.8 & 0.8 & 2.0 & 1.2 & 2.0 & 2.1 & 2.2 & 2.3 \\
\hline
\end{tabular}

Tabela 4: Resumo dos processamentos na abordagem teórica do estudo de caso

Note-se que os casos de T1 a T7 diferem entre si quanto aos parâmetros de entrada (coeficiente de rigidez do solo $\varphi$, tração estática $T_{0, T D P}$ no TDP e taxa de amortecimento $\xi$ ), ou em relação às condições iniciais. Já os casos de T8 a T11 variam apenas em relação às condições iniciais na integração do modelo de ordem reduzida.

Observa-se nos casos de T8 a T11 que há indícios de diferentes atratores para um mesmo oscilador harmônico (vide Fig. 48 abaixo), uma vez que, a partir de diferentes condições iniciais, chega-se a distintas soluções periódicas. Neste contexto, pode-se citar o trabalho desenvolvido em Gonçalves et al [18], e a possibilidade de abordagem do problema quanto à integridade de bacias de atração. Além disso, pode-se notar que para condições iniciais maiores (caso T10, por exemplo), a capacidade de ampliação do deslocamento pode chegar a 
aproximadamente 10 vezes, e da velocidade a 1.5 vezes. Abaixo, os mapas de fase dos casos T8 a T11:
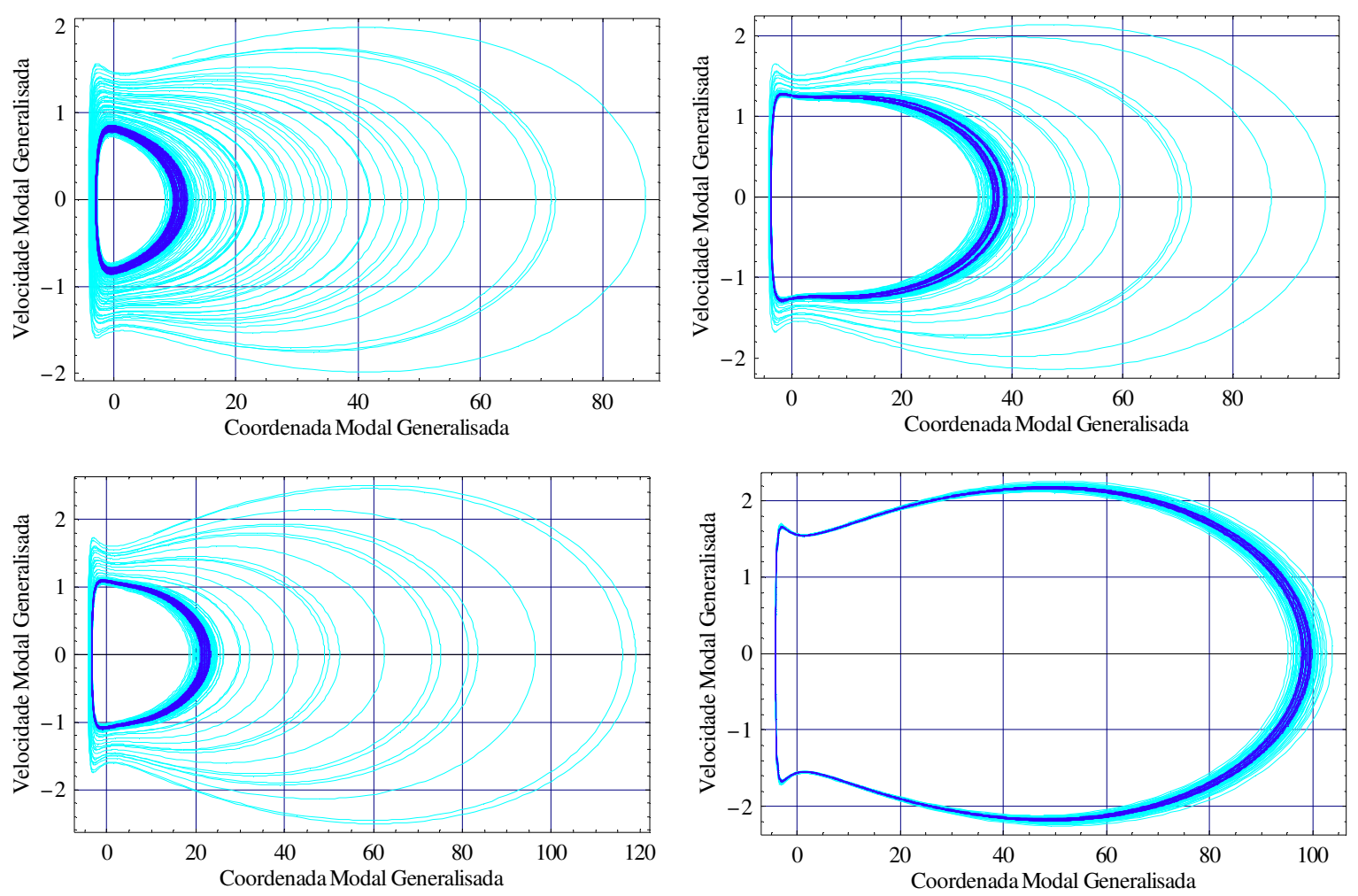

Figura 48: Mapas de fase para $\phi=10^{4} \mathrm{~N} / \mathrm{m}^{3}, \mathrm{~T}_{0, T D P=30 \mathrm{kN}}, \mathrm{T}_{1, T D P=6.9 \%} \mathrm{~T}_{0, T D P}, \quad \xi=0.15 \%, c_{0}=6.07773, \mathrm{~T}=4.8 \mathrm{~s}$, para as seguintes condições iniciais (em sentido horário, a partir do quadrante superior esquerdo):

$\mathrm{U}_{0}=9.5$ e $\mathrm{V}_{0}=1.63, \mathrm{U}_{0}=9.8$ e $\mathrm{V}_{0}=1.68, \mathrm{U}_{0}=10$ e $\mathrm{V}_{0}=1.71$, e $\mathrm{U}_{0}=10.4$ e $\mathrm{V}_{0}=1.78$

Nota-se também a assimetria do diagrama de fase com relação aos deslocamentos, como no caso da Fig. 44, por exemplo. Nela, os valores negativos de deslocamento são pequenos, e há uma abrupta mudança da velocidade, o que caracteriza comportamento similar ao caso de impacto. Tal comportamento pode ser observado nos casos em que há baixa taxa de amortecimento ( $\xi \leq 0.5 \%$ ). Para casos em que a taxa de amortecimento é maior este tipo de fenômeno praticamente desaparece.

Note-se no caso T1 indícios de "traveling wave", que pode ser verificado com a forma do histórico do deslocamento na forma de pulsos. 
$\mathrm{Na}$ Tab. 5 abaixo podemos avaliar a freqüência obtida no modelo com flexão simples e no modelo de ordem reduzida, com flexão composta:

\begin{tabular}{|c|c|c|c|c|c|c|c|c|c|c|c|}
\hline Caso & $T 1$ & $T 2$ & $T 3$ & T4 & $T 5$ & $T 6$ & $T 7$ & $T 8$ & $T 9$ & $T 10$ & $T 11$ \\
\hline$\varphi_{i}\left(N / m^{3}\right)$ & $10^{2}$ & $10^{2}$ & $10^{3}$ & $10^{3}$ & $10^{4}$ & $10^{4}$ & $10^{4}$ & $10^{4}$ & $10^{4}$ & $10^{4}$ & $10^{4}$ \\
\hline$T_{0, T D P}(k N)$ & 10 & 10 & 25 & 30 & 40 & 45 & 45 & 30 & 30 & 30 & 30 \\
\hline$c_{0, i}$ & 3.33 & 3.33 & 3.74 & 3.42 & 5.26 & 4.96 & 4.96 & 6.08 & 6.08 & 6.08 & 6.08 \\
\hline$\omega_{\text {FLEXÃO SIMPLES }}$ & 0.97 & 0.97 & 0.90 & 0.96 & 0.59 & 0.64 & 0.64 & 0.47 & 0.47 & 0.47 & 0.47 \\
\hline$\omega_{\text {FLEXÃO COMPOSTA }}$ & 0.47 & 0.47 & 0.37 & 0.48 & 0.22 & 0.25 & 0.25 & 0.17 & 0.17 & 0.17 & 0.17 \\
\hline $\boldsymbol{R}_{\text {elação entre frequências }}$ & 0.48 & 0.48 & 0.41 & 0.49 & 0.37 & 0.39 & 0.39 & 0.36 & 0.36 & 0.36 & 0.36 \\
\hline
\end{tabular}

Tabela 5: Tabela de comparação entre freqüência adimensional do modelo original e freqüência adicional do modelo de ordem reduzida

Note-se que a relação entre as freqüências naturais para os casos de flexão simples e composta deve ser compreendida à luz do efeito combinado da rigidez do solo $\varphi$ e da tração estática $T_{0, T D P}$ no TDP. No estudo de caso acima, verifica-se freqüência natural no modelo com flexão composta entre metade e um terço em relação à freqüência obtida no modelo com flexão simples.

Pode-se observar, nos mapas de Poincaré, diferentes possibilidades de comportamento dinâmico. Nos casos T1 e T2 há o comportamento típico de uma solução quase periódica, que geralmente surge após uma bifurcação de Hopf da solução harmônica. Nos casos T3 e T4 a solução tem período igual ao dobro do período da força excitante, que surge após bifurcação por duplicação de período (period-doubling ou flip bifurcation). No caso T5 a solução tem período quatro, bem definido. No caso T6 a solução tem período três, com um pequeno efeito do transiente, o que geralmente indica a proximidade de um regime caótico. Nos casos T7 a T11 observa-se pequeno efeito do transiente, similar ao que ocorre no caso T6, com períodos cinco, no caso T7, três, em T8, cinco, em T9, sete, em T10, e quatro, em T11. 


\subsection{Aplicação à análise dinâmica local na região do TDP em risers flexíveis em catenária para condições de movimento imposto no topo (heave / surge)}

Uma vez desenvolvido o modelo para o oscilador harmônico forçado nãolinear, e após verificar na seção 4.1. a sensibilidade e o comportamento frente a diferentes parâmetros e condições iniciais, pretende-se aqui associar o modelo de viga esbelta sob flexão composta com contato unilateral em meio elástico ao estudo da dinâmica local (nas proximidades do TDP) de risers flexíveis em catenária (SCR).

Para o estudo da dinâmica global do caso prático, utilizou-se a ferramenta de processamento Poliflex3D, versão 2.0.1, Martins et al, [34]. O anexo 7.4. ilustra alguns dos parâmetros de entrada utilizados e algumas das respostas obtidas para tração estática e dinâmica no $T D P$, respectivamente $T_{0, T D P}$ e $T_{1, T D P}$. A faixa para tração estática no $T D P$, de $20 \mathrm{kN}$ a $50 \mathrm{kN}$, mostrou-se compatível com a realidade para algumas configurações de riser, particularmente para as profundidades em torno de $200 \mathrm{~m}$ e $300 \mathrm{~m}$. O coeficiente de rigidez do solo adotado foi de $\varphi=10^{4} \mathrm{~N} / \mathrm{m}$ e $\varphi=10^{5} \mathrm{~N} / \mathrm{m}$. As condições iniciais também foram inspiradas do programa Poliflex $3 \mathrm{D}$, onde os resultados dos processamentos revelaram que podem ser adotados valores da ordem de $0.1 \mathrm{~m}$ como deslocamento inicial para o TDP.

Os processamentos foram feitos tendo como base algumas propriedades do riser. A tabela Tab. 6 abaixo resume as propriedades consideradas: 


\begin{tabular}{|l|c|}
\hline Módulo de Young & $E=2.1 \times 10^{11} \mathrm{~N} / \mathrm{m}^{2}$ \\
\hline Diâmetro nominal & $D=0.2032 \mathrm{~m}$ \\
\hline Área da Seção Transversal & $A=1.1021 \times 10^{-2} \mathrm{~m}^{2}$ \\
\hline Momento de Inércia & $I=4.72143 \times 10^{-5} \mathrm{~m}^{4}$ \\
\hline Massa por unidade de comprimento (incluindo a massa adicional) & $\rho=141.24 \mathrm{~kg} / \mathrm{m}$ \\
\hline Peso próprio submerso do riser por unidade de comprimento & $p=727 \mathrm{~N} / \mathrm{m}$ \\
\hline
\end{tabular}

Tabela 6: Propriedades físicas do riser considerado no estudo de caso de análise paramétrica, ver Pesce et al [28]

Faz-se uso novamente dos modos de vibração obtidos no modelo com flexão simples, como dado de entrada do problema: 


\begin{tabular}{|c|c|c|c|c|}
\hline$\varphi\left(N / m^{3}\right)$ & $T_{0, T D P}(k N)$ & $c_{0}$ & $\eta=\sqrt[4]{\frac{1-\omega^{2}}{\omega^{2}}}$ & $\boldsymbol{\omega}$ \\
\hline \multirow{4}{*}{$10^{5}$} & \multirow{4}{*}{50} & \multirow{4}{*}{8.37176} & 3.37955 & 0.0872218 \\
\hline & & & 1.85700 & 0.278511 \\
\hline & & & 1.20792 & 0.565332 \\
\hline & & & 0.681808 & 0.906809 * \\
\hline \multirow{4}{*}{$10^{5}$} & \multirow{4}{*}{40} & \multirow{4}{*}{9.35991} & 3.73516 & 0.0714937 \\
\hline & & & 3.73516 & 0.229196 \\
\hline & & & 2.06081 & $0.469117^{*}$ \\
\hline & & & 0.905335 & 0.773407 \\
\hline \multirow{2}{*}{$10^{4}$} & \multirow{2}{*}{50} & \multirow{2}{*}{4.70779} & 2.0567 & 0.230064 \\
\hline & & & 1.023921 & 0.690202 * \\
\hline \multirow{3}{*}{$10^{4}$} & \multirow{3}{*}{40} & \multirow{3}{*}{5.26347} & 2.25856 & 0.192375 \\
\hline & & & 1.16997 & 0.589902 * \\
\hline & & & 0.2544522 & 0.997911 \\
\hline \multirow{3}{*}{$10^{4}$} & \multirow{3}{*}{30} & \multirow{3}{*}{6.07773} & 2.55303 & 0.151648 \\
\hline & & & 1.36323 & 0.473853 \\
\hline & & & 0.695264 & 0.900328 * \\
\hline \multirow{4}{*}{$10^{4}$} & \multirow{4}{*}{20} & \multirow{4}{*}{7.44366} & 3.04542 & 0.1072 \\
\hline & & & 1.66219 & 0.340337 \\
\hline & & & 1.03636 & 0.68143 * \\
\hline & & & 0.216601 & 0.998901 \\
\hline
\end{tabular}

Tabela 7: Modos de vibração obtidos para viga sob flexão simples

Os modos marcados com $\left(^{*}\right)$ mostram quais os modos foram utilizados nos processamentos, nos casos em que, para um determinado valor de $c_{0}$, há mais de um modo de vibração. O motivo de se utilizar os segundos modos de vibração aos primeiros modos foi o fato de não se ter encontrado casos em que fosse possível observar ressonância paramétrica clássica. 
Dada a disparidade entre os valores encontrados na literatura para o valor a ser considerado como taxa de amortecimento equivalente $\xi$, que representa tanto o amortecimento estrutural quanto o fluido-dinâmico, utilizou-se para os cinco primeiros processamentos, P1 a P6, taxa de amortecimento $\xi=10 \%$, conforme Aranha e Pinto [1], e para os cinco últimos casos, P7 a P12, taxa de amortecimento de $\xi=30 \%$, conforme Sanches [31]. 


\section{Equação do modelo de ordem reduzida para o caso P1:}

$$
\begin{aligned}
\ddot{U}+0.016282 \dot{U} & +[0.006628-0.002163 \cos (0.162829 t)] U \\
& =0.013551 \cos (0.162829 t)+[0.002259+0.000152 \cos (0.162829 t)] U^{2} \\
& -0.027649 \dot{U}^{2}-0.50514 U \ddot{U}+[0.000308+0.000026 \cos (0.162829 t)] U^{3} \\
& +0.020408 U \dot{U}^{2}-0.095297 U^{2} \ddot{U}-0.00001 U^{4}+0.00698 U^{2} \dot{U}^{2}-0.007953 U^{3} \ddot{U} \\
& -0.000001 U^{5}+0.000502 U^{3} \dot{U}^{2}-0.000247 U^{4} \ddot{U}
\end{aligned}
$$
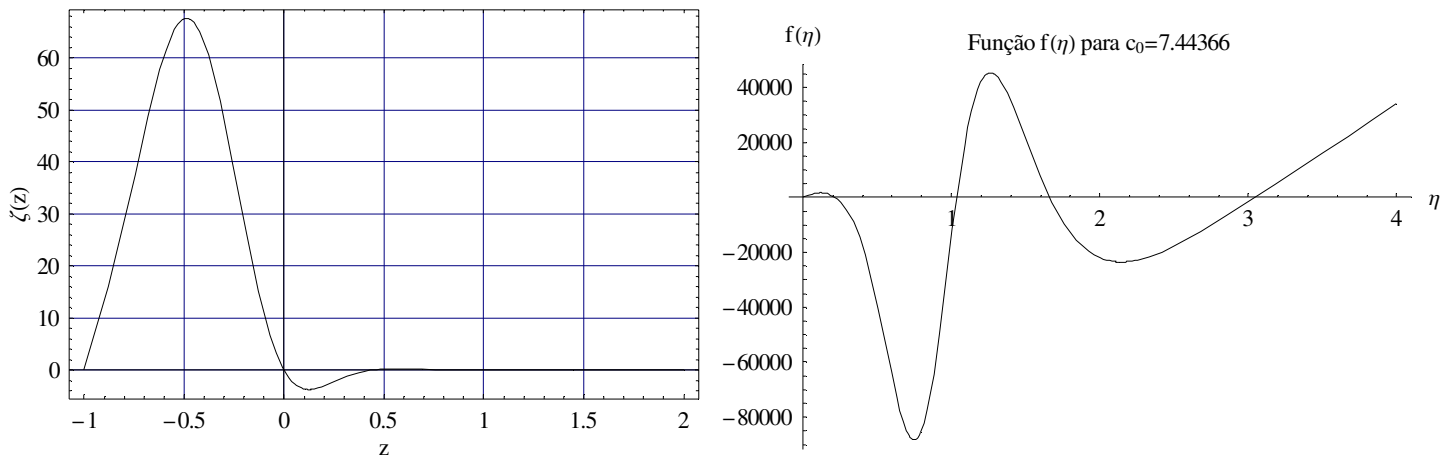

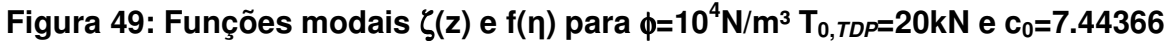
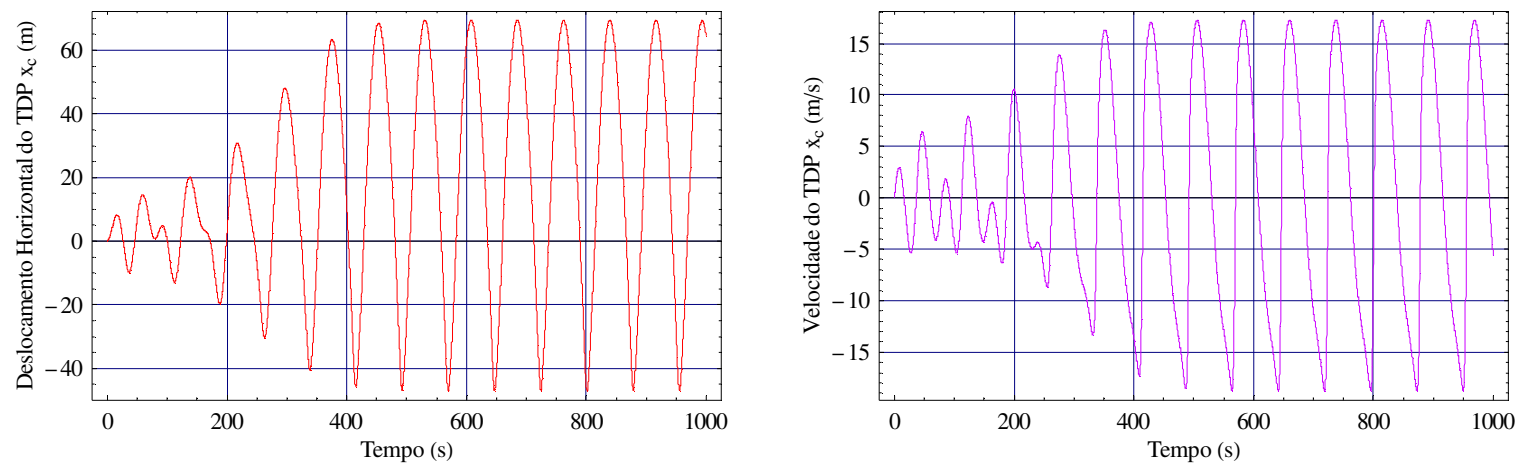

Figura 50: Deslocamento horizontal do $T D P x_{c}$ e velocidade $v_{c}$ para $\phi=10^{4} \mathrm{~N} / \mathrm{m}^{3}, T_{0, T D P=20 k N}, \xi=10 \%$, $c_{0}=7.44366, T=10.2 s$ para $x_{c, 0}=0.1$ e $v_{c, 0}=0.03$
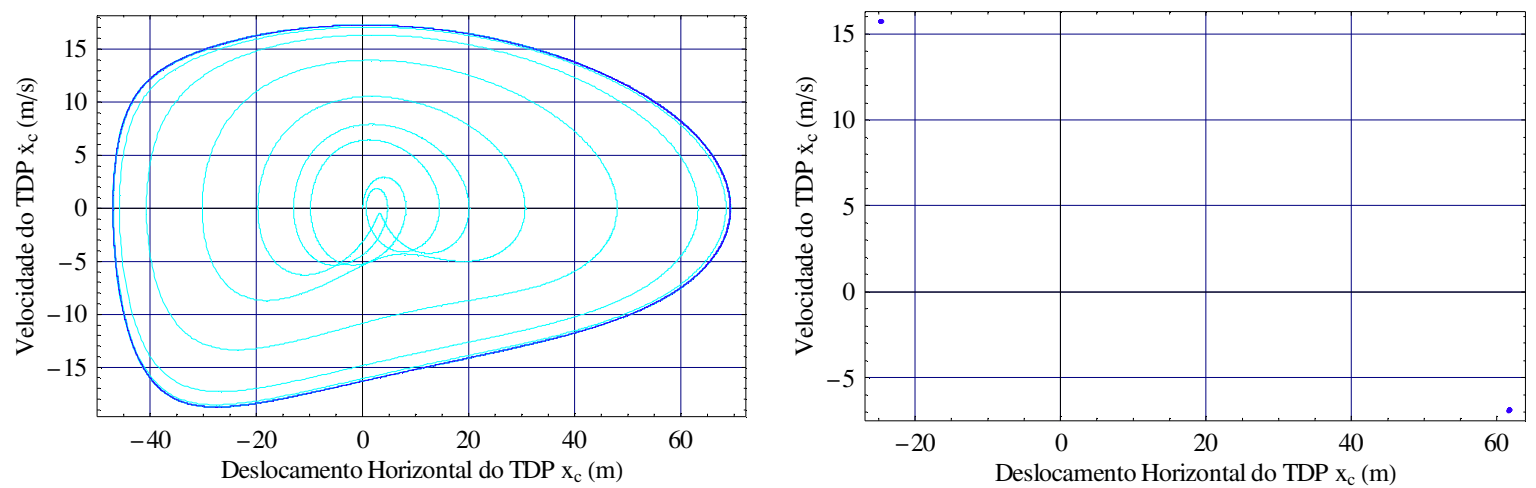

Figura 51: Mapa de fase e mapa de Poincaré para $\phi=10^{4} \mathrm{~N} / \mathrm{m}^{3}, \mathrm{~T}_{0, T D P}=20 \mathrm{kN}, \xi=10 \%, \mathrm{c}_{0}=7.44366, T=10.2 \mathrm{~s}$ para $x_{c, 0}=0.1$ e $v_{c, 0}=0.03$ 


\section{Equação do modelo de ordem reduzida para o caso P2:}

$$
\begin{aligned}
\ddot{U}+0.020349 \dot{U}+ & {[0.010352-0.005877 \cos (0.20349 t)] U } \\
& =0.035400 \cos (0.20349 t)+[0.011162+0.00094 \cos (0.20349 t)] U^{2}-0.033357 \dot{U}^{2} \\
& -0.619477 U \ddot{U}+[0.001977+0.000156 \cos (0.20349 t)] U^{3}+0.041294 U \dot{U}^{2} \\
& -0.143346 U^{2} \ddot{U}-0.000109 U^{4}+0.016297 U^{2} \dot{U}^{2}-0.014676 U^{3} \ddot{U}-0.000004 U^{5} \\
& +0.001415 U^{3} \dot{U}^{2}-0.00056 U^{4} \ddot{U}
\end{aligned}
$$
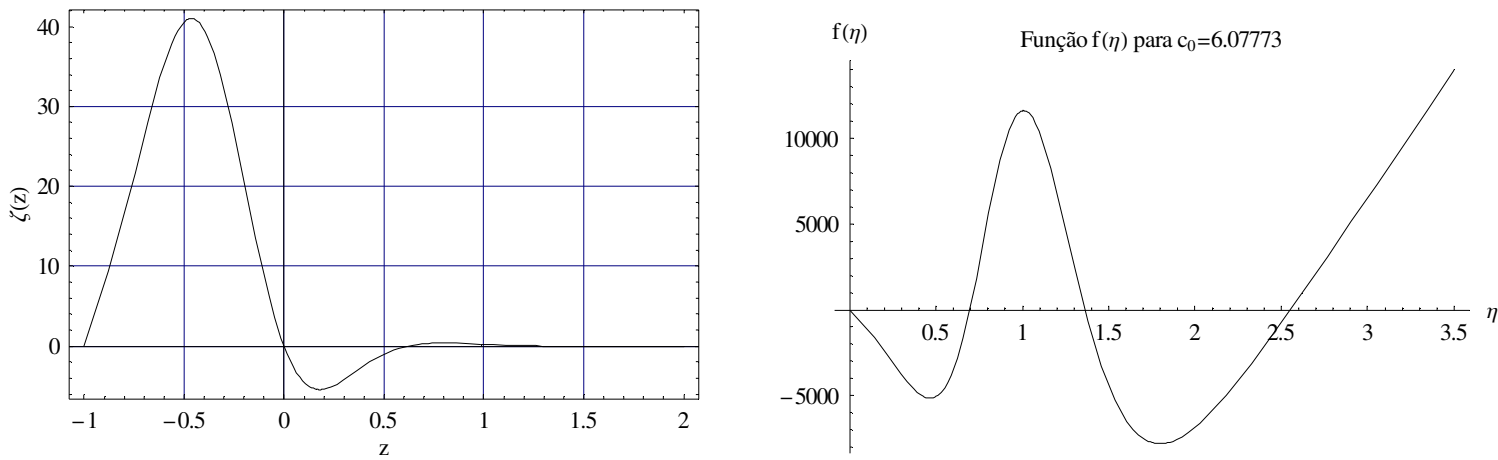

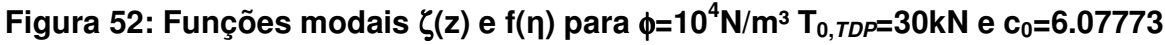
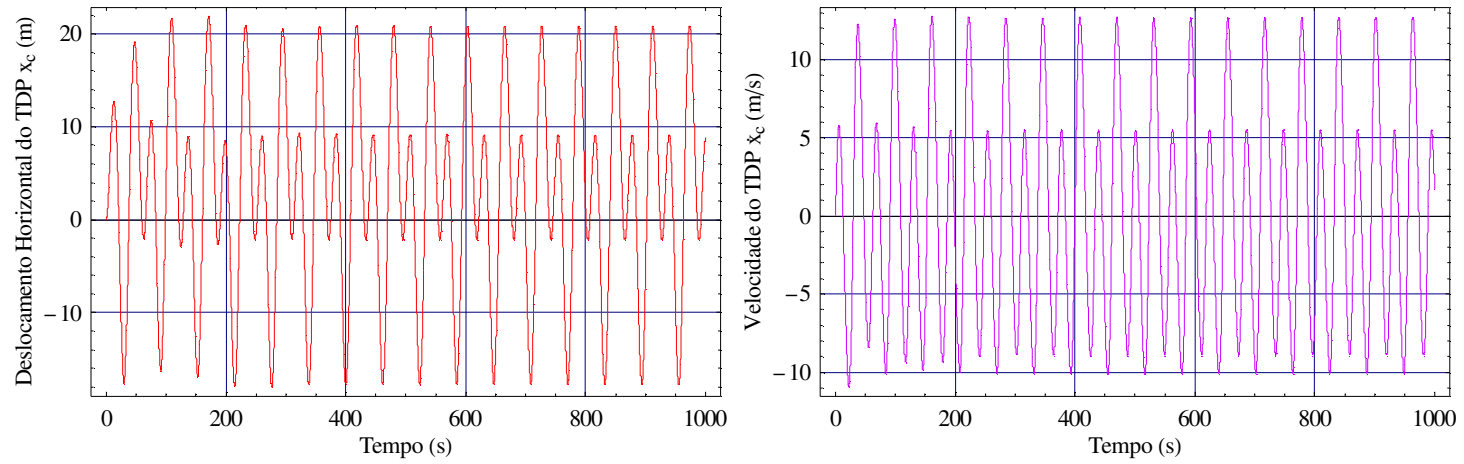

Figura 53: Deslocamento horizontal do $T D P x_{c}$ e velocidade $v_{c}$ para $\phi=10^{4} \mathrm{~N} / \mathrm{m}^{3}, T_{0, T D P}=30 \mathrm{kN}, \xi=10 \%$, $C_{0}=6.07773, T=8.1 s$ para $x_{c, 0}=0.1$ e $v_{c, 0}=0.04$
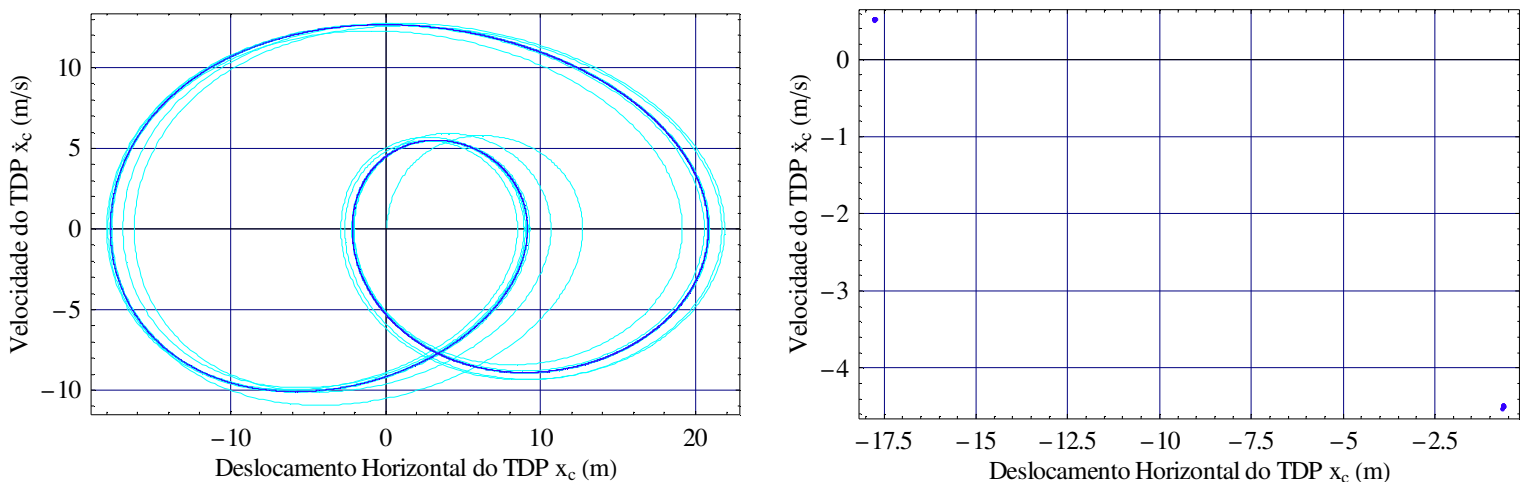

Figura 54: Mapa de fase e mapa de Poincaré para $\phi=10^{4} \mathrm{~N} / \mathrm{m}^{3}, \mathrm{~T}_{0, T D P}=30 \mathrm{kN}, \xi=10 \%, \mathrm{c}_{0}=6.07773, \mathrm{~T}=8.1 \mathrm{~s}$ para $x_{c, 0}=0.1$ e $v_{c, 0}=0.04$ 


\section{Equação do modelo de ordem reduzida para o caso P3:}

$$
\begin{aligned}
\ddot{U}+0.040724 \dot{U}+ & {[0.041463-0.001973 \cos (0.407249 t)] U } \\
& =0.043043 \cos (0.407249 t)+[0.032431+0.003911 \cos (0.407249 t)] U^{2} \\
& -0.011693 \dot{U}^{2}-0.719952 U \ddot{U}+[0.004453+0.000519 \cos (0.407249 t)] U^{3} \\
& +0.02385 U \dot{U}^{2}-0.193774 U^{2} \ddot{U}-0.000073 U^{4}+0.010328 U^{2} \dot{U}^{2}-0.023099 U^{3} \ddot{U} \\
& -0.000002 U^{5}+0.001021 U^{3} \dot{U}^{2}-0.001028 U^{4} \ddot{U}
\end{aligned}
$$
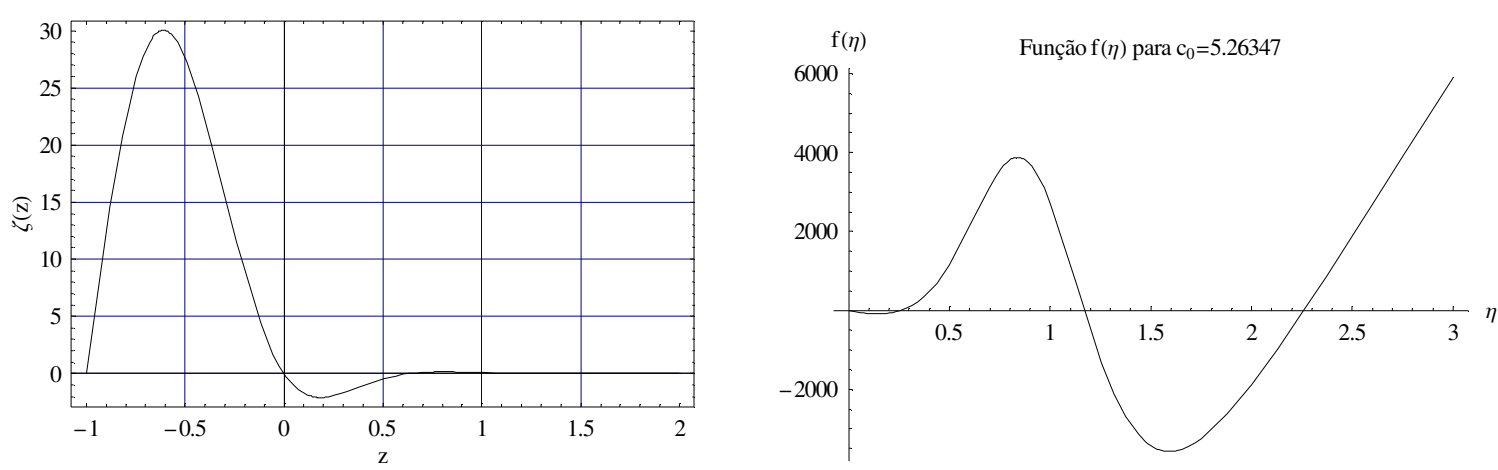

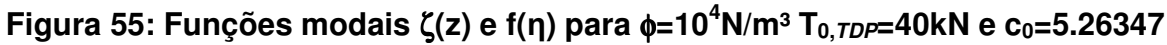
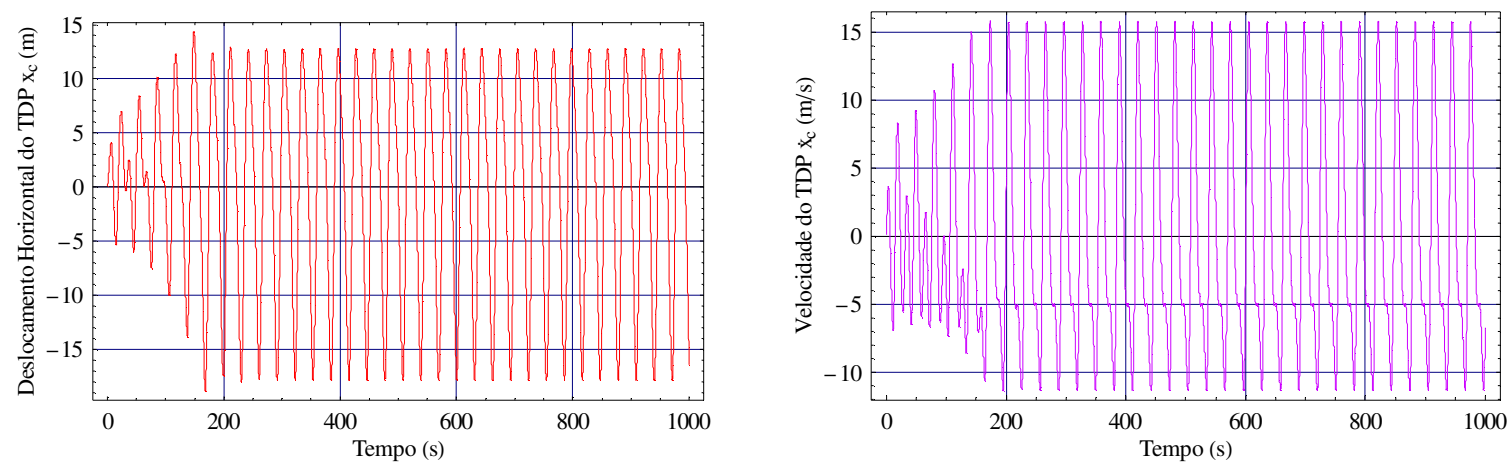

Figura 56: Deslocamento horizontal do $T D P x_{c}$ e velocidade $v_{c}$ para $\phi=10^{4} \mathrm{~N} / \mathrm{m}^{3}, T_{0, T D P}=40 \mathrm{kN}, \xi=10 \%$, $\mathrm{C}_{0}=5.26347, \mathrm{~T}=4.1 \mathrm{~s}$ para $\mathrm{x}_{\mathrm{c}, 0}=0.1$ e $\mathrm{v}_{\mathrm{c}, 0}=0.08$
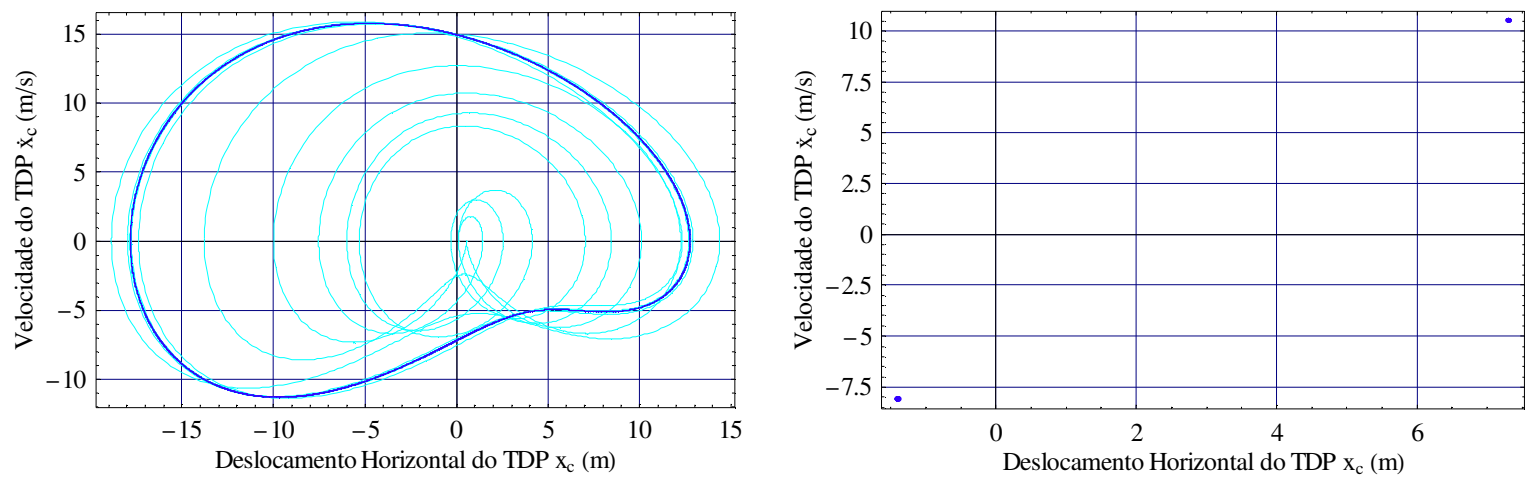

Figura 57: Mapa de fase e mapa de Poincaré para $\phi=10^{4} \mathrm{~N} / \mathrm{m}^{3}, \mathrm{~T}_{0, T D P=40 \mathrm{kN}}, \xi=10 \%, \mathrm{c}_{0}=5.26347, \mathrm{~T}=4.1 \mathrm{~s}$ para $x_{c, 0}=0.1$ e $v_{c, 0}=0.08$ 


\section{Equação do modelo de ordem reduzida para o caso P4:}

$$
\begin{aligned}
\ddot{U}+0.055057 \dot{U}+ & {[0.075784-0.00066 \cos (0.550578 t)] U } \\
& =0.069736 \cos (0.550578 t)+[0.072463+0.009158 \cos (0.550578 t)] U^{2} \\
& -0.011174 \dot{U}^{2}-0.805696 U \ddot{U}+[0.010856+0.001306 \cos (0.550578 t)] U^{3} \\
& +0.035613 U \dot{U}^{2}-0.242705 U^{2} \ddot{U}-0.000198 U^{4}+0.016642 U^{2} \dot{U}^{2}-0.032385 U^{3} \ddot{U} \\
& -0.000007 U^{5}+0.001821 U^{3} \dot{U}^{2}-0.001614 U^{4} \ddot{U}
\end{aligned}
$$
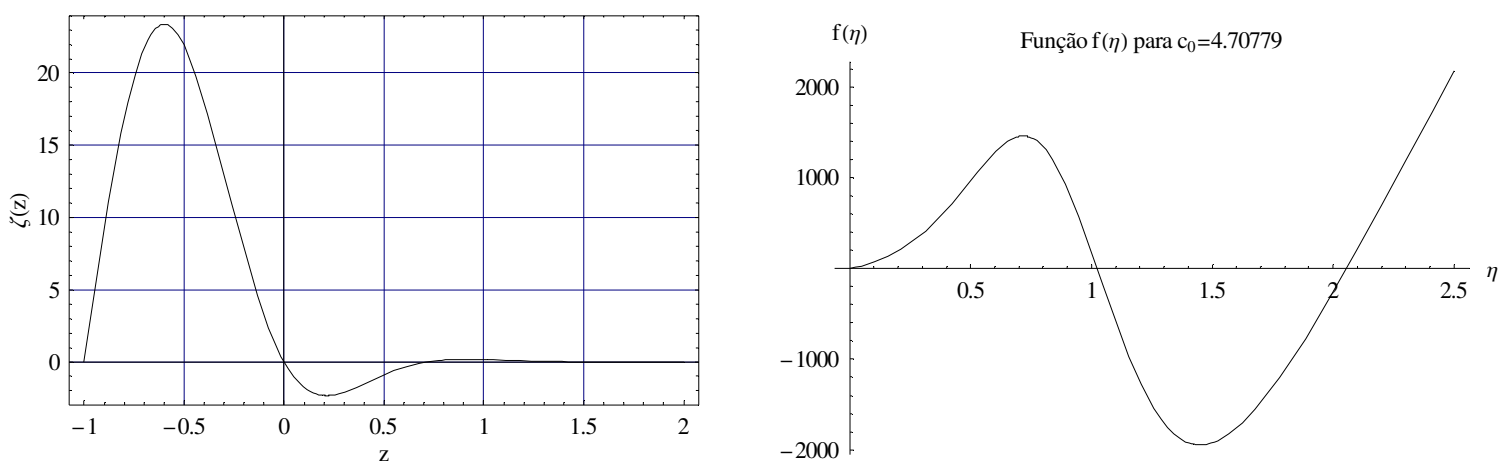

Figura 58: Funções modais $\zeta(z)$ e $f(\eta)$ para $\phi=10^{4} \mathrm{~N} / \mathrm{m}^{3} \mathrm{~T}_{0, T D P}=75 \mathrm{kN}$ e $c_{0}=4.70779$
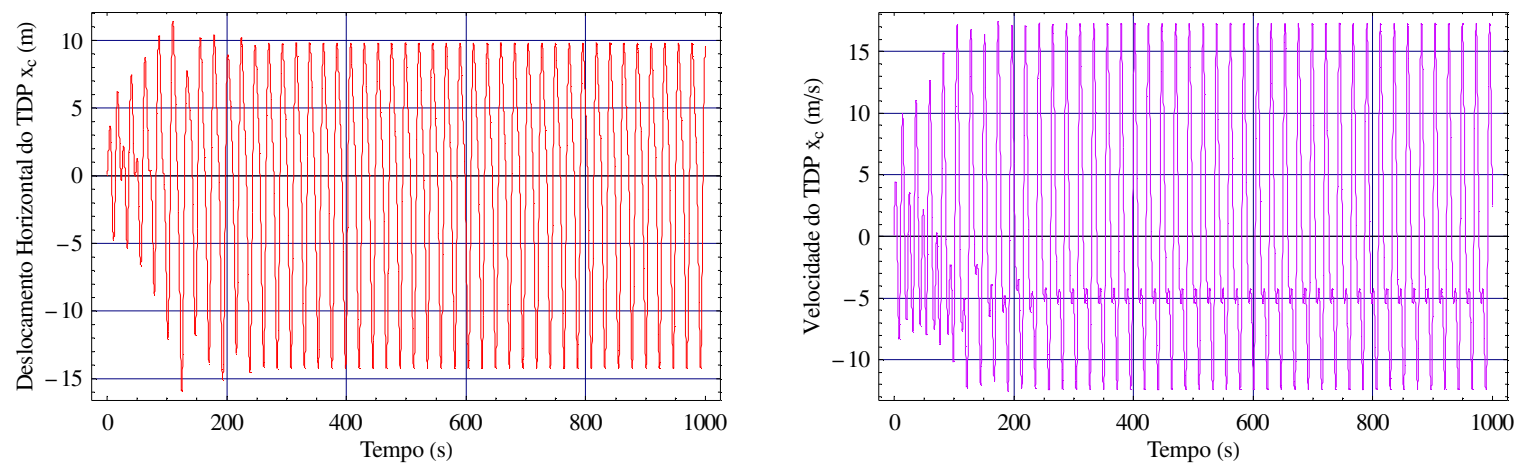

Figura 59: Deslocamento horizontal do $T D P x_{c}$ e velocidade $v_{c}$ para $\phi=10^{4} \mathrm{~N} / \mathrm{m}^{3}, T_{0, T D P=50 k N}, \xi=10 \%$, $c_{0}=4.70779, T=3.0 s$ para $x_{c, 0}=0.1$ e $v_{c, 0}=0.1$
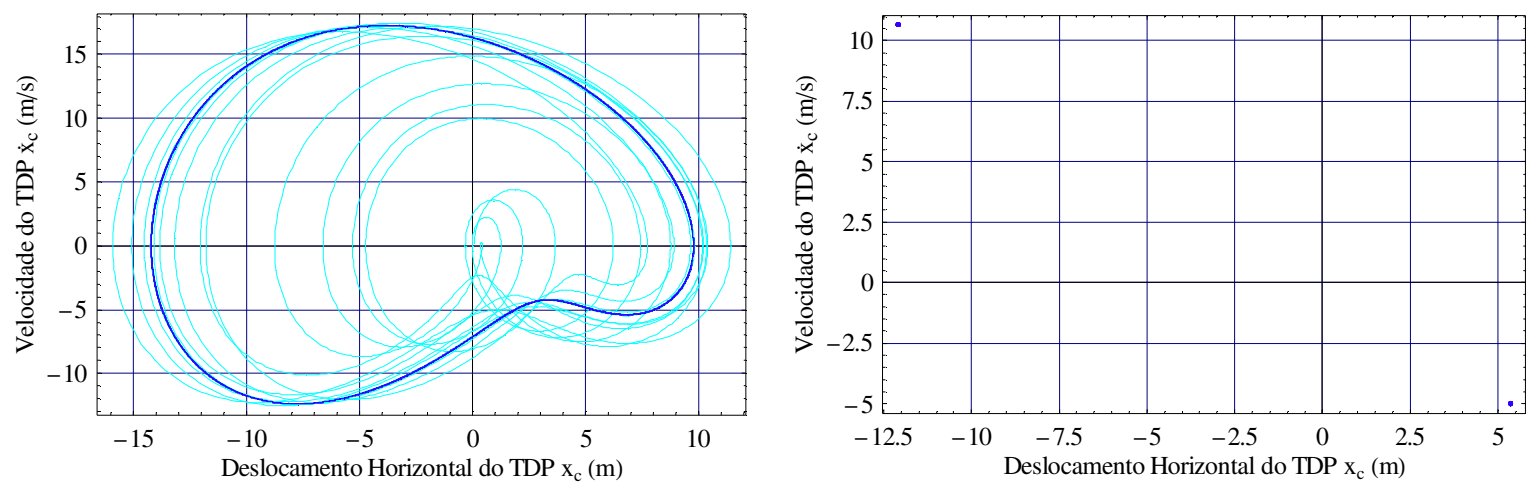

Figura 60: Mapa de fase e mapa de Poincaré para $\phi=10^{4} \mathrm{~N} / \mathrm{m}^{3}, \mathrm{~T}_{0, T D P=50 \mathrm{kN}}, \xi=10 \%, \mathrm{C}_{0}=4.70779, \mathrm{~T}=3.0 \mathrm{~s}$ para $x_{c, 0}=0.1$ e $v_{c, 0}=0.1$ 


\section{Equação do modelo de ordem reduzida para o caso P5:}

$\ddot{U}+0.01176 \dot{U}+[0.003457-0.000609 \cos (0.117608 t)] U$

$$
\begin{aligned}
& =0.007523 \cos (0.117608 t)+[0.00129+0.000127 \cos (0.117608 t)] U^{2} \\
& -0.022250 \dot{U}^{2}-0.401607 U \ddot{U}+[0.000111+0.000011 \cos (0.117608 t)] U^{3} \\
& +0.011337 U \dot{U}^{2}-0.060234 U^{2} \ddot{U}-0.000001 U^{4}+0.003184 U^{2} \dot{U}^{2}-0.003996 U^{3} \ddot{U} \\
& -0.000001 U^{5}+0.000183 U^{3} \dot{U}^{2}-0.000098 U^{4} \ddot{U}
\end{aligned}
$$
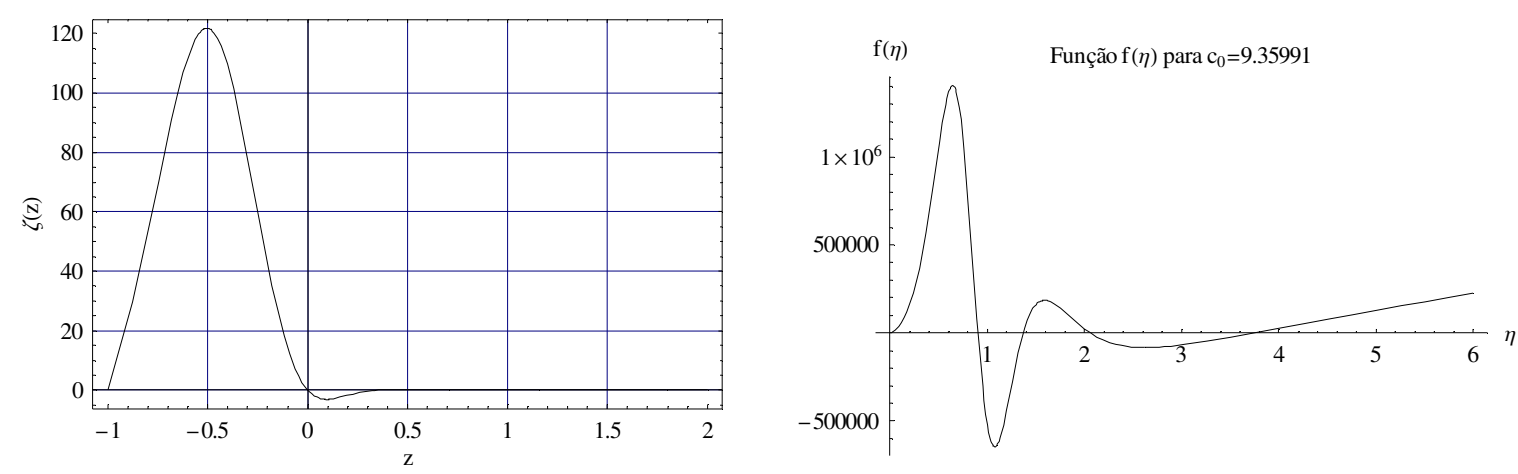

Figura 61: Funções modais $\zeta(z)$ e $f(\eta)$ para $\phi=10^{5} \mathrm{~N} / \mathrm{m}^{3} \mathrm{~T}_{0, T D P=40 \mathrm{kN} \text { e } c_{0}=9.35991}$
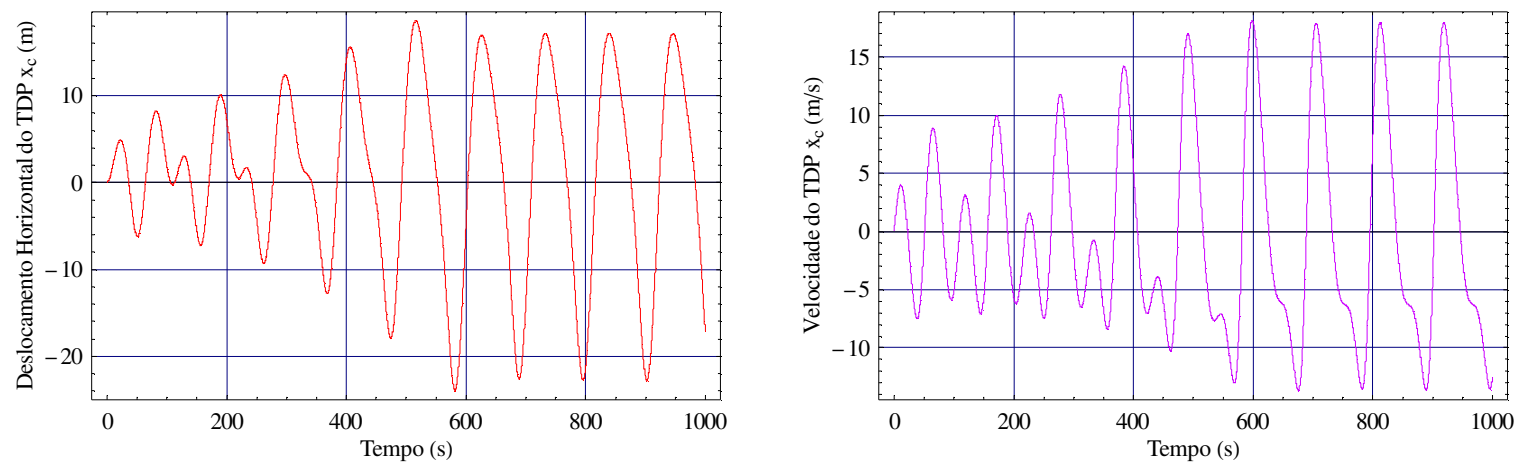

Figura 62: Deslocamento horizontal do $T D P x_{c}$ e velocidade $v_{c}$ para $\phi=10^{5} \mathrm{~N} / \mathrm{m}^{3}, \mathrm{~T}_{0, T D P=40 \mathrm{kN}}, \xi=10 \%$, $C_{0}=9.35991, T=4.5 s$ para $x_{c, 0}=0.1$ e $v_{c, 0}=0.07$
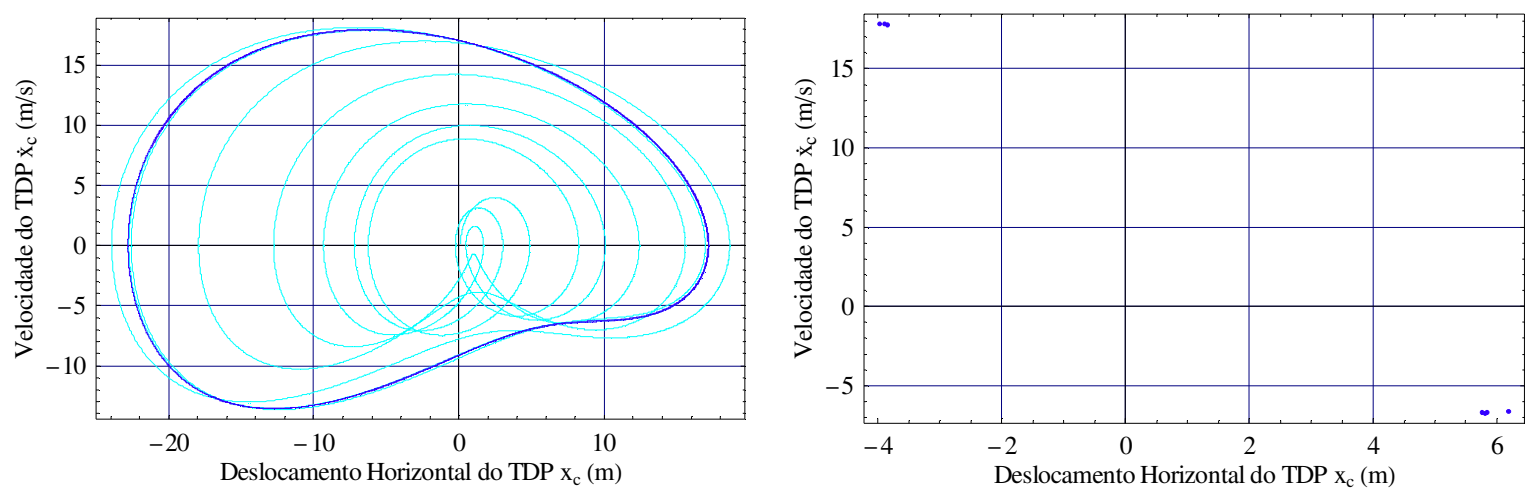

Figura 63: Mapa de fase e mapa de Poincaré para $\phi=10^{5} \mathrm{~N} / \mathrm{m}^{3}, \mathrm{~T}_{0, T D P}=40 \mathrm{kN}, \xi=10 \%, \mathrm{c}_{0}=9.35991, \mathrm{~T}=4.5 \mathrm{~s}$ para $x_{c, 0}=0.1$ e $v_{c, 0}=0.07$ 


\section{Equação do modelo de ordem reduzida para o caso P6:}

$$
\begin{aligned}
\ddot{U}+0.016739 \dot{U}+ & {[0.007004-0.000993 \cos (0.167391 t)] U } \\
& =0.014103 \cos (0.167391 t)+[0.003943+0.000366 \cos (0.167391 t)] U^{2} \\
& -0.027897 \dot{U}^{2}-0.448541 U \ddot{U}+[0.000423+0.000033 \cos (0.167391 t)] U^{3} \\
& +0.015356 U \dot{U}^{2}-0.075125 U^{2} \ddot{U}-0.000012 U^{4}+0.004862 U^{2} \dot{U}^{2}-0.005565 U^{3} \ddot{U} \\
& -0.000001 U^{5}+0.000314 U^{3} \dot{U}^{2}-0.000153 U^{4} \ddot{U}
\end{aligned}
$$
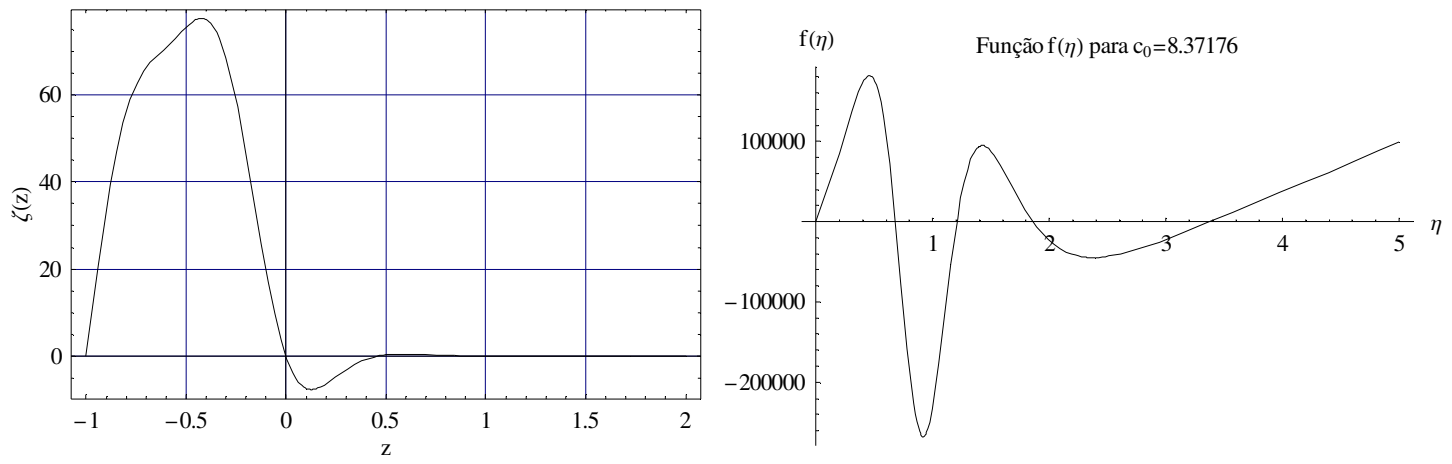

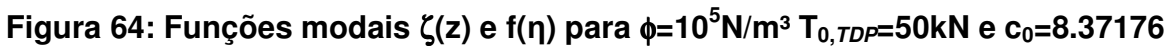
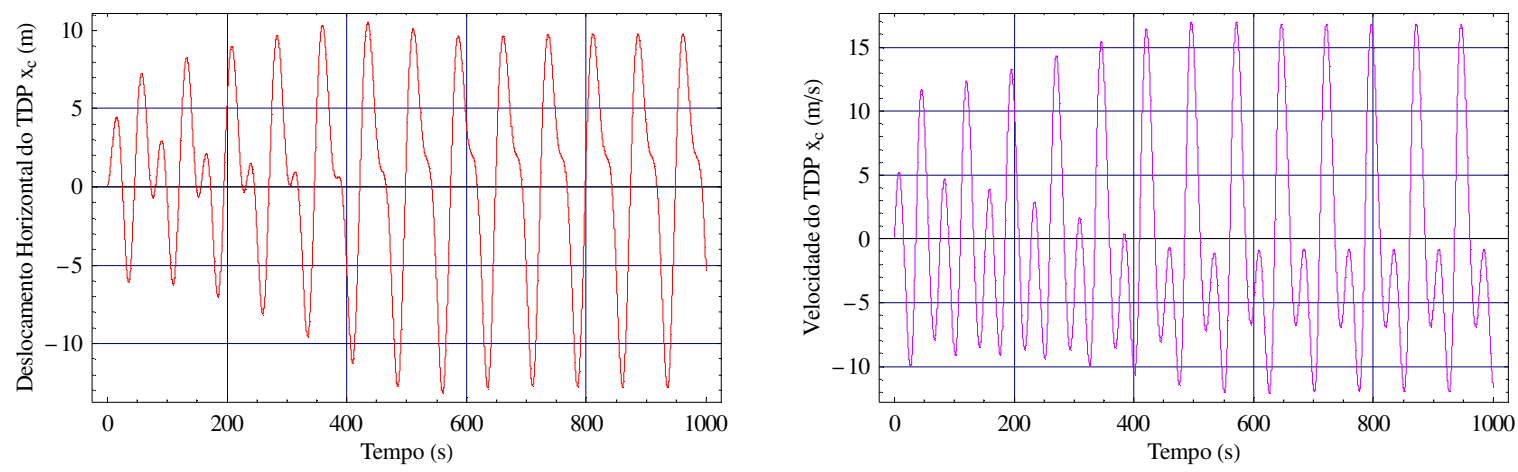

Figura 65: Deslocamento horizontal do $T D P x_{c}$ e velocidade $v_{c}$ para $\phi=10^{5} \mathrm{~N} / \mathrm{m}^{3}, T_{0, T D P=50 k N}, \xi=10 \%$, $C_{0}=8.37176, T=3.1 s$ para $x_{c, 0}=0.1$ e $v_{c, 0}=0.1$
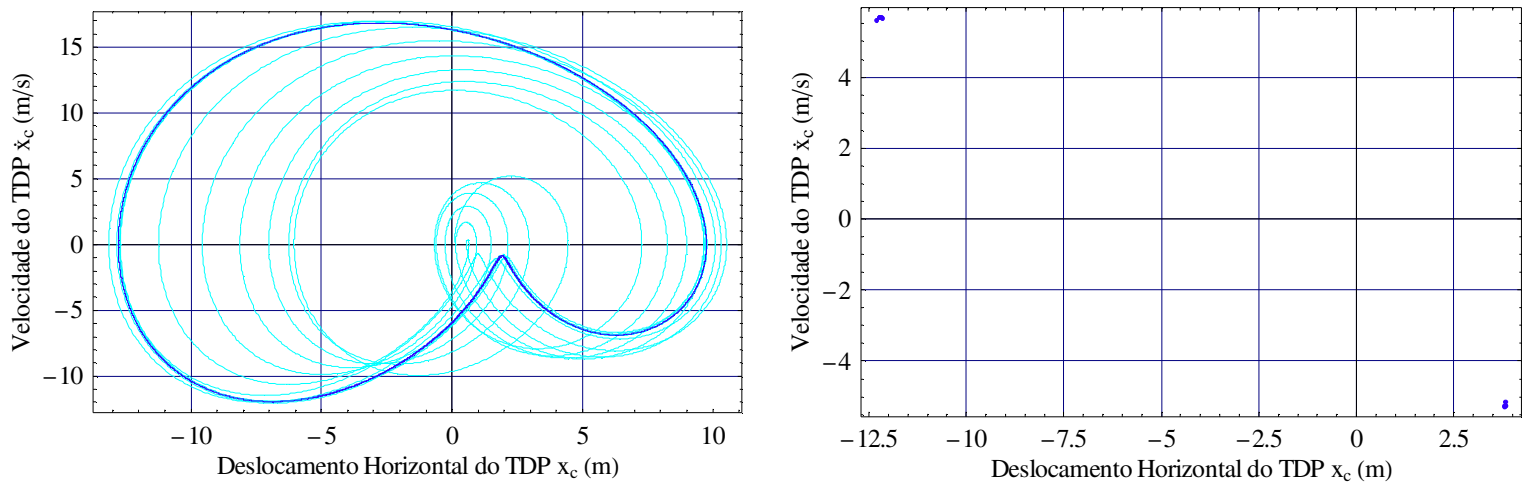

Figura 66: Mapa de fase e mapa de Poincaré para $\phi=10^{5} \mathrm{~N} / \mathrm{m}^{3}, \mathrm{~T}_{0, T D P}=50 \mathrm{kN}, \xi=10 \%, \mathrm{C}_{0}=8.37176, \mathrm{~T}=3.1 \mathrm{~s}$ para $x_{c, 0}=0.1$ e $v_{c, 0}=0.1$ 


\section{Equação do modelo de ordem reduzida para o caso P7:}

$$
\begin{aligned}
\ddot{U}+0.048848 \dot{U}+ & {[0.006628-0.002163 \cos (0.162829 t)] U } \\
& =0.013551 \cos (0.162829 t)+[0.002259+0.000152 \cos (0.162829 t)] U^{2} \\
& -0.027649 \dot{U}^{2}-0.50514 U \ddot{U}+[0.000308+0.000026 \cos (0.162829 t)] U^{3} \\
& +0.020408 U \dot{U}^{2}-0.095297 U^{2} \ddot{U}-0.00001 U^{4}+0.00698 U^{2} \dot{U}^{2}-0.007953 U^{3} \ddot{U} \\
& -0.000001 U^{5}+0.000502 U^{3} \dot{U}^{2}-0.000247 U^{4} \ddot{U}
\end{aligned}
$$
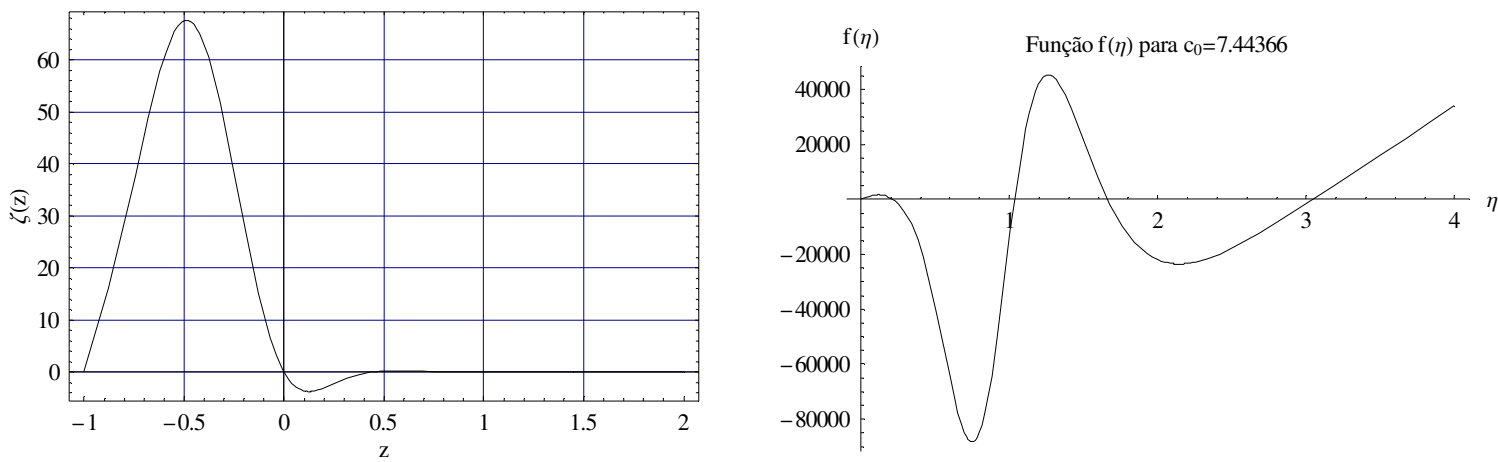

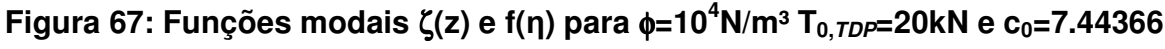
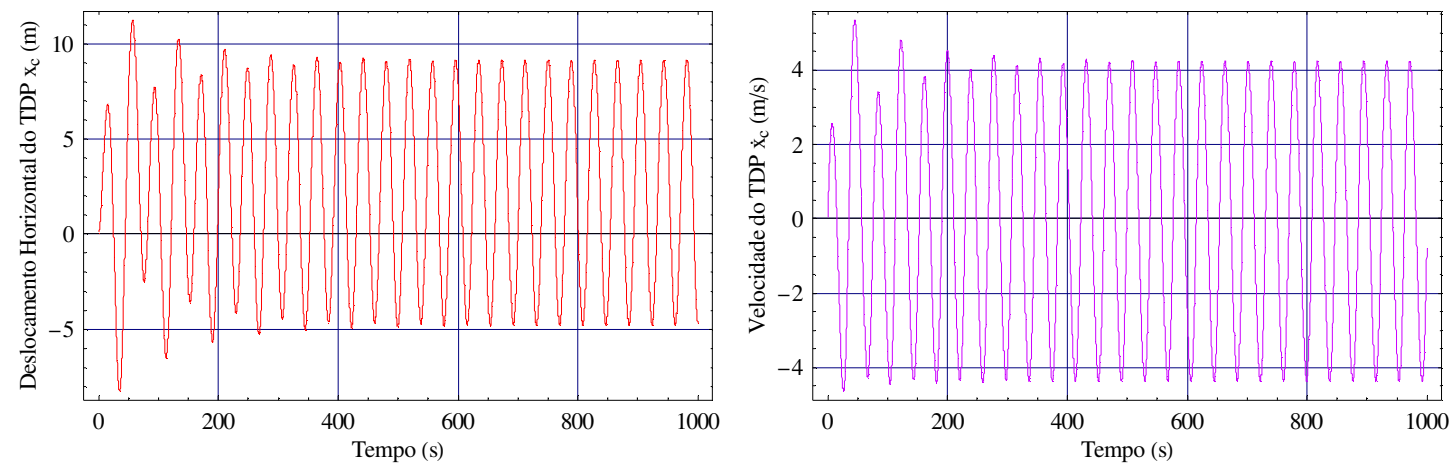

Figura 68: Deslocamento horizontal do $T D P x_{c}$ e velocidade $v_{c}$ para $\phi=10^{4} \mathrm{~N} / \mathrm{m}^{3}, T_{0, T D P=20 k N}, \xi=20 \%$, $c_{0}=7.44366, T=10.2 s$ para $x_{c, 0}=0.1$ e $v_{c, 0}=0.03$
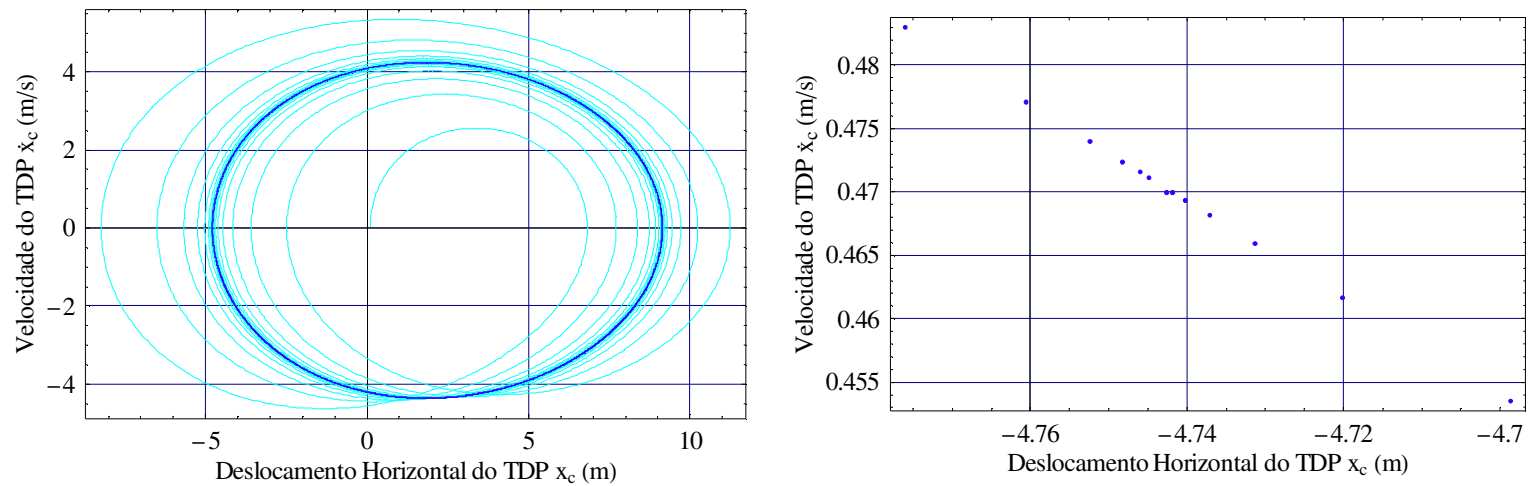

Figura 69: Mapa de fase e mapa de Poincaré para $\phi=10^{4} \mathrm{~N} / \mathrm{m}^{3}, \mathrm{~T}_{0, T D P}=20 \mathrm{kN}, \xi=20 \%, \mathrm{c}_{0}=7.44366, T=10.2 \mathrm{~s}$ para $x_{c, 0}=0.1$ e $v_{c, 0}=0.03$ 


\section{Equação do modelo de ordem reduzida para o caso P8:}

$$
\begin{aligned}
\ddot{U}+0.061047 \dot{U}+ & {[0.010352-0.005877 \cos (0.20349 t)] U } \\
& =0.035400 \cos (0.20349 t)+[0.011162+0.00094 \cos (0.20349 t)] U^{2}-0.033357 \dot{U}^{2} \\
& -0.619477 U \ddot{U}+[0.001977+0.000156 \cos (0.20349 t)] U^{3}+0.041294 U \dot{U}^{2} \\
& -0.143346 U^{2} \ddot{U}-0.000109 U^{4}+0.016297 U^{2} \dot{U}^{2}-0.014676 U^{3} \ddot{U}-0.000004 U^{5} \\
& +0.001415 U^{3} \dot{U}^{2}-0.00056 U^{4} \ddot{U}
\end{aligned}
$$
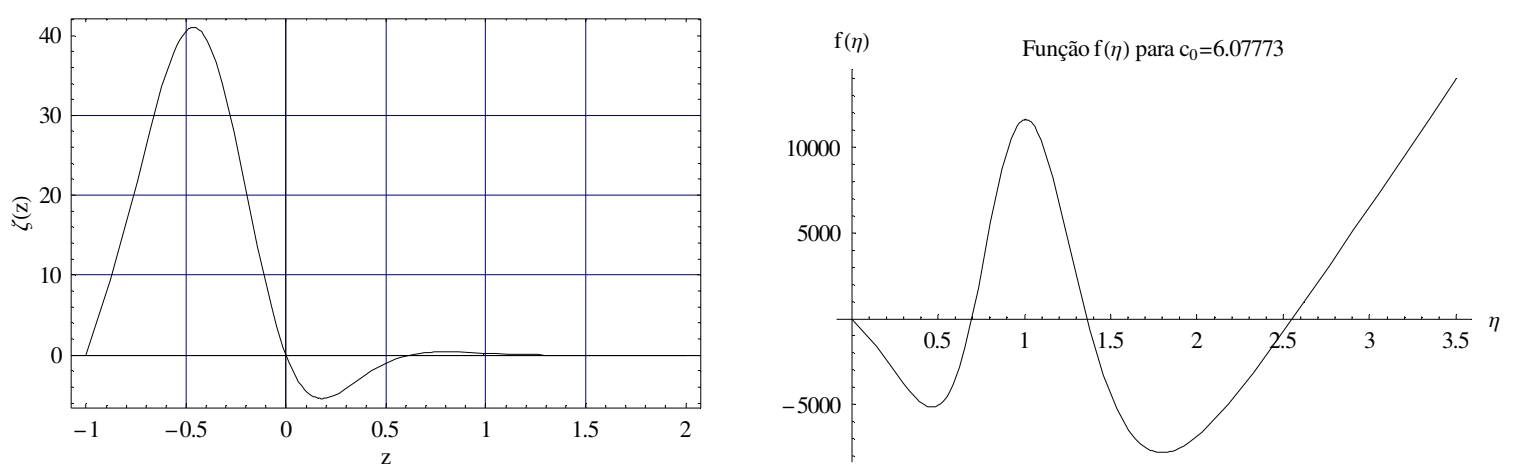

Figura 70: Funções modais $\zeta(z)$ e $f(\eta)$ para $\phi=10^{4} \mathrm{~N} / \mathrm{m}^{3} \mathrm{~T}_{0, T D P}=30 \mathrm{kN}$ e $\mathrm{c}_{0}=6.07773$
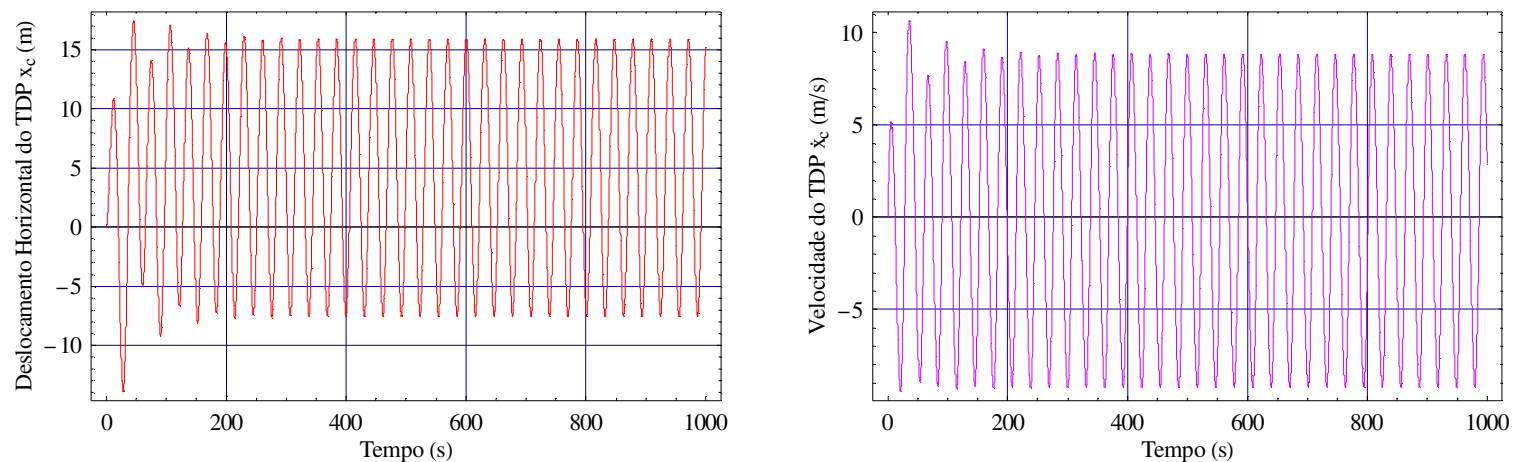

Figura 71: Deslocamento horizontal do TDP $x_{c}$ e velocidade $v_{c}$ para $\phi=10^{4} \mathrm{~N} / \mathrm{m}^{3}, T_{0, T D P=30 k N}, \xi=20 \%$, $C_{0}=6.07773, T=8.1 s$ para $x_{c, 0}=0.1$ e $v_{c, 0}=0.04$
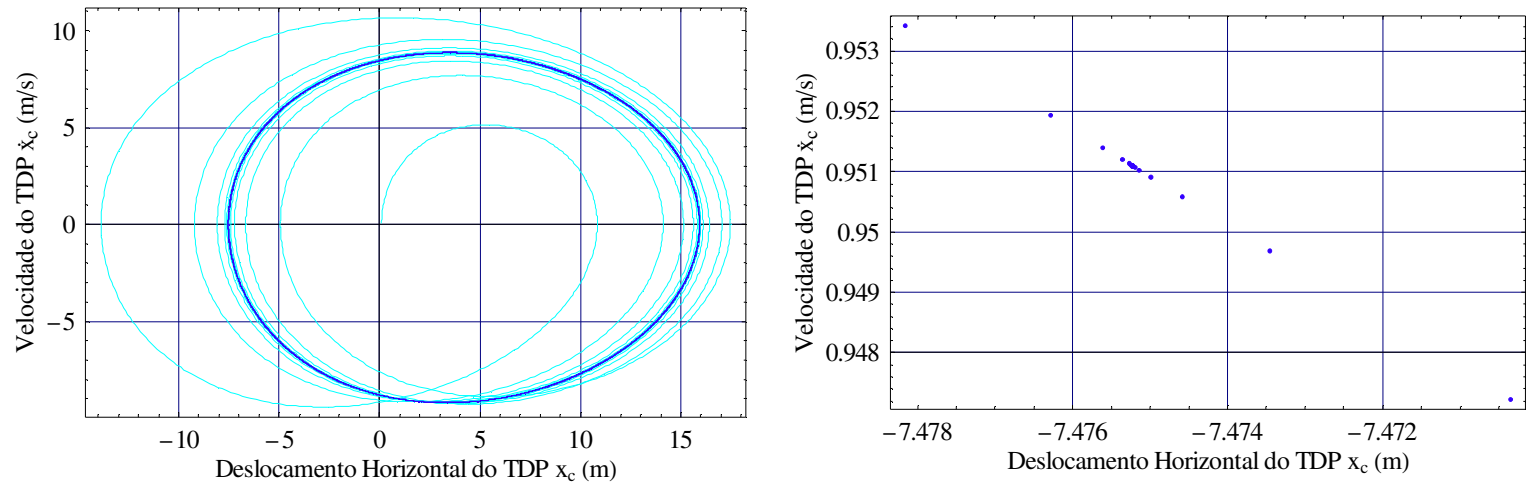

Figura 72: Mapa de fase e mapa de Poincaré para $\phi=10^{4} \mathrm{~N} / \mathrm{m}^{3}, \mathrm{~T}_{0, T D P}=30 \mathrm{kN}, \xi=20 \%, \mathrm{c}_{0}=6.07773, \mathrm{~T}=8.1 \mathrm{~s}$ para $x_{c, 0}=0.1$ e $v_{c, 0}=0.04$ 


\section{Equação do modelo de ordem reduzida para o caso P9:}

$$
\begin{aligned}
\ddot{U}+0.122174 \dot{U}+ & {[0.041463-0.001973 \cos (0.407249 t)] U } \\
& =0.043043 \cos (0.407249 t)+[0.032431+0.003911 \cos (0.407249 t)] U^{2} \\
& -0.011693 \dot{U}^{2}-0.719952 U \ddot{U}+[0.004453+0.000519 \cos (0.407249 t)] U^{3} \\
& +0.02385 U \dot{U}^{2}-0.193774 U^{2} \ddot{U}-0.000073 U^{4}+0.010328 U^{2} \dot{U}^{2}-0.023099 U^{3} \ddot{U} \\
& -0.000002 U^{5}+0.001021 U^{3} \dot{U}^{2}-0.001028 U^{4} \ddot{U}
\end{aligned}
$$
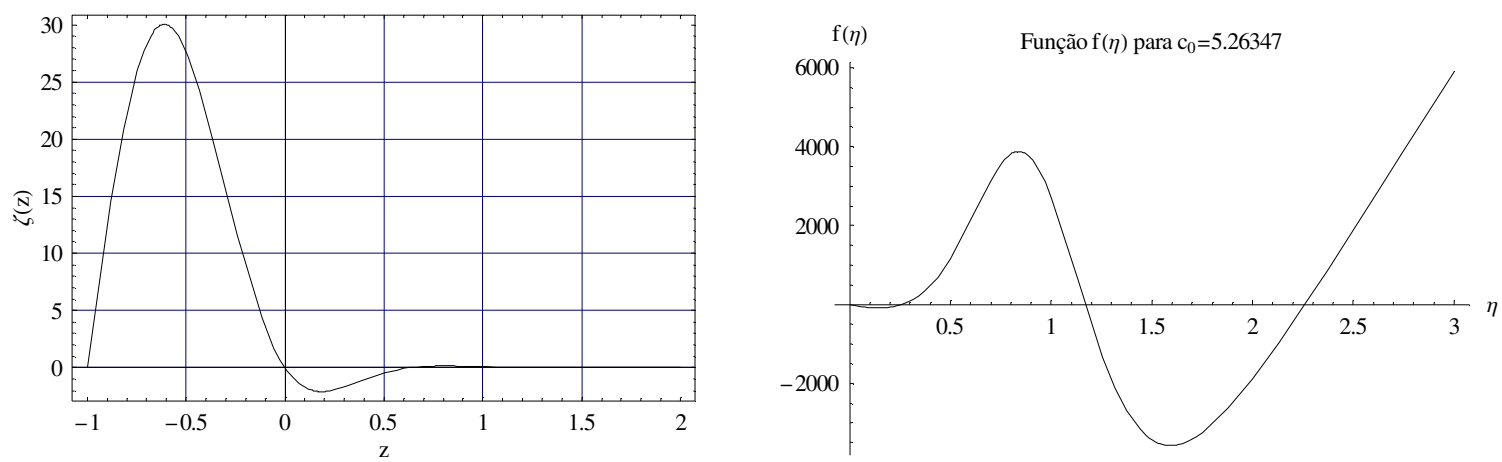

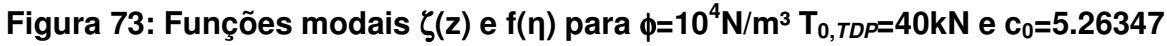
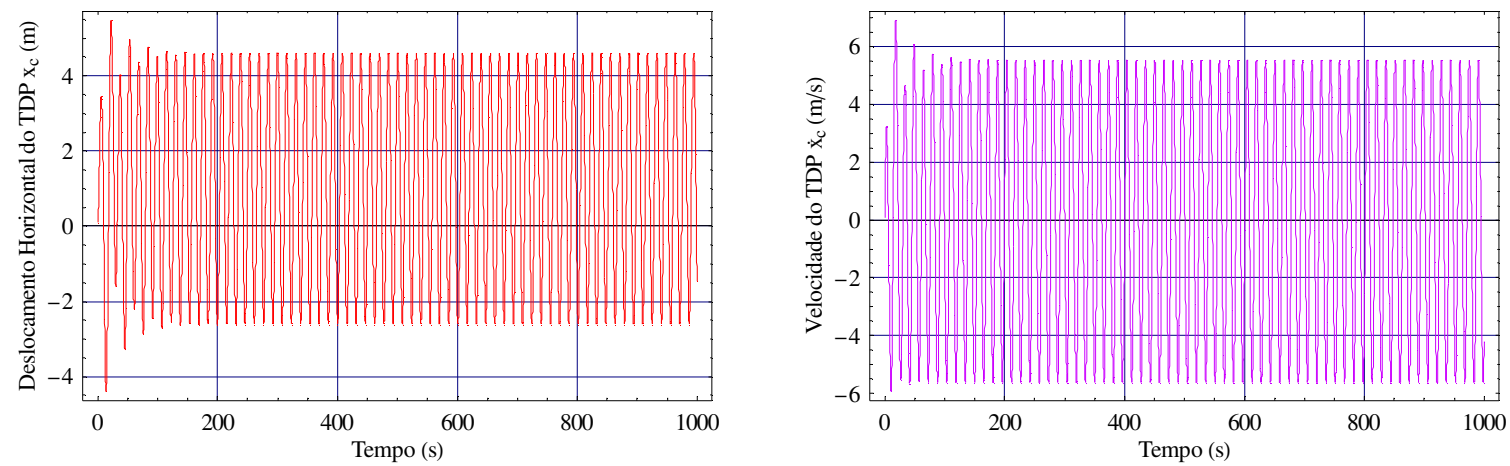

Figura 74: Deslocamento horizontal do $T D P \mathrm{x}_{\mathrm{c}}$ e velocidade $\mathrm{v}_{\mathrm{c}}$ para $\phi=10^{4} \mathrm{~N} / \mathrm{m}^{3}, \mathrm{~T}_{0, T D P=40 \mathrm{kN}}, \xi=20 \%$, $\mathrm{C}_{0}=5.26347, \mathrm{~T}=4.1 \mathrm{~s}$ para $\mathrm{x}_{\mathrm{c}, 0}=0.1$ e $\mathrm{v}_{\mathrm{c}, 0}=0.08$
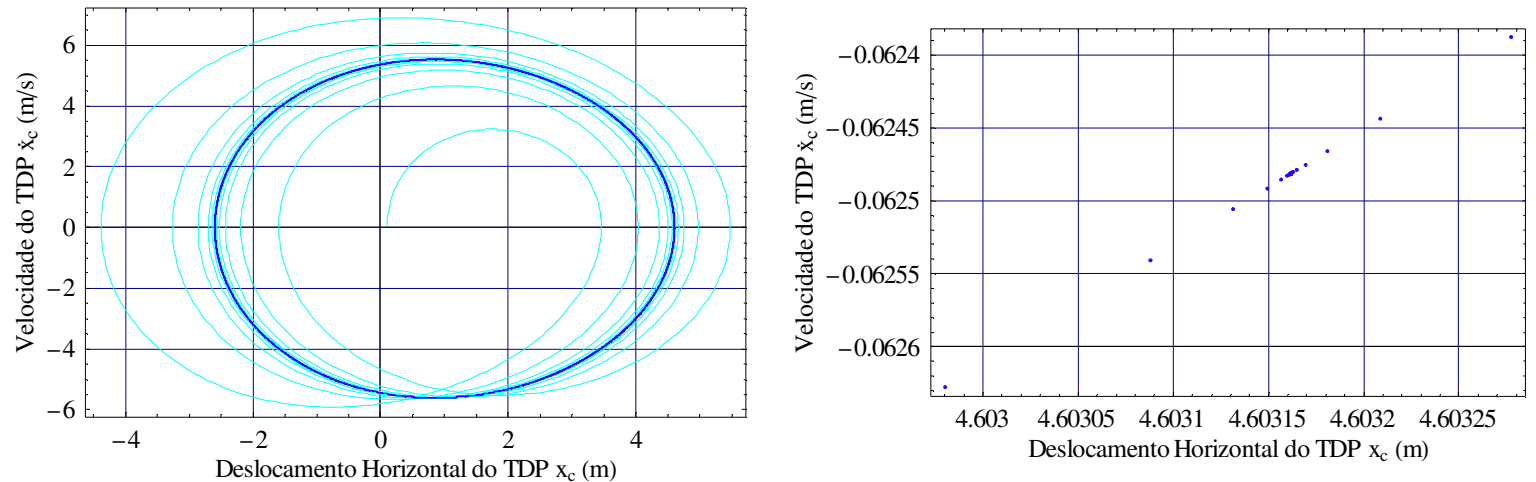

Figura 75: Mapa de fase e mapa de Poincaré para $\phi=10^{4} \mathrm{~N} / \mathrm{m}^{3}, \mathrm{~T}_{0, T D P=40 \mathrm{kN}}, \xi=20 \%, \mathrm{c}_{0}=5.26347$, T=4.1s para $x_{c, 0}=0.1$ e $v_{c, 0}=0.08$ 


\section{Equação do modelo de ordem reduzida para o caso P10:}

$$
\begin{aligned}
\ddot{U}+0.165173 \dot{U}+ & {[0.075784-0.00066 \cos (0.550578 t)] U } \\
& =0.069736 \cos (0.550578 t)+[0.072463+0.009158 \cos (0.550578 t)] U^{2} \\
& -0.011174 \dot{U}^{2}-0.805696 U \ddot{U}+[0.010856+0.001306 \cos (0.550578 t)] U^{3} \\
& +0.035613 U \dot{U}^{2}-0.242705 U^{2} \ddot{U}-0.000198 U^{4}+0.016642 U^{2} \dot{U}^{2}-0.032385 U^{3} \ddot{U} \\
& -0.000007 U^{5}+0.001821 U^{3} \dot{U}^{2}-0.001614 U^{4} \ddot{U}
\end{aligned}
$$
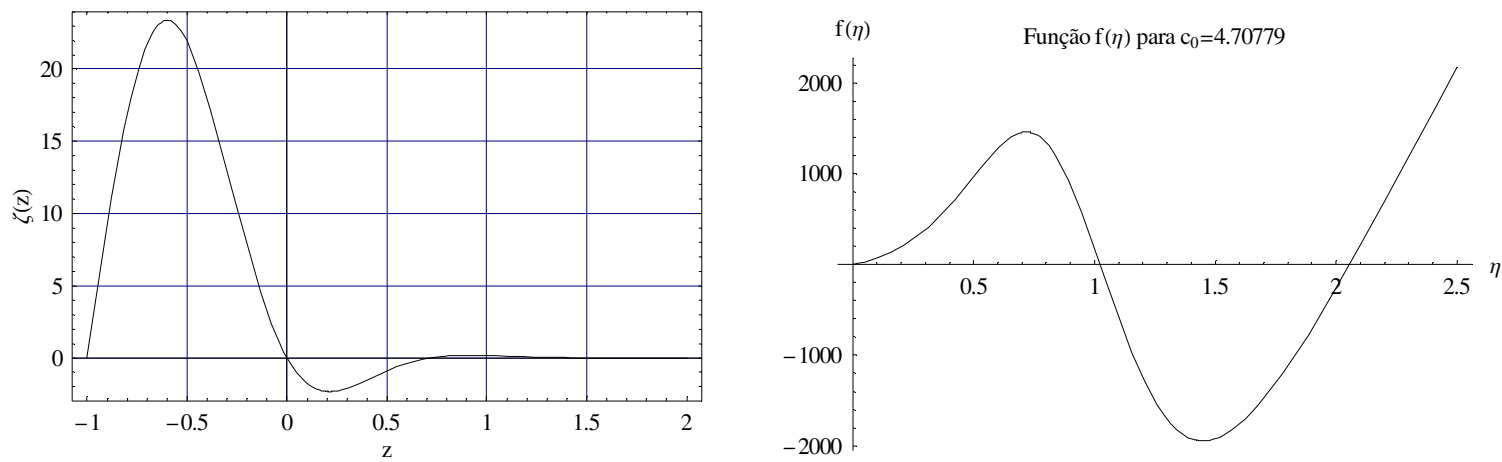

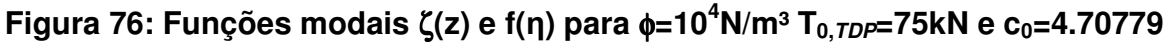
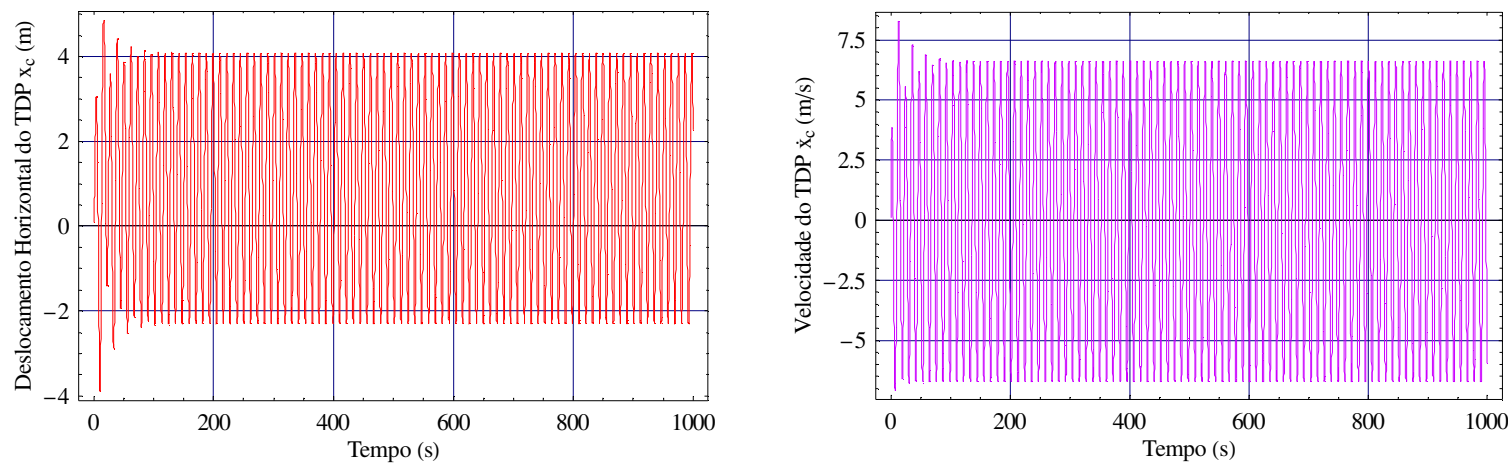

Figura 77: Deslocamento horizontal do $T D P \mathrm{x}_{\mathrm{c}}$ e velocidade $\mathrm{v}_{\mathrm{c}}$ para $\phi=10^{4} \mathrm{~N} / \mathrm{m}^{3}, \mathrm{~T}_{0, T D P=50 \mathrm{kN}}, \xi=20 \%$, $c_{0}=4.70779, T=3.0 s$ para $x_{c, 0}=0.1$ e $v_{c, 0}=0.1$
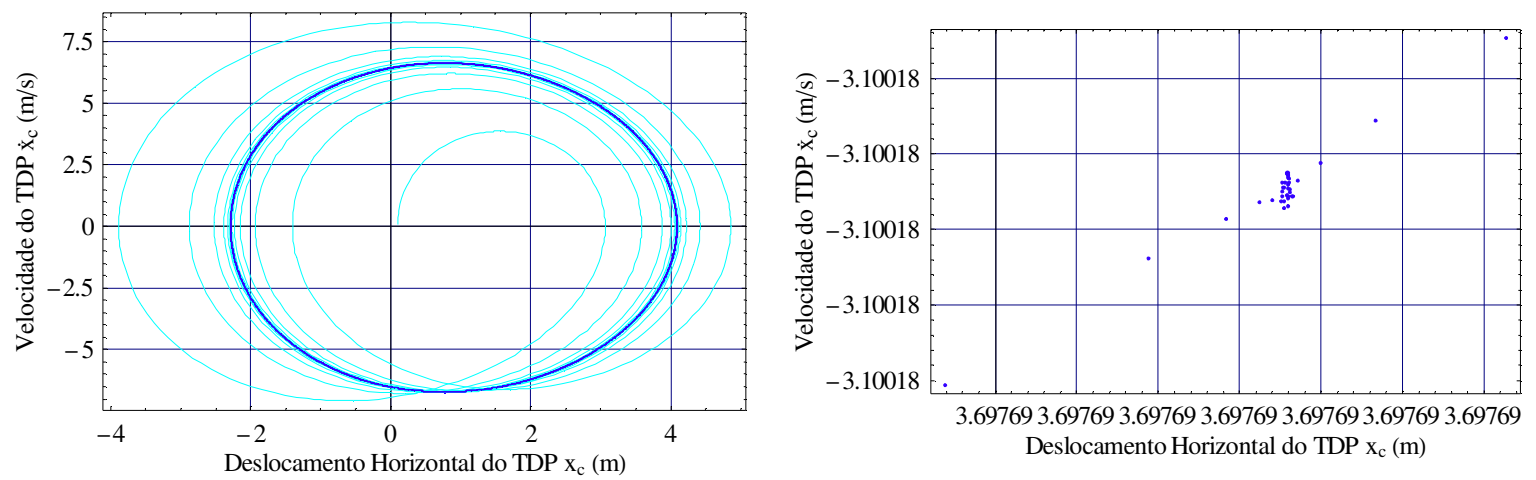

Figura 78: Mapa de fase e mapa de Poincaré para $\phi=10^{4} \mathrm{~N} / \mathrm{m}^{3}, \mathrm{~T}_{0, T D P=50 \mathrm{kN}}, \xi=20 \%, \mathrm{c}_{0}=4.70779$, T=3.0s para $x_{c, 0}=0.1$ e $v_{c, 0}=0.1$ 


\section{Equação do modelo de ordem reduzida para o caso P11:}

$$
\begin{aligned}
\ddot{U}+0.035282 \dot{U}+ & {[0.003457-0.000609 \cos (0.117608 t)] U } \\
& =0.007523 \cos (0.117608 t)+[0.00129+0.000127 \cos (0.117608 t)] U^{2} \\
& -0.022250 \dot{U}^{2}-0.401607 U \ddot{U}+[0.000111+0.000011 \cos (0.117608 t)] U^{3} \\
& +0.011337 U \dot{U}^{2}-0.060234 U^{2} \ddot{U}-0.000001 U^{4}+0.003184 U^{2} \dot{U}^{2}-0.003996 U^{3} \ddot{U} \\
& -0.000001 U^{5}+0.000183 U^{3} \dot{U}^{2}-0.000098 U^{4} \ddot{U}
\end{aligned}
$$
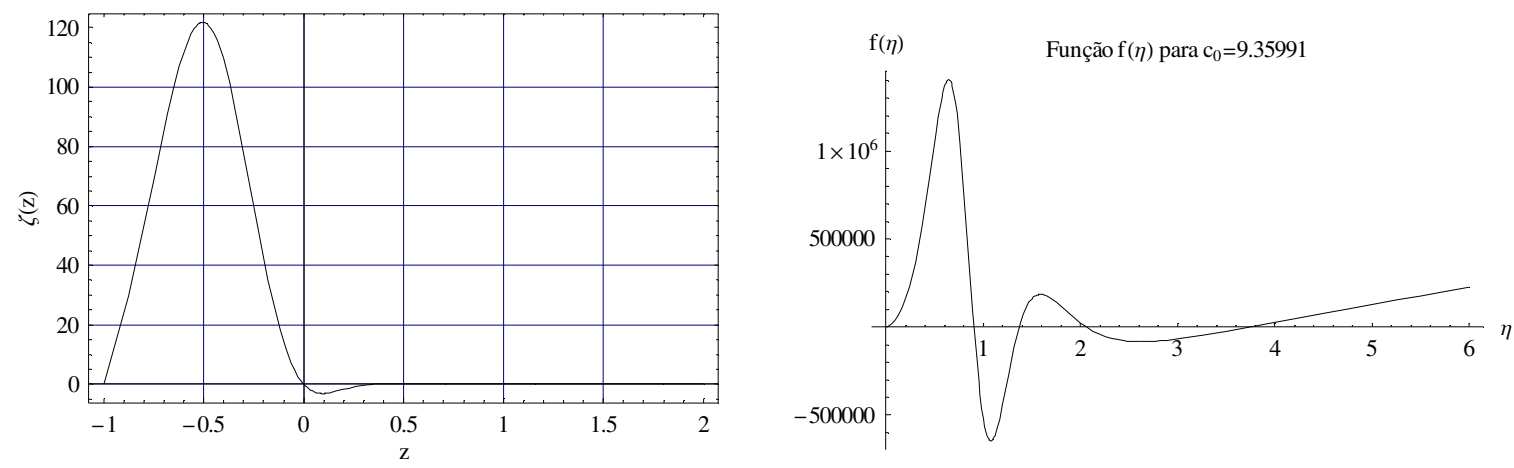

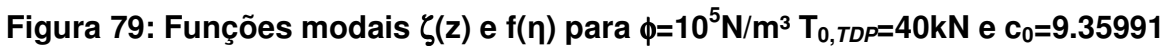
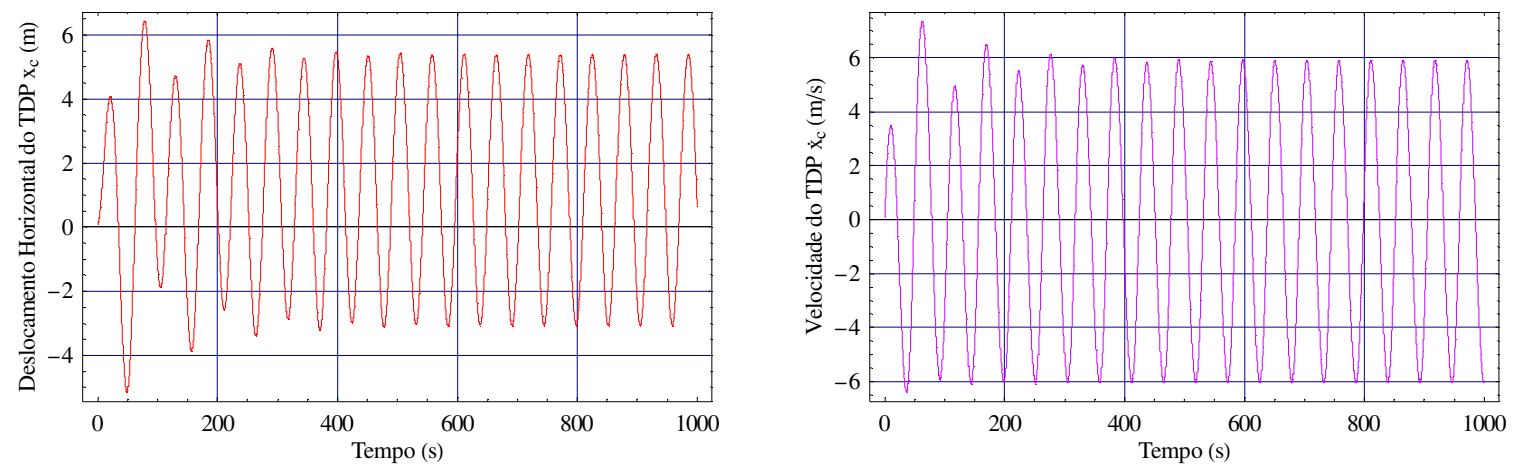

Figura 80: Deslocamento horizontal do $T D P \mathrm{x}_{\mathrm{c}}$ e velocidade $\mathrm{v}_{\mathrm{c}}$ para $\phi=10^{5} \mathrm{~N} / \mathrm{m}^{3}, \mathrm{~T}_{0, T D P=40 \mathrm{kN}}, \xi=20 \%$, $c_{0}=9.35991, T=4.5 s$ para $x_{c, 0}=0.1$ e $v_{c, 0}=0.07$
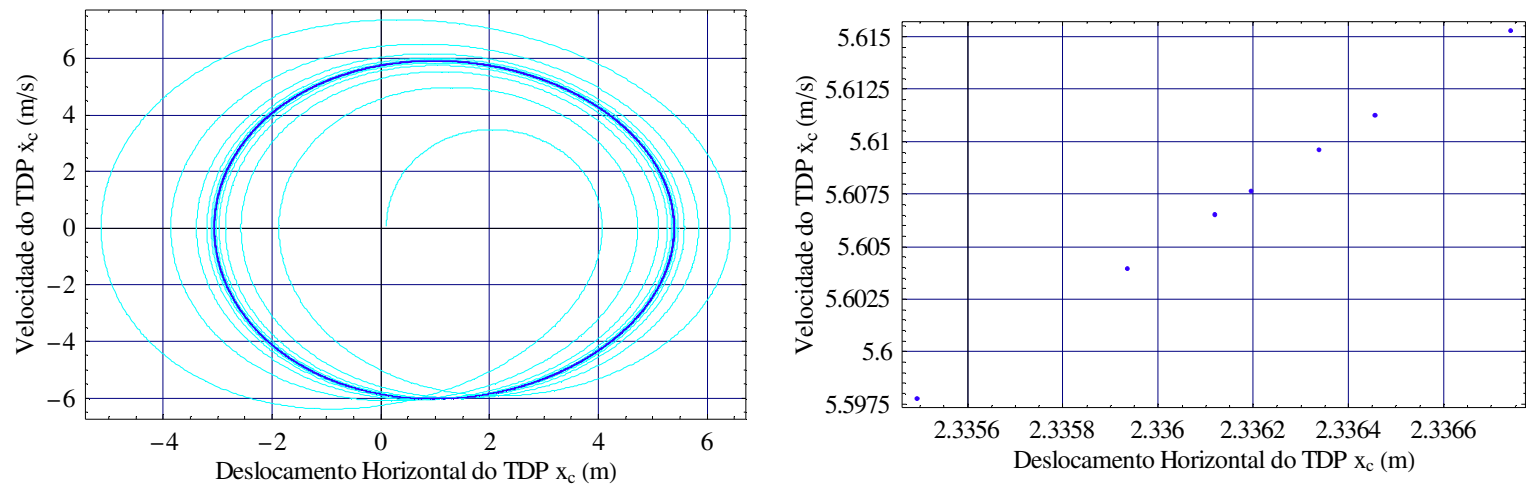

Figura 81: Mapa de fase e mapa de Poincaré para $\phi=10^{5} \mathrm{~N} / \mathrm{m}^{3}, \mathrm{~T}_{0, T D P}=40 \mathrm{kN}, \xi=20 \%, \mathrm{c}_{0}=9.35991, \mathrm{~T}=4.5 \mathrm{~s}$ para $x_{c, 0}=0.1$ e $v_{c, 0}=0.07$ 


\section{Equação do modelo de ordem reduzida para o caso P12:}

$$
\begin{aligned}
\ddot{U}+0.050217 \dot{U}+ & {[0.007004-0.000993 \cos (0.167391 t)] U } \\
& =0.014103 \cos (0.167391 t)+[0.003943+0.000366 \cos (0.167391 t)] U^{2} \\
& -0.027897 \dot{U}^{2}-0.448541 U \ddot{U}+[0.000423+0.000033 \cos (0.167391 t)] U^{3} \\
& +0.015356 U \dot{U}^{2}-0.075125 U^{2} \ddot{U}-0.000012 U^{4}+0.004862 U^{2} \dot{U}^{2}-0.005565 U^{3} \ddot{U} \\
& -0.000001 U^{5}+0.000314 U^{3} \dot{U}^{2}-0.000153 U^{4} \ddot{U}
\end{aligned}
$$
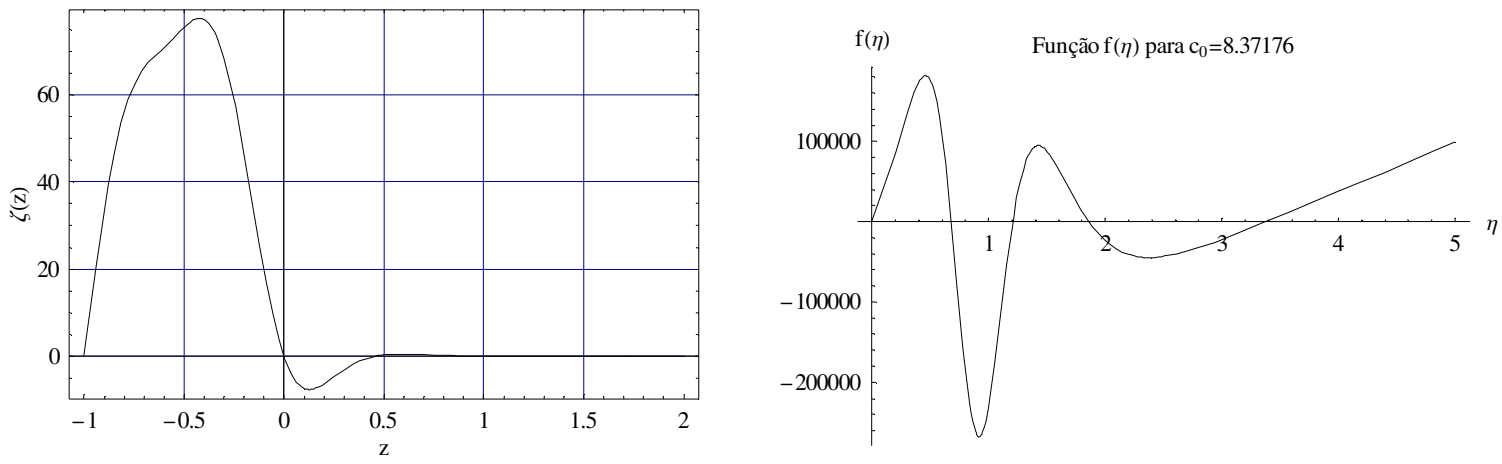

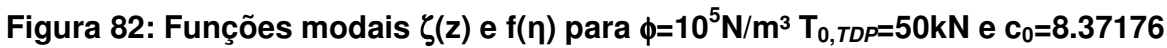
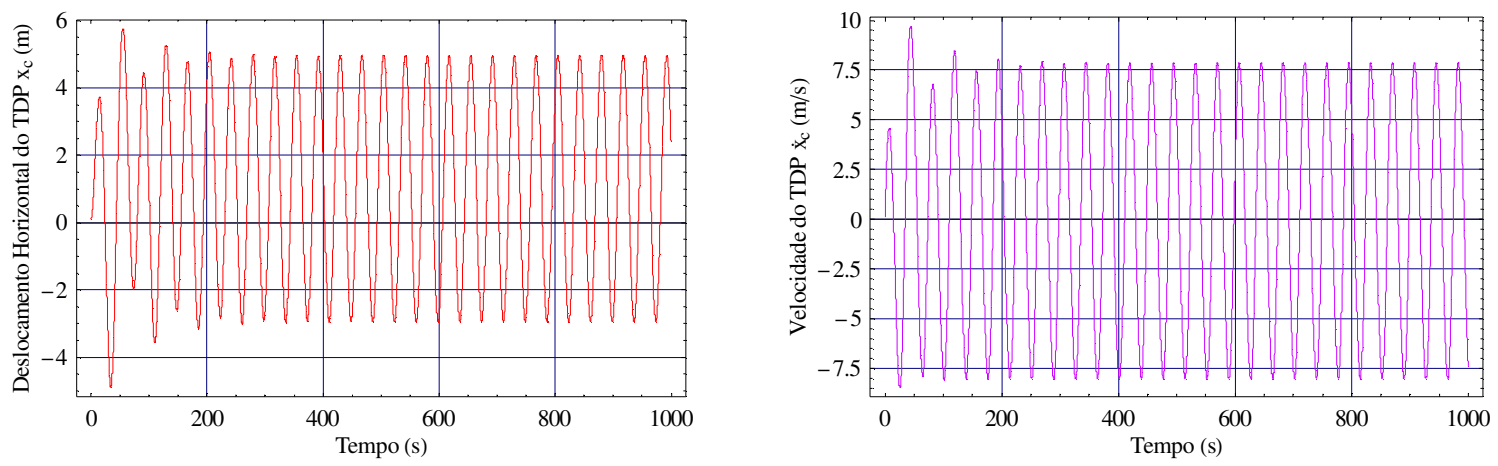

Figura 83: Deslocamento horizontal do $T D P \mathrm{x}_{\mathrm{c}}$ e velocidade $\mathrm{v}_{\mathrm{c}}$ para $\phi=10^{5} \mathrm{~N} / \mathrm{m}^{3}, \mathrm{~T}_{0, T D P=50 \mathrm{kN}}, \xi=20 \%$, $C_{0}=8.37176, T=3.1 s$ para $x_{c, 0}=0.1$ e $v_{c, 0}=0.1$
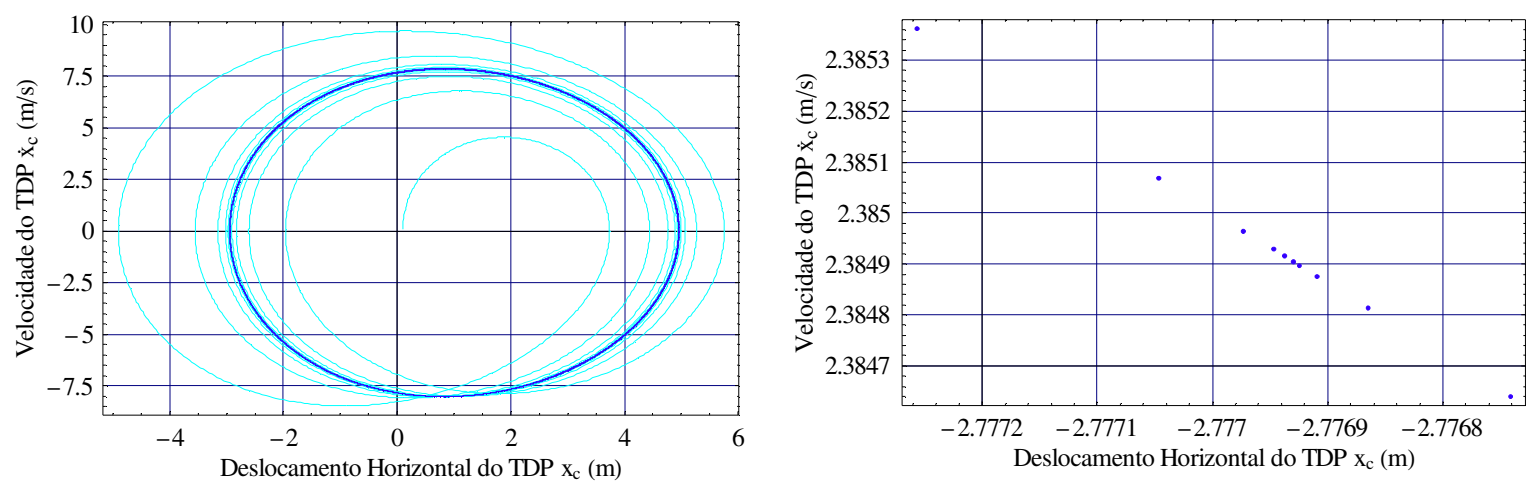

Figura 84: Mapa de fase e mapa de Poincaré para $\phi=10^{5} \mathrm{~N} / \mathrm{m}^{3}, \mathrm{~T}_{0, T D P=50 \mathrm{kN}}, \xi=20 \%, \mathrm{C}_{0}=8.37176, \mathrm{~T}=3.1 \mathrm{~s}$ para $x_{c, 0}=0.1$ e $v_{c, 0}=0.1$ 
As tabelas abaixo resumem os deslocamentos e velocidades do TDP obtidos para os processamentos $\mathrm{P} 1$ a $\mathrm{P} 6$, com taxa de amortecimento equivalente $\xi=10 \%$, e P7 a P12, com $\xi=30 \%$ :

\begin{tabular}{|c|c|c|c|c|c|c|}
\hline Caso & P1 & P2 & P3 & P4 & P5 & P6 \\
\hline$\varphi\left(N / m^{3}\right)$ & $10^{4}$ & $10^{4}$ & $10^{4}$ & $10^{4}$ & $10^{5}$ & $10^{5}$ \\
\hline$T_{0, T D P}(k N)$ & 20 & 30 & 40 & 50 & 40 & 50 \\
\hline Período (s) & 10.2 & 8.1 & 4.1 & 3.0 & 4.5 & 3.1 \\
\hline Taxa de amortecimento $\xi(\%)$ & 10 & 10 & 10 & 10 & 10 & 10 \\
\hline Deslocamento inicial $x_{c, 0}(m)$ & 0.1 & 0.1 & 0.1 & 0.1 & 0.1 & 0.1 \\
\hline Velocidade inicial $v_{c, 0}(m / s)$ & 0.03 & 0.04 & 0.08 & 0.10 & 0.07 & 0.10 \\
\hline Deslocamento estacionário $x_{c, e s t}(m)$ & 75.0 & 20.0 & 18.0 & 14.0 & 22.0 & 13.0 \\
\hline Velocidade estacionário $v_{c, \text { est }}(\mathrm{m} / \mathrm{s})$ & 20.0 & 15.0 & 16.0 & 17.0 & 18.0 & 17.0 \\
\hline Deslocamento máximo $x_{c, \max }(m)$ & 75.0 & 20.0 & 18.0 & 16.0 & 22.0 & 15.0 \\
\hline Velocidade máxima $v_{c, \max }(\mathrm{m} / \mathrm{s})$ & 20.0 & 15.0 & 16.0 & 17.0 & 18.0 & 17.0 \\
\hline
\end{tabular}

Tabela 8: Resumo dos processamentos $\mathrm{P} 1$ a P6, com $\xi=10 \%$, na aplicação do modelo a risers em catenária

\begin{tabular}{|c|c|c|c|c|c|c|}
\hline Caso & P6 & P7 & P8 & P9 & $P 10$ & P11 \\
\hline$\varphi\left(N / m^{3}\right)$ & $10^{4}$ & $10^{4}$ & $10^{4}$ & $10^{4}$ & $10^{5}$ & $10^{5}$ \\
\hline$T_{0, T D P}(k N)$ & 20 & 30 & 40 & 50 & 40 & 50 \\
\hline Período (s) & 10.17 & 8.14 & 4.07 & 3.01 & 4.45 & 3.13 \\
\hline Taxa de amortecimento $\xi(\%)$ & 30 & 30 & 30 & 30 & 30 & 30 \\
\hline Deslocamento inicial $x_{c, o}(m)$ & 0.1 & 0.1 & 0.1 & 0.1 & 0.1 & 0.1 \\
\hline Velocidade inicial $v_{c, 0}(m / s)$ & 0.03 & 0.04 & 0.08 & 0.10 & 0.07 & 0.10 \\
\hline Deslocamento estacionário $x_{c, e s t}(m)$ & 9.0 & 16.0 & 4.5 & 4.0 & 5.0 & 5.0 \\
\hline Velocidade estacionário $v_{c, \text { est }}(\mathrm{m} / \mathrm{s})$ & 4.5 & 9.0 & 5.5 & 6.5 & 6.0 & 8.0 \\
\hline Deslocamento máximo $x_{c, \max }(m)$ & 11.0 & 17.0 & 5.5 & 5.0 & 6.0 & 6.0 \\
\hline Velocidade máxima $v_{c, \max }(\mathrm{m} / \mathrm{s})$ & 5.5 & 11.0 & 7.0 & 8.0 & 7.0 & 10.0 \\
\hline
\end{tabular}

Tabela 9: Resumo dos processamentos P7 a P12, com $\xi=30 \%$, na aplicação do modelo a risers em catenária 
Note-se que para os casos P1 a P4, e P7 a P10, utilizou-se o mesmo coeficiente de rigidez do solo $\varphi=10^{4} \mathrm{~N} / \mathrm{m}^{3}$, variando-se apenas a tração estática $T_{0, T D P}$ no TDP para cada caso, e para os casos P5, P6, P11 e P12 utilizou-se o mesmo coeficiente de rigidez do solo $\varphi=10^{5} \mathrm{~N} / \mathrm{m}^{3}$. Considera-se que valores menores para a rigidez do solo são atípicos em situações reais, o que contextualiza o valor utilizado em relação aos valores existentes na bibliografia, como em Takafugi [39]. Para valores maiores da rigidez do solo $\varphi$, os períodos naturais da viga tendem a ser muito pequenos, da ordem de centésimos de segundo, o que pode implicar dificuldade de realização da condição de ressonância paramétrica, já que a excitação teria que ter período igual à metade destes valores, já muito baixos.

Os gráficos das simulações numéricas e as Tab. 8 e 9 permitem observar que os valores para deslocamentos tiveram ampliação em todos os casos, a partir do deslocamento inicial de $0.1 \mathrm{~m}$, comum a todos os casos.

Note-se que nos casos P1 a P6, menor taxa de amortecimento levou a deslocamentos maiores, enquanto que nos casos P7 a P12, maior taxa de amortecimento levou a deslocamentos menores.

Nota-se também uma pequena alteração quanto à forma do diagrama de fase, para os casos P7 a P12 frente aos casos P1 a P6. Isso se deve essencialmente à relação taxa de amortecimento equivalente $\xi$ e tração dinâmica no ponto "O", $T_{1}$.

Em todos os casos a solução convergiu para um atrator periódico. Não foram constatados padrões típicos de impacto, nem de pulsos ("traveling waves"), ao menos para os cenários estudados nestes casos.

Observa-se que os deslocamentos são maiores para casos em que a tração estática no ponto "O" é mais baixa. Isso se deve ao fato que aumento da tração estática no ponto "O" aumenta a rigidez da linha, fazendo com que os deslocamentos dinâmicos tendam a ser mais baixos. 
As equações para os diferentes modelos de ordem reduzida permitem observar que os coeficientes associados às não linearidades quadráticas e cúbicas são preponderantes. Os demais coeficientes das equações são menos representativos, mesmo que não sejam desprezíveis.

Em relação aos mapas de Poincaré, observa-se nos casos P1 a P6 que a solução tem período dois. Nos casos P7 a P12 há solução de período um. Na maioria dos casos nota-se ainda um pequeno efeito do transiente. 


\subsection{Pós-processamento: obtenção dos esforços solicitantes}

A partir da integração da equação de movimento do modelo é possível fazer o caminho inverso àquele que levou à adimensionalização do problema, de forma a obter a solução completa, seguindo os seguintes passos: $u(z, \tau)=\hat{u}(z)+\delta(z, \tau)$, sendo $\delta(z, \tau)=\zeta(z) \cdot U(\tau)$, onde $\zeta(z)$ é a função modal descrita em (75) e (76), e $U(\tau)$ é a variável modal. A partir da relação $w(x, t)=\left(\frac{p}{\mu}\right) u(z, \tau)$, sendo $p$ o peso submerso do riser e $\mu$ o coeficiente de rigidez do solo por unidade de área, e da transformação de coordenadas $x=(z+1) \frac{c(\tau)}{\alpha}$, sendo $c(\tau)=c_{0}+U(\tau)$ a posição do TDP e $t=\frac{\tau}{\beta}$, chega-se à solução completa para o caso P3, descrito na seção 4.2, utilizado como exemplo.

De maneira similar, através das relações $M(x, t)=-E I \frac{\partial^{2} w}{\partial x^{2}}(x, t) \quad \mathrm{e}$ $T(x, t)=\left[T_{0}+T_{1} \cos \left(\Omega_{d} t\right)\right] e^{-a x}, T(x, t)$ definido em (91) e (92) considerando (82), e $\sigma(x, t)=\frac{E I}{W} \frac{\partial^{2} w}{\partial x^{2}}(x, t)+\frac{T(x, t)}{A}$, sendo $W$ o módulo de resistência do riser $\mathrm{e}$ $\frac{\partial^{2} w}{\partial x^{2}}(x, t)=\left(\frac{p \alpha^{2}}{\mu}\right) \frac{\partial^{2} u}{\partial z^{2}}(z, \tau)$, com $\alpha$ definido em (3), determinam-se os esforços de momento fletor, força normal e máxima tensão normal para cada abscissa em um determinado instante. Analogamente, através das relações $V(x, t)=-E I \frac{\partial^{3} w}{\partial x^{3}}(x, t)$ e $\tau(x, t)=-\frac{4}{3} \frac{E I}{A} \frac{\partial^{3} w}{\partial x^{3}}(x, t)+\frac{1}{2 e} \frac{\partial T}{\partial x}(x, t)$ sendo $A$ a seção transversal, $\frac{\partial^{3} w}{\partial x^{3}}(x, t)=\left(\frac{p \alpha^{3}}{\mu}\right) \frac{\partial^{3} u}{\partial z^{3}}(z, \tau)$, e $e$ a metade da espessura do núcleo elástico da seção, determinam-se os esforços de força cortante e máxima tensão cisalhante. 
Cada uma das cores nos gráficos desta seção representa um instante no tempo, sendo a extremidade suspensa o ponto "O".

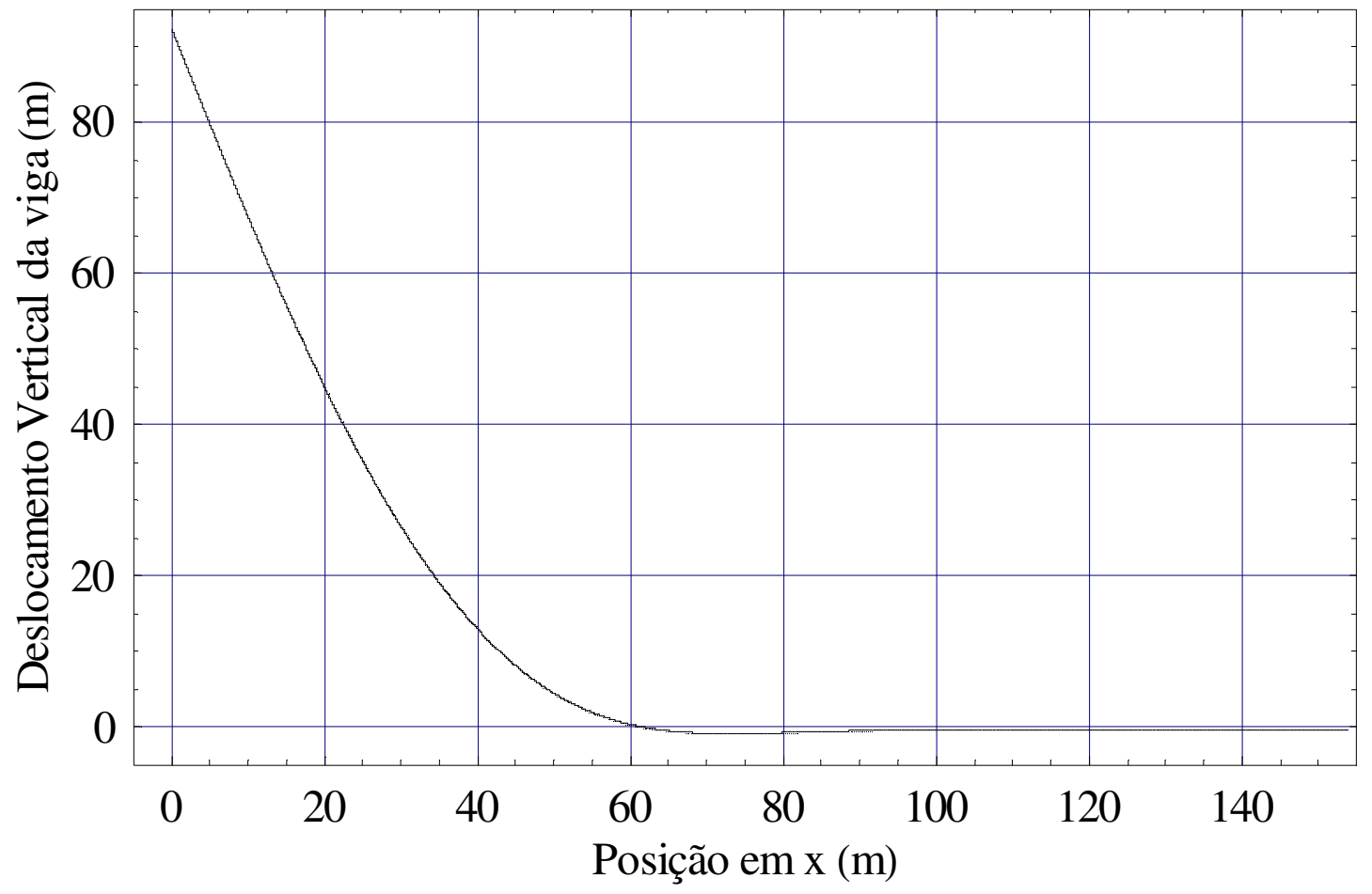

Figura 85: Configuração estática

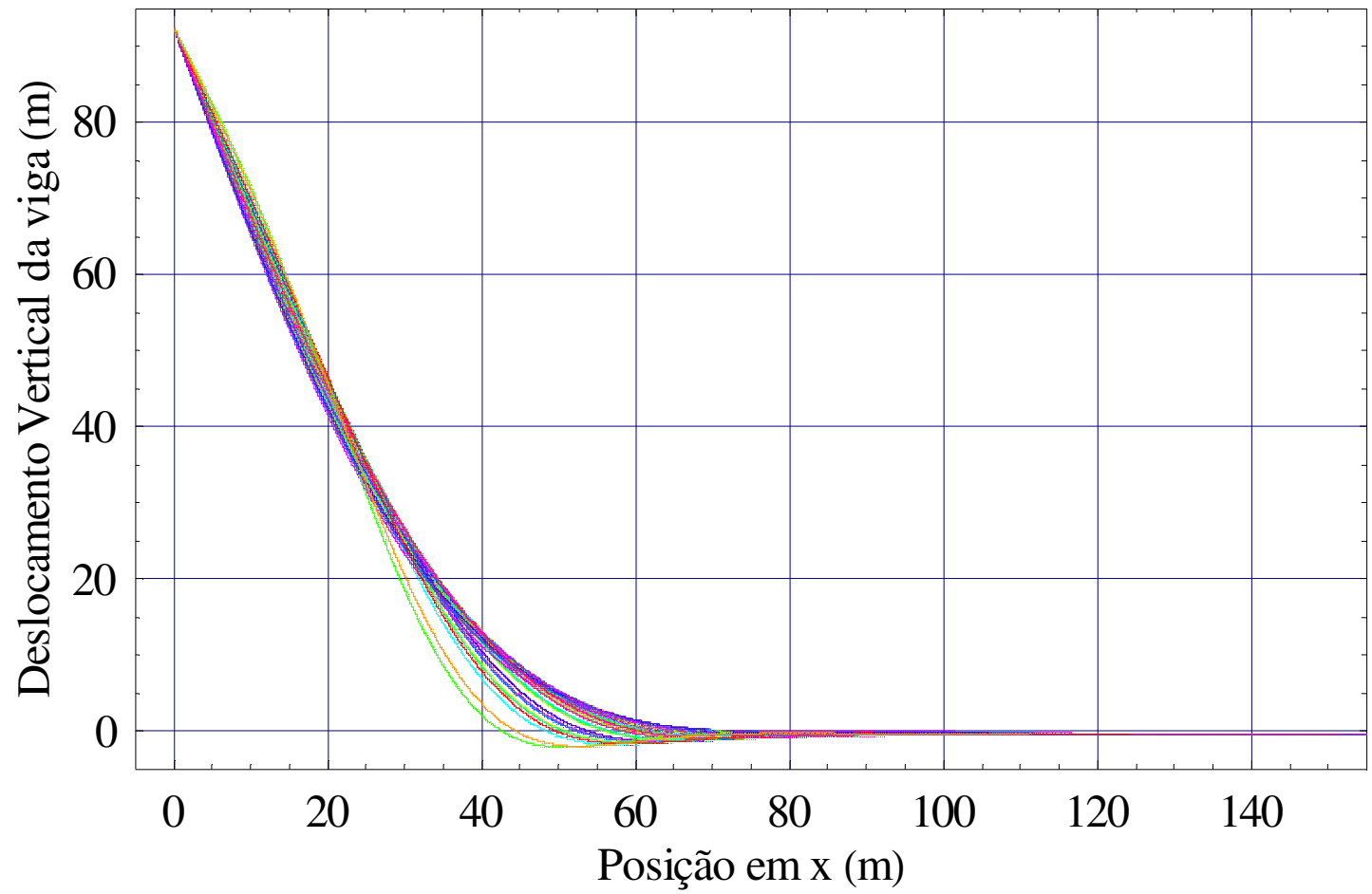

Figura 86: Solução completa em diferentes instantes 


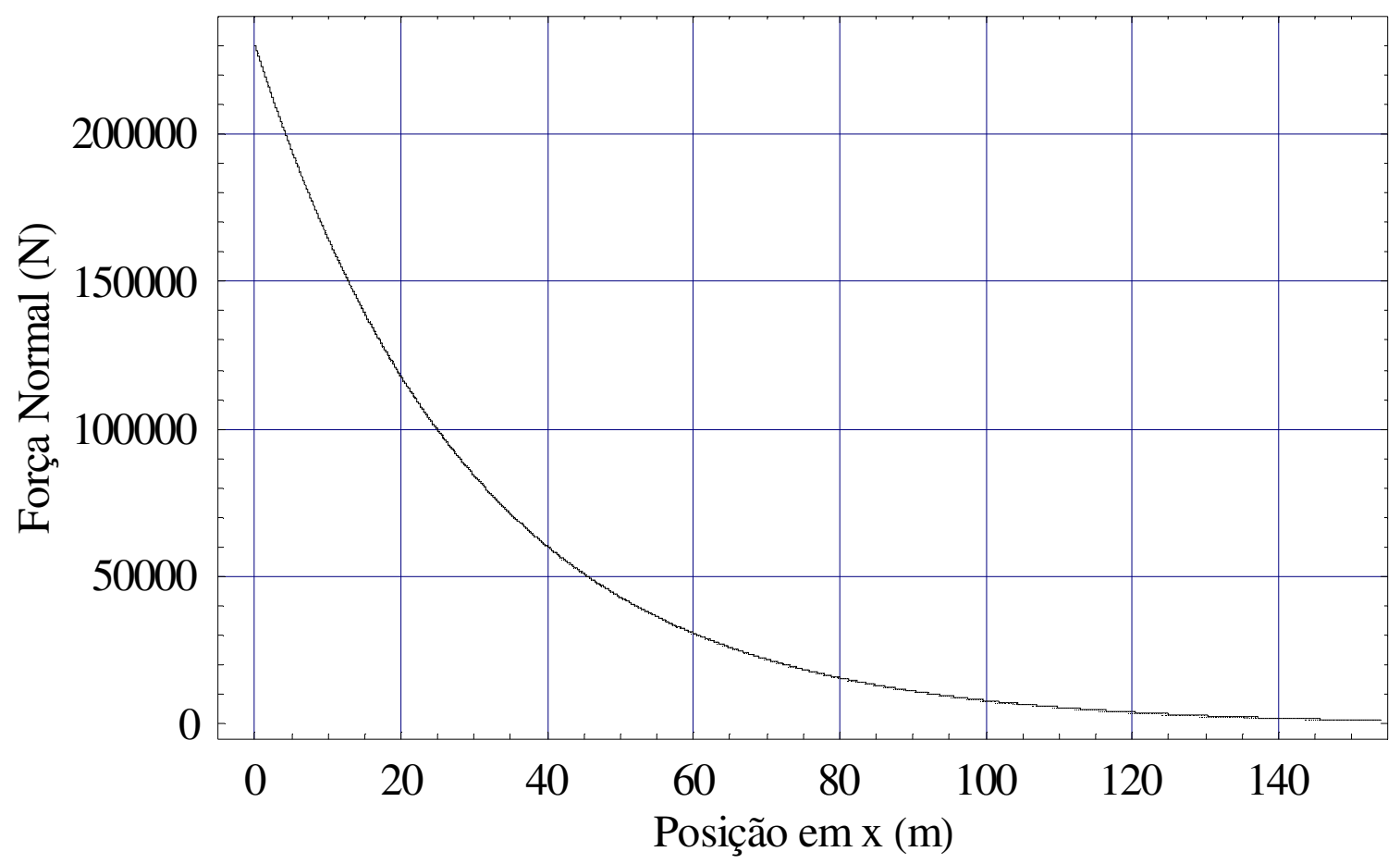

Figura 87: Força Normal na configuração estática (N)

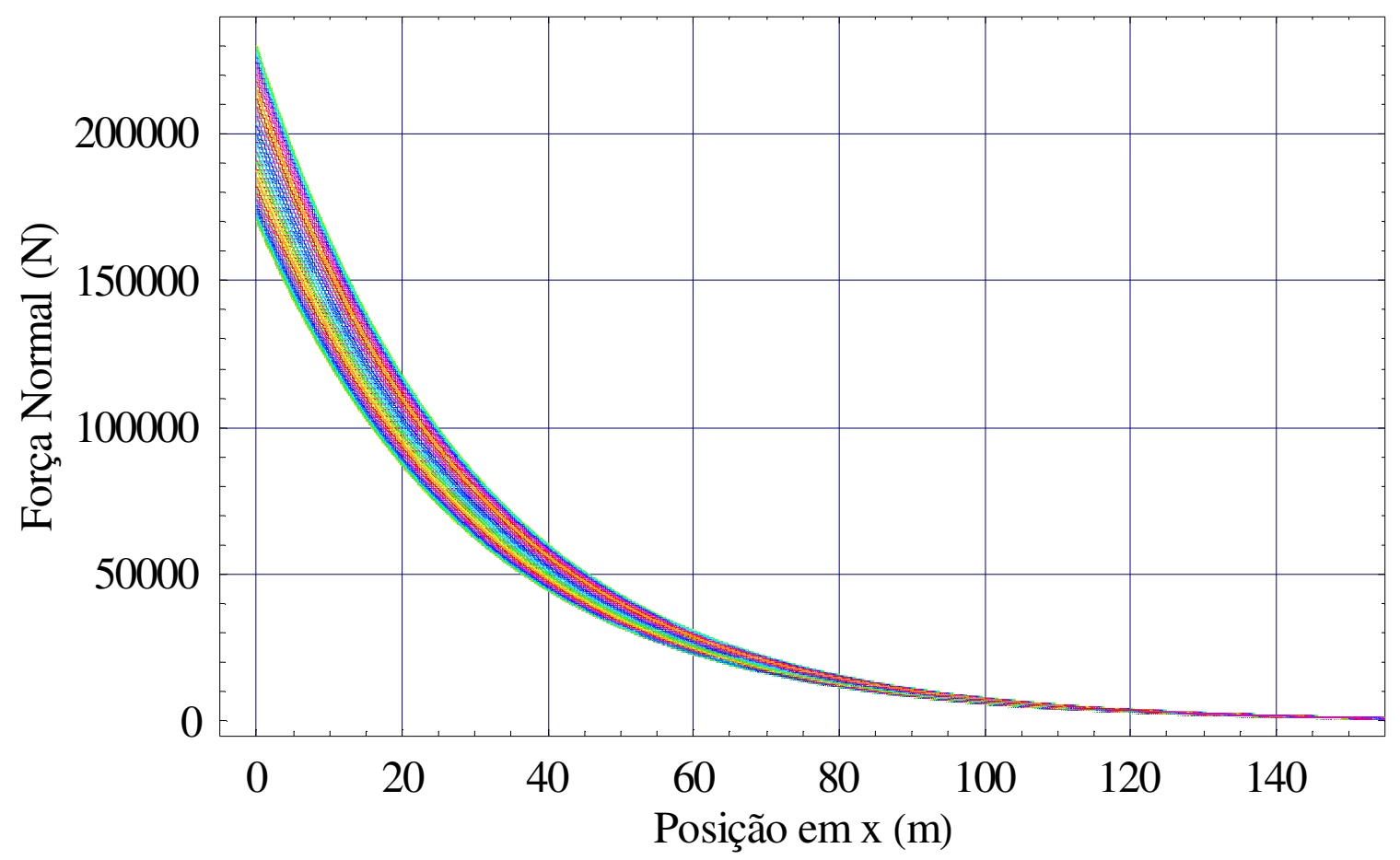

Figura 88: Força Normal em diferentes instantes (N) 


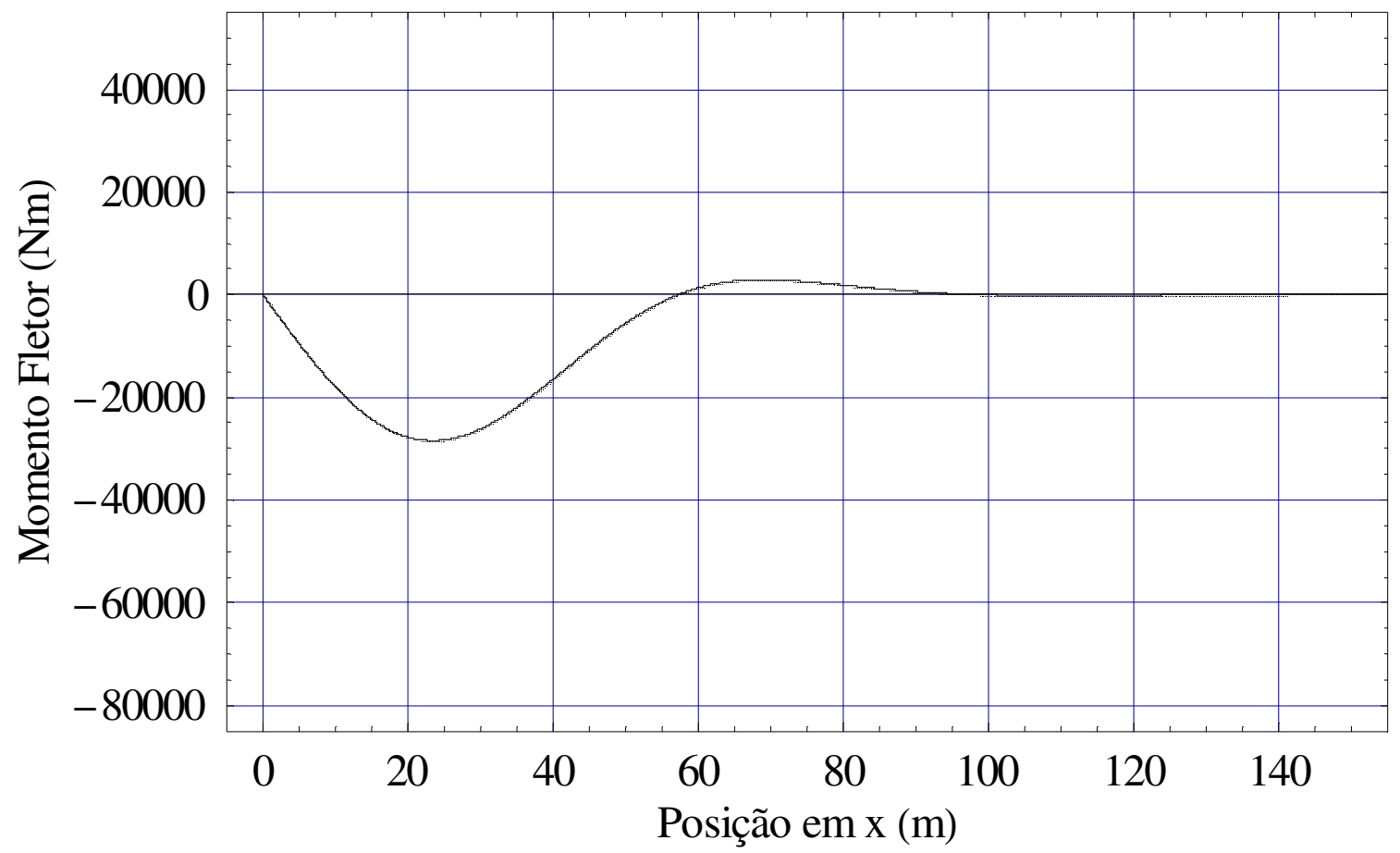

Figura 89: Momento Fletor na configuração estática (Nm)

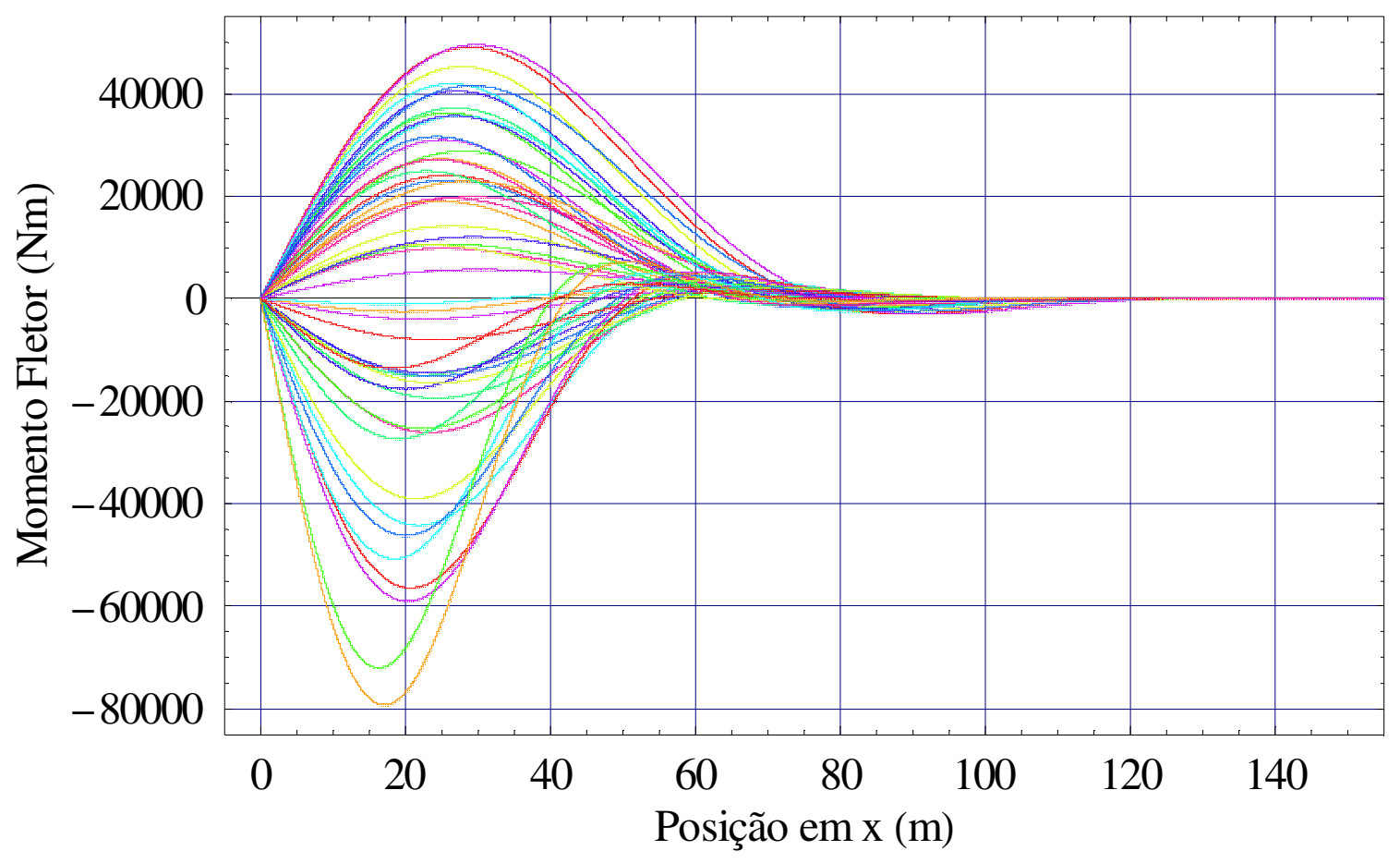

Figura 90: Momento Fletor em diferentes instantes (Nm) 


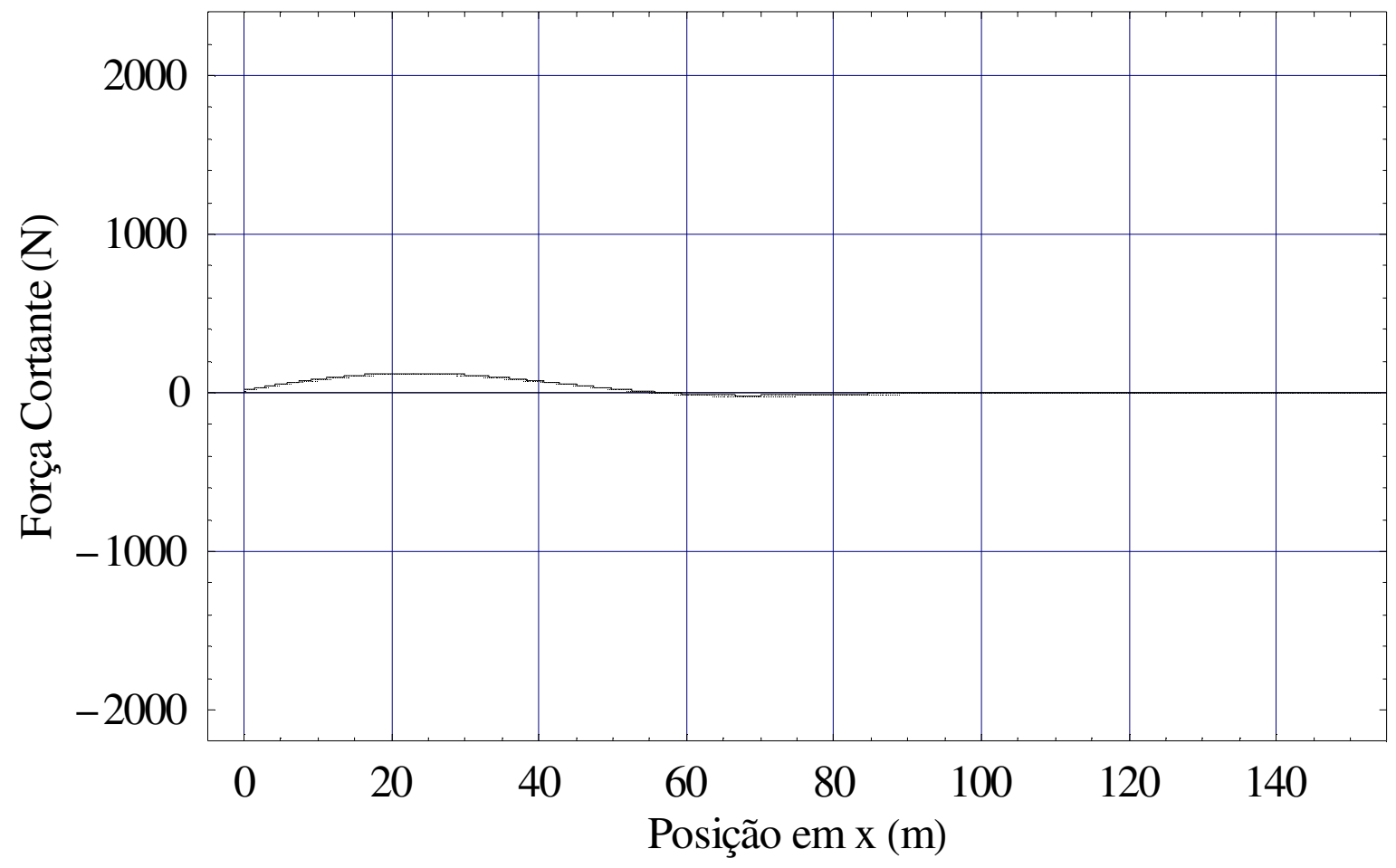

Figura 91: Força Cortante na configuração estática (N)

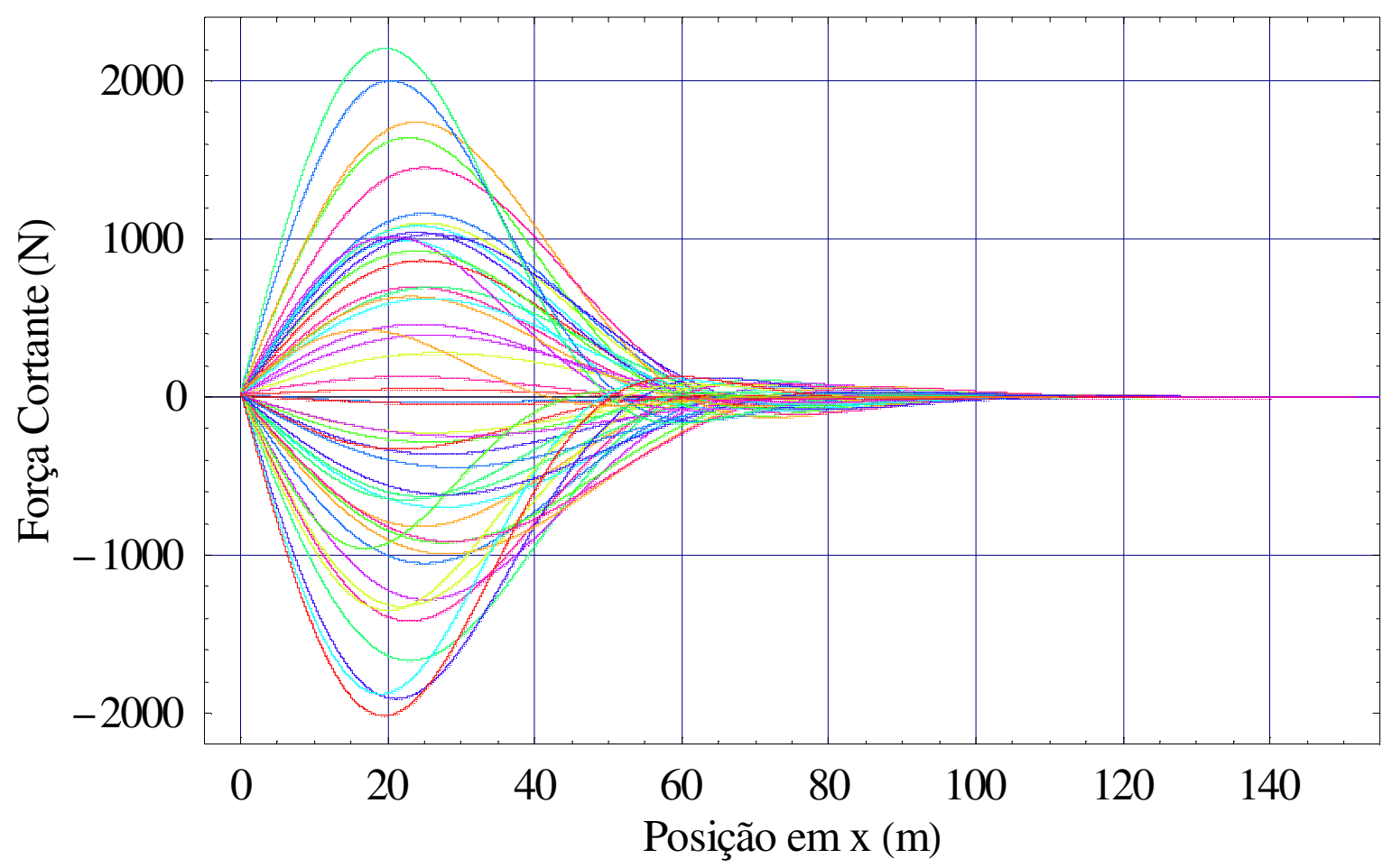

Figura 92: Força Cortante em diferentes instantes (N) 


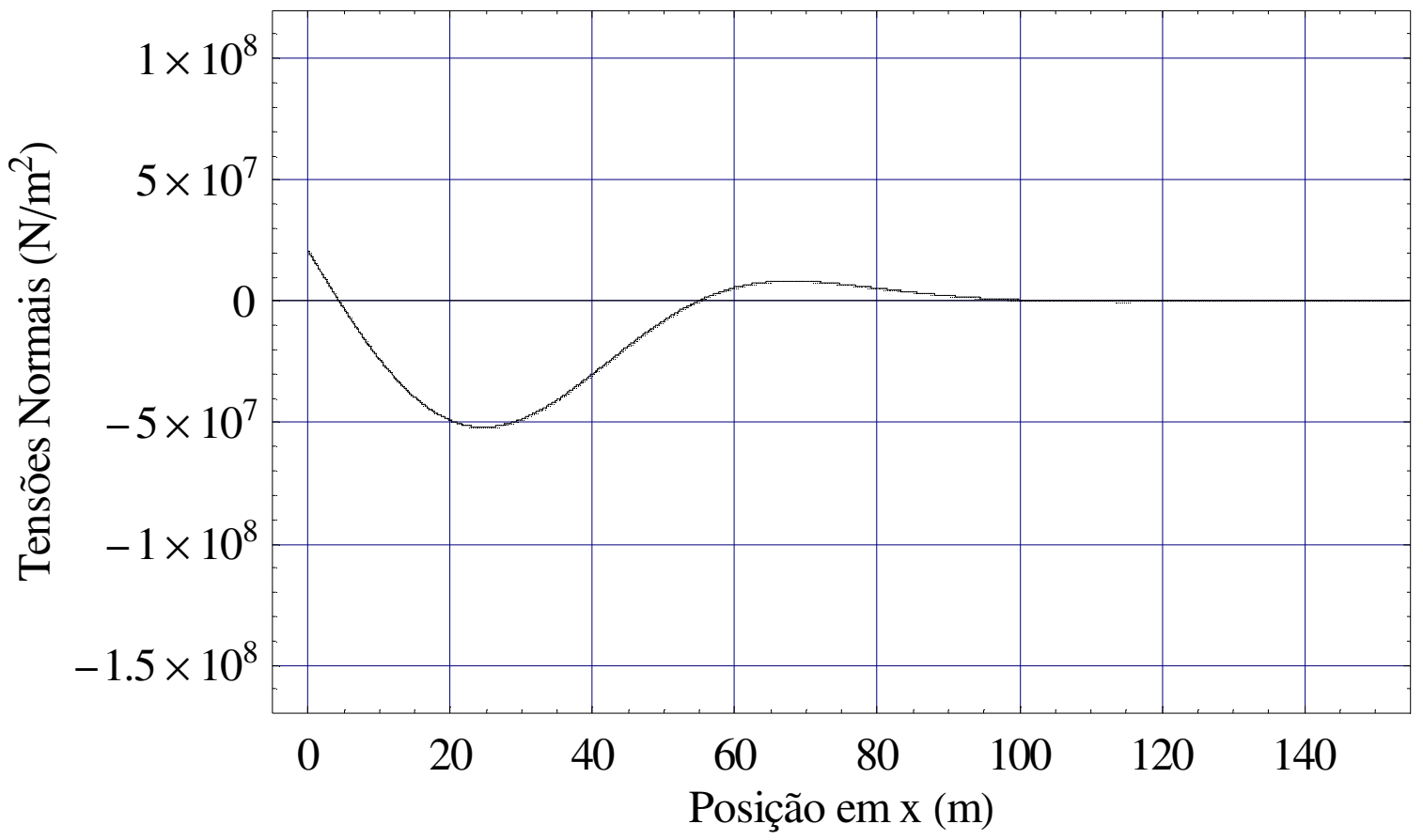

Figura 93: Tensões Normais na configuração estática $\left(\mathrm{N} / \mathrm{m}^{2}\right)$

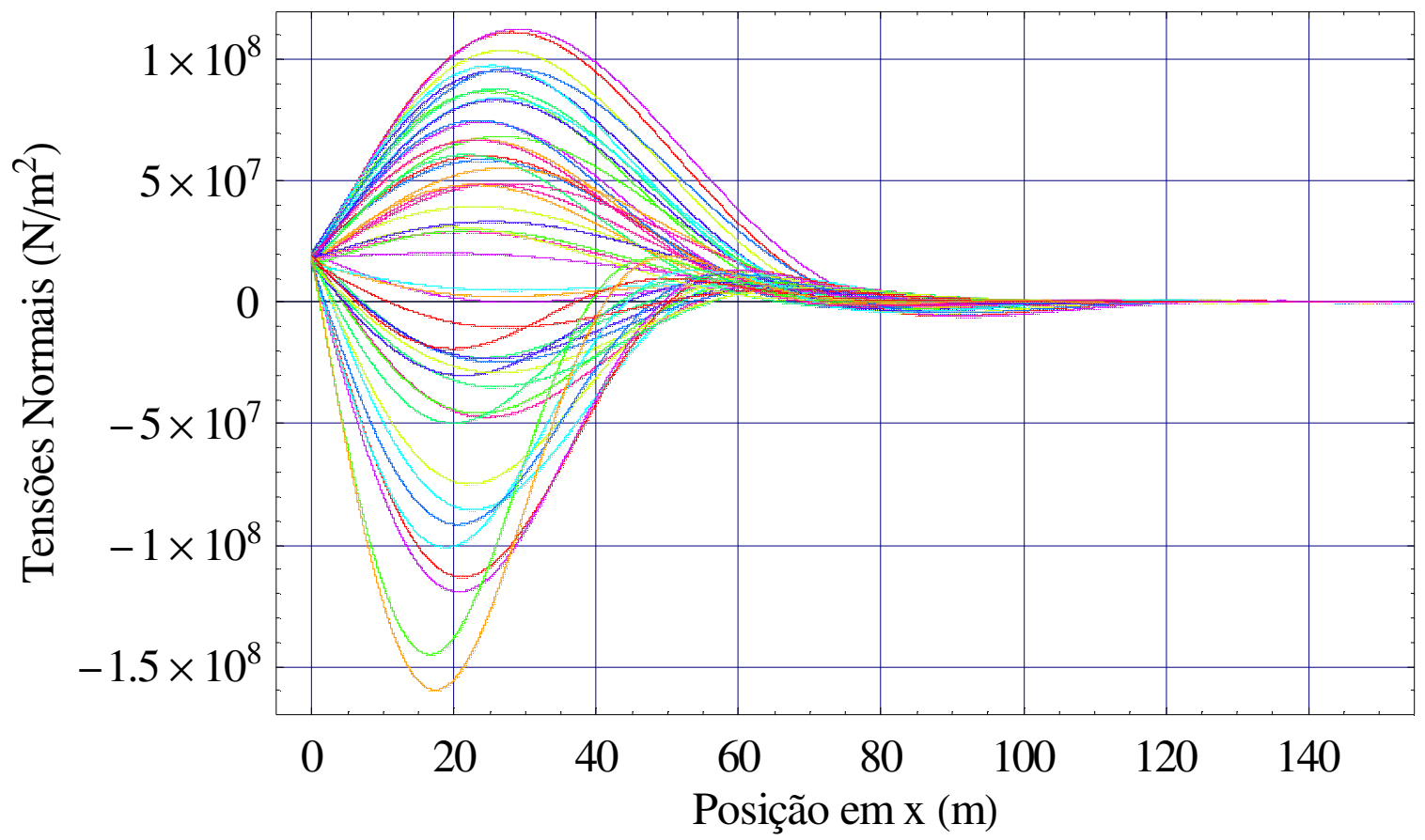

Figura 94: Tensões Normais em diferentes instantes $\left(\mathrm{N} / \mathrm{m}^{2}\right)$ 


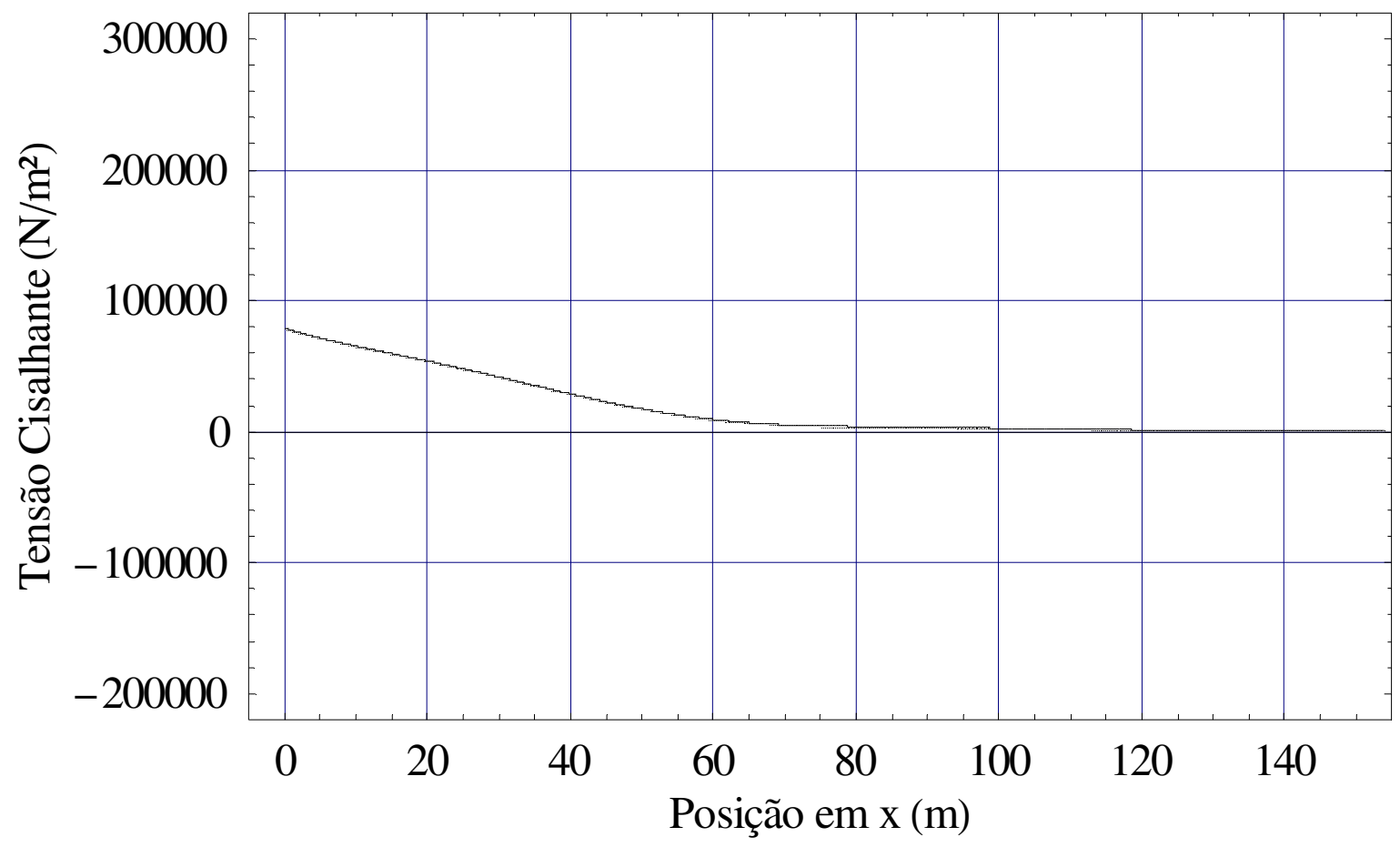

Figura 95: Tensão Cisalhante na configuração estática $\left(\mathrm{N} / \mathrm{m}^{2}\right)$

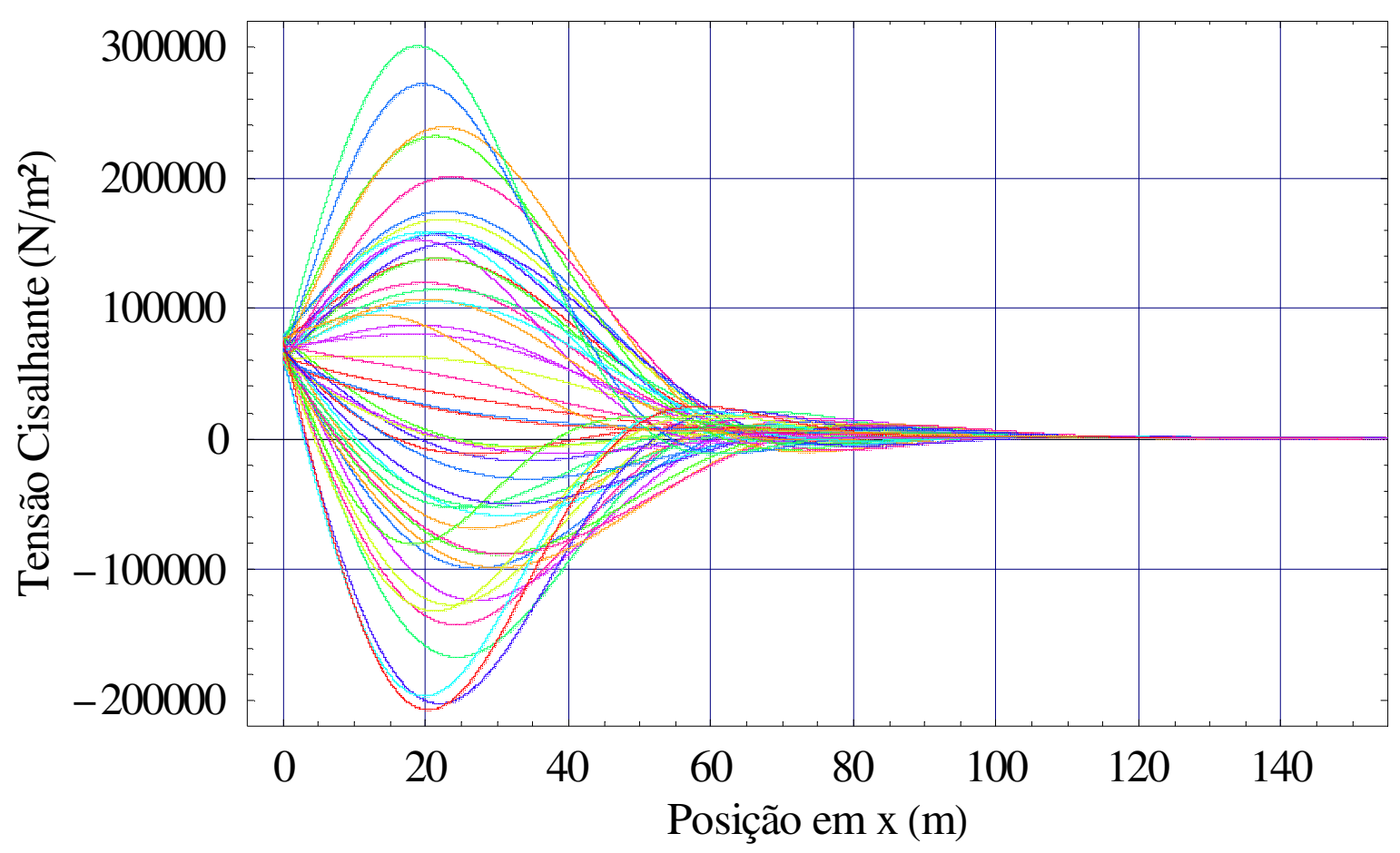

Figura 96: Tensão Cisalhante em diferentes instantes $\left(\mathrm{N} / \mathrm{m}^{2}\right)$ 
Assim, mostra-se que, a partir da integração da equação de movimento do modelo, é possível obter os esforços solicitantes para o riser, na região do TDZ.

É possível observar também que os esforços são sempre majorados na configuração dinâmica, 3 vezes maior no caso das tensões normais e 4 vezes no caso da tensão cisalhante.

Recorde-se que a tração foi adotada no modelo de forma "ad hoc", baseando-se nas premissas de cálculo discutidas no capítulo 2 e verificadas no anexo 7.3.

A Fig. 86 mostra as diferentes posições em que o riser toca o solo, evidenciando assim a recuperação do movimento do TDP no eixo das abscissas.

Nota-se que os esforços oriundos da flexão são preponderantes frente aos esforços de cisalhamento.

A análise dos gráficos desta seção se mostra uma importante ferramenta na avaliação de esforços de fadiga, e que pode contribuir, inclusive, na determinação da vida útil de um riser. 


\subsection{Comparação dos resultados obtidos com o programa Poliflex 3D: casos sem ressonância paramétrica}

A partir dos resultados obtidos nas seções anteriores 4.1. e 4.2., pretendese fazer uma comparação entre os resultados dos processamentos obtidos com os programas Poliflex 3D, nesta seção, e Orcaflex, na seção 4.5., com o intuito de avaliar as características principais de cada uma das rotinas de processamento sugeridas.

Para comparação, foram escolhidas três configurações de riser, processados tanto no programa Poliflex 3D quanto no modelo apresentado neste trabalho. Verificou-se através do modelo que tais configurações possuem período associado à freqüência natural baixos, de forma que, dadas as condições de mar usuais, a ocorrência de ressonância paramétrica torna-se pouco provável.

Os dados de entrada para os três processamentos, utilizados tanto no programa Poliflex 3D quanto no modelo aqui proposto, podem ser verificados na tabela abaixo:

\begin{tabular}{|c||c|c|c|}
\hline Caso & $\mathbf{1}$ & $\mathbf{2}$ & $\mathbf{3}$ \\
\hline \hline $\boldsymbol{\varphi}_{\boldsymbol{i}}\left(\mathbf{N} / \mathbf{m}^{\mathbf{3}}\right)$ & $10^{5}$ & $10^{5}$ & $10^{5}$ \\
\hline $\boldsymbol{T}_{\text {o,TDP }}(\mathbf{k N})$ & 495,1 & 324,7 & 125,0 \\
\hline $\boldsymbol{T}_{\text {TREM DE ONDAS }}(\mathbf{s})$ & 11 & 11 & 11 \\
\hline
\end{tabular}

Tabela 10: Dados de entrada para comparação com programa Poliflex 3D

A seguir, apresenta-se o diagrama de fase de cada um dos processamentos no modelo proposto neste trabalho, com dados de entrada da Tab. 10: 


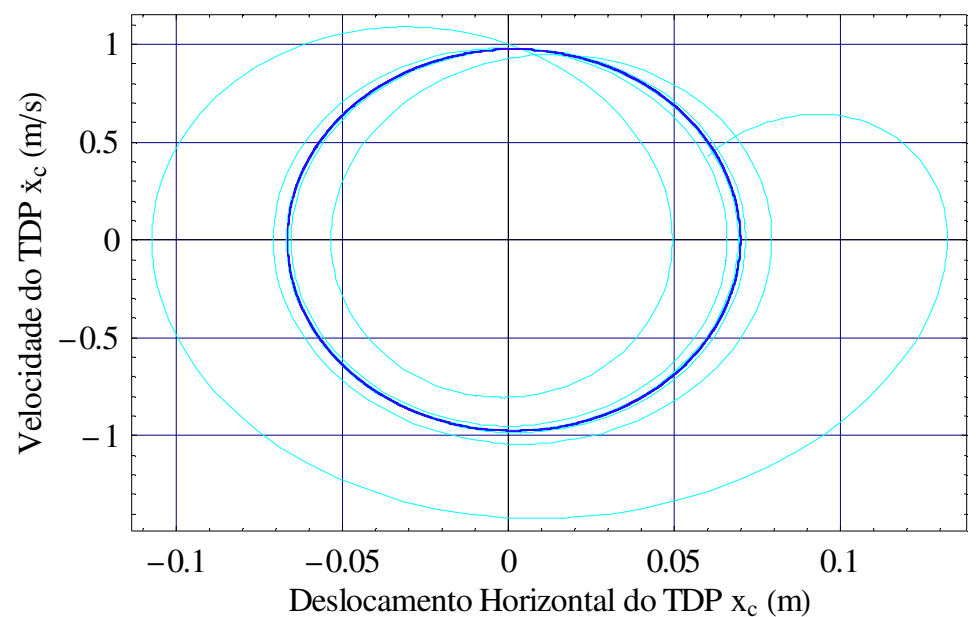

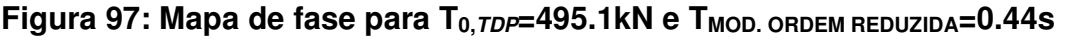

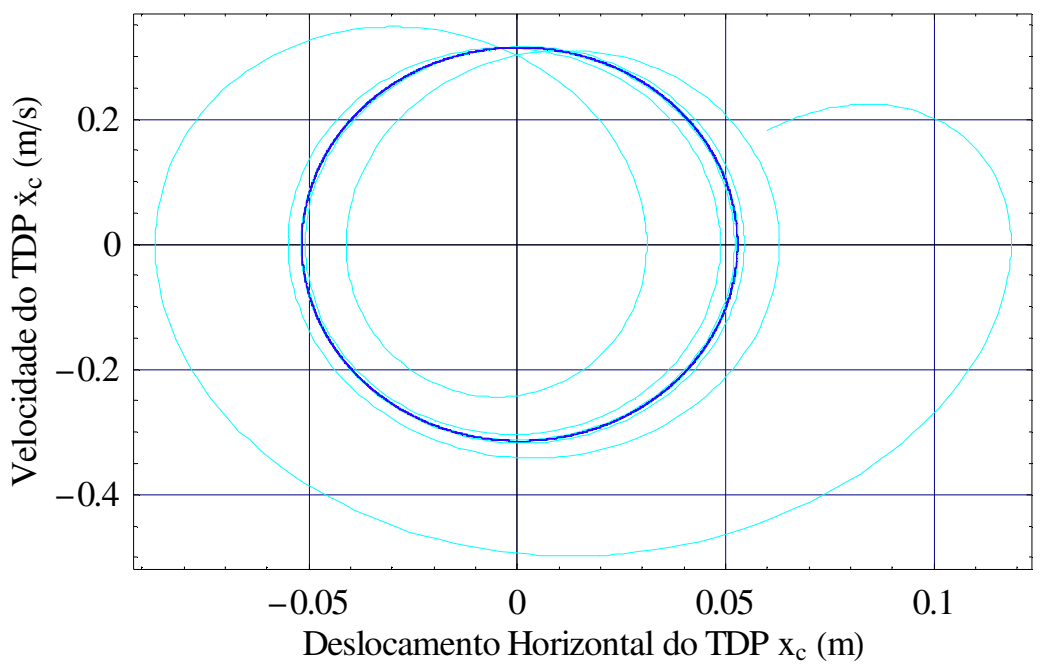

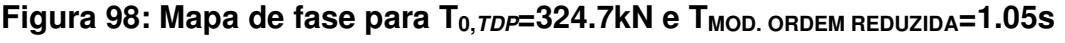

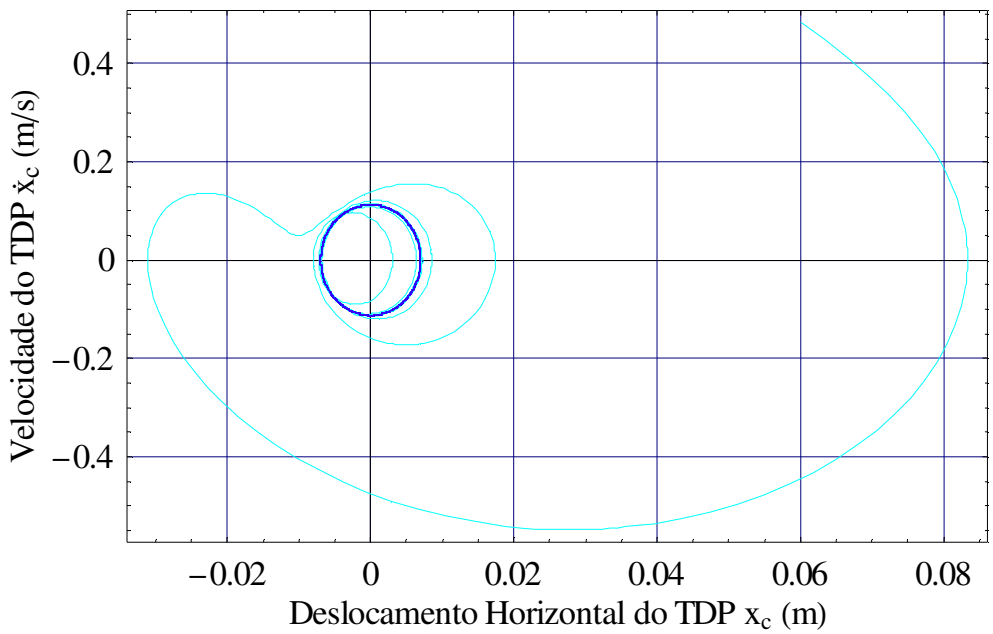

Figura 99: Mapa de fase para $T_{0, T D P}=125.0 \mathrm{kN}$ e $\mathrm{T}_{\text {MOD. ORDEM REDUZIDA }}=0.40 \mathrm{~s}$ 
Os resultados dos processamentos segundo o modelo aqui proposto podem ser resumidos na Tab. 11 abaixo:

\begin{tabular}{|c||c|c|c|}
\hline Caso & $\mathbf{1}$ & $\mathbf{2}$ & $\mathbf{3}$ \\
\hline \hline $\boldsymbol{X}_{\text {TDP }}(\boldsymbol{m})$ & 0.100 & 0.100 & 0.020 \\
\hline $\boldsymbol{v}_{\text {TDP }}(\mathbf{m} / \mathbf{s})$ & 2 & 0.6 & 0.2 \\
\hline $\boldsymbol{T}_{\text {MOD. ORDEM REDUZIDA }}(\boldsymbol{s})$ & 0.44 & 1.05 & 0.40 \\
\hline
\end{tabular}

Tabela 11: Resultados do processamento sem excitação paramétrica

Para os mesmos valores de entrada da Tab. 10, os resultados obtidos após processamento com programa Poliflex 3D podem ser resumidos na Tab. 12 abaixo:

\begin{tabular}{|c||c|c|c|}
\hline Caso & $\mathbf{1}$ & $\mathbf{2}$ & $\mathbf{3}$ \\
\hline \hline $\boldsymbol{X}_{T D P}(\mathbf{m})$ & 0.06 & 0.011 & 0.017 \\
\hline
\end{tabular}

Tabela 12: Resultados do processamento com Poliflex 3D sem excitação paramétrica

Cabe aqui considerar que o programa Poliflex 3D não fornece informações quanto à velocidade, mas apenas ao deslocamento de cada ponto, motivo pelo qual a Tab. 12 mostra apenas os resultados de deslocamento.

Observa-se que os valores que constam como deslocamento inicial, $x_{0, T D P}$, são os valores de saída do programa Poliflex 3D, utilizados como perturbação inicial no modelo proposto.

Portanto, os processamentos mostraram que, nos casos em que não ocorre excitação paramétrica, os valores de deslocamento do TDP são da mesma ordem de grandeza, tanto no modelo aqui proposto como no programa Poliflex 3D. 


\subsection{Comparação dos resultados obtidos com o programa Orcaflex: casos com ressonância paramétrica}

De forma análoga ao que foi desenvolvido na seção 4.3., pretende-se agora comparar os resultados de processamentos no modelo apresentado no presente trabalho com o programa Orcaflex, para casos com ressonância paramétrica.

Para comparação, foram escolhidas três configurações de riser, processados tanto no programa Orcaflex quanto no modelo apresentado neste trabalho. Verificou-se através do modelo que tais configurações possuem período associado à freqüência natural similares ao dobro das condições de mar usuais, de forma que a ocorrência de ressonância paramétrica torna-se muito provável.

Os dados de entrada para os três processamentos, utilizados tanto no programa Orcaflex quanto no modelo aqui proposto, podem ser verificados na tabela abaixo:

\begin{tabular}{|c||c|c|c|}
\hline Caso & $\mathbf{1}$ & $\mathbf{2}$ & $\mathbf{3}$ \\
\hline \hline $\boldsymbol{\varphi}_{\boldsymbol{i}}\left(\mathbf{N} / \mathbf{m}^{\mathbf{3}}\right)$ & $10^{4}$ & $10^{4}$ & $10^{5}$ \\
\hline $\boldsymbol{T}_{\boldsymbol{0}, \text { TDP }}(\mathbf{k N})$ & 35 & 50 & 60 \\
\hline $\boldsymbol{T}_{\text {TREM DE ONDAS }}(\mathbf{s})$ & 6.2 & 3.0 & 3.3 \\
\hline
\end{tabular}

Tabela 13: Dados de entrada para comparação com programa Orcaflex

A seguir, o diagrama de fase de cada um dos processamentos no modelo proposto neste trabalho, com dados de entrada da Tab. 13: 


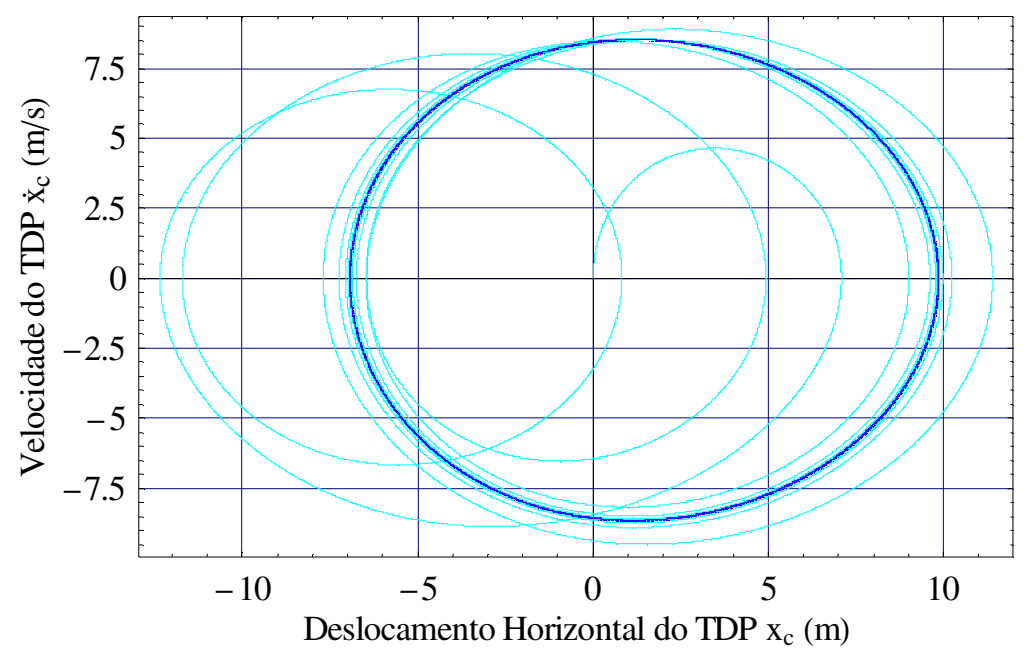

Figura 100: Mapa de fase para $T_{0, T D P=35 \mathrm{kN}}$ e $\mathrm{T}_{\text {MOD. ORDEM REDUZIDA }}=12.4 \mathrm{~s}$

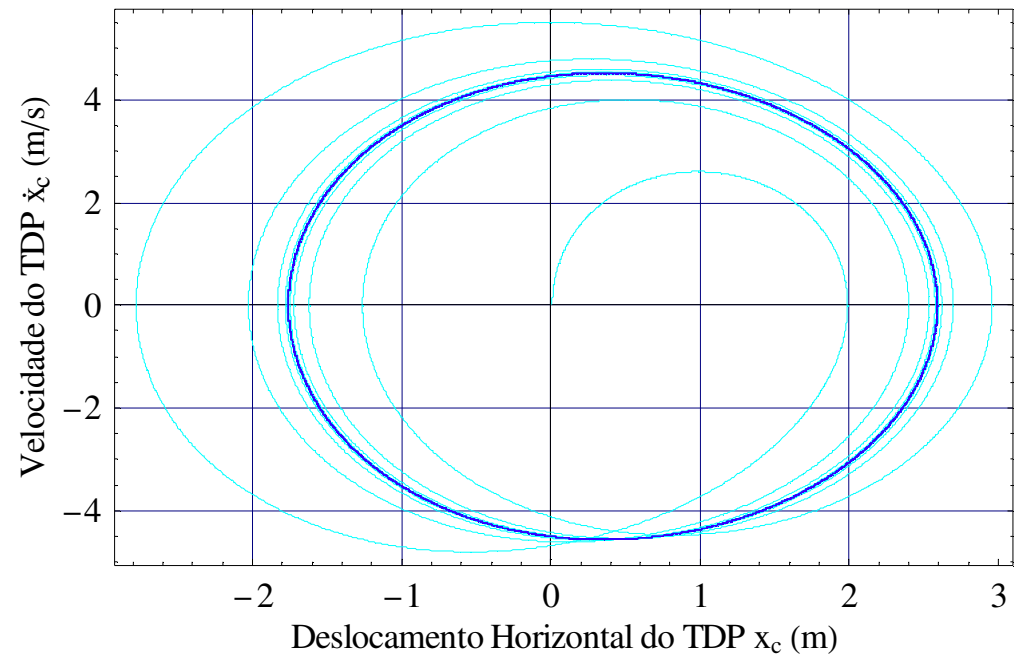

Figura 101: Mapa de fase para $T_{0, T D P=50 \mathrm{kN} \text { e }} \mathrm{T}_{\text {MOD. ORDEM REDUZIDA }}=6.0 \mathrm{~s}$

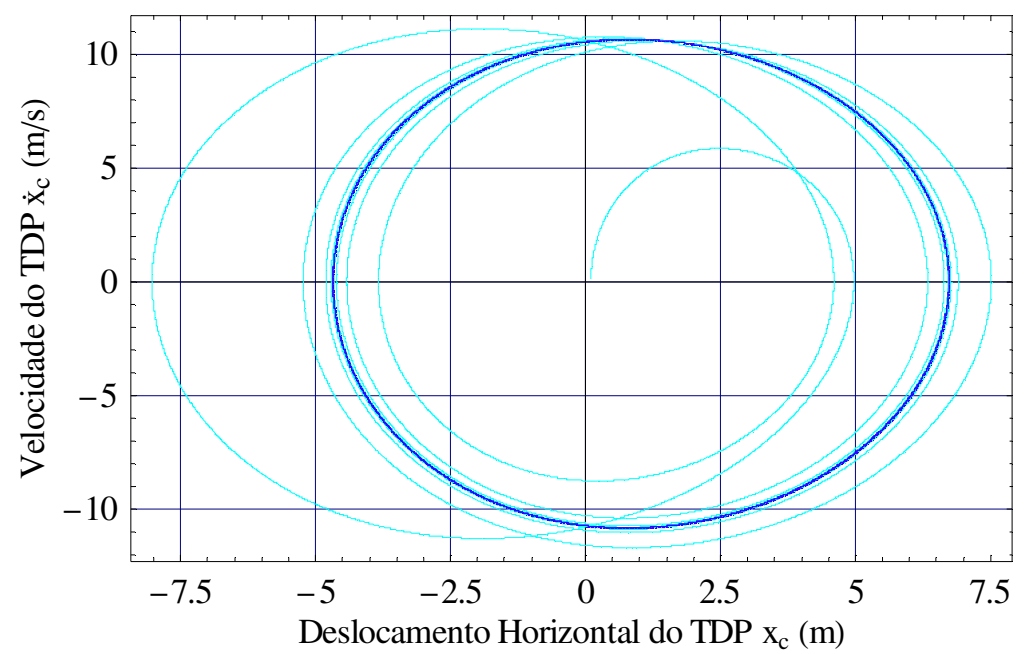

Figura 102: Mapa de fase para $T_{0, T D P}=60 \mathrm{kN}$ e $T_{\text {MOD. ORDEM REDUZIDA }}=6.6 \mathrm{~s}$ 


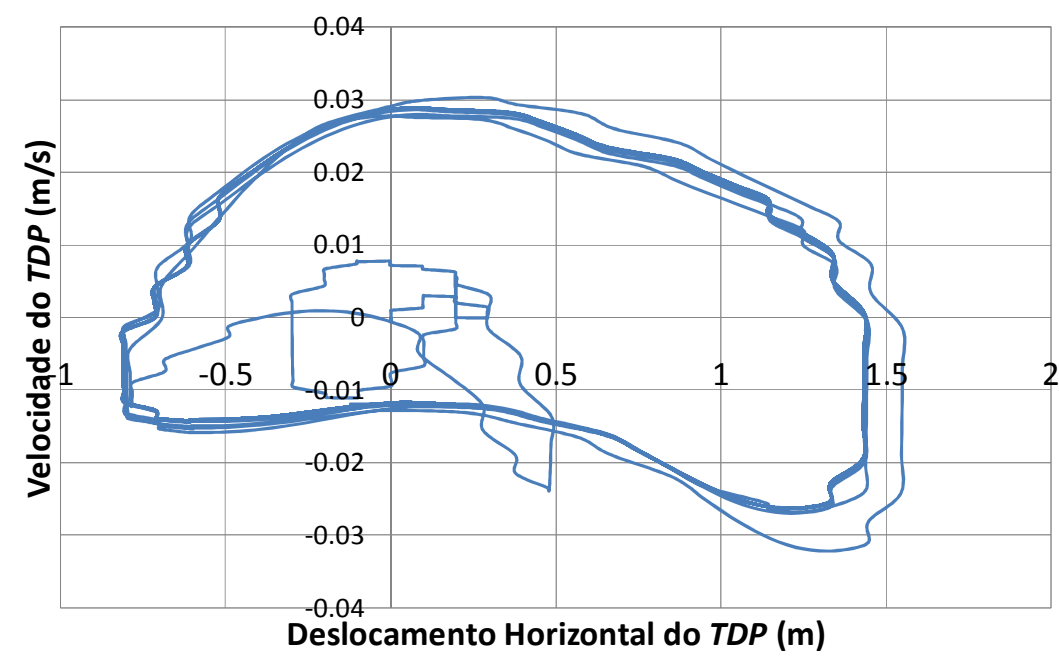

Figura 103: Mapa de fase obtido com o programa Orcaflex para $T_{0, T D P}=35 \mathrm{kN}$ e $T_{\text {TREM DE ONDAS }}=6.2 \mathrm{~s}$

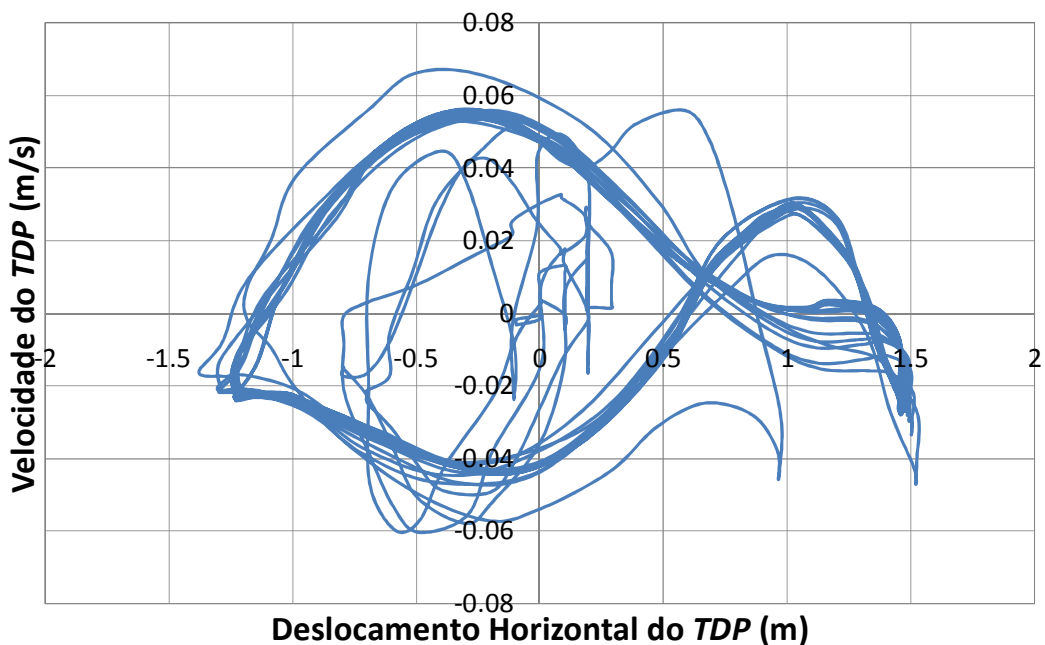

Figura 104: Mapa de fase obtido com o programa Orcaflex para $\mathrm{T}_{0, T D P=50 \mathrm{kN} \text { e }} \mathrm{T}_{\mathrm{TREM} \text { DE }}$ ONDAS$=3.0 \mathrm{~s}$

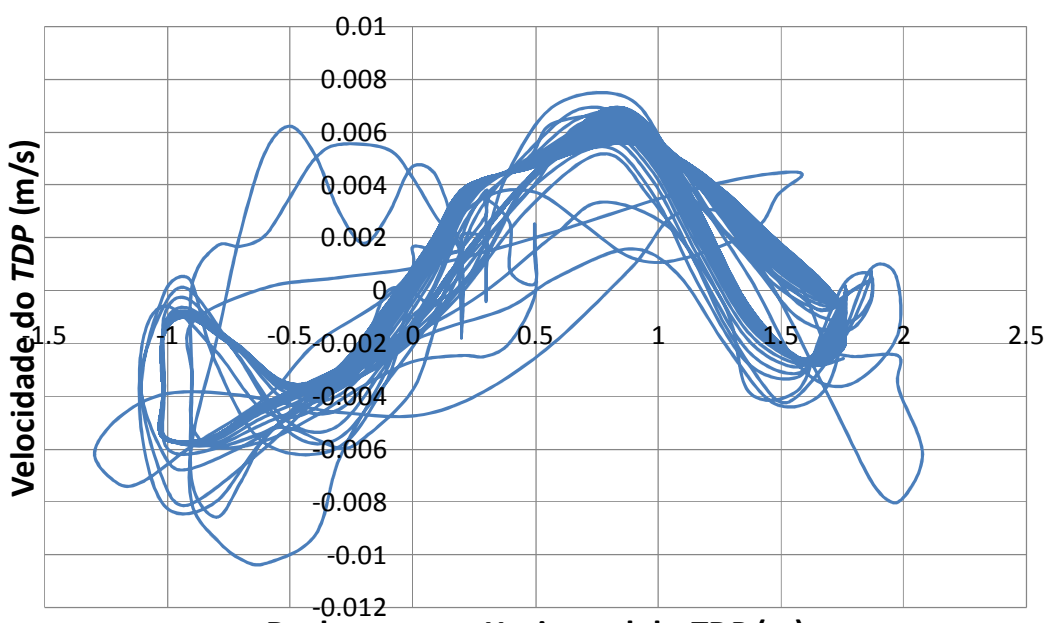

Deslocamento Horizontal do TDP $(\mathrm{m})$

Figura 105: Mapa de fase obtido com o programa Orcaflex para $\mathrm{T}_{0, T D P=60 \mathrm{kN} \text { e }} \mathrm{T}_{\mathrm{TREM} \text { DE }}$ ONDAS=3.3s 
Os resultados dos processamentos segundo o modelo aqui proposto podem ser resumidos na tabela abaixo:

\begin{tabular}{|c||c|c|c|}
\hline Caso & $\mathbf{1}$ & $\mathbf{2}$ & $\mathbf{3}$ \\
\hline \hline $\boldsymbol{X}_{\text {TDP }}(\boldsymbol{m})$ & 17.0 & 4.0 & 11.0 \\
\hline $\boldsymbol{v}_{\text {TDP }}(\mathbf{m} / \mathbf{s})$ & 16.0 & 8.5 & 22.0 \\
\hline $\boldsymbol{T}_{\text {MOD. ORDEM REDUZIDA }}(\boldsymbol{s})$ & 12.4 & 6.0 & 6.6 \\
\hline
\end{tabular}

Tabela 14: Resultados do processamento com excitação paramétrica

Para os mesmos valores de entrada da Tab. 13, os resultados obtidos após processamento com programa Orcaflex podem ser resumidos na Tab. 15:

\begin{tabular}{|c||c|c|c|}
\hline Caso & $\mathbf{1}$ & $\mathbf{2}$ & $\mathbf{3}$ \\
\hline \hline $\boldsymbol{X}_{\text {TDP }}(\boldsymbol{m})$ & 2.3 & 3.0 & 2,5 \\
\hline $\boldsymbol{v}_{T D P}(\boldsymbol{m} / \mathbf{s})$ & 0.06 & 0.12 & 0,06 \\
\hline
\end{tabular}

Tabela 15: Resultados do processamento com Ocaflex com excitação paramétrica

Nota-se, a partir dos resultados expressos nas Tab. 14 e 15, que a ordem de grandeza para deslocamentos é próxima, ainda que a mesma constatação não possa ser verificada em relação à velocidade. A assimetria verificada na velocidade dos mapas de fase após processamento no programa Orcaflex se deve provavelmente à modelagem do amortecimento não linear, como do tipo Morrison, proporcional ao quadrado da velocidade, utilizado na rotina de cálculo do programa.

Supõe-se que os valores extremamente baixos verificados no eixo das velocidades decorram dos efeitos do atrito ao longo da linha na dinâmica longitudinal, que não foi considerada no presente estudo.

Recorde-se que no processamento segundo o modelo aqui apresentado, a rigidez do solo foi de $\varphi=10^{4} \mathrm{~N} / \mathrm{m}^{3}$ e $\varphi=10^{5} \mathrm{~N} / \mathrm{m}^{3}$, valores baixos, o que pode estar associado às diferenças em relação aos processamentos através do Orcaflex. 
A mesma boa aderência que se obteve com o programa Orcaflex, para os casos com ressonância paramétrica, não pôde ser verificada no programa Poliflex 3D. Vale lembrar que o programa Poliflex 3D utiliza a técnica da camada limite, o que faz com que a abordagem difira do modelo proposto no presente texto. 


\section{Conclusão e considerações finais}

Recorde-se inicialmente, como descrito no capítulo 2, de se tratar de um problema de condições de contorno móveis para o qual se utilizou teoria elementar de barras, porém com não linearidades inerentes ao contato unilateral elástico, e que, através de uma transformação de coordenadas, passou a um problema com condições de contorno fixas. Isso fez com que um problema com formulação segmentadamente linear em cada domínio passasse a um problema com não linearidades, com fortes implicações na abordagem analítica.

No capítulo 2 verificou-se, também, que o número de modos de vibração para a viga esbelta semi-infinita é finito, e não infinito, para valores de $c_{0}$ menor que 25, aproximadamente. Para valores maiores, o número de modos de vibração tende a infinito.

No capítulo 3 foi possível formular o problema com flexão composta, introduzindo os esforços de tração. Tal modificação mostrou as limitações da abordagem analítica, que foram contornadas projetando a equação dos deslocamentos dinâmicos com efeito da tração segundo os modos obtidos na seção 2.2, para o modelo com flexão simples, por meio do método de Galerkin.

Recorde-se ter sido adotado amortecimento linear equivalente para representar tanto o amortecimento fluido-dinâmico como o estrutural. Dado que o amortecimento foi adotado de forma "ad hoc", poder-se-ia considerar um amortecimento para cada caso, isto é, um que representasse o fluido-dinâmico, quadrático na velocidade, e outro que representasse 0 estrutural. Tal procedimento não foi adotado no presente trabalho, em função da maior simplicidade de um amortecimento elástico linear equivalente.

Por fim, no capítulo 4, pode-se discutir um estudo de caso, inicialmente com uma análise paramétrica mais ampla, na seção 4.1, para depois aplicar o modelo às condições típicas de riser em catenária e estados de mar, na seção 4.2, focando na possibilidade de ocorrência de ressonância paramétrica. $\mathrm{Na}$ 
seção 4.3 foi possível recuperar os esforços de flexão, força normal e força cortante a partir da solução completa, o que permitiu avaliar a variação das tensões normais e cisalhantes ao longo do tempo, esforços estes muito relevantes na análise da fadiga. Na seção 4.4 discutiu-se a utilização do modelo para casos em que não ocorre ressonância paramétrica, comparando os resultados obtidos com aqueles do programa Poliflex 3D, e na seção 4.5 casos em que ocorre ressonância paramétrica, comparando os resultados com aqueles do programa Orcaflex, em condições semelhantes.

O presente estudo mostra que o modelo de viga esbelta com contato unilateral em meio elástico sujeita a flexão composta tem potencial para ser usado na análise de risers de aço em catenária (SCR).

Em todos os casos estudados verificaram-se maiores deslocamentos naqueles em que a tração estática no ponto "O" é mais baixa. Isso se deve ao fato de que maior tração estática no ponto "O”, por sua vez, gera maior rigidez flexional na linha, e portanto o sistema de uma maneira geral tende a ter amplitudes de deslocamento na resposta dinâmica mais baixas.

Aparentemente, o modelo não linear possui diferentes atratores periódicos para certos valores de tração estática e dinâmica. Em boa parte dos processamentos houve majoração dos deslocamentos frente às condições iniciais fornecidas. Neste contexto, mais uma vez, cita-se o trabalho desenvolvido em Gonçalves et al [18], e a possibilidade de abordagem do problema quanto à integridade de bacias de atração.

Verificou-se, através dos mapas de Poincaré, uma variedade de soluções, que incluem: solução quase periódica, após bifurcação de Hopf da solução harmônica (caso T1), solução com número elevado de períodos (caso T2), e até mesmo solução de múltiplos períodos com algum efeito de transiente e possível proximidade de um regime caótico (caso T10), entre outras soluções com período bem definido. Isto indica um comportamento altamente não-linear e a 
necessidade de se investigar no futuro os diagramas de bifurcação, coexistência de soluções e, neste caso, as bacias de atração deste problema.

Estabeleceu-se a relação entre a tração estática no TDP e as freqüências naturais de vibração do modelo. $O$ aumento da tração estática leva ao aumento da freqüência de vibração, e, portanto, a períodos menores. Isso faz com que para valores de tração estática maiores as condições de ressonância paramétrica praticamente desapareçam, pois o período do trem de ondas deveria ser muito pequeno e constante, portanto fora dos padrões reais observados.

Os valores de deslocamento obtidos na resposta da excitação paramétrica não são desprezíveis: da ordem de $20 \mathrm{~m}$, para taxas de amortecimento linear equivalente $\xi=30 \%$, em regime estacionário. Tais valores mostram que 0 sistema é altamente sensível às condições iniciais, e que, para trações estáticas mais baixas, a probabilidade de ocorrência é ainda maior.

Os valores de $c_{0}$ obtidos e considerados nos estudos de caso, e os seus finitos modos de vibração, estão diretamente associados ao fato que a rigidez flexional do riser é relevante apenas nas proximidades do TDP, e não em toda a sua extensão. Como se sabe, o longo trecho suspenso é fortemente dominado pelo regime de membrana (cabo).

As diferenças quanto aos resultados entre os processamentos do modelo e aqueles utilizando o programa Poliflex 3D, mostram que as não linearidades não são desprezíveis, e não devem ser descartadas "ad limina". A maior evidência são as diferentes respostas geradas para cada caso, onde houve majoração substancial dos deslocamentos e velocidades no modelo em que as não linearidades foram contempladas.

Recorde-se que, de posse dos resultados apresentados, foi possível fazer o caminho inverso das transformações que levaram à adimensionalização do problema, de forma que, a partir de $\delta(z, \tau)$ foi possível obter $u(z, \tau)$, o que nos levou à $v(y, \tau)$, e que por fim nos permitiu obter a relação para o deslocamento 
vertical dimensional $w(x, t)$. A partir destes deslocamentos, pôde-se estabelecer as correlações que nos permitiram avaliar as solicitações de momento fletor, força normal e força cortante, respectivamente, $M(x, t)=-E I w^{\prime \prime}(x, t), \quad T(x, t)=$ $\left[T_{0}+T_{1} \cos (\Omega t)\right] e^{-a x}$ e $V(x, t)=-E I w^{\prime \prime \prime}(x, t)$. Assim, a partir dos resultados apresentados na seção 4.3, pôde-se chegar às solicitações que permitiram obter dados relevantes ao estudo de fadiga da viga ou riser em questão.

Alguns pontos devem ser considerados como limitações do modelo, e podem ser interpretados como oportunidades de melhoria no futuro.

Recorde-se tratar de um modelo complexo, altamente não linear (apesar de ter sido usada a teoria elementar de barras, linearizando-se a curvatura de flexão), mas que teve seus modos de vibração determinados até primeira ordem e, ainda assim, apenas para o caso da flexão simples. Talvez haja possibilidade de desenvolver a metodologia considerando termos de ordem superior na aplicação do método das múltiplas escalas no problema da flexão simples para obtenção dos modos.

Verificou-se também razoável sensibilidade nos resultados dos processamentos feitos, principalmente na seção 4.1., onde se constatou que a taxa de amortecimento, mesmo que pequena, da ordem de 1\%, é capaz de mudar drasticamente o histórico de resposta. A partir de outras condições, vale a consideração de possíveis estudos sobre a integridade de bacias de atração.

Uma observação pode ser feita no que diz respeito à relação entre o comprimento do trecho de viga apoiado $L$ no qual a tração tende a valores baixos, utilizado nos limites de integração dos coeficientes descritos em (101) que fazem parte da rotina que leva ao modelo de ordem reduzida em (111), e a função modal $\zeta(z)$ em (76) e (77). Dado que o produto da função modal com outras funções determina a faixa na qual o modo tem influência e, portanto, na qual a perturbação pode ocorrer, é a função modal quem acaba por governar o comprimento efetivo do modo de vibração. No entanto, os valores de $L$ foram determinados segundo o decaimento da tração a valores baixos, o que não tem 
relação direta com a função modal, e acaba interferindo nos valores das integrais em (101). Uma alternativa é fazer com que o comprimento $L$ considerado seja, de fato, associado à função modal, o que daria maior coerência ao modelo.

As trações estática e dinâmica no TDP, bem como as trações estática e dinâmica no ponto "O", utilizadas na integração do oscilador modal, poderiam ser determinadas de maneira analítica e integradas à rotina de cálculo, conforme Aranha e Pinto [3], de forma a tornar a metodologia independente dos resultados do programa Poliflex 3D ou outro equivalente.

Considera-se, também, a possibilidade de acrescentar à tração imposta no ponto "O" o deslocamento dinâmico, de forma a representar de forma mais fidedigna a realidade física.

É um considerável desafio o desenvolvimento de uma metodologia semelhante para o problema em três dimensões. Neste contexto, cita-se o trabalho desenvolvido em Crespo da Silva e Glynn [13].

Há possibilidade de desenvolvimento de soluções analíticas forçadas, usando o MME, fornecendo subsídios para identificação de eventuais atratores periódicos e discussão de sua estabilidade. Neste contexto, pode-se considerar a extensão do estudo para modelagem visando vários modos não lineares não acoplados e também multimodos não lineares com acoplamento. 


\section{Referências Bibliográficas}

[1] Aranha, J.A.P.; Pinto, M.O.; Leite, A.J.P.; Dynamic tension of cables in a random sea: analytic approximation for the envelope probability density function, Applied Ocean Research, 23, pp. 93101,2001

[2] Aranha, J.A.P.; Pinto, M.O.; Silva, R.M.C.; On the dynamic compression of risers: an analytic expression for the critical load, Applied Ocean Research, 23, pp. 83-91, 2001

[3] Aranha, J.A.P.; Pinto, M.O.; Dynamic tension in risers and mooring lines: an algebraic approximation for harmonic excitation, Applied Ocean Research, 23, pp. 63-81, 2001

[4] Baracho Neto, O.G.P.; Modos normais e multimodos na dinâmica das estruturas de comportamento não linear, Tese de Doutorado, Escola Politécnica da Universidade de São Paulo, São Paulo, 2003

[5] Baracho Neto, O.G.P.; Redução de graus de liberdade: uma proposta para a análise dinâmica de estruturas aporticadas planas de comportamento geometricamente não linear, Dissertação de Mestrado, Escola Politécnica da Universidade de São Paulo, São Paulo, 1998

[6] Blevins, R.D.; Flow-induced vibration, Second Edition, Krieger Publishing Company, 2001

[7] Chatjigeorgiou, I.K.; $A$ finite differences formulation for the linear and nonlinear dynamics of $2 D$ catenary risers, Ocean Engineering, 35, pp. 616-636, 2008

[8] Chatjigeorgiou, I.K.; Application of the WKB method to catenary-shaped slender structures, Mathematical and Computer Modeling, 48, pp. 249-257, 2008

[9] Chatjigeorgiou, I.K.; Solution of the boundary layer problems for calculating the natural modes of riser-type slender structures, Journal of Offshore Mechanics and Arctic Engineering, 130, 2008

[10] Chatjigeorgiou, I.K.; Mavrakos, S.A.; Bounded and unbounded coupled transverse response of parametrically excited vertical marine risers and tensioned cable legs for marine applications, Applied Ocean Research, 24, pp. 341-354, 2002

[11] Clough, R.W.; Penzien, J.; Dynamics of structures, McGraw-Hill, 1975 
[12] Crespo da Silva, M.R.M.; Glynn, C.C.; Non-linear non-planar resonant oscillations in fixed-free beams with support asymmetry, International Journal of Solids and Structures, 15, pp. 209-219, 1979

[13] Crespo da Silva, M.R.M.; Glynn, C.C.; Out-of-plane vibrations of a beam including non-linear inertia and non-linear curvature effects, International Journal of Solids and Structures, 13, pp. 261271,1979

[14] Cunha, L.D.; Vibração induzida por vórtices: análise crítica de modelos fenomenológicos, Tese de Mestrado, Escola Politécnica da Universidade de São Paulo, São Paulo, 2005

[15] Demeio, L.; Lenci, S.; Forced nonlinear oscillations of semi-infinite cables and beams resting on a unilateral elastic substrate, Nonlinear Dyn, 49, pp. 203-215, 2007

[16] Demeio, L.; Lenci, S.; Second order solutions for the dynamics of a semi-infinite cable on a unilateral substrate, Journal of Sound and Vibration, 315, pp. 414-432, 2008

[17] Fujarra, A.; Estudos experimentais e analíticos das vibrações induzidas pela emissão de vórtices em cilindros flexíveis e rígidos, Tese de Doutorado, Escola Politécnica da Universidade de São Paulo, São Paulo, 2002

[18] Gonçalves, P.B.; Silva, F.M.A.; Rega, G.; Lenci, S.; Global dynamics and integrity of a two-dof model of a parametrically excited cylindrical shell, Nonlinear Dyn, pp. 61-82, 2011

[19] Josefsson, M.; Dalton, C.; An analytical/computational approach in assessing vortex-induced vibration of a variable tension riser, Journal of Offshore Mechanics and Arctic Engineering, 132, 2010

[20] Martins, C.A.; Takafugi, F.C.M.; Tanaka, R.L.; Silveira, L.M.Y.; Neto, A.G.; Nascimento Jr, K.C.; Oliveira, L.H.S.; Nunes, R.P.; Poliflex 3D, v. 2.0.1, 2008

[21] Mazzilli, C.E.N.; Lenci, S.; Vibrazioni libere non-lineari nei dintorni della configurazione di equilibrio static di una trave con contatto elastic unilaterale, comunicação pessoal dos autores

[22] Mazzilli, C.E.N.; Lenci, S.; Normal vibration modes of a slender beam on elastic foundation with unilateral contact, ICTAM, XXII, pp. 25-29, 2008

[23] Mazzilli, C.E.N.; Sanches, C.T.; Baracho Neto, O.G.P.; Wiercigroch, M.; Keber, M.; Non-linear modal analysis for beams subjected to axial loads: analytical and finite-element solutions, International Journal of Non-Linear Mechanics, doi: 10.1016, 2008 
[24] Mazzilli, C.E.N.; Soares, M.E.S.; Baracho Neto, O.G.P.; Non-linear normal modes of a simply supported beam: continuous system and finite-element models, Computers and Structures, 82, pp. 2683-2691, 2004

[25] Mazzilli, C.E.N.; Wiercigroch, M.; Effect of linearly varying normal force upon the nonlinear modal analysis of slender beams, Enoc, 4, 2008

[26] Meneghini, J.R.; Projetos de pesquisa no tópico geração e desprendimento de vórtices no escoamento ao redor de cilindros, Livre Docência, Escola Politécnica da Universidade de São Paulo, São Paulo, 2002

[27] Nayfeh, A.H.; Mook, D.T.; Nonlinear oscillations, Wiley, 1979

[28] Pesce, C.P.; Martins, C.A.; Silveira, L.M.Y.; Riser-soil interaction: local dynamics at TDP and a discussion on the eigenvalue and the VIV problems, Journal of Offshore Mechanics and Arctic Engineering, 128, 2006

[29] Pesce, C.P.; Mecânica de cabos e tubos submersos lançados em "catenária": uma abordagem analítica e experimental, Livre Docência, Escola Politécnica da Universidade de São Paulo, São Paulo, 1997

[30] Ramos, R.Jr.; Pesce, C.P.; A stability analysis of risers subjected to dynamic compression coupled with twisting, Journal of Offshore Mechanics and Arctic Engineering, 125, 2003

[31] Sanches, C.T.; Modos não lineares de vibração e controle ativo de risers, Tese de Doutorado, Escola Politécnica da Universidade de São Paulo, São Paulo, 2009

[32] Sanches, C.T.; Mazzilli, C.E.N.; Cunha, L.D.; Pesce, C.P.; Non-linear analysis applied to riser dynamics, ISOPE, JSC215, 2007

[33] Scheidl, R.; Troger, H.; Zeman, K.; Coupled flutter and divergence bifurcation of a double pendulum, International Journal of Non-Linear Mechanics, 19, pp. 163-176, 1983

[34] Silva, A.P.; Nogueira, L.; Relatórios de curso da disciplina Projeto do Navio III, COPPE-UFRJ, 2010

[35] Silveira, L.M.Y.; Modelo hidro-elástico para simular as vibrações induzidas por vórtices em cabos submersos, Tese de Doutorado, Escola Politécnica da Universidade de São Paulo, São Paulo, 2009 
[36] Simos, A.N.; Fujarra, A.L.C.; Dynamic compression of rigid and flexible risers: experimental and numerical results, Journal of Offshore Mechanics and Arctic Engineering, 128, 2006

[37] Song, R.; Stanton, P.; Advances in deepwater steel catenary riser technology state of the art: part I - design, International Conference on Offshore Mechanics and Arctic Engineering, 26 ${ }^{\text {th }}, 2007$

[38] Sorokin, S.V.; Rega, G.; On modeling and linear vibrations of arbitrarily sagged inclined cables in a quiescent viscous fluid, Journal of Fluids and Structures, 23, pp. 1077-1092, 2007

[39] Takafuji, F.C.M.; Dinâmica tridimensional de risers, Tese de Doutorado, Escola Politécnica da Universidade de São Paulo, São Paulo, 2010

[40] Yamamoto, C.T.; Meneghini, J.R.; Saltara, F.; Fregonesi, R.A.; Ferrari Jr., J.A.; Numerical simulations of vortex-induced vibration on flexible cylinders, Journal of Fluids and Structures, 19, pp. 467-489, 2004 


\section{Anexos}

\subsection{Transformação de coordenadas para obtenção do problema com condições de contorno fixas}

A partir da equação original normalizada:

$\frac{1}{4} \frac{\partial^{4} v}{\partial y^{4}} v+\frac{\partial^{2} v}{\partial \tau^{2}}+H v+1=0$,

e fazendo uso da transformação de variáveis:

$z=\frac{y}{c(\tau)}-1 \Rightarrow y=(z+1) c(\tau)$,

obtemos as derivadas temporais e espaciais para as expressões:

$\frac{d z}{d \tau}=-\frac{\dot{c}}{c^{2}} y=-\frac{\dot{c}}{c^{2}}(z+1) c=-\frac{\dot{c}}{c}(z+1)$,

$\frac{\partial}{\partial y}=\frac{\partial}{\partial z} \cdot\left(\frac{d z}{d y}\right)=\frac{1}{c} \cdot \frac{\partial}{\partial z}$,

$\frac{\partial^{4}}{\partial y^{4}}=\frac{1}{c^{4}} \cdot \frac{\partial^{4}}{\partial z^{4}}$.

Fazendo uso da relação entre as variáveis:

$v(y, \tau)=u(z, \tau)$

obtemos os valores para:

$\frac{\partial^{4}}{\partial y^{4}} v=\frac{1}{c^{4}} \frac{\partial^{4}}{\partial z^{4}} u=\frac{1}{c^{4}} u^{I V}$, 
$\frac{\partial}{\partial \tau} v=\frac{\partial}{\partial \tau} u=\frac{\partial u}{\partial z} \cdot \frac{d z}{d \tau}+\frac{\partial u}{\partial \tau}=-\frac{u^{\prime} \dot{c}}{c}(z+1)+\dot{u}$

$$
\begin{aligned}
\frac{\partial^{2}}{\partial \tau^{2}}=\frac{\partial}{\partial \tau}\left[-\frac{u^{\prime} \dot{c}}{c}\right. & (z+1)+\dot{u}] \\
& =-\frac{u^{\prime} \dot{c}}{c}(z+1)+u^{\prime \prime} \frac{\dot{\mathrm{c}}^{2}}{\mathrm{c}^{2}}(1+z)^{2}-u^{\prime}(z+1) \frac{\ddot{c} \mathrm{c}-\dot{\mathrm{c}}^{2}}{\mathrm{c}^{2}}+u^{\prime} \frac{\dot{\mathrm{c}}^{2}}{\mathrm{c}^{2}}(z+1) \\
& -2 \dot{u}^{\prime} \frac{\dot{\mathrm{c}}}{\mathrm{c}}(\mathrm{z}+1)=-\dot{u}^{\prime} \frac{\dot{\mathrm{c}}}{\mathrm{c}}(z+1)(1+\ddot{u})+u^{\prime \prime} \frac{\dot{c}^{2}}{c^{2}}(1+z)^{2}+u^{\prime}(z+1) \frac{2 \dot{c}^{2}-\ddot{c} c}{c^{2}} \\
& +u^{\prime} \frac{\dot{c}^{2}}{c^{2}}(z+1)+\ddot{u}-\dot{u}^{\prime} \frac{\dot{c}}{c}(z+1) .
\end{aligned}
$$

Substituindo os valores encontrados, chega-se a:

$$
\begin{gathered}
\frac{1}{4 c^{4}} u^{I V}-2 \dot{u}^{\prime} \frac{\dot{\mathrm{c}}}{\mathrm{c}}(\mathrm{z}+1)+u^{\prime \prime} \frac{\dot{c}^{2}}{c^{2}}(1+z)^{2}+u^{\prime}(z+1) \frac{2 \dot{c}^{2}-\ddot{c} c}{c^{2}}+u^{\prime} \frac{\dot{c}^{2}}{c^{2}}(z+1)+\ddot{u}+H u \\
+1=0
\end{gathered}
$$

e multiplicando a equação acima por $c^{4}$, chega-se à expressão final utilizada no desenvolvimento do texto:

$$
\begin{gathered}
\frac{1}{4} u^{I V}-2 \dot{u}^{\prime} \dot{c}(\mathrm{z}+1) c^{3}+u^{\prime \prime} \dot{c}^{2}(1+z)^{2} c^{2}+u^{\prime}(z+1)\left(2 \dot{c}^{2}-\ddot{c} c\right) c^{2}+c^{4} \ddot{u}+H c^{4} u+c^{4} \\
=0
\end{gathered}
$$




\subsection{Comparação da configuração estática}

A figura abaixo ilustra, como exemplo, a comparação entre a configuração estática fornecida pelo programa Poliflex 3D e a solução estática aproximada pelo modelo, através de um polinômio de quarto grau:

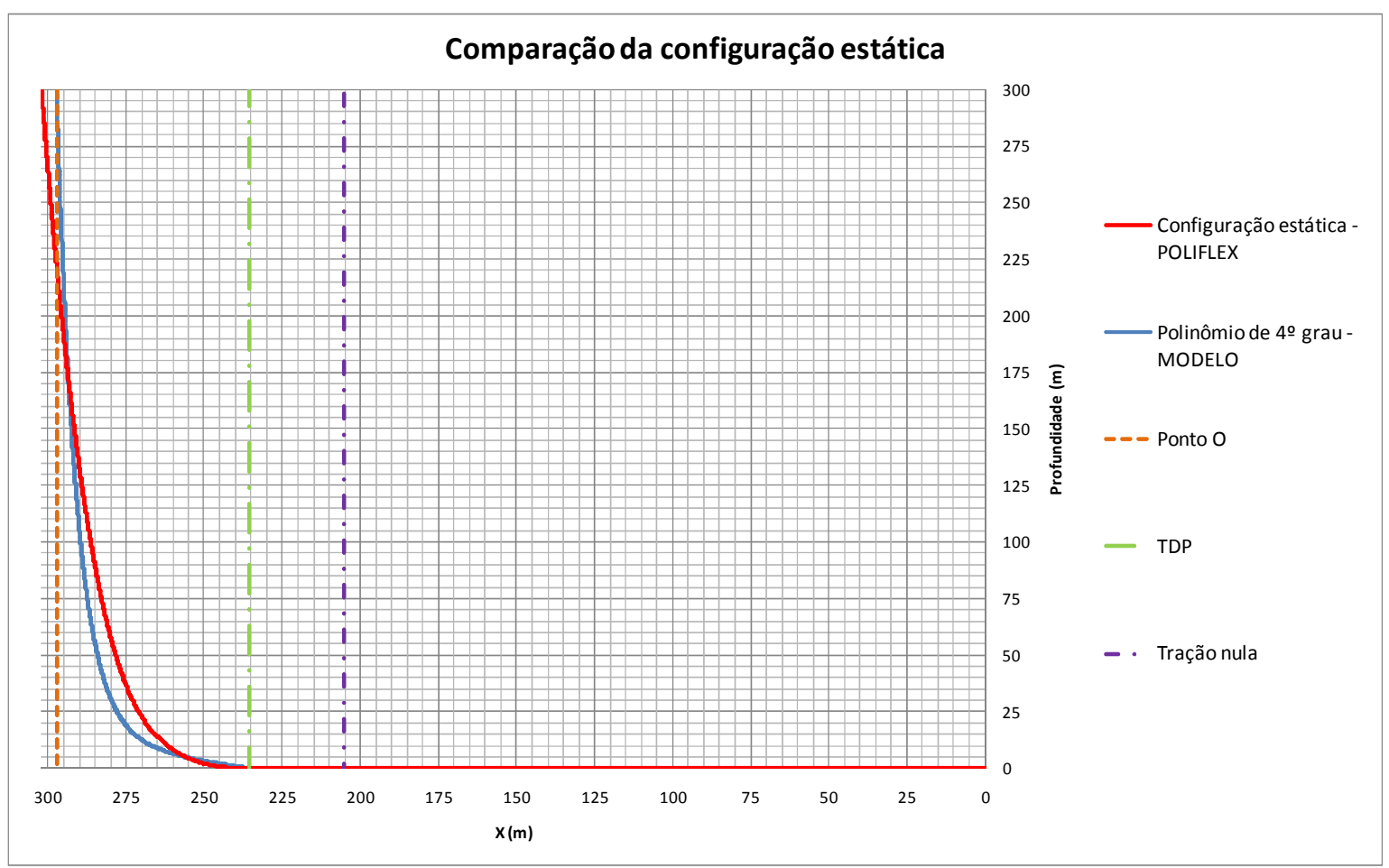

Figura 106: Comparação da configuração estática

Recorde-se que, no desenvolvimento do texto, não foi adotada a formulação de catenária para caracterizar o deslocamento estático da linha, mas sim a integração das equações diferenciais (12) e (90), o que resulta nos polinômios (15) e (94). Por este motivo, compara-se para um dos casos estudados, a resposta fornecida pelo programa Poliflex 3D, que usa a formulação de cabo em catenária na solução estática do problema, como medida de comparação.

No entanto, mesmo que o modelo proposto tenha partido da formulação com curvatura linearizada, foi levado em consideração o efeito de flexão, algo que não acontece na rotina de cálculo do programa Poliflex $3 \mathrm{D}$, mesmo que 0 programa respeite a não linearidade do cabo. 


\subsection{Parâmetro $\theta$ na consideração do efeito da tração}

Recuperando as expressões (91) e (92), que consideram o efeito da tração no problema:

$\gamma(z, \tau)=\Gamma e^{-\theta(1+z)}=\left[\Gamma_{0}+\Gamma_{1} \cos (\Omega \tau)\right] e^{-\theta(1+z)}$,

o valor de $\theta$ é determinado considerando o decaimento da força normal, a partir do TDP, até um valor em que esta represente $1 \%$ da força no TDP. No gráfico abaixo os dois modelos utilizados para o cálculo:
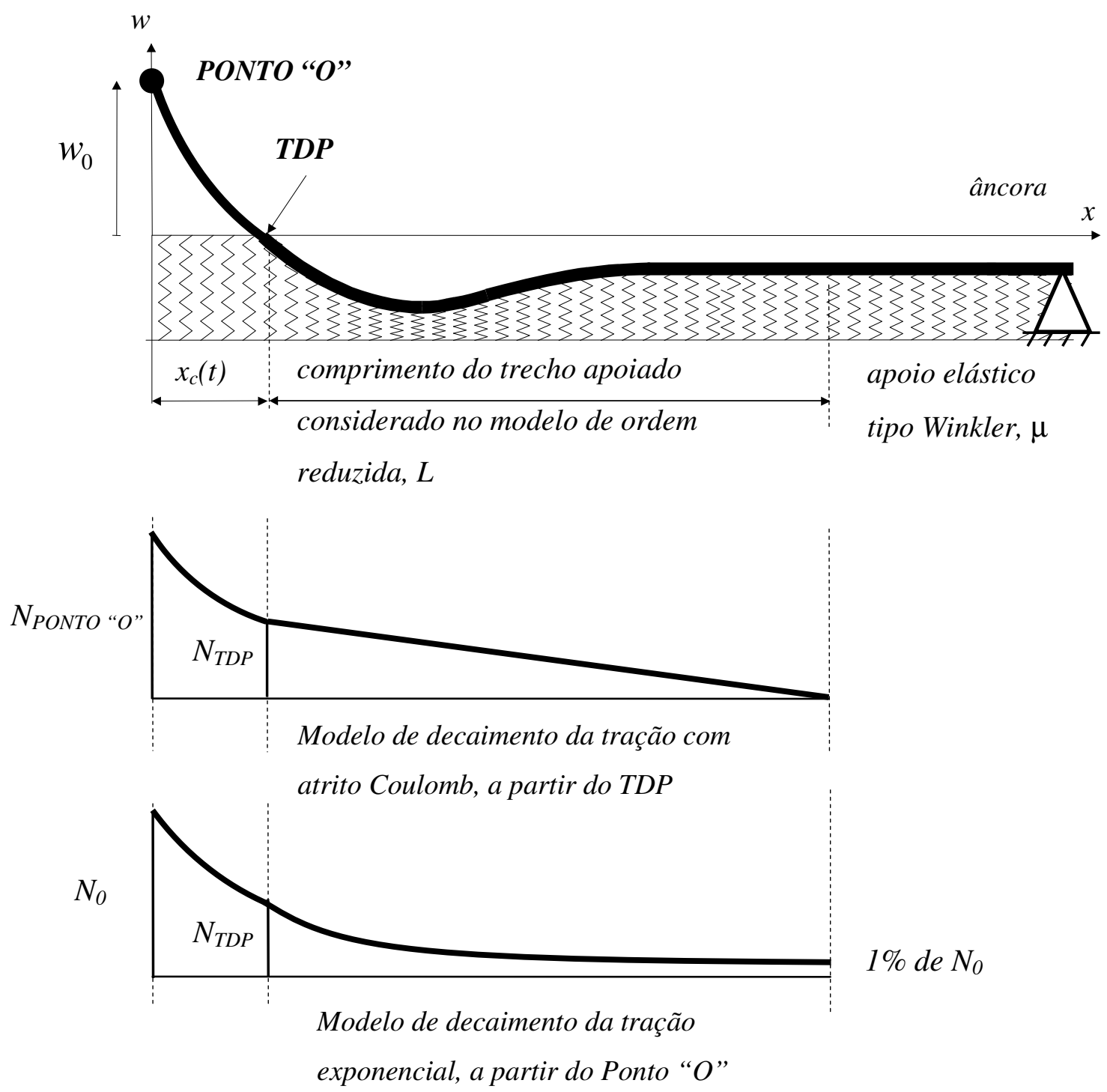

Figura 107: Modelo de decaimento da tração ao longo da linha 
A figura abaixo ilustra, a partir de dados do programa Poliflex 3D, como exemplo, a comparação entre os dois modelos:

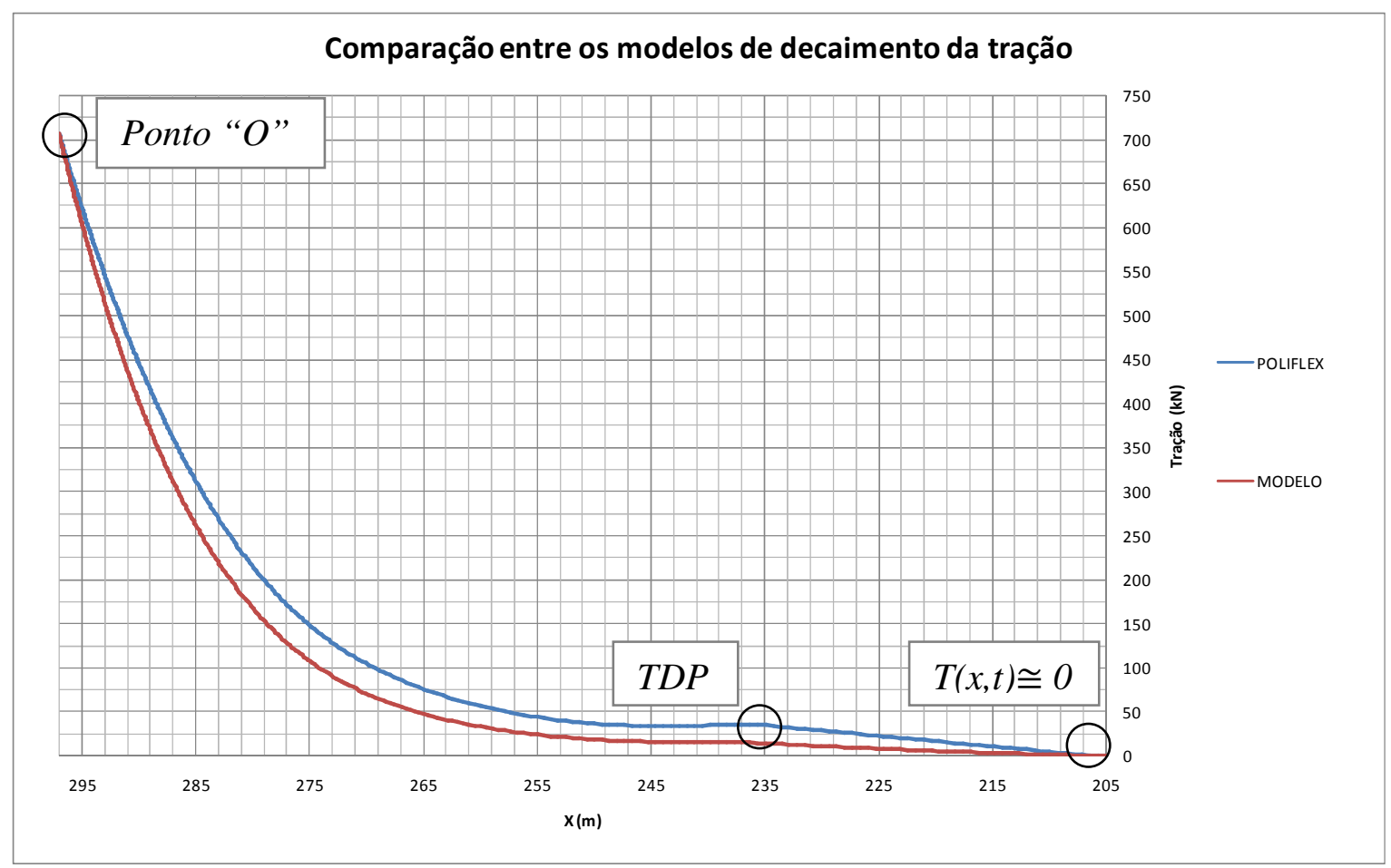

Figura 108: Comparação entre os modelos de decaimento da tração

Partindo da premissa que o decaimento linear se dá através da relação:

$L_{k}=\frac{N_{0}}{p \cdot k}$

sendo $N_{0}$ a força de tração no TDP, $p$ o peso submerso do cabo e $k$ o coeficiente de atrito do solo, faz-se a seguinte associação para o decaimento exponencial:

$N(x)=N_{0} e^{-a x}$,

tal que:

$N\left(L_{k}\right)=0,01 N_{0}=N_{0} e^{-a L_{k}}$. 
Decorre portanto:

$a=-\frac{\ln (0,01)}{L_{k}}$,

sendo $\theta$ a forma de $a$ adimensionalizado:

$\theta=a \frac{c_{0}}{\alpha}$. 


\subsection{Tabela dos resultados obtidos de processamentos feitos no programa Poliflex 3D, v. 2.0.1}

Os resultados abaixo foram extraídos de processamento no programa Poliflex 3D, v. 2.0.1., Martins et al [20]. De posse destes dados pode-se embasar as simulações numéricas feitas, principalmente por avaliar a ordem de grandeza para a tração estática e dinâmica no TDP em função de profundidade e comprimento do cabo.

\begin{tabular}{|c|c|c|c|c|c|}
\hline Modelo & \begin{tabular}{|c} 
Profundidade \\
$Z(m)$
\end{tabular} & $\begin{array}{c}\text { Comprimento } \\
L(m)\end{array}$ & $\begin{array}{l}\text { Tração estática } \\
\text { no TDP } T_{0}(k N)\end{array}$ & $\begin{array}{c}\text { Amplitude da tração } \\
\text { dinâmica no TDP } 2 T_{1}(k N)\end{array}$ & $T_{1} / T_{0}$ \\
\hline 1 & 500 & 900 & 493,8 & 29,5 & $3,0 \%$ \\
\hline 2 & 500 & 900 & 268,1 & 14,5 & $2,7 \%$ \\
\hline 3 & 500 & 900 & 180,7 & 9,8 & $2,7 \%$ \\
\hline 4 & 500 & 900 & 151,7 & 8,1 & $2,7 \%$ \\
\hline 5 & 300 & 550 & 530,4 & 20,8 & $2,0 \%$ \\
\hline 6 & 300 & 550 & 71,0 & 7,7 & $5,4 \%$ \\
\hline 7 & 300 & 550 & 60,0 & 7,2 & $6,0 \%$ \\
\hline 8 & 300 & 550 & 70,3 & 7,7 & $5,4 \%$ \\
\hline 9 & 300 & 550 & 58,3 & 6,8 & $5,9 \%$ \\
\hline 10 & 500 & 900 & 134,4 & 7,4 & $2,8 \%$ \\
\hline 11 & 500 & 900 & 127,3 & 7,2 & $2,8 \%$ \\
\hline 12 & 500 & 900 & 125,0 & 7,0 & $2,8 \%$ \\
\hline 13 & 500 & 900 & 123,0 & 6,8 & $2,8 \%$ \\
\hline 14 & 200 & 360 & 75,0 & 12,1 & $8,1 \%$ \\
\hline 15 & 200 & 360 & 66,9 & 11,5 & $8,6 \%$ \\
\hline 16 & 300 & 560 & 35,2 & 7,9 & $11,3 \%$ \\
\hline 17 & 500 & 900 & 125,0 & 10,3 & $4,1 \%$ \\
\hline 18 & 500 & 940 & 49,4 & 6,0 & $6,1 \%$ \\
\hline 19 & 200 & 360 & 66,9 & 25,1 & $18,8 \%$ \\
\hline 20 & 200 & 360 & 60,4 & 25,1 & $20,8 \%$ \\
\hline
\end{tabular}

Tabela 16: Resultados de processamentos com programa Poliflex 3D 
7.5. Tabela com levantamento de período de trem de ondas e altura correspondente na Bacia de Campos

\begin{tabular}{|c|c|c|c|c|c|c|c|c|c|}
\hline \multirow{2}{*}{ Altura Hs (m) } & \multicolumn{8}{|c|}{ Período T (s) } & \multirow{2}{*}{ Total } \\
\hline & 3 a 5 & 5 a 7 & 7 a 9 & 9 a 11 & 11 a 13 & 13 a 15 & 15 a 17 & 17 a 19 & \\
\hline 0,0 a 0,5 & 219 & 48 & 20 & 107 & 176 & 205 & 84 & 13 & 872 \\
\hline 0,5 a 1,0 & 1.518 & 1.040 & 373 & 411 & 522 & 212 & 66 & 1 & 4.143 \\
\hline 1,0 a 1,5 & 461 & 1.722 & 1.049 & 550 & 419 & 87 & 15 & 1 & 4.304 \\
\hline 1,5 a 2,0 & 13 & 870 & 787 & 459 & 314 & 91 & 8 & 1 & 2.543 \\
\hline 2,0 a 2,5 & 0 & 175 & 428 & 269 & 267 & 69 & 8 & 0 & 1.216 \\
\hline 2,5 a 3,0 & 0 & 16 & 144 & 137 & 139 & 52 & 12 & 0 & 500 \\
\hline 3,0 a 3,5 & 0 & 3 & 43 & 44 & 77 & 18 & 7 & 0 & 192 \\
\hline 3,5 a 4,0 & 0 & 0 & 3 & 37 & 35 & 22 & 5 & 1 & 103 \\
\hline 4,0 a 4,5 & 0 & 0 & 1 & 16 & 19 & 20 & 3 & 0 & 59 \\
\hline 4,5 a 5,0 & 0 & 0 & 0 & 3 & 4 & 9 & 2 & 0 & 18 \\
\hline 5,0 a 5,5 & 0 & 0 & 0 & 1 & 3 & 4 & 4 & 0 & 12 \\
\hline 5,5 a 6,0 & 0 & 0 & 0 & 0 & 1 & 0 & 0 & 0 & 1 \\
\hline 6,0 a 6,5 & 0 & 0 & 0 & 0 & 0 & 1 & 0 & 0 & 1 \\
\hline 6,5 a 7,0 & 0 & 0 & 0 & 0 & 0 & 1 & 0 & 0 & 1 \\
\hline Total & 2.211 & 3.874 & 2.848 & 2.034 & 1.976 & 791 & 214 & 17 & 13.965 \\
\hline
\end{tabular}

Tabela 17: Tabela resumo com período e altura do trem de ondas na Bacia de Campos, de acordo com levantamento em Silva e Nogueira [34] 